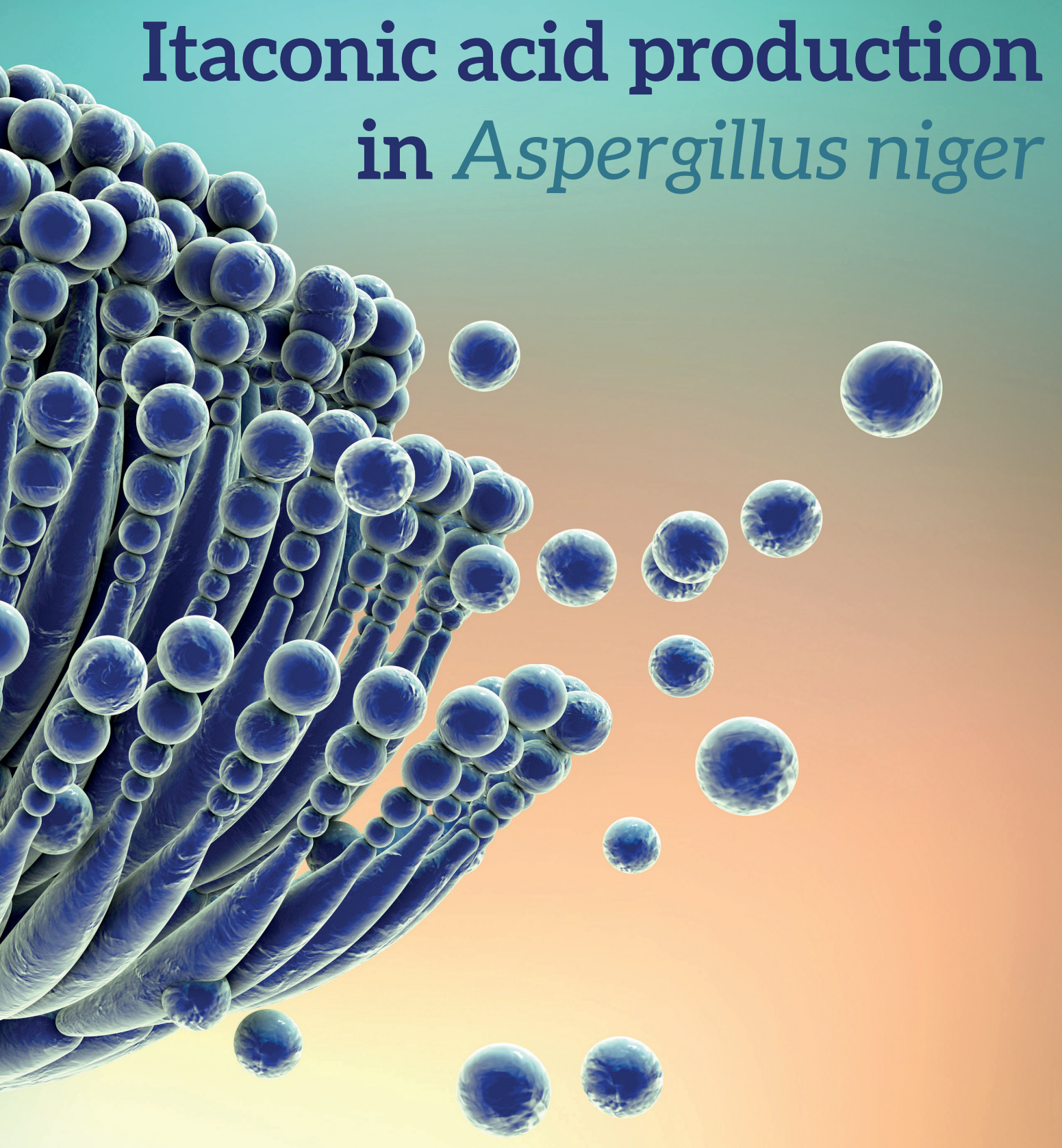

Laura van der Straat 


\section{Itaconic acid production}

\section{in Aspergillus niger}

Laura van der Straat 


\section{Thesis committee}

\section{Promotors}

Prof. Dr V.A.P. Martins Dos Santos

Professor of Systems \& Synthetic Biology

Wageningen University \& Research

Prof. Dr W.J.H. van Berkel

Personal Chair at the Laboratory of Biochemistry

Wageningen University \& Research

\section{Co-promotor}

Dr L.H. de Graaff †

Associate Professor, Systems \& Synthetic Biology

Wageningen University \& Research

\section{Other members}

Prof. Dr J. van der Oost, Wageningen University \& Research

Prof. Dr P.J. Punt, Leiden University

Dr R.P. de Vries, CBS-KNAW Fungal Biodiversity Centre, Utrecht

Dr N. van Peij, DSM Delft

This research was conducted under the auspices of the Graduate School VLAG (Advanced studies in Food Technology, Agrobiotechnology, Nutrition and Health Sciences) 


\title{
Itaconic acid production in Aspergillus niger
}

\author{
Laura van der Straat
}

\section{Thesis}

submitted in fulfilment of the requirements for the degree of doctor at Wageningen University

by the authority of the Rector Magnificus,

Prof. Dr A.P.J. Mol,

in the presence of the

Thesis Committee appointed by the Academic Board

to be defended in public

on Friday 7 December 2018

at 1.30 p.m. in the Aula. 
Laura van der Straat

Itaconic acid production in Aspergillus niger, 156 pages.

PhD thesis, Wageningen University, Wageningen, NL (2018)

With references, with summaries in English and Dutch

ISBN 978-94-6343-352-5

DOI 10.18174/460173 
Voor Leo 



\section{Table of contents}

\section{Chapter 1}

Introduction

Chapter 2

17

Expression of the Aspergillus terreus itaconic acid biosynthesis

cluster in Aspergillus niger

\section{Chapter 3}

Pathway transfer in filamentous fungi: Transporters are the key to success

\section{Chapter 4}

Overexpression of a modified 6-phosphofructo-1-kinase results in an

increased itaconic acid productivity in Aspergillus niger

\section{Chapter 5}

Funbricks: design of versatile expression vectors for synthetic biology

of Aspergillus niger

\section{Chapter 6}

85

Visual selection of targeted integration in Aspergillus niger

\section{Chapter 7}

Optimization strategies for microbial itaconic acid biosynthesis

\section{Chapter 8}

General discussion

\section{Appendices}

Summary

Samenvatting

Acknowledgements

List of publications

Overview of completed training activities 


\section{6}

(9)
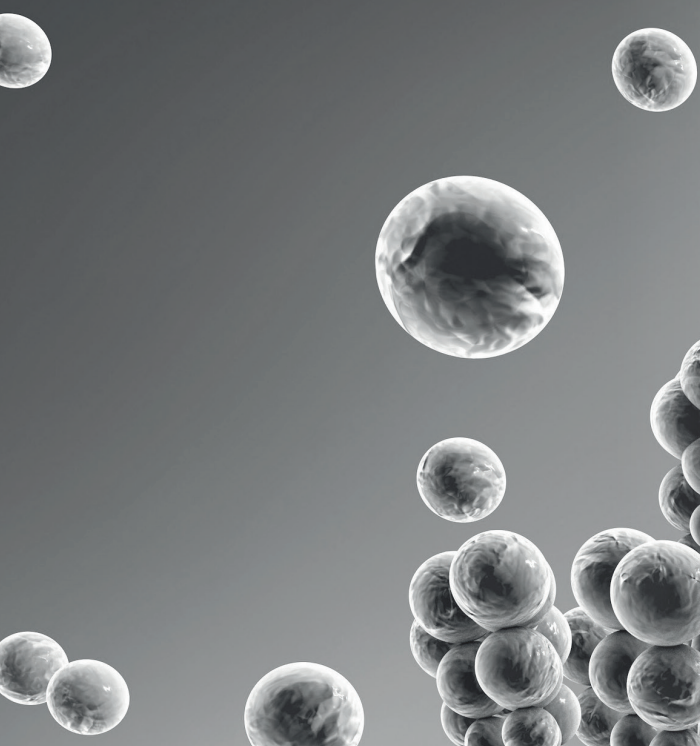

6

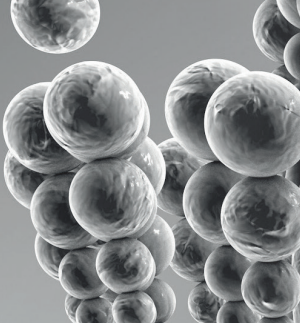

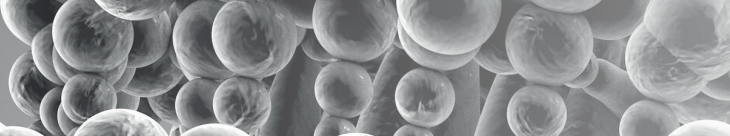
(1)
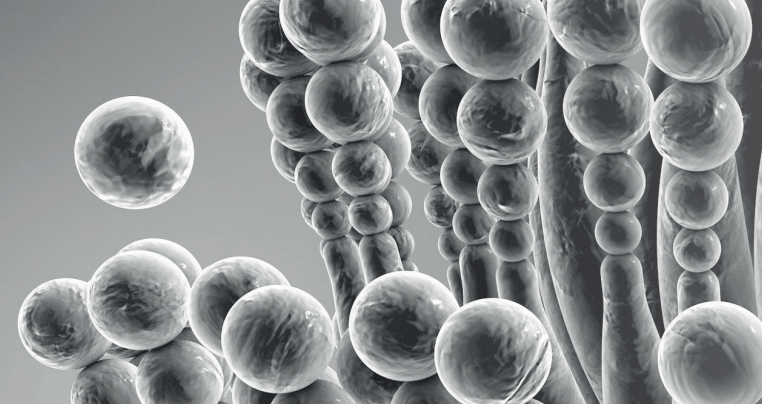

(9)
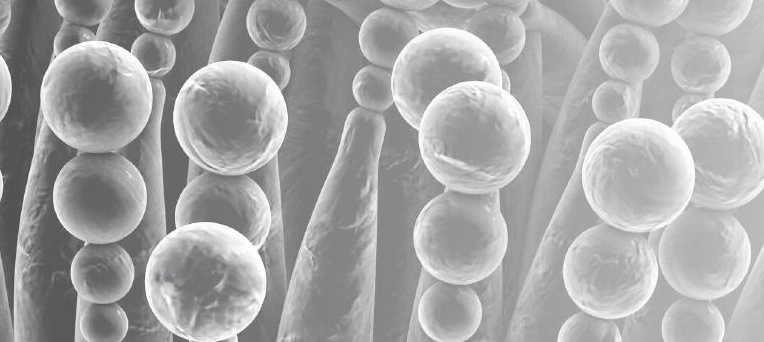

$$
\text { Q }
$$
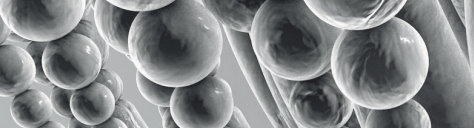

428

init 180

il mo

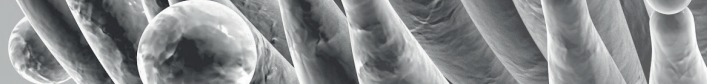

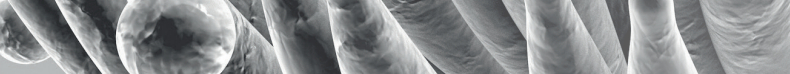

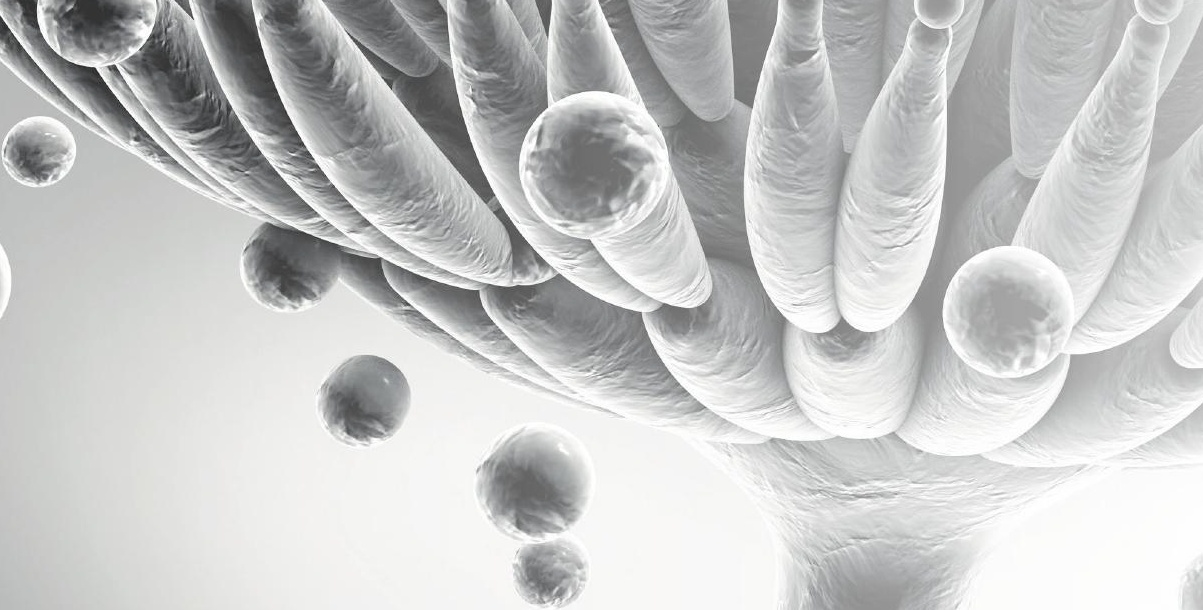

(.

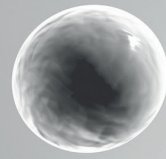

6 


\section{Chapter 1}

Introduction 
The current trend is to move from a petro-chemical based economy towards a circular economy, which intrinsically results from sustainable production processes. Part of this circular economy is the transition from petroleum-based production processes to bio-based production processes for e.g. chemicals, as illustrated in Fig. 1.1.

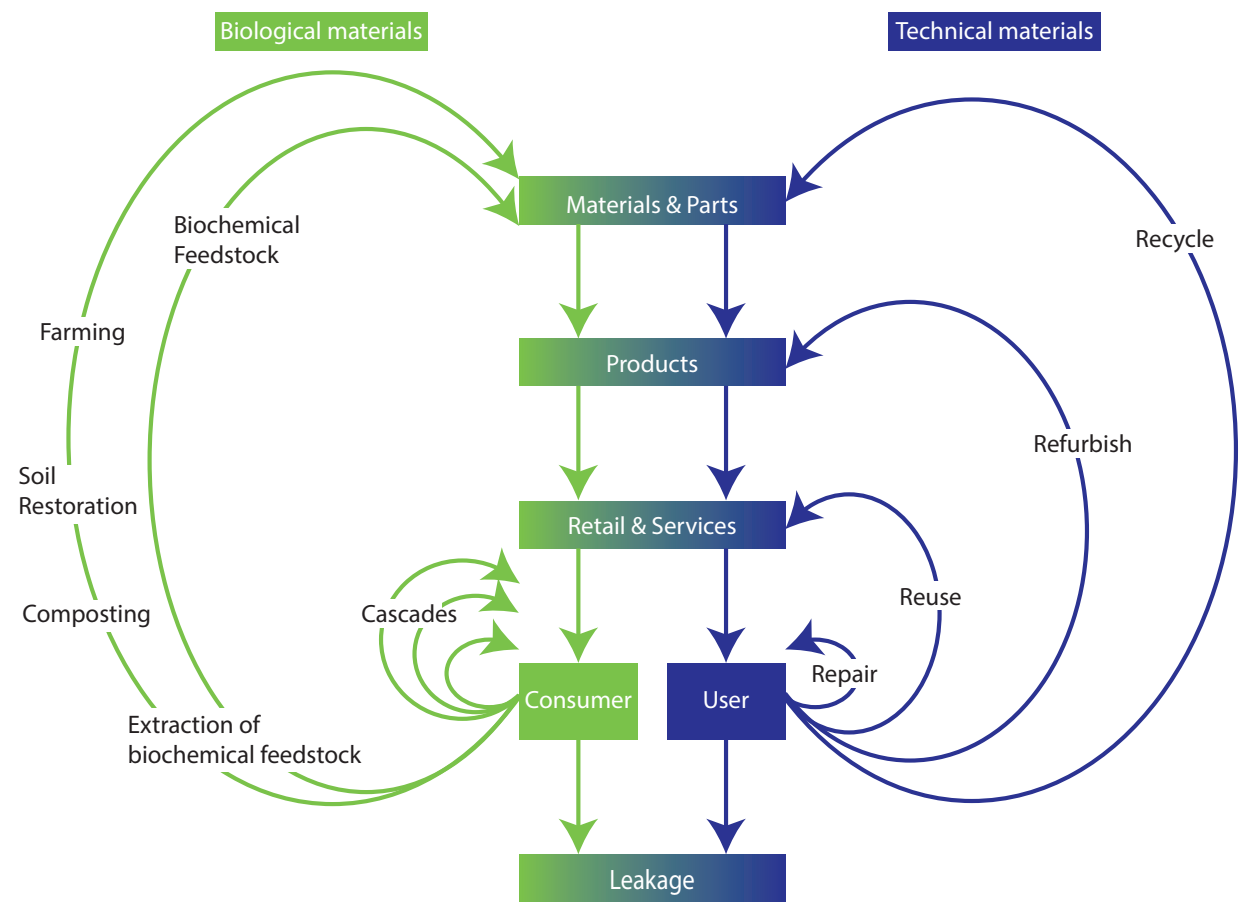

Figure 1.1 The circular economy aims to recycle both natural and technical materials thereby generating a minimum preferably zero waste or leakage. ${ }^{1,2}$

In 2004 the U.S. Department of Energy published a report on the top value added chemicals that can be produced from biomass. ${ }^{3}$ In this study twelve building block chemicals were identified (Table 1.1). These twelve building block chemicals all have the potential to be converted into high-value chemicals or materials and they can be produced from sugars via biological or chemical conversions. 
Table 1.1 The top twelve added-value building block chemicals determined by the US Department of Energy. ${ }^{3}$

\begin{tabular}{|c|c|}
\hline Building block chemical & Potential applications \\
\hline $\begin{array}{l}\text { 1,4-diacids } \\
\text { (succinic, fumaric, malic acid) }\end{array}$ & $\begin{array}{l}\text { - Solvents } \\
\text { - Water soluble polymers } \\
\text { - Fibers such as lycra }\end{array}$ \\
\hline 2,5-furandicarboxylic acid & $\begin{array}{l}\text { - PET analogs for the production of bottles, films and containers } \\
\text { - Polyesters and nylons }\end{array}$ \\
\hline 3-hydroxypropionic acid & $\begin{array}{l}\text { - } \text { Contact lenses } \\
\text { - Diapers }\end{array}$ \\
\hline Aspartic acid & $\begin{array}{l}\text { - Sweeteners } \\
\text { - Salts for chelating agent }\end{array}$ \\
\hline Glucaric acid & $\begin{array}{l}\text { - Solvents } \\
\text { - Nylons (like Kevlar) }\end{array}$ \\
\hline Glutamic acid & - Monomers for polyesters and polyamides \\
\hline Itaconic acid & $\begin{array}{l}\text { - } \text { Copolymers with styrene-butadiene polymers } \\
\text { - } \quad \text { Nitrile latex } \\
\text { - Solvents }\end{array}$ \\
\hline Levulinic acid & $\begin{array}{l}\text { - Fuel oxygenates } \\
\text { - Solvents } \\
\text { - Replacement for bisphenol A used in polycarbonate synthesis }\end{array}$ \\
\hline 3-hydroxybutyrolactone & $\begin{array}{l}\text { - Intermediate for high-value pharma compounds } \\
\text { - Solvents } \\
\text { - Amino analogs to lycro fibers }\end{array}$ \\
\hline Glycerol & $\begin{array}{l}\text { - } \text { Consumed in products such as personal/oral care products, drugs/ } \\
\text { pharmaceuticals, foods/beverages } \\
\text { - Antifreeze } \\
\text { - } \text { Polyester fibers }\end{array}$ \\
\hline Sorbitol & $\begin{array}{l}\text { - } \text { PET like polymers } \\
\text { - Antifreeze } \\
\text { - Water soluble polymers }\end{array}$ \\
\hline Xylitol/arabinitol & $\begin{array}{l}\text { - Non-nutritive sweeteners } \\
\text { - Antifreeze } \\
\text { - New polymer opportunities }\end{array}$ \\
\hline
\end{tabular}

One of these chemicals is itaconic acid (2-methylidenebutanedioic acid), a C5 dicarboxylic acid also known as methylenesuccinic acid. Itaconic acid can be processed into a polymer, which can be used to replace the petroleum-based polyacrylic acids. Polyacrylic acids are used in the production of for instance diapers, detergents, and cosmetics (Fig 1.2). 4,5 


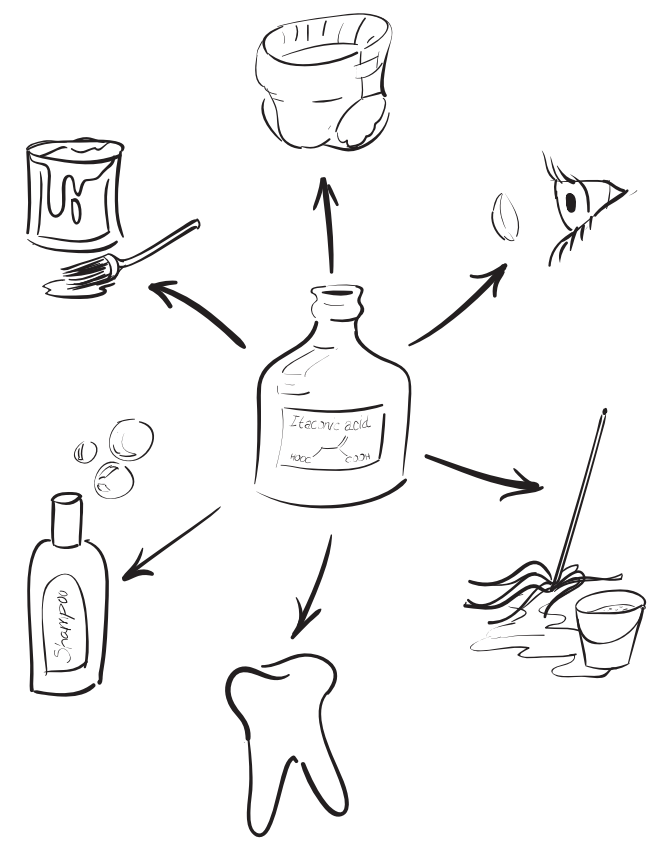

Figure 1.2 Itaconic acid and its potential applications in industry e.g. diapers, contact lenses, industrial cleaners, dental fillers, shampoos and additives for paint. ${ }^{5}$

Aspergillus niger is a filamentous fungus that is well-known for its ability to produce citric acid at an industrial scale. Citric acid titers over $200 \mathrm{~g} / \mathrm{L}$ are achieved. This characteristic is combined with the capacity to degrade plant cell wall polysaccharides. For these industrially interesting properties the organism has been investigated for more than 100 years. As the A. niger products have a long history of safe use in food industry these products have the GRAS status.

Nowadays, itaconic acid is mainly produced using the filamentous fungus Aspergillus terreus as a building block for specialty commodities. The maximum itaconic acid titer achieved using $A$. terreus so far is $86 \mathrm{~g} / \mathrm{L} .{ }^{6}$ In A. terreus citrate is converted into itaconate through the subsequent action of aconitase and cis-aconitate decarboxylase (CadA; Fig. 1.3). The key enzyme CadA competes with aconitase for the conversion of the intermediate product cis-aconitate. Hydration of cis-aconitate by aconitase would result in isocitrate (Fig. 1.3). 7,8

CadA (EC 4.1.1.6) belongs to the family of lyases, more specifically the carboxy-lyases. The enzyme catalyses the reaction whereby the carboxyl group is removed and $\mathrm{CO}_{2}$ is released. CadA shares high identity with proteins from the MmgE/PrpD family, which includes several bacterial 2-methylcitrate dehydratases. The cadA gene consists of 1529 bp encoding 490 amino acids and has only one intron. ${ }^{9}$ 


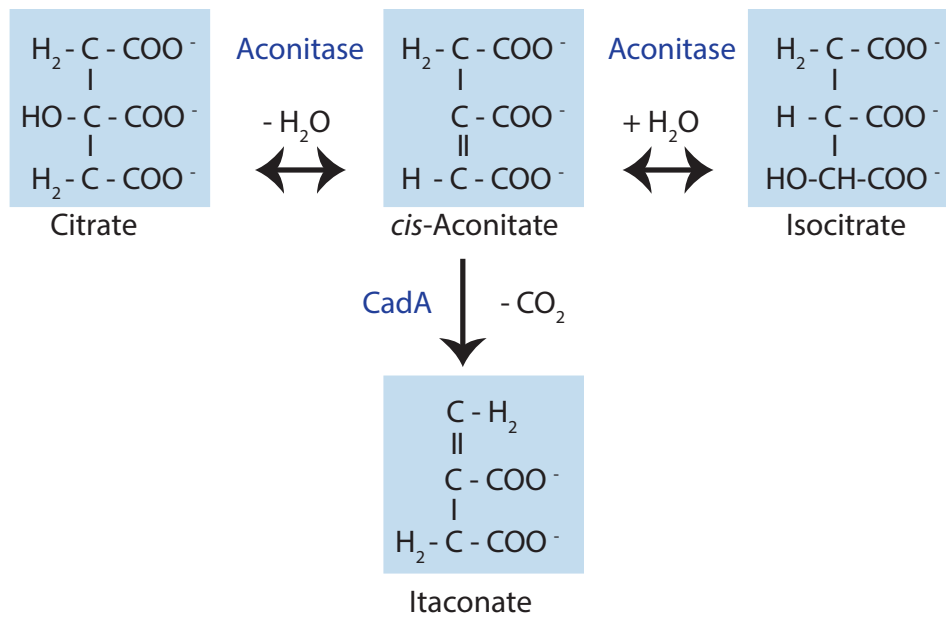

Figure 1.3 Metabolic reactions in A. terreus that lead to the synthesis of itaconate from citrate via cis-aconitate. The reactions are catalysed by aconitase and cis-aconitate decarboxylase (CadA). 7,8

Aconitase or aconitate hydratase (EC 4.2.1.3) catalyses the isomerization of citrate to isocitrate with the intermediate formation of cis-aconitate (Figure 1.3). This isomerization reaction is part of the tricarboxylic acid cycle (TCA cycle). In Aspergillus, a cytosolic form and a mitochondrial form are present. ${ }^{10}$

Based on the citric acid titers obtained in A. niger, using this organism as a production host for itaconic acid would make titers of around $135 \mathrm{~g} / \mathrm{L}$ itaconic acid feasible. This is far higher than the maximum levels of itaconic acid produced by $A$. terreus. This, combined with the ability to use agricultural plant waste as a substrate and the GRAS status of the products of A. niger, makes $A$. niger potentially a very interesting host for the biobased production of itaconic acid.

The key enzyme CadA is not present in A. niger and should therefore be introduced in order to re-route the metabolism to establish the production of itaconic acid.

In this thesis we have investigated $A$. niger for its potential as a host in itaconic acid production. Our strategy to achieve this was to:

1. introduce the gene encoding the key enzyme cis-aconitate decarboxylase (CadA)

2. study the role of transporters that are potentially involved in itaconic acid production

3. optimize the itaconic acid production by metabolic engineering 
Chapter 2 describes the actual transfer of the biosynthetic pathway of itaconic acid production from $A$. terreus towards $A$. niger. It shows that expression of the gene cadA encoding the enzyme cis-aconitate decarboxylase in $A$. niger leads to low levels of itaconic acid production. Expressing two genes encoding the putative transporters $m t t A$ and $m f s A$ that are flanking the $c a d A$ gene in the genome of $A$. terreus further enhanced the itaconic acid production. In Chapter 3, the challenges and opportunities of the expression of heterologous transporters is described.

Chapter 4 shows how to increase citric acid production levels and itaconic acid productivity levels by overexpression of a modified phosphofructokinase. Phosphofructokinase is a glycolytic enzyme that was modified in such a way that it is released from citrate inhibition. This resulted in enhanced citric acid production and enhanced itaconic acid productivity levels.

Chapter $\mathbf{5}$ and $\mathbf{6}$ describe tools that were developed to facilitate faster metabolic engineering and easy screening methods for transformants of $A$. niger.

In Chapter $\mathbf{5}$ the design of the Funbrick system is described and the itaconic acid biosynthesis cluster has been used as an example on how to use the Funbrick system. The Funbrick system comprises a family of expression vectors that can easily be adapted. In order to express pathways, several steps are usually needed to create the strain of interest. Using Funbrick vectors this can be done in a single transformation event. In this chapter the complete itaconic acid biosynthesis cluster from $A$. terreus consisting of cadA, mttA and $m f s A$ is transferred in one transformation event to $A$. niger. Also the localisation of the enzyme CadA and the two transporters MttA and MfsA were shown using Funbricks.

Chapter 6 describes an easy screening method that makes use of a change in spore color to quickly identify transformants that have integrated plasmid constructs of interest at a specific locus in the genome. The screening method exploits the fact that one single gene is responsible for the formation of fawn colored spores. Targeting the construct of interest to this particular locus results in a disruption of this particular gene fwnA. The disruption of the gene is immediately visible on the initial transformation plate since the color shifts from black to fawn.

In Chapter $\mathbf{7}$ the state-of-the-art of microbial itaconic acid production is presented. The biosynthetic pathway of $A$. terreus, a natural producer of itaconic acid, is described. Several strategies are summarized that have been applied to enhance itaconic acid production. On the one hand, optimizing itaconic acid production in the natural producers was done using metabolic engineering and fermentation optimization. On the other hand, different research groups investigated alternative hosts for designing a novel itaconic acid production process. The main focus of this chapter is on the strategies that were applied to A. niger as a potential itaconic acid producer. 
In Chapter 8, the results of this thesis are summarized and future perspectives are discussed. This thesis was part of the BE-Basic project "Novel economic and eco-efficient processes for the production of itaconic acid and fumaric acid" which was part of Flagship 1: Second Generation Carbon-based Compounds.

In our research group the production of itaconic acid and fumaric acid in A. niger was investigated. Another group from Wageningen University was responsible for the itaconic acid synthesis in Escherichia coli. Saccharomyces cerevisiae was investigated as a host for fumaric acid production at TU Delft. The downstream processing of both itaconic acid and fumaric acid was researched at TU Delft. During the course of the project, a complementary research project was initiated to further investigate the importance of transport processes in the production of organic acids by $A$. niger. This work has been carried out at Wageningen University and TU Delft. Finally, to obtain a holistic view on organic acid production in $A$. niger and relevant transport processes therefor, metabolic modeling combined with bioinformatics was performed at Wageningen University.

\section{References}

1. Pearce DW, Turner RK. Economics of Natural Resources and the Environment, Johns Hopkins University Press. 1990.

2. MacArthur E. Circular Economy, Ellen MacArthur Foundation. Available at: https://www. ellenmacarthurfoundation.org/ [Accessed May 5, 2016]

3. Werpy T, Petersen G. Top value added chemicals from biomass, 2004:1-76.

4. de Jong E, Higson A, Walsh P, Wellisch M. Product developments in the bio-based chemicals arena. Biofuel Bioprod Bioref 2012,6:606-624.

5. Willke T, Vorlop KD. Biotechnological production of itaconic acid. Appl Microbiol Biotechnol 2001,56: 289-295.

6. Kuenz A, Gallenmüller Y, Willke T, Vorlop KD. Microbial production of itaconic acid: developing a stable platform for high product concentrations. App/ Microbiol Biotechnol 2012,96:1209-1216.

7. Dwiarti L, Yamane K, Yamatani H, Kahar P, Okabe M. Purification and characterization of cisaconitic acid decarboxylase from Aspergillus terreus TN484-M1. J Biosci Bioeng 2002,94:29-33.

8. Bentley R, Thiessen CP. Biosynthesis of itaconic acid in Aspergillus terreus. III. The properties and reaction mechanism of cis-aconitic acid decarboxylase. J Biol Chem 1957,226:703-20.

9. Kanamasa S, Dwiarti L, Okabe O, Park EY. Cloning and functional characterization of the cisaconitic acid decarboxylase (CAD) gene from Aspergillus terreus. Appl Microbiol Biotechnol 2008,80:223-229.

10. Pel HJ, de Winde J, Archer DB, Dyer PS, Hofmann G, Schaap PJ et al. Genome sequencing and analysis of the versatile cell factory Aspergillus niger CBS 513.88. Nature Biotechnol 2007,25:221-231. 


\section{6}

(9)
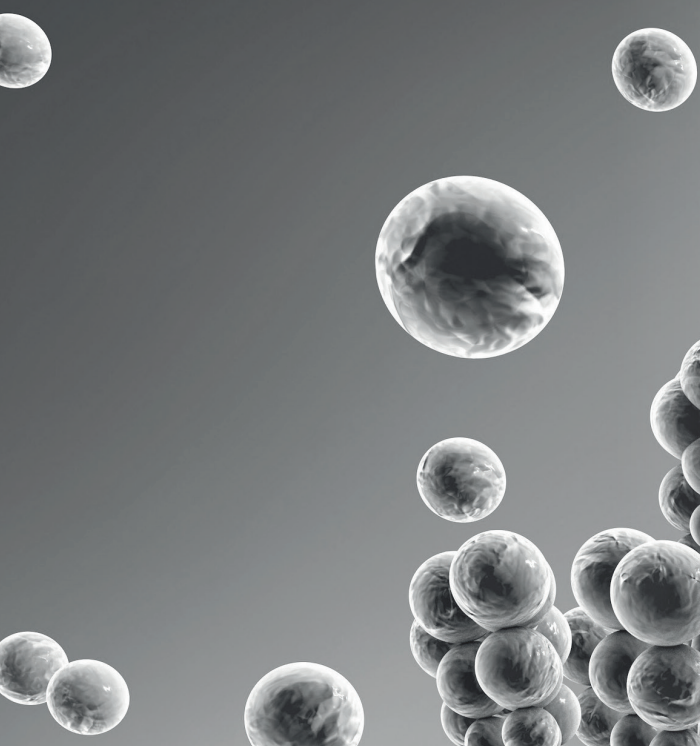

6

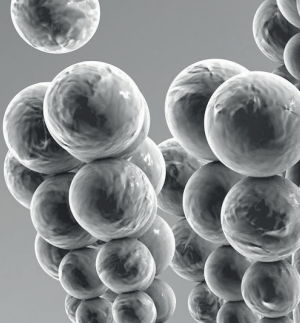

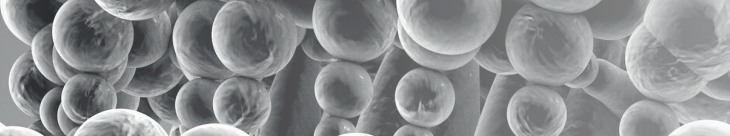
(1)
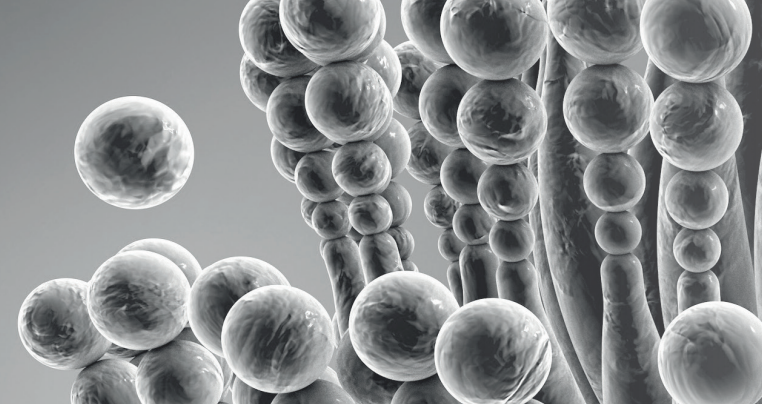

(9)
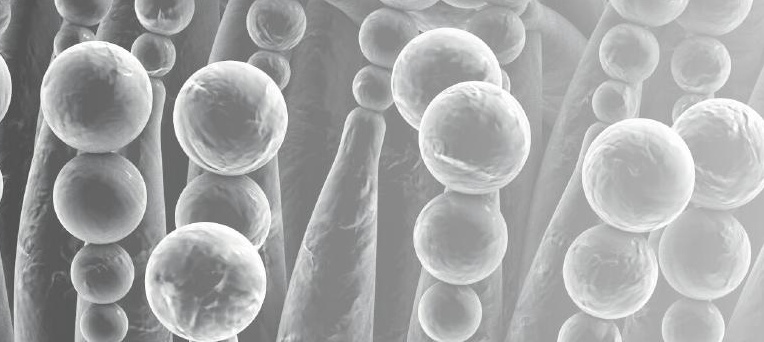

$$
\text { Q }
$$
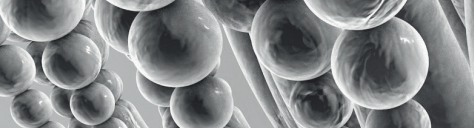

428

init 180

il mo

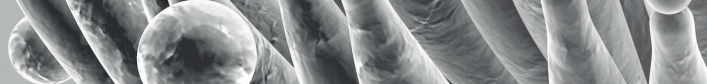

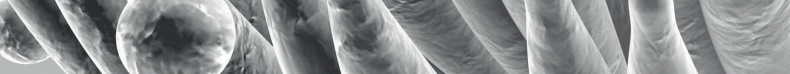

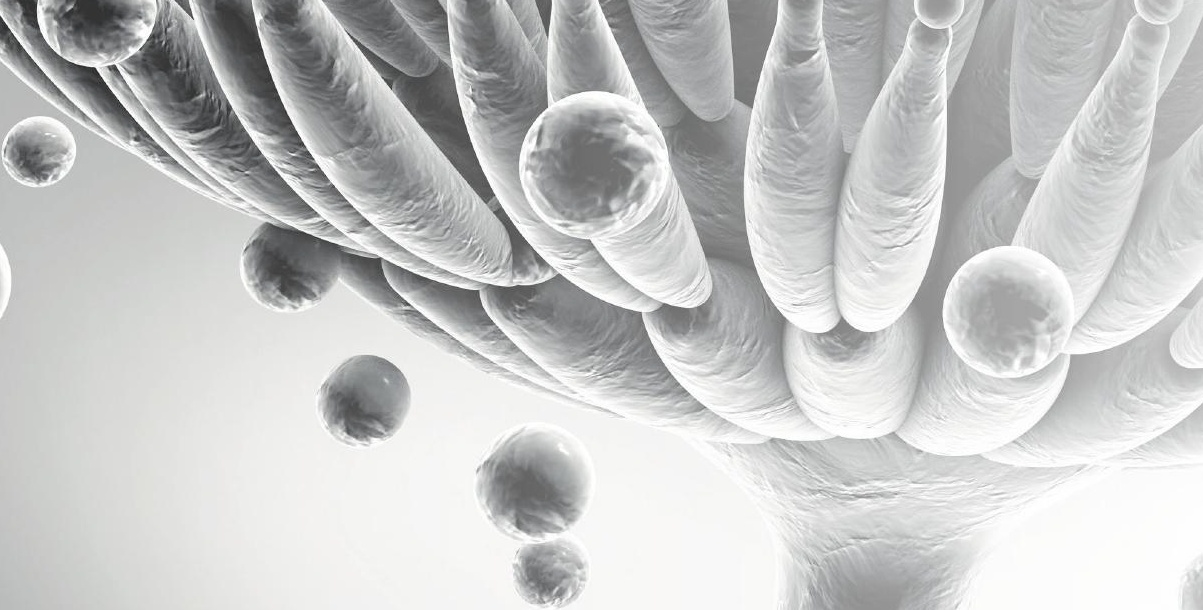

(.

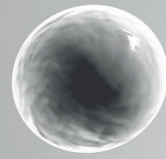

6 


\section{Chapter 2}

\section{Expression of the Aspergillus terreus itaconic acid biosynthesis cluster in Aspergillus niger}

L. van der Straat, M. Vernooij, M. Lammers, W. van den Berg, T. Schonewille, J. Cordewener, I. van der Meer, A. Koops and L.H. de Graaff. Expression of the Aspergillus terreus itaconic acid biosynthesis cluster in Aspergillus niger. Microbial Cell Factories, 2014,13:11 


\section{Abstract}

\section{Background}

Aspergillus terreus is a natural producer of itaconic acid and is currently used to produce itaconic acid on an industrial scale. The metabolic process for itaconic acid biosynthesis is very similar to the production of citric acid in Aspergillus niger. However, in A. niger, cisaconitate decarboxylase, the key enzyme for itaconic acid biosynthesis, is missing. Based on the high level of citric acid production (over $200 \mathrm{~g}$ per liter), introduction of the A. terreus cadA gene in $A$. niger theoretically can lead to production levels of over $135 \mathrm{~g}$ per liter of itaconic acid in A. niger. Given the potential for higher production levels in A. niger, production of itaconic acid in this host was investigated.

\section{Results}

Expression of Aspergillus terreus cis-aconitate decarboxylase in Aspergillus niger resulted in the production of a low concentration $(0.05 \mathrm{~g} / \mathrm{L})$ of itaconic acid. Overexpression of codonoptimized genes for cis-aconitate decarboxylase, a mitochondrial transporter and a plasma membrane transporter in an oxaloacetate hydrolase and glucose oxidase deficient $A$. niger strain, led to highly increased yields and itaconic acid production titers. At these higher production titers, the effect of the mitochondrial and plasma membrane transporters was much more pronounced, with levels being 5-8 times higher than previously described.

\section{Conclusions}

Itaconic acid can be produced in A. niger by the introduction of the A. terreus cis-aconitate decarboxylase encoding cadA gene. This results in a low itaconic acid production level, which can be increased by codon-optimization of the cadA gene for A. niger. A second crucial requirement for efficient production of itaconic acid is the expression of the $A$. terreus $m t t A$ gene, encoding a putative mitochondrial transporter. Expression of this transporter results in a twenty-fold increase in the secretion of itaconic acid. Expression of the $A$. terreus itaconic acid cluster consisting of the cadA gene, the $m t t A$ gene and the $m f s A$ gene results in $A$. niger strains that produce over twenty five-fold higher levels of itaconic acid and show a twenty-fold increase in yield compared to a strain expressing only CadA.

\section{Keywords}

Aspergillus niger, Aspergillus terreus, cis-aconitate decarboxylase cadA, mitochondrial transporter $m t t A$, plasma membrane transporter $m f s A$, itaconic acid 


\section{Background}

Increased awareness of the environmental pressure caused by petroleum-based production processes and products has stimulated and intensified research on bio-based production methods and products. Efficient bio-based production is economically problematic due to the relative low-cost of petroleum-based chemicals and is also technically complex. The design and construction of efficient cell factories requires a modification of the host cell or chassis at a systems level rather than at a single gene level.

Itaconic acid or methylsuccinic acid is a C5 dicarboxylic acid. The methylene group of itaconic acid can participate in polymerization reactions. On the basis of this characteristic, itaconic acid can be used for the production of synthetic polymers. ${ }^{1}$ Furthermore, it can be used as a bioactive component in agriculture and pharmacy, as a medicine ${ }^{2}$, and as a starting compound in enzymatic conversions to form useful poly-functional building blocks. ${ }^{3}$ For all of these reasons, itaconic acid has been designated by the U.S. Department of Energy as one of the top twelve building-block chemicals that can be produced from plant biomass sugars via a fermentative process. ${ }^{4}$

Currently, A. terreus is used for the commercial production of itaconic acid by submerged fermentation. 2,5 The pathway for the production of itaconic acid is a metabolic variant of the pathway for citric acid production in A. niger (Fig. 2.1).

Citric acid is produced commercially using A. niger, reaching production levels over 200 $\mathrm{g} / \mathrm{L}{ }^{6}$, which, in a molar ratio, corresponds to over $135 \mathrm{~g} / \mathrm{L}$ itaconic acid. A. terreus reaches itaconic acid titers of $80 \mathrm{~g} / \mathrm{L}$ showing the potential of $A$. niger to reach far higher production titers. A second advantage is that the existing citric acid fermentation infrastructure can be used for this A. niger-based fungal itaconic acid cell factory.

A. niger does not naturally produce itaconic acid because it lacks the essential enzyme cis-aconitate decarboxylase. The cadA gene encoding this enzyme in A. terreus has been identified using different approaches, including enzyme purification ${ }^{7}$ and clone-based transcriptomics. ${ }^{8}$ The expression of the cadA gene in $A$. niger leads to extremely low levels of itaconic acid production $(0.05 \mathrm{~g} / \mathrm{L})$, indicating that the sole expression of the enzyme is insufficient for efficient production of itaconic acid. In the A. terreus genome, the cadA gene is located close to the lovastatin cluster ${ }^{9}$ and is flanked by a putative mitochondrial transporter ( $m t t A)$ and a putative plasma membrane transporter $(m f s A)$. The co-regulation of these transporters with $\operatorname{cad} A$, as reported by Li et al. ${ }^{8}$, suggested that the putative mitochondrial transporter might be involved in itaconic acid production in A. terreus. Recently, Li et al. ${ }^{10}$ showed that the effect of these putative transporters on itaconic acid production in $A$. niger resulted in a slight increase in itaconic acid production levels. However, 
the maximum titer of $1.5 \mathrm{~g} / \mathrm{L}$ itaconic acid that was reached is far from the theoretical titer of over $135 \mathrm{~g} / \mathrm{L}$ under conditions of high citric acid production.

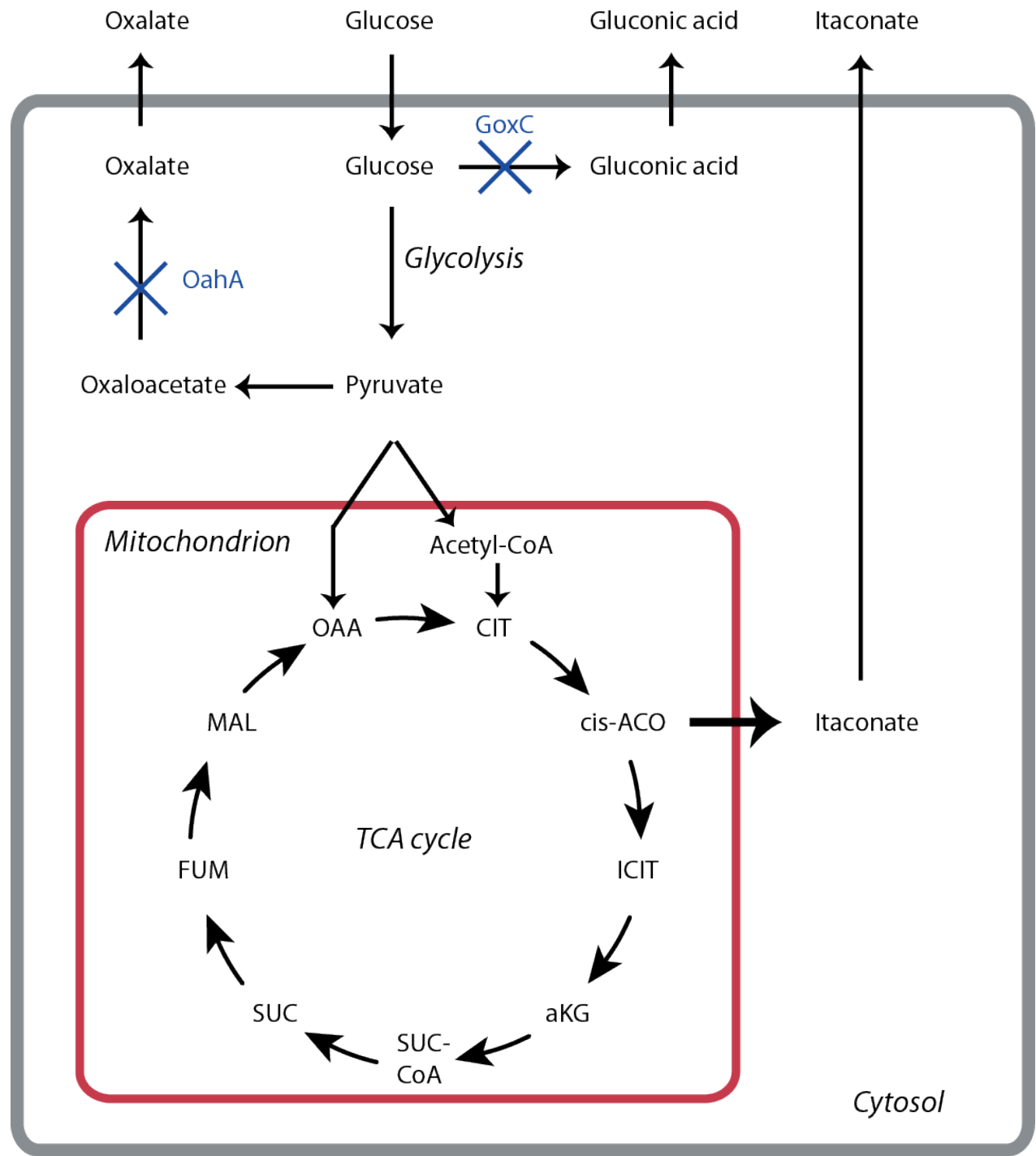

Figure 2.1 Metabolic pathway for itaconic acid production via the different compartments in the specific $A$. niger strain.

For our studies, we have used a specific mutant of $A$. niger to serve as a chassis for the production of itaconic acid. This strain carries two specific mutations, one in the oahA gene encoding oxaloacetate hydrolase and one in the gox $C$ gene encoding glucose oxidase. This strain has certain advantages; the production of by-products is reduced because it is not 
able to produce oxalic acid or gluconic acid. As a result, this leaves more carbon available for citrate and itaconate production. Finally, as reported by Ruijter et al., strains carrying both the oahA mutation and the goxC mutation are insensitive to $\mathrm{Mn}^{2+}$ ions in the medium, which results in constitutive citrate production irrespective of the fermentation regime. ${ }^{11}$ In this study, we use a robust fermentation regime developed by van der Veen et al. ${ }^{12}$ that is optimized to reduce variance in the experiments. In the fermentation medium, sorbitol is used as the main carbon source as it is essentially non-inducing and non-repressing for the $D$-xylose inducible $x \operatorname{InD}$ promoter. ${ }^{13}$ Sorbitol is metabolized to form fructose ${ }^{14}$, which is phosphorylated by hexokinase to fructose-6-phosphate and further metabolized via the glycolysis pathway and the TCA cycle. In the TCA cycle, citrate is converted into isocitrate in a reaction that yields cis-aconitate as an intermediate.

Itaconic acid can be formed from cis-aconitate by a cis-aconitate decarboxylase-catalyzed reaction.

In our study, we show that the overexpression of the codon-optimized cadA, $m t t A$ and $m f s A$ genes in the oxaloacetate hydrolase- and glucose oxidase-deficient strain leads to increased yields and itaconic acid production titers. At these higher production titers, the effect of the mitochondrial and plasma membrane transporters is much more pronounced than previously described. ${ }^{10}$

\section{Results and discussion}

Our strategy for the design of a fungal cell factory was based on the use of a specific chassis for the production of itaconic acid in $A$. niger. The A. niger strain that we chose is a mutant strain that is not able to produce oxalic acid or gluconic acid due to mutations in the oahA and gox $C$ genes, respectively. This is an important advantage because this strain does not produce these unwanted side products. Due to the reduced formation of by-products, more carbon can be converted into the final product - itaconic acid. The oahA mutation also leads to constitutive citric acid production that is insensitive to the presence of metal ions, as discovered by Ruijter et al. ${ }^{11}$ The constitutive production of citric acid is a great benefit because itaconic acid production is directly derived from citric acid production.

\section{Expression of the A. terreus cadA gene in A. niger}

The gene encoding cis-aconitate decarboxylase was identified in the $A$. terreus genome using a proteomics approach in which the enzyme was partially purified. Both a cDNA fragment from $A$. terreus and a codon-optimized cadA synthetic gene were used for the expression of cis-aconitate decarboxylase in A. niger NW186. The A. terreus coding sequences have a slightly higher GC content in comparison to the A. niger coding sequences ( $56.2 \%$ vs $53.8 \%$, respectively). ${ }^{15}$ This higher $\mathrm{GC}$ content is mostly found at the third position; in A. terreus, 
65.3\% GC and in A. niger, 59.3\%. A total of 305 out of 490 codons were changed in the cadA sequence, including the codons to remove restriction enzyme sites.

The transformants from both plasmids yielded varying low amounts of itaconic acid.

This variation in itaconic acid production could result from differences in copy numbers amongst the strains and variation in the site of integration of the construct in the different transformants. Surprisingly, codon-optimization of the cadA gene for $A$. niger resulted in a more than three-fold increase in itaconic acid production. The transformants containing the codon-optimized gene (SCAD) (Fig. 2.2B) produced higher amounts of itaconic acid compared to the ones expressing the cDNA fragment (cCAD) (Fig. 2.2A).
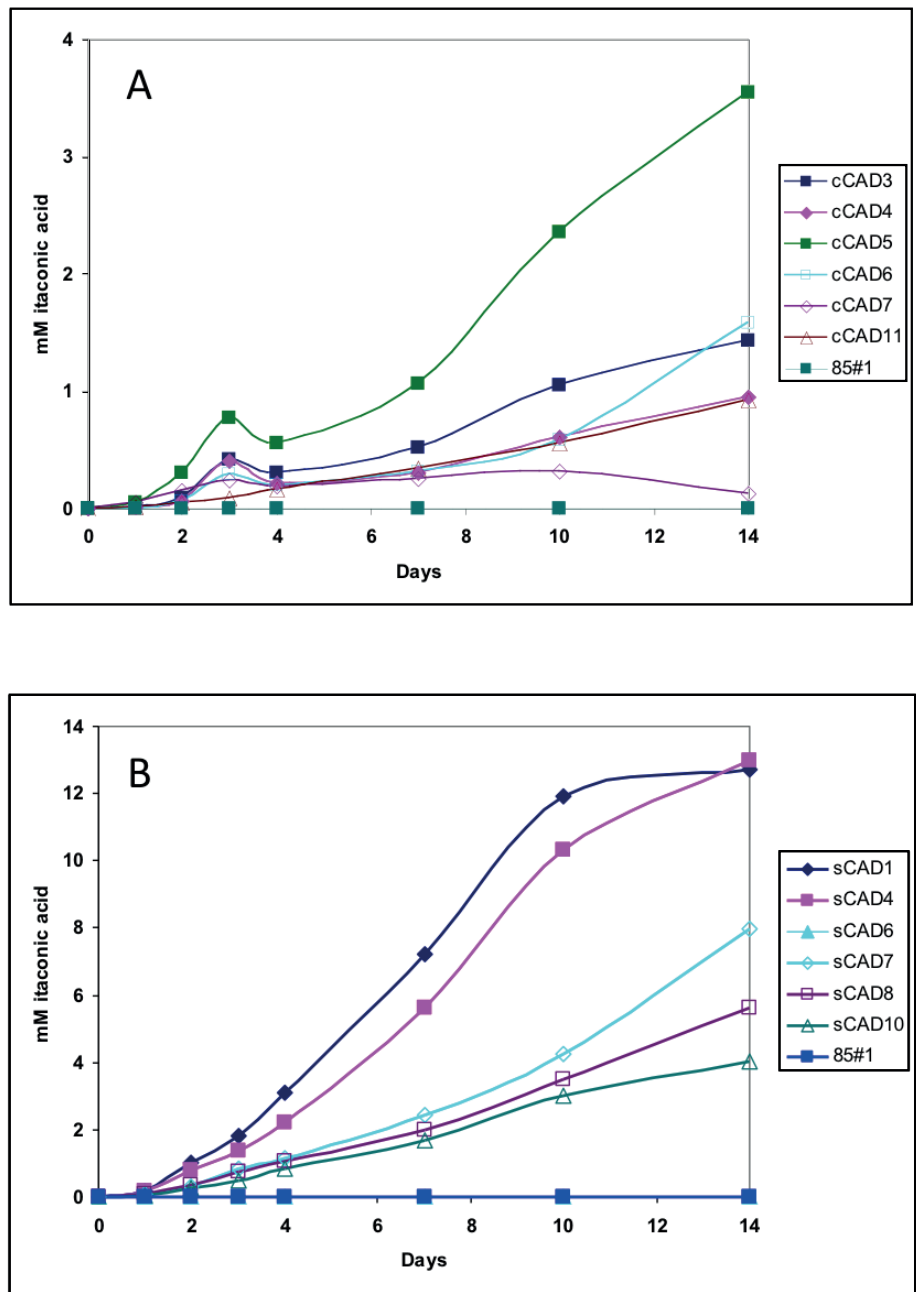

Figure $\mathbf{2 . 2}$ Itaconic acid production in A. niger strains expressing cis-aconitate decarboxylase. Production of itaconic acid (mM) in strains expressing cadA cDNA (cCAD) (A) and production of itaconic acid ( $\mathrm{mM}$ ) in strains expressing codon-optimized cadA synthetic DNA (SCAD)(B). 
Based on these results, the two putative transporters $m t t A$ and $m f s A$ from $A$. terreus were also synthetically made and codon-optimized for expression in $A$. niger.

No itaconic acid production was detected in the A. niger strains that did not contain the cis-aconitate decarboxylase encoding gene. The SCAD4 strain was selected for our further studies.

\section{Copy number of the cadA gene in $A$. niger transformants}

The copy number was determined by qPCR using genomic DNA as template. The Pfaffl method was applied to calculate the copy number. ${ }^{16}$ The qPCR results for $\operatorname{cad} A$ were compared with those of the single copy gene pkiA in order to determine the copy number of cadA. For the transformants CCAD4, cCAD6 and CCAD11, expressing the A. terreus cadA cDNA, a copy number of 1 was determined. These strains also produced the same levels of itaconic acid for 10 days. After 14 days, the cCAD6 strain produced a higher level of itaconic acid. Of the strains expressing the cadA cDNA, cCAD3 and CCAD5 had the highest copy number at 4 . These strains also produced the highest levels of itaconic acid of the strains that expressed the non-optimized cadA cDNA. The only atypical transformant was cCAD7, which had a copy number of 21 but produced hardly any itaconic acid. Although this was a striking result, it has previously been observed in A. niger. ${ }^{17}$

The highest itaconic acid producing transformants, sCAD1 and SCAD4, expressed the synthetic codon-optimized cadA gene and had the highest copy numbers at 11 and 6, respectively. The transformants SCAD7, SCAD8 and SCAD10, which produced between 4 and $8 \mathrm{mM}$ itaconic acid after 14 days, had copy numbers of 2, 2 and 4, respectively. Although the sCAD10 strain had 4 copies of cadA, it did not produce more than the strains with only 2 copies of $c a d A$. In this particular case, the place of integration could negatively influence the level of expression compared to SCAD7 and SCAD8. The SCAD6 strain, which did not produce any itaconic acid, also did not have a copy of the cadA gene.

These results also suggest a positive effect of codon-optimization because the strains that produced the highest levels of itaconic acid, namely sCAD7, sCAD8 and sCAD10, were the strains expressing the codon-optimized cadA gene.

The copy number of these strains was determined to be 2, 2 and 4, respectively. This is in contrast to the CCAD5 and CCAD3 strains, which carried 4 copies of the non-optimized cadA gene but produced less itaconic acid.

\section{Expression of the $\boldsymbol{A}$. terreus itaconic acid biosynthesis cluster in $\boldsymbol{A}$. niger}

Based on our findings on the expression of the cadA in $A$. niger, we extended our studies by co-expressing the two putative transporter encoding genes flanking the cadA gene in the $A$. terreus genome. In these studies, we also used synthetic codon-optimized fragments of the $m t t A$ and $m f S A$ genes for expression in the $A$. niger strain that contains the codon-optimized cadA gene, sCAD4. For our first analysis, these strains were grown in Erlenmeyer culture flasks to analyze the effects of the transporters that were introduced. In these experiments, 
strains that contained the cadA and $m f s A$ genes produced slightly higher levels of itaconic acid compared to the SCAD4 strain carrying only the cadA gene (Table 2.1).

Table 2.1 Production of itaconic acid in Erlenmeyer cultures. The itaconic acid production is given in $\mathrm{mM}$ in the CadA + MfsA and CadA + MttA transformants at 30 hours after induction. Measurements were carried out in duplicate; the \pm represents the standard error of the mean.

\begin{tabular}{|c|c|c|}
\hline Transformants & Itaconic acid produced (mM) & Factor difference \\
\hline Control & $0.13 \pm 0.02$ & \\
\hline$c a d A+m f s A 2.4$ & $0.14 \pm 0.01$ & 1 \\
\hline$c a d A+m f s A 2.5$ & $0.25 \pm 0.06$ & 2 \\
\hline $\operatorname{cad} A+m t t A 1.1$ & $0.98 \pm 0.09$ & 8 \\
\hline $\operatorname{cad} A+m t t A 1.2$ & $3.23 \pm 0.94$ & 25 \\
\hline $\operatorname{cad} A+m t t A 1.4$ & $0.74 \pm 0.04$ & 6 \\
\hline$c a d A+m t t A 1.5$ & $0.74 \pm 0.00$ & 6 \\
\hline $\operatorname{cad} A+m t t A 1.6$ & $1.21 \pm 0.24$ & 10 \\
\hline
\end{tabular}

The putative mitochondrial transporter mttA had a much more pronounced effect on itaconic acid production, as expression of this gene led to increased itaconic acid production in all transformants analyzed in comparison to the strain that had only the cadA gene.

The increase found ranged between a factor of 6 and 25 (Table 2.1), which is far more than the previously described increase for an $A$. niger strain carrying only the oahA mutation and in which the genes were not codon-optimized. ${ }^{10}$

We performed batch fermentations to study the improved itaconic acid production in a more controlled way. To investigate the effect of the $m t t A$ and $m f s A$ transporters on the production of itaconic acid in $A$. niger, the best performing transformant of the $\operatorname{cad} A+m t t A$ and $c a d A+m f s A$ strains were selected along with four transformants of newly constructed strains carrying the combination of cadA, $m t t A$ and $m f s A$. The parent strain sCAD4 was chosen as the control.

When grown in batch fermenters, we did not find the slight increase in itaconic acid production in the strains that co-expressed $m f s A$ with cadA (Table 2.1, Fig. 2.3, Fig. 2.4) that we found in the Erlenmeyer cultures. This was in contrast to previously published data in which nearly five-fold higher itaconic acid levels were found when cadA was co-expressed with $m f s A .{ }^{10}$ However, we did find increased citric acid concentrations (Fig. 2.4). 


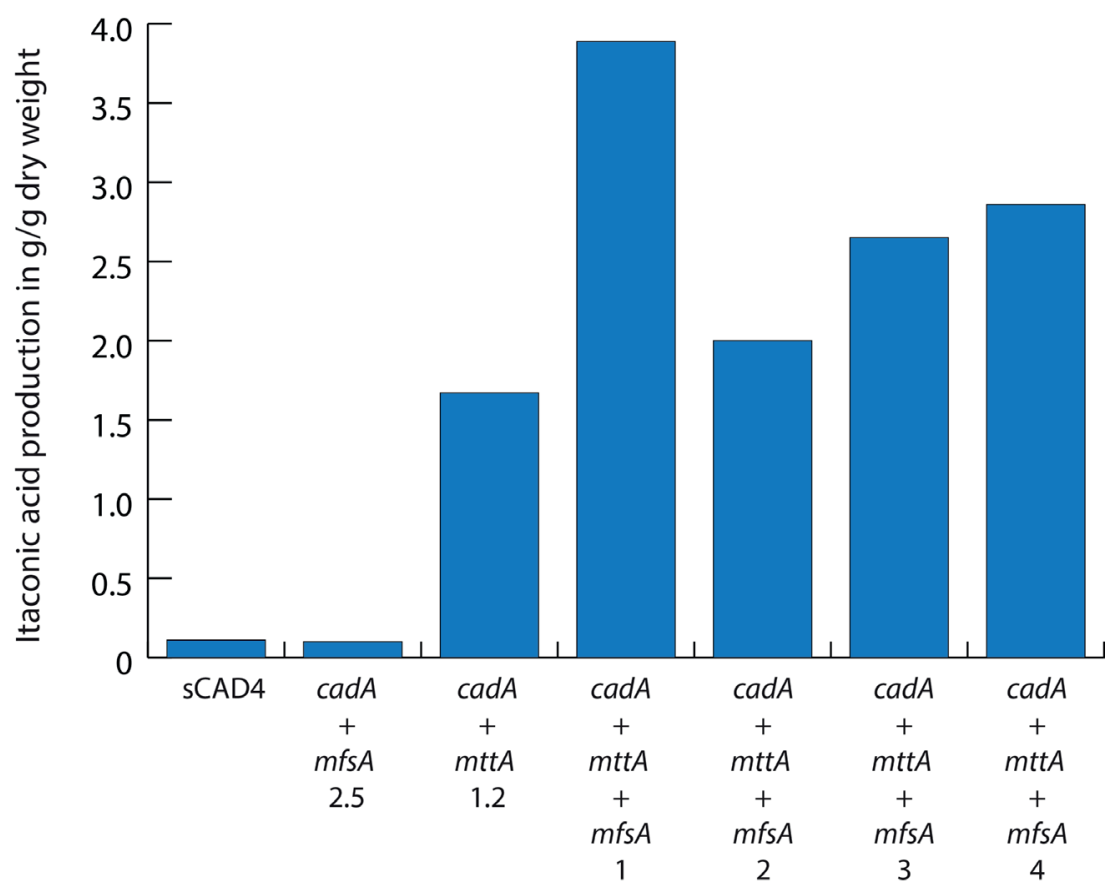

Figure $\mathbf{2 . 3}$ Itaconic acid production in fermenter cultures. The itaconic acid production is given in gram per gram dry weight at $\mathrm{t}=$ 78 hours after induction of the transformants carrying the complete itaconic acid biosynthesis cluster from $A$. terreus compared to the best performing CadA, CadA+MfsA and CadA+MttA transformants.
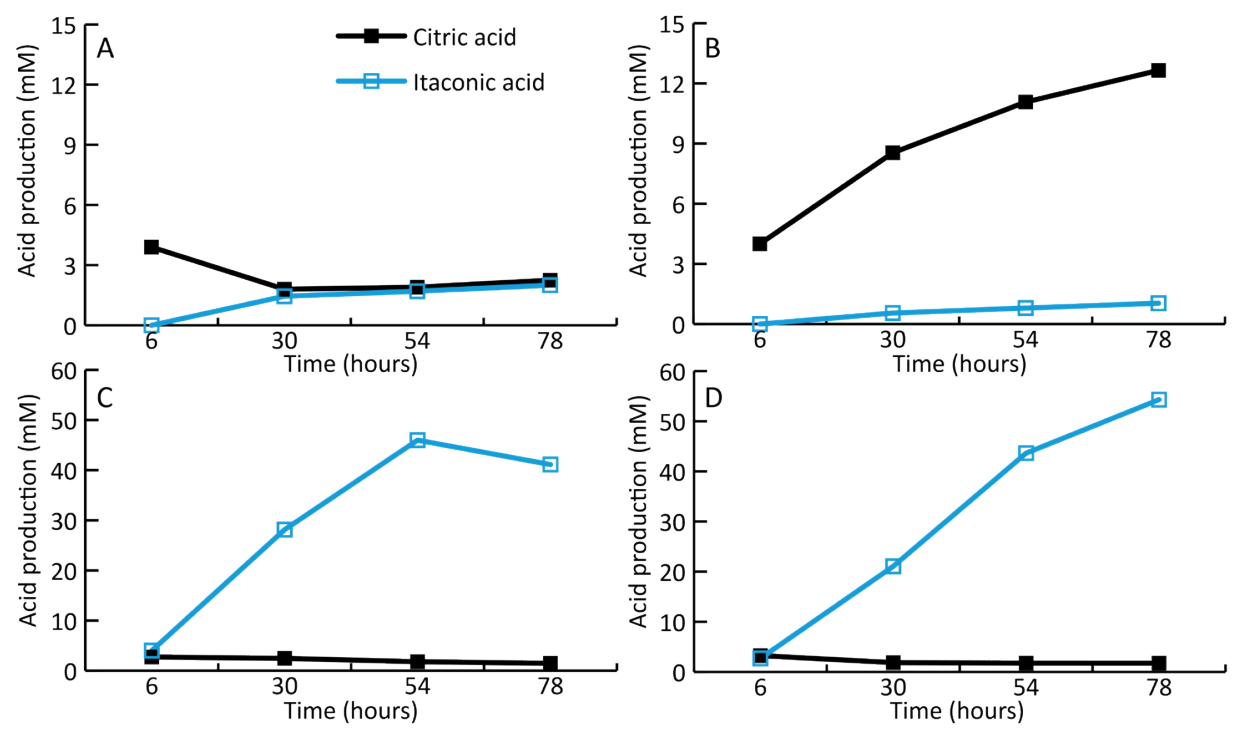

Figure 2.4 Itaconic acid and citric acid production during fermentation of the different strains. A) sCAD4 strain, B) $c a d A+m f s A 2.5$, C) $c a d A+m t t A 1.4$ and D) $c a d A+m t t A+m f s A$ 4. The black lines represent citric acid concentration and the blue line represents the itaconic acid concentration. The time is shown in hours ( $\mathrm{x}$-axis) and the concentrations are shown in mM (y-axis). 
This implies that this transporter is also able to export citric acid. Although we did not find a positive effect on the levels of itaconic acid production from strains expressing $m f s A$ and $\operatorname{cad} A$, we did find a positive effect on itaconic acid production levels in strains expressing cadA, mttA and mfsA in NW186 (Table 2.1, Fig. 2.3 and 2.4). Apparently, the plasma membrane transporter MfsA is able to secrete both itaconic acid and citric acid. Obviously, $A$. niger is able to secrete itaconic acid without a heterologous plasma membrane transporter as is shown by the expression of cadA in $A$. niger. The transformant cadA $+m f s A 2.5$ did not show increased itaconic acid production in the fermenter studies. However, because the itaconic acid levels are relatively low, it is possible that there was no bottleneck in itaconic acid export in this strain under these conditions.

In the case of the cadA + mttA + mfsA transformants, levels of itaconic acid production were strongly increased in comparison to the strain that only carried the cadA gene, with the highest increase being over 25-fold (Fig. 2.3).

These increased production levels were paralleled with an increased yield, defined as the product yield on the substrate in \% (C-mol/C-mol), in the transformants that contained both putative transporters. The strain expressing only cadA gave a yield of approximately $1 \%$, which is of the same order as was found by Li et al. ${ }^{10}$, although they used different carbon sources and different concentrations of carbon sources. The strongest increase in yield was caused by the expression of the mitochondrial transporter mttA in the SCAD4 strain, which resulted in a yield of $24 \%$. However, when the complete itaconic acid cluster from $A$. terreus was expressed, the yield further increased to $32 \%$ in the best performing strain (Table 2.2).

Table 2.2 Comparison of itaconic acid producing strains. The production of itaconic acid is given in $\mathrm{g} / \mathrm{L}$ at 78 hours after induction. The yield in percentage (C-mol/C-mol) is calculated based on consumed sorbitol and D-xylose. The last four strains are uridine prototrophs of $A B 1.13$ (data from Li et al. ${ }^{13}$ ).

\begin{tabular}{|c|c|c|c|c|}
\hline Strain & $\begin{array}{l}\text { Itaconic acid } \\
\text { production } \\
\text { (g/L) }\end{array}$ & $\begin{array}{c}\text { Sorbitol and } \\
\text { xylose consumed } \\
\text { (C-mmol) }\end{array}$ & $\begin{array}{l}\text { Itaconic acid yield } \\
\text { on consumed } \\
\text { sorbitol and } \\
\text { xylose (\%) }\end{array}$ & $\begin{array}{c}\text { Biomass } \\
\text { (g dry weight } \\
\text { per L culture } \\
\text { broth) }\end{array}$ \\
\hline sCAD4 & 0.3 & 619 & 1.6 & 2.4 \\
\hline $\operatorname{cad} A+m f s A 2.5$ & 0.1 & 632 & 0.8 & 1.2 \\
\hline $\operatorname{cad} A+m t t A 1.2$ & 5.4 & 843 & 24.4 & 3.2 \\
\hline$c a d A+m t t A+m f s A 1$ & 5.6 & 806 & 26.8 & 1.6 \\
\hline$c a d A+m t t A+m f s A 2$ & 6.0 & 848 & 27.4 & 3.1 \\
\hline$c a d A+m t t A+m f s A 3$ & 5.5 & 702 & 30.0 & 2.1 \\
\hline$c a d A+m t t A+m f s A 4$ & 7.1 & 844 & 32.1 & 2.6 \\
\hline AB 1.13 CAD & 0.9 & & 1.0 & \\
\hline MTT 1.4 & 1.4 & & 1.6 & \\
\hline MFS 3.9 & 1.4 & & 1.6 & \\
\hline CAD + MTT + MFS_3 & 0.9 & & 1.0 & \\
\hline
\end{tabular}


Although several other acids including cis-aconitic acid, succinic acid, malic acid, pyruvic acid and $\alpha$-ketoglutaric acid are secreted, itaconic acid was the predominantly produced acid in the highest producing strains. One exception was the strain expressing $\operatorname{cad} A$ in combination with $m f s A$ where, instead of itaconic acid, citric acid was the predominantly produced acid (Table 2.3).

Table 2.3 Overview of extracellular acid concentrations $(\mathrm{mM})$ measured in time during fermentation. -: the compound was not detected in the sample.

\begin{tabular}{|c|c|c|c|c|c|c|c|c|}
\hline & & 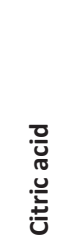 & 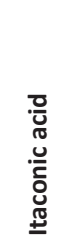 & 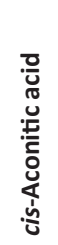 & 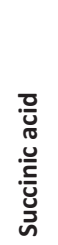 & 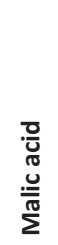 & 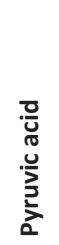 & 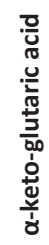 \\
\hline \multirow[t]{4}{*}{$6 \mathrm{~h}$} & sCAD4 & 3.9 & - & - & - & - & - & - \\
\hline & $c a d A+m f s A 2.5$ & 4.0 & - & - & - & - & - & - \\
\hline & $c a d A+m t t A 1.2$ & 2.7 & 4.0 & - & 0.2 & - & - & - \\
\hline & $c a d A+m t t A+m f s A 4$ & 3.3 & 2.7 & - & - & - & - & - \\
\hline \multirow[t]{4}{*}{$30 \mathrm{~h}$} & sCAD4 & 1.8 & 1.45 & 0.3 & - & - & 2.4 & - \\
\hline & $\operatorname{cad} A+m f s A 2.5$ & 8.5 & 0.6 & - & - & - & - & - \\
\hline & $\operatorname{cad} A+m t t A 1.2$ & 2.5 & 28.1 & - & 0.7 & - & - & 1.5 \\
\hline & $\operatorname{cad} A+m t t A+m f s A 4$ & 1.9 & 21.1 & 1.2 & - & - & 0.8 & - \\
\hline \multirow[t]{4}{*}{$54 \mathrm{~h}$} & sCAD4 & 1.9 & 1.7 & 0.3 & - & - & 2.1 & - \\
\hline & $\operatorname{cad} A+m f s A 2.5$ & 11.1 & 0.8 & - & 0.8 & 0.7 & - & - \\
\hline & $\operatorname{cad} A+m t t A 1.2$ & 1.8 & 46.0 & - & 1.4 & - & - & 0.4 \\
\hline & $c a d A+m t t A+m f s A 4$ & 1.8 & 43.6 & 1.8 & - & - & 0.6 & - \\
\hline \multirow[t]{4}{*}{$78 \mathrm{~h}$} & sCAD4 & 2.3 & 2.0 & 0.3 & - & - & 2.2 & - \\
\hline & $\operatorname{cad} A+m f s A 2.5$ & 12.7 & 1.0 & - & 0.9 & 0.7 & - & - \\
\hline & $\operatorname{cad} A+m t t A 1.2$ & 1.5 & 41.1 & - & 0.9 & - & - & - \\
\hline & $c a d A+m t t A+m f s A 4$ & 1.8 & 54.3 & 2.2 & 0.9 & - & - & - \\
\hline
\end{tabular}

The itaconic acid production levels obtained were relatively low compared to those in industrial production processes. It is not surprising that our strain was less efficient in itaconic acid production than the commercial $A$. terreus strain since the $A$. niger strain used is not producing high levels of citrate. The citrate concentrations produced by our strain are far lower than those obtained in an industrial environment using an industrial $A$. niger strain. Although itaconic acid production in $A$. niger was still far less efficient than in $A$. terreus, major improvements were made. Under lab conditions, we were able to improve the titer of itaconic acid produced in A. niger by a factor of over twenty-five and the yield by approximately twenty-fold. Compared to the values for itaconic acid production in 
A. niger that have been published ${ }^{10}$, the strains we constructed showed a five-fold higher production level and a twenty-fold increase in yield.

These improvements mainly resulted from the use of codon-optimized genes and from the increased efficiency of substrate-use by the elimination of oxalate and gluconate production.

\section{Conclusions}

Itaconic acid can be produced in A. niger by the introduction of the A. terreus cis-aconitate decarboxylase encoding cadA gene. However, this results in very low production levels. The production levels can be increased if the $A$. terreus cadA gene is codon-optimized for $A$. niger.

When the expression of cadA in $A$. niger was combined with the expression of the $A$. terreus $m f s A$ gene encoding a putative plasma membrane transporter, no effect on the production levels of itaconic acid was found. This suggests that the itaconic acid produced in $A$. niger is efficiently secreted by an endogenous $A$. niger transporter. The expression of $m f s A$ in combination with cadA led to increased citrate production suggesting that MfsA is a transporter that is able to secrete citric acid as well as itaconic acid.

Our results show that in addition to the cadA gene, the $m t t A$ gene from $A$. terreus is also crucial for efficient itaconic acid production in $A$. niger. Expression of the $m t t A$ gene, encoding a putative mitochondrial transporter, in the strain that expresses CadA resulted in an over twenty-fold increased secretion of itaconic acid. Expression of the $A$. terreus itaconic acid cluster, consisting of the cadA, $m t t A$ and $m f s A$ genes, led to $A$. niger strains with over twenty five-fold higher levels of itaconic acid and a 20-fold increase in yield when compared to a strain that expressed only CadA.

\section{Methods}

\section{Strains and spore preparations}

The fungal strains used in this study were Aspergillus terreus NRRL 1960 (Centraal Bureau voor Schimmelcultures, Baarn, The Netherlands, CBS 116.46) and Aspergillus niger NW186 (cspA1, goxC17, prtF28 $\triangle \operatorname{argB}$, pyrA6), which is a pyrA mutant of Aspergillus niger NW185 [11].

To obtain spores, 20 spores per $\mathrm{mm}^{2}$ were plated onto complete medium plates ${ }^{18}$, incubated for 5 days at $30^{\circ} \mathrm{C}$ and allowed to mature at $4^{\circ} \mathrm{C}$ for $24 \mathrm{~h}$.

The spores were harvested in $0.9 \%(\mathrm{w} / \mathrm{v}) \mathrm{NaCl}$ and $0.005 \%(\mathrm{v} / \mathrm{v})$ Tween-80, washed with $0.9 \%(\mathrm{w} / \mathrm{v}) \mathrm{NaCl}$ and stored at $4^{\circ} \mathrm{C}$ until use. 


\section{Fermentation and induction of itaconic acid production in A. terreus NRRL 1960}

A. terreus was grown at $30^{\circ} \mathrm{C}$ and $200 \mathrm{rpm}$ by inoculating spores $\left(10^{6}\right.$ spores per $\left.\mathrm{mL}\right)$ in 100 $\mathrm{mL}$ pre-cultures in $1 \mathrm{~L}$ flasks containing $25 \mathrm{~g} / \mathrm{L}$ glucose, $4.5 \mathrm{~g} / \mathrm{L} \mathrm{MgSO}{ }_{4} \cdot 7 \mathrm{H}_{2} 0,0.4 \mathrm{~g} / \mathrm{L} \mathrm{NaCl}$, $4 \mathrm{mg} / \mathrm{L} \mathrm{ZnSO}{ }_{4} \cdot 7 \mathrm{H}_{2} \mathrm{O}, 100 \mathrm{mg} / \mathrm{L} \mathrm{KH}_{2} \mathrm{PO}_{4}, 2 \mathrm{~g} / \mathrm{L} \mathrm{NH}_{4} \mathrm{NO}_{3}$ and $0.5 \mathrm{~g} / \mathrm{L}$ Corn Steep Liquor (CSL). After two days, a $10 \%(\mathrm{w} / \mathrm{v})$ inoculum was transferred to the CAD production medium, as described by Cros and Schneider ${ }^{19}$ with the following changes: $3 \mathrm{~g} / \mathrm{L} \mathrm{NH}_{4} \mathrm{NO}_{3}$ instead of urea and $1.5 \mathrm{~g} / \mathrm{L} \mathrm{MgSO}_{4} \cdot 7 \mathrm{H}_{2} \mathrm{O}$ with a final $\mathrm{pH}$ of 2.0 .

\section{Gene cloning and plasmid design}

Standard methods were used to carry out DNA manipulations and E. coli transformations. ${ }^{20}$ The gene encoding CadA was obtained by PCR from the A. terreus genome and cloned in a pUC19-derived vector under the control of the A. niger pkiA promoter ${ }^{17}$ and the terminator of the $\operatorname{trp} C$ gene of $A$. niger. The codon-optimized cadA gene was synthesized by Geneart (Invitrogen, Carlsbad, CA, US) and cloned in pUC19. Codon-optimized genes $m t t A$ and $m f s A$ were synthesized by DNA 2.0 (Menlo Park, USA) and cloned in a pUC19-derived vector under the control of a modified $x \ln D$ promoter and the terminator of the $x \ln D$ gene of $A$. niger. 13 The promoter modification involved the inactivation of CreA sites leaving the promoter inducible by D-xylose. For the construction of plasmid pLS001 the [p_x InD* - MTT - t_xInD] fragment was obtained by PCR using pMTT as a template and the following primers: LS_p_ $x \mid n D$ HindIII_for (5'-GAG-AAA-GCT-TCG-AAT-GAG-GAG-GTG-TTG-CAG $3^{\prime}$ ) and LS_t_xInD_ Xbal_rev (5'-GAG-ATC-TAG-ACT-GCA-GTC-GCA-CTC-CCG-ACC 3'). This fragment was cloned into pMFS and digested with HindIII and Xbal.

The plasmids were propagated in $\mathrm{DH} 5 \alpha$ E. coli, in LB medium (10 g/L Bacto tryptone, 5 $\mathrm{g} / \mathrm{L}$ Yeast extract, $10 \mathrm{~g} / \mathrm{L} \mathrm{NaCl}$ ) supplemented with the appropriate antibiotics $(100 \mathrm{mg} / \mathrm{L}$ ampicillin, $50 \mathrm{mg} / \mathrm{L}$ kanamycin).

\section{Fungal transformation}

For transformation of $A$. niger, protoplasts were generated using Novozyme 234. The cadA, $m t t A$ and $m f s A$ genes were introduced in $A$. niger NW186 by co-transformation as previously described ${ }^{21}$ using the pGW635 plasmid, which contains the pyrA gene ${ }^{22}$ as a primary selection marker. The pLS001 plasmid was introduced in the A. niger strain containing the cadA gene by co-transformation using the pAL69 plasmid, which contains the $\arg B$ gene as a selection marker. Selective MMS plates $\left(6.0 \mathrm{~g} / \mathrm{L} \mathrm{NaNO}_{3}, 1.5 \mathrm{~g} / \mathrm{L} \mathrm{KH}_{2} \mathrm{PO}_{4}, 0.5 \mathrm{~g} / \mathrm{L} \mathrm{KCl}, 0.5\right.$ $\mathrm{g} / \mathrm{L} \mathrm{MgSO} \mathrm{M}_{4} \cdot 7 \mathrm{H}_{2} \mathrm{O}, 1 \mathrm{~mL} / \mathrm{L}$ Vishniac, $325.2 \mathrm{~g} / \mathrm{L}$ sucrose, $1.2 \%$ (w/v) agar, pH 6.0) were used to select for protoplasts that did take up the selection marker plasmid and possibly the plasmid of interest. Randomly, 20 colonies were picked from the transformation plates and replated on complete medium. ${ }^{18}$

\section{DNA extraction and PCR of $c a d A+m t t A$ and $c a d A+m f s A$ from $A$. niger transformants}

DNA was extracted by adding $100 \mu \mathrm{L}$ extraction buffer (100 mM Tris pH 8.0, 50 mM EDTA, 
$500 \mathrm{mM} \mathrm{NaCl}, 0.07 \% \beta$-mercaptoethanol (v/v)) to the freshly harvested mycelium. The suspension was ground for 1 min using the VWR pellet mixer. $7 \mu \mathrm{L} 20 \%(\mathrm{v} / \mathrm{v})$ SDS and $26 \mu \mathrm{L}$ $5 \mathrm{M} \mathrm{KAc}$ were added and the suspension was ground again for $1 \mathrm{~min}$. The extraction samples were incubated for $10-60$ minutes at $65^{\circ} \mathrm{C}$ followed by 10 minutes on ice. The samples were centrifuged for 10 minutes at $4^{\circ} \mathrm{C}$ at $16400 \mathrm{rpm}$ using an Eppendorf Centrifuge 5417R, and the clear supernatant was transferred to a new tube. The centrifugation and transfer of supernatant was repeated. $128 \mu \mathrm{L}$ ice-cold isopropanol and $12 \mu \mathrm{L} 3 \mathrm{M} \mathrm{NaAc}$ were added to the samples, which were then incubated at $-20^{\circ} \mathrm{C}$ for 10 minutes or longer. The samples were centrifuged for 5 minutes at $4^{\circ} \mathrm{C}$ at the maximal speed and the supernatant was discarded. The pellet was washed with $70 \%$ cold ethanol, air-dried and resuspended in $\mathrm{MQ}$ water to $100 \mu \mathrm{g} \mathrm{mL}$.

To identify transformants that had integrated the $m t t A$ or $m f s A$ gene, a PCR was carried out on the extracted DNA using primers specific for the mttA gene (Fw 5'CCC-GCA-AGTACA-GTA-AGA-ACG 3' and Rv 5'CCT-GTA-CGG-AAC-CAG-ACT-CC $3^{\prime}$ ) and the $m f s A$ gene (Fw 5' TGA-TGG-GCT-CCT-TTA-ACT-GC 3' and Rv 5' GAT-AAG-ACC-GGC-GAT-AGT-GG 3').

\section{DNA extraction and PCR of $c a d A+m t t A+m f s A$ A. niger transformants}

DNA extraction and PCR was carried out to identify the colonies that randomly integrated the genes of interest. Fresh mycelium was disrupted using Fastprep and $400 \mu \mathrm{L}$ extraction buffer (100 mM TrisHCl pH 8.0, 5 mM EDTA, 1.2 M NaCl). DNA was extracted using phenolchloroform, and the pellet was washed with $70 \%$ cold ethanol, air-dried and resuspended in $50 \mu \mathrm{L} M Q$ water.

The identification of the transformants was carried out on the extracted DNA using PCR with Taq polymerase and the LS_mttA_for (5'-ATT-AAG-ACC-CGC-ATG-CAA-TC 3') and LS_mttA_ $\operatorname{rev}\left(5^{\prime}\right.$ - CTT-CTC-GTA-GAC-GGG-GAA-CA $\left.3^{\prime}\right)$ primers to check for the presence of the $m t t A$ gene. The LS_mfsA_for (5'-ACC-TTC-ACT-AGC-TGG-CGT-GT 3') and LS_mfsA_rev (5'-GACATC-CGT-GGG-ACT-GAA-CT $3^{\prime}$ ) primers were used to check for the presence of the $m f s A$ gene.

\section{Growth experiments of transformants in shake flasks}

All positively identified cadA + mttA and $c a d A+m f s A$ transformants were grown at $30^{\circ} \mathrm{C}$ and

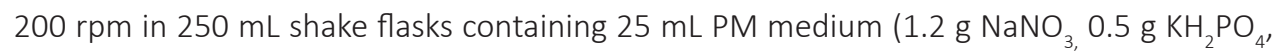
$0.2 \mathrm{~g} \mathrm{MgSO}_{4} \cdot 7 \mathrm{H}_{2} \mathrm{O}, 0.5 \mathrm{~g}$ yeast extract and $40 \mu \mathrm{L}$ Vishiniac per liter with $100 \mathrm{mM}$ sorbitol as a carbon source) ${ }^{11}$. Induction at t=0 with $10 \mathrm{mM}$ D-xylose was carried out 18 hours after inoculation. HPLC analysis was carried out on the samples after 30 hours.

Transformants containing the cadA, $m t t A$ and $m f s A$ genes were grown in $1 \mathrm{~L}$ shake flasks containing $200 \mathrm{~mL}$ PM medium as described above. Samples were taken at 6, 30, 54 and 78 $\mathrm{h}$ after induction. 


\section{Fermentation studies}

The transformants containing the $c a d A, m t t A$ and $m f s A$ genes and the control strains, sCAD4, $c a d A+m t t A 1.2$ and $c a d A+m f s A 2.5$, were inoculated $\left(10^{6}\right.$ spores $\left./ \mathrm{mL}\right)$ in $1 \mathrm{~L}$ fermenters (Sartorius) containing $0.75 \mathrm{~L}$ of PM medium with $100 \mathrm{mM}$ sorbitol. After 18 hours of growth at $30^{\circ} \mathrm{C}$, the strains were induced by the addition of $50 \mathrm{mM}$ xylose. The strains were further grown for 5 days at $30^{\circ} \mathrm{C}$ at a stirrer speed of $1000 \mathrm{rpm}$. The $\mathrm{pH}$ in the culture broth was not controlled. Samples were taken at 6, 30, 54 and 78 hours after induction.

\section{Dry weight measurement}

To determine dry weight, $10 \mathrm{~mL}$ fermentation broth was sampled and filtered using a 5 micron nylon gauze. The biomass was washed with demineralized water and completely dried on pre-weighted aluminum trays in an oven at $120^{\circ} \mathrm{C}$ for 24 hours.

\section{HPLC analysis}

High-performance liquid chromatography (HPLC) was used to determine the extracellular concentrations of sorbitol, xylose, itaconic acid, citric acid, cis-aconitic acid, pyruvic acid, $\alpha$-ketoglutaric acid, lactic acid, succinic acid, fumaric acid and oxalic acid in the samples. For organic acid measurements, a Shodex KC811 column was used and eluted with $0.01 \mathrm{~N} \mathrm{H}_{2} \mathrm{SO}_{4}$ at a flow rate of $0.8 \mathrm{~mL} \mathrm{~min}{ }^{-1}$ and sampling was carried out at $30^{\circ} \mathrm{C}$ for $25 \mathrm{~min}$. Detection was carried out using both a refractive index detector (Spectra system RI-150, sample frequency $5.00032 \mathrm{~Hz}$ ) and a UV-VIS detector (Spectrasystem UV1000, $\lambda=210 \mathrm{~nm}$ ). $6 \mathrm{mM}$ crotonate was used as an internal standard. The sugars were measured using a Dionex Carbopac MA-1 column.

\section{Determination of copy number}

DNA was extracted using the method described in "DNA extraction and PCR of $\operatorname{cadA}+m \mathrm{mtt} A$ $+m f s A$ transformants". The copy number of the cadA genes in the transformants expressing cadA from $A$. terreus or the synthetic codon-optimized cadA gene was determined in triplicate using a Rotor-Gene Q Cycler. The reaction mixture contained $8 \mu \mathrm{L} 2 \mathrm{x}$ Absolute QPCR SYBR Green mix (Thermo Scientific), $100 \mathrm{nM}$ forward and reverse primers and $2 \mu \mathrm{L}$ 100 times diluted gDNA. Primers LS_qcadA_F (5'-GAGATCTTATGGCGGTTTCCTC-3') and LS_ qcadA_R (5'-CAAGAGCTCGGGGTATCTCC-3') were used to determine the copy number of the A. terreus cadA gene and the primers LS_qcadAs_F (5'-ACTCCGAAGAGTTCGACCAG-3') and LS_qcadAs_R (5'-ACCAGGTCCTCGATTTCCTT-3') were used to determine the copy number of the synthetic cadA gene. The pkiA gene, of which only one copy is present, was used as a reference gene using the primers LS_qpkiA_F (5'-GGTAAC GACAGCGATTGGAT-3') and LS_ qpkiA_R (5'-GGGCTCAAAGTGAATGTGGT-3'). Water and SDS samples were used as controls. The qPCR cycling program was as follows: $15 \mathrm{~min}$ initial polymerase activation at $95^{\circ} \mathrm{C}$ followed by $40 \mathrm{cycles}$ of $95^{\circ} \mathrm{C}$ for $15 \mathrm{sec}, 59^{\circ} \mathrm{C}$ for $15 \mathrm{sec}$ and $72^{\circ} \mathrm{C}$ for $30 \mathrm{sec}$. The calculations were carried out using the Pfaffl method. ${ }^{16}$ 


\section{Competing interests}

The authors declare that they have no competing interests.

\section{Acknowledgements}

The authors gratefully acknowledge Ruud Heshof for providing the modified $x \ln D$ promoter. This work has been carried out on the basis of a grant in the framework of the BE BASIC program F01.002 Itaconic/fumaric acids: Novel economic and eco-efficient processes for the production of itaconic and fumaric acid. 


\section{References}

1. Kubicek CP, Punt PJ, Visser J. Production of organic acids by filamentous fungi. The Mycota. Industrial Applications, Edited by M. Hofrichter, Berlin, Heidelberg: Springer Berlin Heidelberg. 2010:215-234

2. Okabe M, Lies D, Kanamasa S, Park EY. Biotechnological production of itaconic acid and its biosynthesis in Aspergillus terreus. Appl Microbiol Biotechnol 2009,84:597-606.

3. Ferraboschi P, Casati S, Grisenti P. Selective enzymatic transformations of itaconic acid derivatives: An access to potentially useful building blocks. Tetrahedron 1994,50:3251-3258.

4. Werpy T, Petersen G. Top Value Added Chemicals from Biomass: Volume I. Results of screening for potential candidates from sugars and synthesis gas. Pacific Northwest National Laboratory, National Renewable Energy Laboratory; 2004:1-76.

5. Willke T, Vorlop K-D. Biotechnological production of itaconic acid. Appl Microbiol Biotechnol 2001,56:289-295.

6. Magnuson J, Lasure L. Organic acid production by filamentous fungi. In Advances in Fungal Biotechnology for Industry, Agriculture and Medicine. Edited by Tkacz JS, Lange L. New York: Kluwer Academic/Plenum Publishers; 2004:307-340.

7. Kanamasa S, Dwiarti L, Okabe M, Park EY. Cloning and functional characterization of the cis-aconitic acid decarboxylase (CAD) gene from Aspergillus terreus. Appl Microbiol Biotechnol 2008,80:223229.

8. Li A, van Luijk N, Beek Ter M, Caspers M, Punt P, der Werf van M. A clone-based transcriptomics approach for the identification of genes relevant for itaconic acid production in Aspergillus. Fungal Genet Biol 2011,48:602-611.

9. Kennedy J, Auclair K, Kendrew SG, Park C, Vederas JC, Hutchinson CR. Modulation of polyketide synthase activity by accessory proteins during lovastatin biosynthesis. Science 1999,284:13681372.

10. Li A, Pfelzer N, Zuijderwijk R, Brickwedde A, van Zeijl C, Punt P. Reduced by-product formation and modified oxygen availability improve itaconic acid production in Aspergillus niger. Appl Microbiol Biotechnol 2013,9:3901-3911.

11. Ruijter GJ, de Vondervoort van PJ, Visser J. Oxalic acid production by Aspergillus niger: an oxalatenon-producing mutant produces citric acid at $\mathrm{pH} 5$ and in the presence of manganese. Microbiology 1999,145:2569-2576.

12. der Veen van D, Oliveira JM, den Berg van WAM, de Graaff LH. Analysis of variance components reveals the contribution of sample processing to transcript variation. Appl Environ Microbiol 2009,75:2414-2422.

13. van Peij NN, Brinkmann J, Vrsanská M, Visser J, de Graaff LH. beta-Xylosidase activity, encoded by $x \ln D$, is essential for complete hydrolysis of xylan by Aspergillus niger but not for induction of the xylanolytic enzyme spectrum. Eur J Biochem 1997,245:164-173.

14. Koivistoinen OM, Richard P, Penttilä M, Ruohonen L, Mojzita D. Sorbitol dehydrogenase of Aspergillus niger, SdhA, is part of the oxido-reductive D-galactose pathway and essential for D-sorbitol catabolism. FEBS Lett 2012,586:378-383. 
15. The Aspergillus Website [http://www.aspergillus.org.uk]

16. Pfaffl, MW. A new mathematical model for relative quantification in real-time RT-PCR. Nucleic Acids Res 2001,29:2002-2007.

17. de Graaff L, den Broeck van H, Visser J. Isolation and characterization of the Aspergillus niger pyruvate kinase gene. Curr Genet 1992,22:21-27.

18. Pontecorvo G, Roper JA, Hemmons LM, MacDonald KD, Bufton AWJ. The genetics of Aspergillus nidulans. Adv Genet 1953,5:141-238.

19. Cros P, Schneider D. Patent US5231016 - Microbiological production of itaconic acid. 1993.

20. Sambrook JF, Fritsch EF, Maniatis T. Molecular Cloning: A Laboratory Manual. Cold Spring Harbor, NY: Cold Spring Harbor Laboratory; 1989.

21. Kusters-van Someren MA, Harmsen JA, Kester HC, Visser J. Structure of the Aspergillus niger pelA gene and its expression in Aspergillus niger and Aspergillus nidulans. Curr Genet 1991,20:293-299.

22. Goosen T, Bloemheuvel G, Gysler C, Bie DA, Broek HWJ, Swart K. Transformation of Aspergillus niger using the homologous orotidine-5?-phosphate-decarboxylase gene. Curr Genet 1987,11:499-503. 



\section{6}

(9)
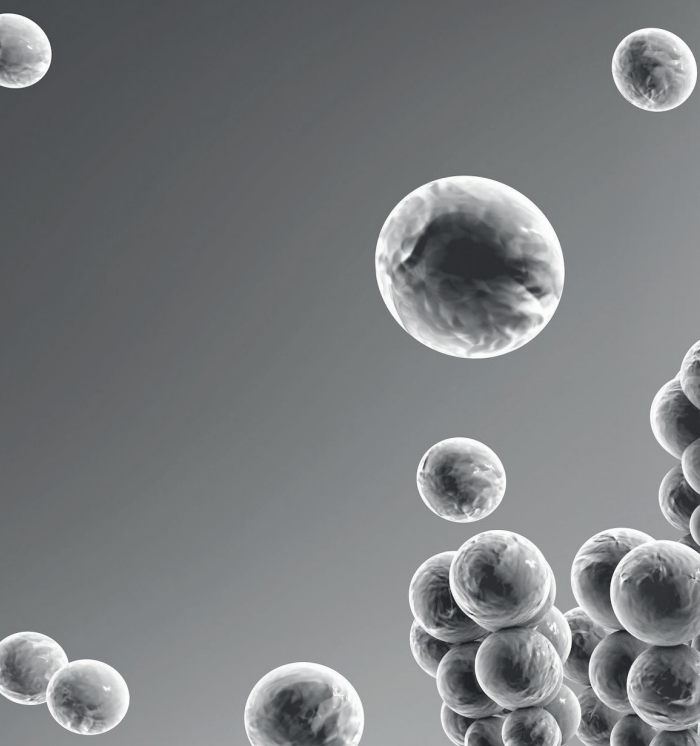

6

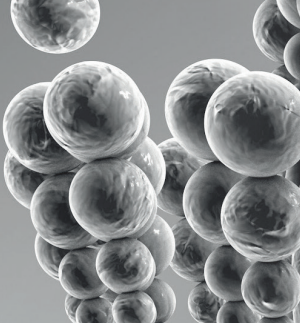

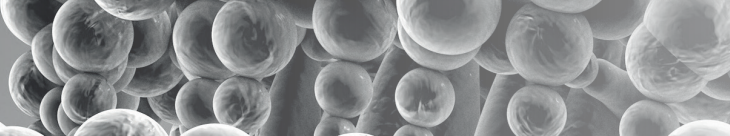
(1)
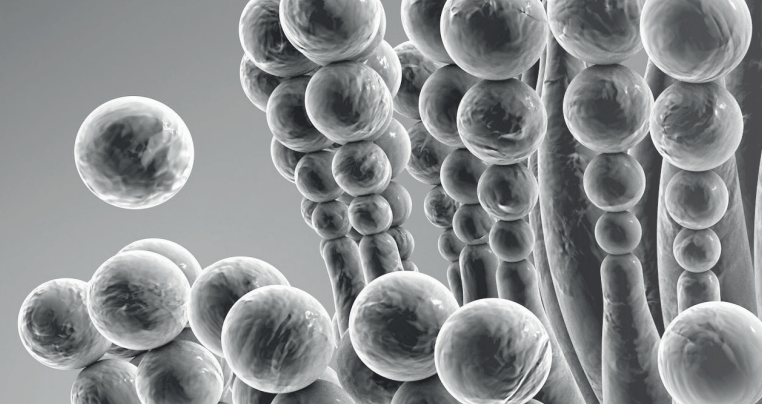

(9)
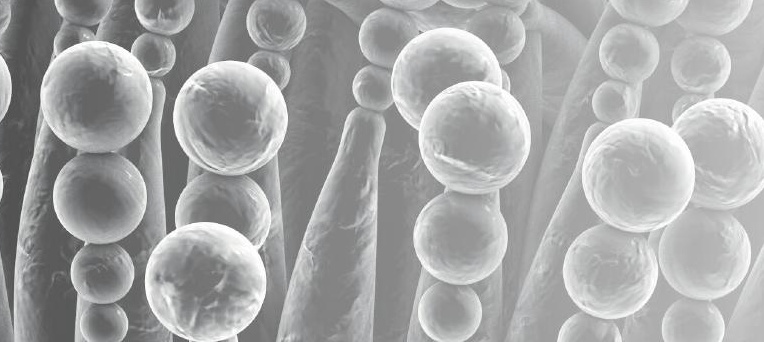

$$
\text { Q }
$$
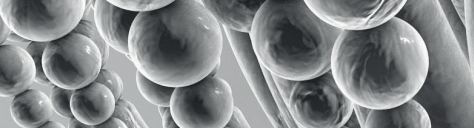

428

init 180

il mo

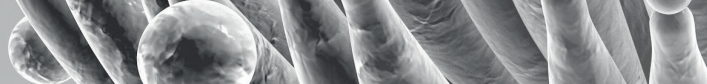

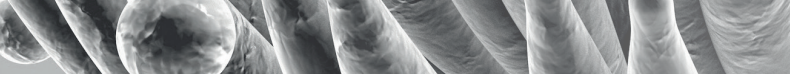

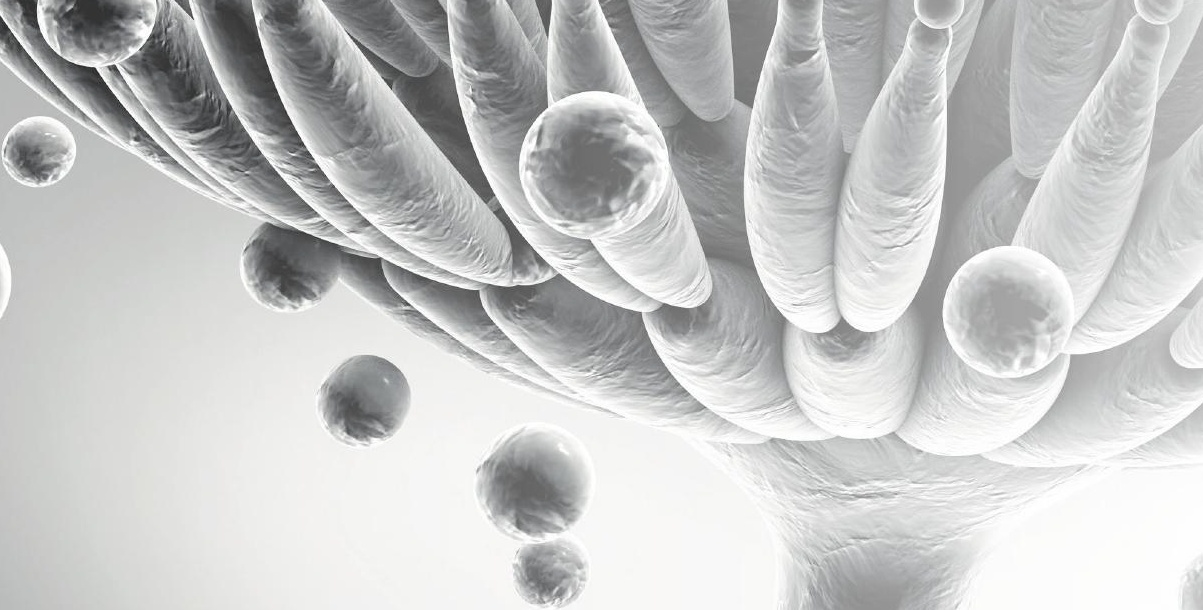

(.

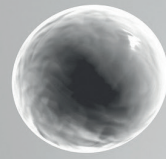

6 


\section{Chapter 3}

\section{Pathway transfer in filamentous fungi: transporters are the key to success}




\section{Abstract}

Itaconic acid is an important building block for the chemical industry. Currently, Aspergillus terreus is the main organism used for itaconic acid production. Due to the enormous citric acid production capacity of Aspergillus niger, this host is investigated as a potential itaconic acid production host. Several strategies have been tried so far; fermentation optimization, expression of cis-aconitate decarboxylase ( $\operatorname{cadA}$ ) alone and in combination with aconitase targeted to the same compartment, chassis optimization and the heterologous expression of two transporters flanking the cadA gene. We showed that the heterologous expression of these transporters were key to improving itaconic acid production in an A. niger strain that was unable to produce oxalic acid and gluconic acid. The expression of transporters has increased the production levels of other industrially relevant processes as well, such as beta-lactam antibiotics and bioethanol. Thus far, the role of transporters in production process optimization is a bit overlooked. 


\section{Introduction}

Over the past few years there is an increasing interest in itaconic acid as a biobased building block to produce e.g. plastics, adhesives, elastomers and coatings. Besides, itaconic acid is also used as a building block for materials used for biomedical purposes like drug delivery systems and as a material in restorative dentistry. ${ }^{1}$ In search of optimized production processes for the production of itaconic acid from plant waste streams, Aspergillus niger is investigated as a cell factory by several research groups.

A. niger is one of the organisms of choice to exploit plant derived waste streams due to its extensive capacity to degrade polysaccharides. ${ }^{2}$ In addition, A. niger has a long history in industrial fermentations and can be easily genetically modified making the organism an attractive host for the production of enzymes and metabolites.

Itaconic acid is nowadays produced on an industrial scale using the filamentous fungus Aspergillus terreus. Titers up to $80 \mathrm{~g} / \mathrm{L}$ are achieved and several strategies have been investigated to further improve the production in this strain.

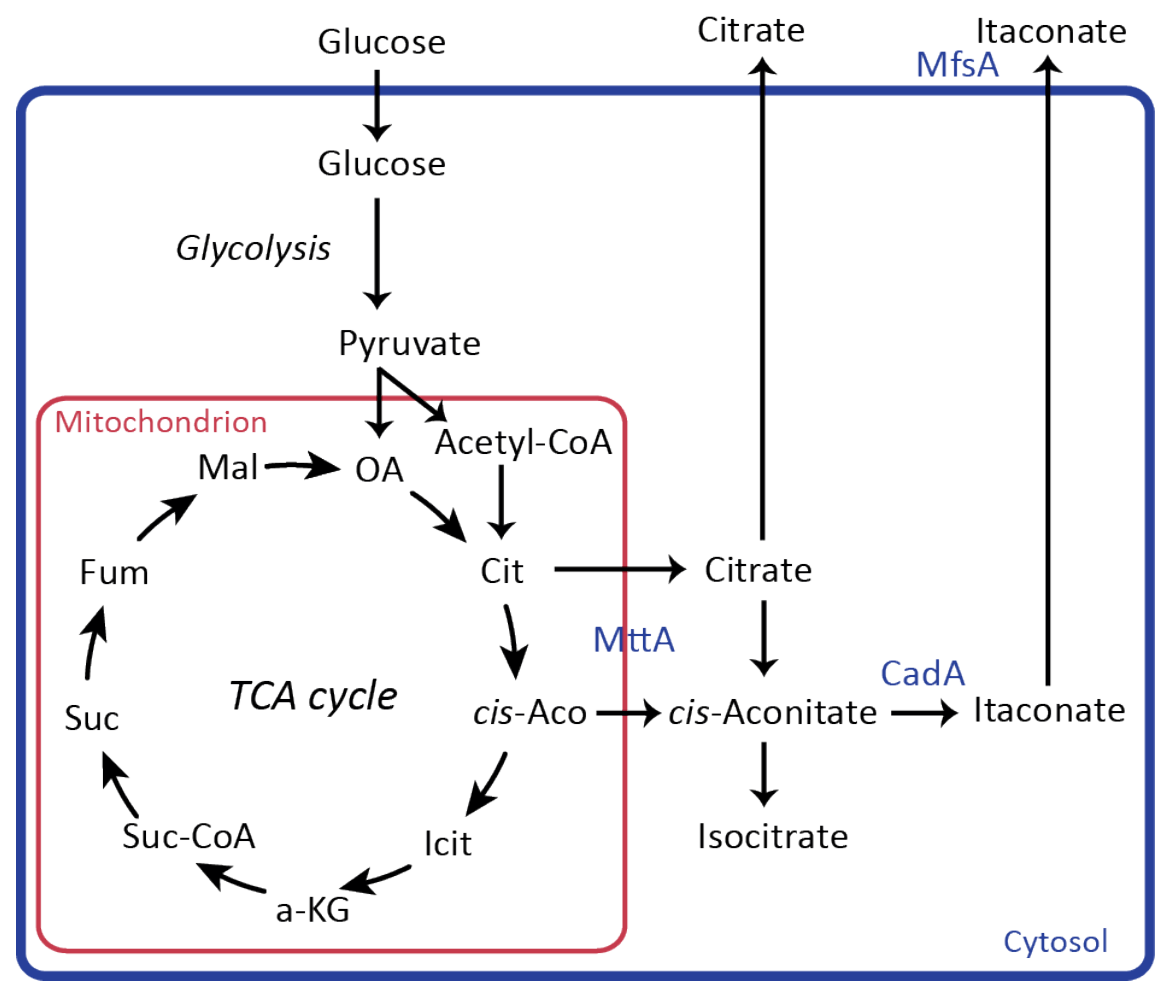

Figure 3.1 Schematic representation of the citric acid biosynthesis pathway in Aspergillus niger extended with the itaconic acid biosynthesis pathway. The transport of cis-aconitate from the mitochondria to the cytosol is speculative. 
Itaconic acid biosynthesis is very similar to citric acid biosynthesis because it is basically an extension of the citric acid biosynthesis pathway (Fig. 3.1). Aconitase catalyzes the isomerization of citrate to isocitrate via cis-aconitate. The decarboxylation of the intermediate cis-aconitate by cis-aconitate decarboxylase leads to the formation of itaconate.

A. niger has been exploited for citric acid production for many years and extremely high citrate production levels, over $200 \mathrm{~g} / \mathrm{L}$, are achieved nowadays. ${ }^{3}$ If these citrate production strains can be modified for itaconic acid production, titers over $113 \mathrm{~g} / \mathrm{L}$ could be reached.

\section{Itaconic acid production in Aspergillus niger}

Oxalic acid is the major organic acid being produced by $A$. niger laboratory strains. Besides oxalic acid also citric acid and gluconic acid are produced in large amounts. The pathway transfer of itaconic acid production to $A$. niger started with the overexpression of the $A$. terreus cadA gene encoding cis-aconitate decarboxylase in $A$. niger. This led to the production of low amounts of itaconic acid. ${ }^{4,5}$

These initial results showed that the production of itaconic acid in A. niger is possible, but the production level obtained is far from the theoretical levels if only cadA is expressed. To improve the itaconic acid production several strategies have been applied. These strategies target different levels of the production process.

\section{Fermentation optimization}

One of the strategies applied is to optimize the fermentation conditions and in particular the medium composition. Though positive and negative effects of several components were found, the most pronounced positive effect was the addition of copper, although the exact function of copper on organic acid production is still unknown. By optimizing the copper concentration the oxalic acid production was reduced, while the citric acid production and in parallel the itaconic acid production increased. Nevertheless, even after medium optimization, the itaconic acid concentration obtained was only $2 \mathrm{~g} / \mathrm{L}$. ${ }^{6}$

\section{Chassis optimization}

An early example of host or chassis optimization for citric acid production is the work of Ruijter et al. (1999). With the aim to reduce side product formation in citric acid production and to increase the carbon flow towards citric acid production, these authors constructed strains deficient in oxalic acid and gluconic acid biosynthesis. Loss-of-function mutations in oxaloacetatehydrolase (OahA) and glucose oxidase (GoxC) results in the inability to produce any oxalic acid and gluconic acid. Surprisingly, combining these mutations in $A$. niger results in constitutive citric acid production, which is then no longer influenced by the carbon source or medium composition. ${ }^{7}$ 
Li et al. showed the importance of chassis optimization in side product formation in itaconic acid production by $A$. niger through expressing cadA in an oahA $A$. niger strain. This resulted in $0.4 \mathrm{~g} / \mathrm{L}$ itaconic acid, which was also an improvement compared the $0.24 \mathrm{~g} / \mathrm{L}$ that was obtained by the expression of cadA in a wild type background. ${ }^{8}$ Our studies on the production of itaconic acid in $A$. niger were performed in a genetic background lacking both OahA and GoxC activity resulting in itaconic acid production levels that are far above the levels thus far described. ${ }^{9}$

\section{Pathway optimization}

An elegant way to improve itaconic acid production was shown by Blumhoff et al. They targeted the crucial enzymes, aconitase and CadA, to the same compartment. It is believed that CadA is localised in the cytosol while aconitase activity is mainly found in the mitochondria as part of the TCA cycle (Fig. 3.1). ${ }^{10}$ The itaconic acid production level increased significantly when both enzymes were targeted to the mitochondria. ${ }^{11}$ This indicates that the substrate limitation might actually be a transport limitation between the different compartments.

Another approach was the combined overexpression of a modified phosphofructokinase in combination with the itaconic acid biosynthesis cluster. Using phosphofructokinase that was relieved of citrate inhibition led to increased productivity levels but not to improved production levels. ${ }^{12}$

\section{Optimization of cellular transport}

In a recent paper we showed that besides the overexpression of CadA and the chassis optimization, two transporters play a crucial role in establishing an efficient production process. Especially the heterologous expression of the mitochondrial transporter improved the production significantly in this particular A. niger strain. ${ }^{9}$

An overview of the most interesting modifications to establish and optimize itaconic acid production in A. niger is given in Table 3.1. 
Table 3.1 Overview of the constructed itaconic acid producing Aspergillus niger strains

\begin{tabular}{|c|c|c|c|c|}
\hline Strain & $\begin{array}{l}\text { A. niger } \\
\text { parent } \\
\text { strain }\end{array}$ & Expressed genes & $\begin{array}{l}\text { Itaconic } \\
\text { acid } \\
\text { yield } \\
\text { in } \mathrm{g} / \mathrm{L}\end{array}$ & Growth conditions \\
\hline $\mathrm{sCAD}^{9}{ }^{9}$ & NW186 & $\operatorname{cadA}$ & 0.25 & $\begin{array}{l}100 \text { mM sorbitol }+50 \\
\text { mM xylose, } 78 \text { hours of } \\
\text { fermentation }\end{array}$ \\
\hline$c a d A+m f s A 2.5^{9}$ & sCAD4 & $c a d A+m f s A$ & 0.14 & $\begin{array}{l}100 \text { mM sorbitol + } 50 \\
\text { mM xylose, } 78 \text { hours of } \\
\text { fermentation }\end{array}$ \\
\hline $\operatorname{cad} A+m t t A 1.2^{9}$ & sCAD4 & $c a d A+m t t A$ & 5.4 & $\begin{array}{l}100 \text { mM sorbitol }+50 \\
\text { mM xylose, } 78 \text { hours of } \\
\text { fermentation }\end{array}$ \\
\hline$c a d A+m t t A+m f s A 4^{9}$ & sCAD4 & $c a d A+m t t A+m f s A$ & 7.1 & $\begin{array}{l}100 \text { mM sorbitol }+50 \\
\text { mM xylose, } 78 \text { hours of } \\
\text { fermentation }\end{array}$ \\
\hline CAD10.1 $1^{4}$ & AB 1.13 & $\operatorname{cad} A$ & 0.7 & $\begin{array}{l}100 \mathrm{~g} / \mathrm{L} \text { glucose after } 90 \text { hours } \\
\text { of fermentation }\end{array}$ \\
\hline CAD $10.1^{8}$ & AB 1.13 & $\operatorname{cadA}$ & 0.24 & Micro-titer cultures, 60 hours \\
\hline$\triangle$ oahA\#76 CAD 5 & $\begin{array}{l}\text { AB } 1.13 \\
\text { DoahA\#76 }\end{array}$ & $\operatorname{cad} A$ & 0.4 & Micro-titer cultures, 60 hours \\
\hline MTT $1.4^{8}$ & CAD10.1 & $\operatorname{cad} A+m t t A$ & 1.4 & Micro-titer cultures, 60 hours \\
\hline MFS $3.9^{8}$ & CAD10.1 & $\operatorname{cad} A+m f s A$ & 1.4 & Micro-titer cultures, 60 hours \\
\hline$C A D+M T T+M F S \_3^{8}$ & MTT 1.4 & $c a d A+m t t A+m f s A$ & 0.9 & Micro-titer cultures, 60 hours \\
\hline cCadA $^{11}$ & ATCC 1015 & cytosolic cadA & 0.05 & $\begin{array}{l}\text { Shakeflask culture with } 20 \% \\
\text { glucose for } 240-312 \text { hours }\end{array}$ \\
\hline $\mathrm{mCadA}^{11}$ & ATCC 1015 & mitochondrial cadA & 0.17 & $\begin{array}{l}\text { Shakeflask culture with } 20 \% \\
\text { glucose for } 240-312 \text { hours }\end{array}$ \\
\hline$m C a d A+m A c n A^{11}$ & ATCC 1015 & $\begin{array}{l}\text { mitochondrial } \operatorname{cad} A+ \\
\text { mitochondrial } a c o A \\
\text { from } A \text {. niger }\end{array}$ & 1.2 & $\begin{array}{l}\text { Shakeflask culture with } 20 \% \\
\text { glucose for } 240-312 \text { hours }\end{array}$ \\
\hline $\begin{array}{l}c \mathrm{CadA}+\mathrm{cAcnA}+ \\
\mathrm{mCadA}+\mathrm{mAcn}^{11}\end{array}$ & ATCC 1015 & $\begin{array}{l}\text { cytosolic cadA + cytosolic } \\
\text { acnA from E. coli }+ \\
\text { mitochondrial cadA + } \\
\text { mitochondrial acoA from } \\
\text { A. niger }\end{array}$ & 1.4 & $\begin{array}{l}\text { Shakeflask culture with } 20 \% \\
\text { glucose for } 240-312 \text { hours }\end{array}$ \\
\hline
\end{tabular}




\section{Cellular transport}

Heterologous expression of transporters is rarely done as part of strain improvement, while it does have a great potential to improve production levels of industrially relevant compounds. We showed that the overexpression of the mitochondrial transporter MttA improved the itaconic acid production by a factor of 18 . The subsequent expression of the plasmamembrane transporter MfsA improved the production even further. A few more examples are found in literature where the expression of transporters improved production levels.

\section{Expression of transporters to enhance the production of antibiotics}

Penicillin production in Penicillium chrysogenum is a well-studied process due to its importance as an antibiotic. Metabolic engineering strategies dramatically improved production levels of penicillin by Penicillium species over the years. The majority of these strategies focussed on the metabolic conversions in the cell, but rarely on the transport between the different compartments. Even though the production of penicillin in $P$. chrysogenum is highly optimized over the years, it still seems that especially the export of the product is a limiting factor of the penicillin production process. ${ }^{13}$

Due to increasing penicillin resistance new beta-lactam antibiotics are produced e.g. cephalosporins and cephamycins. P. chrysogenum is optimized for the production of betalactam antibiotics and therefore serves as a chassis for the production of several other betalactam antibiotics. One example is the production of ad7-ACCCA by introducing genes from Acremonium chrysogenum and Streptomyces clavuligerus. ${ }^{14}$

Only in the last few years studies appeared on the functioning of a few important transporters in beta-lactam antibiotic production. One very interesting transporter is the cefT gene from A. chrysogenum. The heterologous expression of this transporter in different $P$. chrysogenum strains resulted in an increased secretion of the desired beta-lactam antibiotic. ${ }^{15,16}$ Part of the beta-lactam antibiotics biosynthesis takes place in the peroxisomes while the major part of the biosynthesis takes place in the cytosol. Thus besides an efficient import of the substrate and export of the product also peroxisomal transport is an important process in beta-lactam antibiotics production.

In A. chrysogenum the transporter CefM was found that is essential for the transport of penN from the peroxisomes to the cytosol. ${ }^{17}$

\section{L-Galactonic acid production by filamentous fungi}

Pectin is a major plant cell wall component in certain agricultural waste streams like sugar beet pulp. Since sugar beet pulp is mostly dumped, developing a process for the production of metabolites using sugar beet pulp is economically attractive. Pectin is easily hydrolysed by filamentous fungi to D-galacturonic acid, which is subsequently further metabolised. Kuivanen et al. describe the production of L-galactonic acid from D-galacturonic acid and 
polygalacturonic acid by Trichoderma reesei and A. niger. ${ }^{18}$ In this study two effects on cellular transport were found; increased production of a putative D-galacturonic acid transporter and intracellular accumulation of L-galactonic acid. The latter suggests a bottleneck in L-galactonic acid export that may be relieved by the introduction of an L-galactonic acid transporter. As the uptake of D-galacturonic acid might also be a bottleneck, increased expression of the D-galacturonic acid transporter may increase the efficiency of the process as well. Overexpression of a D-galacturonic acid transporter in A. niger leads to preferred use of D-galacturonic acid paralleled by increased citric acid formation (Sloothaak \& de Graaff, personal communication). This might open ways to exploit the pectin fraction of plant biomass for biobased production.

\section{Expression of a xylose transporter in Saccharomyces cerevisiae}

Saccharomyces cerevisiae is one of the most-studied organisms in biotechnology and serves as a model organism for eukaryotes. S. cerevisiae is used in industry for the production of ethanol. Currently, the production of ethanol from plant waste materials is a trending topic in biotechnology. One of the major drawbacks of wildtype S. cerevisiae is its inability to grow on xylose. After glucose, xylose is the most abundant sugar present in plant cell wall polysaccharides. Therefore, a lot of research is done on the heterologous expression of xylose metabolizing pathways in S. cerevisiae. ${ }^{19,20}$

Besides the enzymatic conversion of xylose also the import of xylose into the cell has been investigated.

The heterologous expression of xylose transporters in an S. cerevisiae strain that is able to use xylose for the production of ethanol showed that the production rate of ethanol increased up to 70\%. ${ }^{21}$ This example also shows that in this efficient ethanol production process import of xylose was a limiting step and major improvements can still be made by the expression of transporters. Recently, Young et al. showed that they were able to improve xylose transporters even further using directed evolution. Only a few point mutations already led to an improved growth rate by $70 \%$. Other mutations caused differences in efficiency and changes in affinity of the transporter. ${ }^{22}$

\section{Outlook}

The transfer of biosynthetic pathways that lead to the production of metabolites of industrial importance from one organism to a biotechnologically more preferred organism has become more feasible than ever. Pathway transfer became a realistic possibility after the development of modern cloning techniques. Nowadays, synthetic biology increases the possibilities for effective pathway transfer enormously. The use of standardized modules combined with metabolic modeling allows the rational design of a desired pathway suitable for the new host or chassis. Routinely, codon usage is optimized to achieve efficient expression of the 
coding sequence by the production host. Combining codon usage optimization with the use of well-defined and characterized parts like promoters, terminators and signal sequences will significantly decrease the time needed for the development of future processes.

However, transporters are often overlooked when transferring pathways from one organism to the other. This is not surprising since it is more difficult to identify and characterize transporters compared to enzymes. Despite the difficulties, new strategies became available to characterize transporters. A nice example is the expression of eukaryotic transporters in Lactococcus lactis as an alternative host for E. coli. ${ }^{23-25}$ A success story using L. lactis as an expression host is the characterization of the mitochondrial pyruvate transporter that is encoded by two genes. ${ }^{26}$

As the evolutionary distance between different organisms increases, differences in membrane composition and membrane structure become apparent. Even though the function of a given transporter might be known in one organism it might be a challenge to functionally express the transporter in the host of choice due to these differences.

Transporters play a crucial role in optimizing pathways for the production of industrially relevant compounds. The here-described studies on the production of itaconic acid in $A$. niger, beta-lactam antibiotics production in $P$. chrysogenum and the bioethanol production from xylose in S. cerevisae are a showcase of the knowledge that needs to be developed. Many more success stories will follow in the future when more knowledge is aquired about the function of transport proteins. Transporters might be the key to success when transfering pathways to fungi.

\section{Acknowledgements}

This work has been carried out on the basis of a grant in the framework of the BE BASIC program F01.002 Itaconic/fumaric acids: Novel economic and eco-efficient processes for the production of itaconic and fumaric acid 


\section{References}

1. Okabe M, Lies D, Kanamasa S, Park EY. Biotechnological production of itaconic acid and its biosynthesis in Aspergillus terreus. Appl Microbiol Biotechnol 2009,84:597-606.

2. van den Brink J, de Vries RP. Fungal enzyme sets for plant polysaccharide degradation. Appl Microbiol Biotechnol 2011,91:1477-92.

3. Magnuson J, Lasure L. Organic acid production by filamentous fungi. Advances in fungal biotechnology for Industry, Agriculture and Medicine 2004:307-340

4. Li A, van Luijk N, Beek ter M, Caspers M, Punt P, der Werf van M. A clone-based transcriptomics approach for the identification of genes relevant for itaconic acid production in Aspergillus. Fungal Genet Biol 2011,48:602-11.

5. Blumhoff M, Steiger MG, Marx H, Mattanovich D, Sauer M. Six novel constitutive promoters for metabolic engineering of Aspergillus niger. Appl Microbiol Biotechnol 2012, 97:259-267.

6. Li A, Pfelzer N, Zuijderwijk R, Punt P. Enhanced itaconic acid production in Aspergillus niger using genetic modification and medium optimization. BMC Biotechnol 2012,12:57.

7. Ruijter G, de Vondervoort van P. Oxalic acid production by Aspergillus niger: an oxalate-nonproducing mutant produces citric acid at pH 5 and in the presence of manganese. Microbiology 1999,145:2569-2576.

8. Li A, Pfelzer N, Zuijderwijk R, Brickwedde A, van Zeijl C, Punt P. Reduced by-product formation and modified oxygen availability improve itaconic acid production in Aspergillus niger. Appl Microbiol Biotechnol 2013,97:3901-3911.'V B

9. van der Straat L, Vernooij M, Lammers M, van den Berg W, Schonewille T, Cordewener J, van der Meer I, Koops A, de Graaff LH. Expression of the Aspergillus terreus itaconic acid biosynthesis cluster in Aspergillus niger. Microb Cell Fact 2014,13:11.

10. Jaklitsch WM, Kubicek C, Scrutton MC. The subcellular organization of itaconate biosynthesis in Aspergillus terreus. J Gen Microbiol 1991,137:533-539.

11. Blumhoff ML, Steiger MG, Mattanovich D, Sauer M. Targeting enzymes to the right compartment: Metabolic engineering for itaconic acid production by Aspergillus niger. Metab Eng 2013,19C:26-32.

12. van der Straat L, Tamayo-Ramos JA, Schonewille T, de Graaff LH. Overexpression of a modified 6-phosphofructo-1-kinase results in an increased itaconic acid productivity in Aspergillus niger. AMB Express 2013,3:57.

13. Douma RD, Deshmukh AT, de Jonge LP, de Jong BW, Seifar RM, Heijnen JJ, van Gulik WM. Novel insights in transport mechanisms and kinetics of phenylacetic acid and penicillin-G in Penicillium chrysogenum. Biotechnol Progress 2012,28:337-48.

14. Harris DM, Westerlaken I, Schipper D, van der Krogt ZA, Gombert AK, Sutherland J, Raamsdonk LM, van den Berg MA, Bovenberg RAL, Pronk JT, et al. Engineering of Penicillium chrysogenum for fermentative production of a novel carbamoylated cephem antibiotic precursor. Metab Eng 2009,11:125-37. 
15. Nijland JG, Kovalchuk A, van den Berg MA, Bovenberg RAL, Driessen AJM. Expression of the transporter encoded by the cefT gene of Acremonium chrysogenum increases cephalosporin production in Penicillium chrysogenum. Fungal Genet Biol 2008,45:1415-21.

16. Ullán RV, Teijeira F, Martín JF. Expression of the Acremonium chrysogenum cefT gene in Penicillum chrysogenum indicates that it encodes an hydrophilic beta-lactam transporter. Curr Genet 2008,54:153-161.

17. Teijeira F, Ullán RV, Guerra SM, Garcia-Estrada C, Vaca I, Martín JF. The transporter CefM involved in translocation of biosynthetic intermediates is essential for cephalosporin production. Biochem J 2009,418:113-124.

18. Kuivanen J, Mojzita D, Wang Y, Hilditch S, Penttilä M, Richard P, Wiebe MG. Engineering filamentous fungi for conversion of D-galacturonic acid to L-galactonic acid. Appl Environ Microbiol 2012,78:8676-8683.

19. Kim SR, Park Y-C, Jin Y-S, Seo J-H. Strain engineering of Saccharomyces cerevisiae for enhanced xylose metabolism. Biotechnol Adv 2013,31:851-861.

20. Hahn-Hägerdal B, Karhumaa K, Fonseca C, Spencer-Martins I, Gorwa-Grauslund MF. Towards industrial pentose-fermenting yeast strains. Appl Microbiol Biotechnol 2007, 74:937-953.

21. Hector RE, Qureshi N, Hughes SR, Cotta MA. Expression of a heterologous xylose transporter in a Saccharomyces cerevisiae strain engineered to utilize xylose improves aerobic xylose consumption. Appl Microbiol Biotechnol 2008,80:675-684.

22. Young EM, Comer AD, Huang $\mathrm{H}$, Alper HS. A molecular transporter engineering approach to improving xylose catabolism in Saccharomyces cerevisiae. Metab Eng 2012, 14:401-411.

23. Kunji E, Chan KW, Slotboom DJ, Floyd S, O'Connor R, Monne M. Eukaryotic membrane protein overproduction in Lactococcus lactis. Curr Opin Biotechnol 2005,16:546-551.

24. Kunji ERS, Slotboom D-J, Poolman B. Lactococcus lactis as host for overproduction of functional membrane proteins. Biochim Biophys Acta 2003,1610:97-108.

25. Monne M, Chan KW, Slotboom DJ, Kunji E. Functional expression of eukaryotic membrane proteins in Lactococcus lactis. Protein Sci 2005,14:3048-3056.

26. Herzig S, Raemy E, Montessuit S, Veuthey J-L, Zamboni N, Westermann B, Kunji ERS, Martinou $\mathrm{J}-\mathrm{C}$. Identification and functional expression of the mitochondrial pyruvate carrier. Science 2012,337:93-96. 


\section{6}

(9)
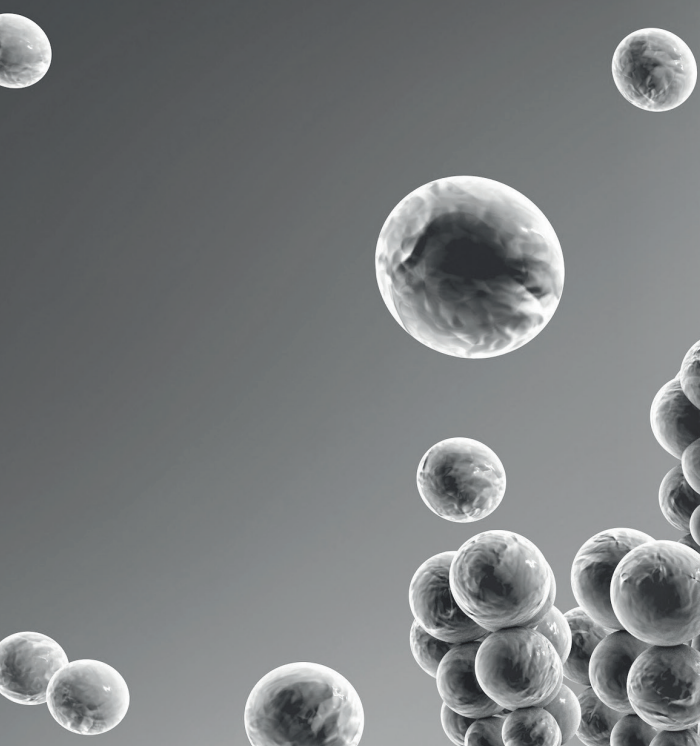

6

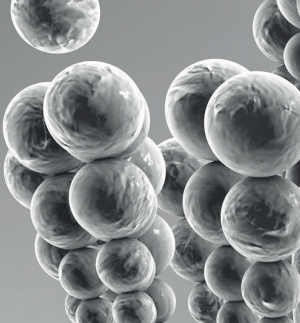

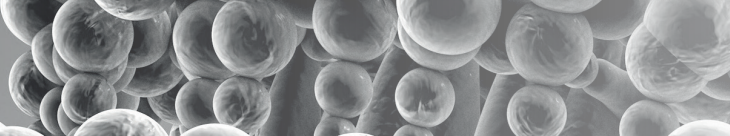
(1)
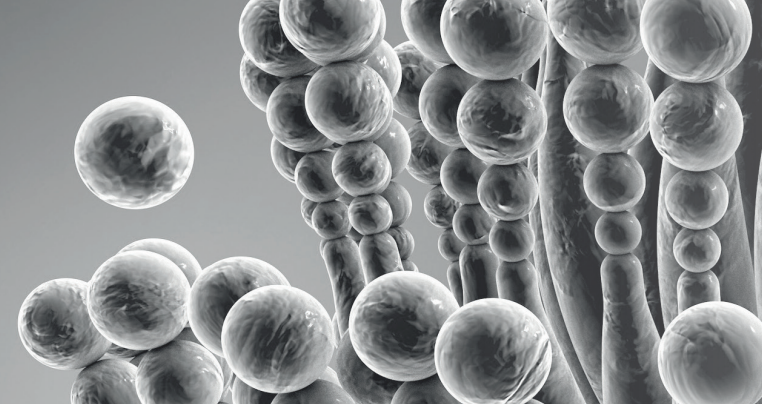

(9)
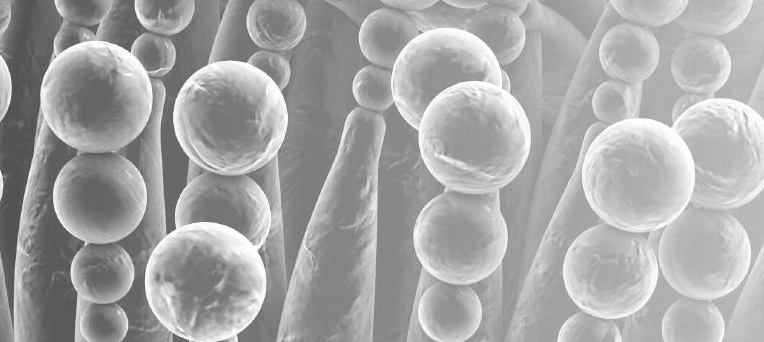

$$
\text { Q }
$$
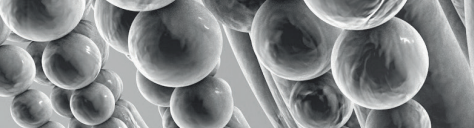

428

init 180

il mo

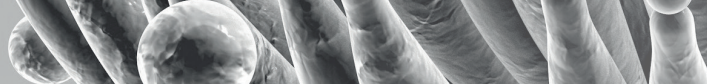

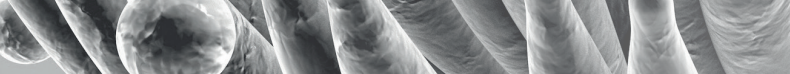

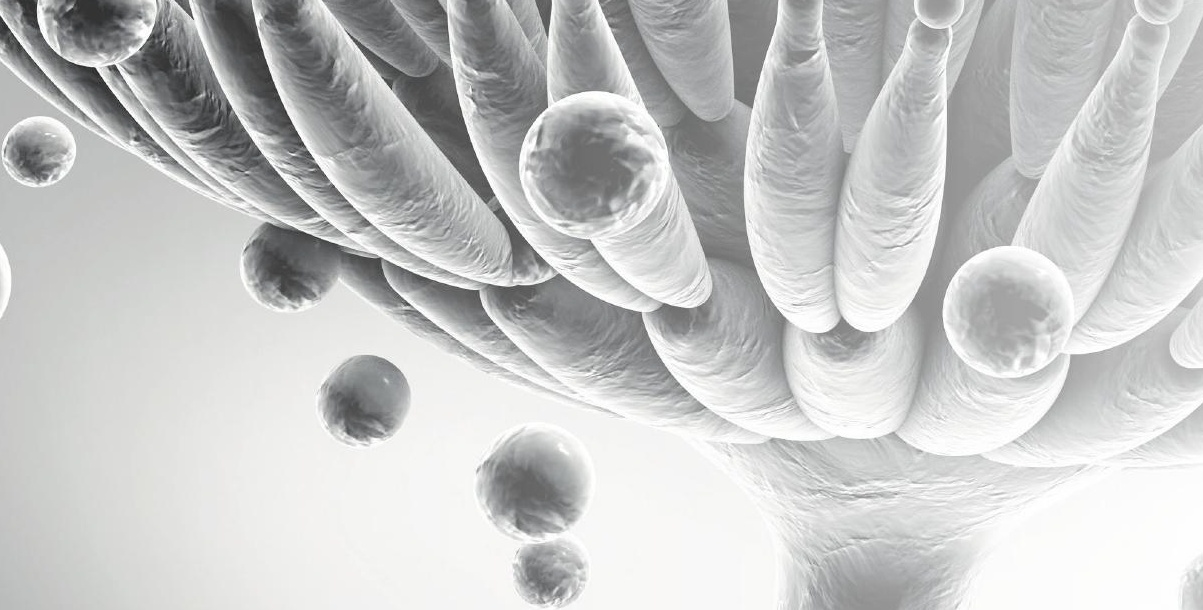

(.

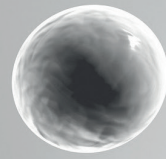

6 


\title{
Chapter 4
}

\author{
Overexpression of a modified \\ 6-phosphofructo-1-kinase results \\ in an increased itaconic acid \\ productivity in Aspergillus niger
}

L. van der Straat, J.A. Tamayo-Ramos, T. Schonewille and L.H. de Graaff. Overexpression of a modified 6-phosphofructo-1-kinase results in an increased itaconic acid productivity in Aspergillus niger. AMB Express, 2013,3:57 


\section{Abstract}

A modified 6-phosphofructo-1-kinase was expressed in a citrate producing Aspergillus niger strain in combination with cis-aconitate decarboxylase from Aspergillus terreus to study the effect on the production of itaconic acid. The modified pfkA gene was also expressed in combination with the itaconic acid biosynthetic cluster from $A$. terreus, which consists of cis-aconitate decarboxylase $c a d A$, a putative mitochondrial transporter $m t t A$ and a putative plasmamembrane transporter $m f s A$. The combined expression of $p f k A$ and $c a d A$ resulted in increased citrate levels, but did not show increased itaconic acid levels. The combined expression of $p f k A$ with the itaconic acid biosynthetic cluster resulted in significantly increased itaconic acid production at earlier time points. Also the itaconic acid productivity increased significantly. The maximum itaconic acid productivity that was reached under these conditions was $0.15 \mathrm{~g} / \mathrm{L} / \mathrm{h}$, which is only a factor 17 lower than the $2.5 \mathrm{~g} / \mathrm{L} / \mathrm{h}$ that according to the US Department of Energy should be achieved to have an economically feasible production process.

\section{Keywords}

Itaconic acid, Aspergillus niger, modified 6-phosphofructo-1-kinase, cis-aconitate decarboxylase cadA, mitochondrial transporter $m t t A$, plasmamembrane transporter $m f s A$. 


\section{Introduction}

The bio-based production of chemicals from second-generation plant biomass is both economically and scientifically challenging. The cost-efficient production of chemicals requires highly efficient production processes based on highly optimized production organisms. One of the promising production organisms in this respect is Aspergillus niger. This filamentous fungus is industrially used for the production of plant cell wall hydrolyzing enzymes and for the production of metabolites like citrate. Based on its current use, $A$. niger can relatively easily be modified for the production of bio-based chemicals. Itaconic acid is a metabolic derivative of citric acid and therefore can potentially be produced in $A$. niger. Itaconic acid (methyl succinic acid) is a C5 dicarboxylic acid. It is one of the top twelve building block chemicals that can be produced from plant biomass sugars via a fermentative process. ${ }^{1}$ The methylene group of itaconic acid can participate in polymerization reactions and on the basis of this characteristic itaconic acid can be used for the production of synthetic polymers. ${ }^{2}$ It is further used as a bioactive compound in agriculture, pharmacy and as a medicine. ${ }^{3}$ Itaconic acid can also be used as a starting compound for enzymatic transformations to form useful poly-functional building blocks. ${ }^{4}$

Nowadays, Aspergillus terreus is mostly used for the commercial production of itaconic acid in a submerged fermentation process. ${ }^{3,5}$ The metabolic pathway for the production of itaconic acid is similar to the metabolic pathway of citric acid production in A. niger. Given this similarity in biosynthesis in $A$. niger we have started to develop an itaconic acid production process based on $A$. niger citric acid producing strains.

Citric acid is commercially produced using $A$. niger reaching production levels of more than $200 \mathrm{~g} / \mathrm{L}$, while $A$. terreus reaches itaconic acid production levels of $80 \mathrm{~g} / \mathrm{L}$. ${ }^{6}$ Therefore $A$. niger is the host of choice for our research. A. niger is not able to produce itaconic acid naturally since it lacks the essential gene cadA encoding cis-aconitate decarboxylase. The cadA gene was identified via a clone-based transcriptomics ${ }^{7}$ approach and via an enzyme purification ${ }^{8,9}$ approach. In the genome of $A$. terreus two putative transporters flank the cadA gene.

Recently it was shown that especially the putative mitochondrial transporter $m t t A$ is crucial for an efficient itaconic acid production process in $A$. niger and that, to a minor extent, the putative plasmamembrane transporter $m f s A$ also has a positive effect on itaconic acid production. ${ }^{9,10}$ In the past, several research groups tried to increase the flux through the glycolytic pathway since that might greatly enhance the productivity of acid production. The work of Schreferl-Kunar in 1989 already showed that A. niger mutants that were selected on the basis of increased citric acid secretion levels have strongly increased hexokinase and 6-phoshofructo-1-kinase activity. ${ }^{11}$ However, overexpression of 6-phosphofructo-1kinase in a citric acid producing $A$. niger strain did not lead to increased citric acid levels. ${ }^{12}$ Nonetheless, cultivation of $A$. niger on high sugar concentrations showed that the control 
over the glycolytic flux at the level of 6-phosphofructo-1-kinase was absent under these conditions. ${ }^{13}$ Apparently mutations in the 6-phosphofructo-1-kinase gene led to an enzyme that was less inhibited by citrate. This was confirmed by Mlakar and Legiša (2006) who showed that of the two described A. niger 6-phosphofructo-1-kinases ${ }^{14}$ one form was moderately inhibited by citrate while the other form proved to be completely resistant to citrate inhibition. ${ }^{15}$ Later on, a specific truncated version of the $p f k A$ gene from $A$. niger, resistant to citrate inhibition but still highly active, was obtained. ${ }^{16}$ The expression of this modified 6-phosphofructo-1-kinase gene in A. terreus resulted in increased itaconic acid production levels. ${ }^{17}$

In this manuscript, we report the effect of overexpressing the modified 6-phosphofructo1-kinase in $A$. niger on organic acid production, when combined with the cadA gene from $A$. terreus as well as in combination with the itaconic acid biosynthetic cluster cadA, mttA and $m f s A$.

\section{Materials and methods}

\section{Strain}

The fungal strain used in this study was A. niger CAD4 (cspA, $\triangle \arg B, \operatorname{goxC1}$, prtF28) which is deposited at DSMZ with accession number DSM 27587.

\section{Genetic constructs}

The modified 6-phosphofructo-1-kinase gene (accession number HG423570) was obtained by PCR using primer LS_modPFKI_for and LS_modPFKI_rev and A. niger N593 genomic DNA as a template. The PCR fragment was cloned into a pUC19 derived expression vector under control of a modified $x \ln D$ promoter and the $x \ln D$ terminator of A.niger. Plasmids PMTT and pMFS contained the synthetic codon-optimized coding sequences (DNA 2.0) of the putative mitochondrial transporter and putative plasmamembrane transporter respectively both under control of a modified $x \ln D$ promoter $\left({ }^{*}\right)$ and the $x \ln D$ terminator of $A$. niger (accession numbers HG423568 and HG423569 respectively). For the construction of plasmid pLS001 the [p_x $\left.\ln D^{*}-M T T-t \_x \mid n D\right]$ fragment was obtained by PCR using PMTT as a template and primers LS_P_xInD_HindIII_for and LS_t_xInD_Xbal_rev. This fragment was cloned into pMFS, previously digested with HindIII and Xbal. Plasmids were propagated in $\mathrm{DH} 5 \alpha$ E. coli. LB medium supplemented with $100 \mathrm{mg} / \mathrm{L}$ ampicillin was used for E. coli growth.

\section{Fungal transformation}

Protoplasts were generated using Novozyme-234 for the transformation of A. niger CAD4 strain. The pPFKmod plasmid was introduced in $A$. niger by co-transformation as described before ${ }^{18}$, using the pAL69 plasmid containing the $\arg B$ gene as a primary selection marker. 
The pPFKmod plasmid was also introduced together with the pLS001 plasmid by cotransformation using pAL69. Selective MMS plates $\left(6.0 \mathrm{~g} / \mathrm{L} \mathrm{NaNO}_{3}, 1.5 \mathrm{~g} / \mathrm{L} \mathrm{KH}_{2} \mathrm{PO}_{4}, 0.5 \mathrm{~g} / \mathrm{L}\right.$ $\mathrm{KCl}, 0.5 \mathrm{~g} / \mathrm{L} \mathrm{MgSO}_{4} \cdot 7 \mathrm{H}_{2} \mathrm{O}, 1 \mathrm{~mL} / \mathrm{L}$ Vishniac solution, $325.2 \mathrm{~g} / \mathrm{L}$ sucrose, $1.2 \%$ (w/v) agar, $\mathrm{pH}$ 6.0) were used to select for protoplasts that took up the selection marker plasmid and possibly the pPFKmod plasmid and/or the pLS001 plasmid. Colonies were randomly picked from the transformation plates and re-plated on complete medium. ${ }^{19}$

\section{Selection of transformants}

Fresh mycelium was disrupted using Fastprep with glass beads and $400 \mu \mathrm{L}$ DNA extraction buffer (0.1 M Tris $\mathrm{HCl}$ pH 8.0, 1.2 M NaCl, 5 mM EDTA).

DNA was extracted using phenol-chloroform extraction. The pellet was washed with $70 \%$ cold ethanol, air-dried and re-suspended in $50 \mu \mathrm{L} \mathrm{MQ}$ water.

Transformants were selected by PCR using the extracted genomic DNA as a template. The primers LS_pPFK1_for, located in the pfkA gene and LS_txInD_rev located in the $x \ln D$ terminator, were used to check for the presence of pPFKmod. The presence of the putative mitochondrial transporter mttA was confirmed using primers LS_pMTT_for and LS_pMTT_rev whereas the presence of the putative plasmamembrane transporter $m f s A$ was confirmed with primers LS_pMFS_for and LS_pMFS_rev (Table 4.1).

The transformants containing the genes of interest were re-plated on complete medium. Fungal spores were harvested after 4 days of growth at $30^{\circ} \mathrm{C}$.

Table 4.1 Sequences of primers used in this study.

\begin{tabular}{ll}
\hline Primername & Sequence \\
\hline LS_modPFKI_for & GAGAATGCATATGGCTCCCCCCCAAGC \\
LS_modPFK_rev & GAGAGCGGCCGCATCATAGTGCCGGCACAGACC \\
LS_p_xInD_HindIII_for & GAGAAAGCTTCGAATGAGGAGGTGTTGCAG \\
LS_t_xInD_Xbal_rev & GAGATCTAGACTGCAGTCGCACTCCCGACC \\
LS_pPFK1_for & TGACATGTGCGCTATCATTACC \\
LS_txInD_rev & TCCCCTAGAGCCATCAACAG \\
LS_pMTT_for & ATTAAGACCCGCATGCAATC \\
LS_pMTT_rev & CTTCTCGTAGACGGGGAACA \\
LS_pMFS_for & ACCTTCACTAGCTGGCGTGT \\
LS_pMFS_rev & GACATCCGTGGGACTGAACT \\
LS_q_pfkA_F & CGTGAGAACAAGATCTTGCG \\
LS_q_pfkA_R & CGCATTCTCTTGTTCTCTGG \\
LS_q_gotra_F & TTTTCAGTCTGGCTGCTCCT \\
LS_q_gotra_R & CTGTTTTCCTGCATCGTGTG \\
LS_q_kan_F & AGCATTACGCTGACTTGACG \\
LS_q_kan_R & AGGTGGACCAGTTGGTGATT \\
\hline
\end{tabular}




\section{Growth experiment}

The different $A$. niger transformants and controls were grown, after inoculation of $10^{6}$ spores per $\mathrm{mL}$, in $1 \mathrm{~L}$ Erlenmeyer flasks containing $200 \mathrm{~mL}$ PM medium (1.2 g NaNO${ }_{3}, 0.5$ $\mathrm{g} \mathrm{KH}_{2} \mathrm{PO}_{4}, 0.2 \mathrm{~g} \mathrm{MgSO}_{4} 7 \mathrm{H}_{2} \mathrm{O}, 0.5 \mathrm{~g}$ Yeast extract and $40 \mathrm{uL}$ Vishniac ${ }^{20}$ per liter) ${ }^{21}$ with 100 $\mathrm{mM}$ sorbitol as a carbon source. The expression of the different recombinant genes studied in this work was induced, 18 hours after inoculation ( $t=0)$, with $50 \mathrm{mM}$ xylose. All A. niger strains were grown for 5 days at $30^{\circ} \mathrm{C}$ and $250 \mathrm{rpm}$. Samples for HPLC analysis were taken at $6 \mathrm{~h}, 30 \mathrm{~h}, 54 \mathrm{~h}$ and $78 \mathrm{~h}$ after induction.

\section{Metabolite analysis with HPLC}

High-performance liquid chromatography (HPLC) was used to determine the extracellular concentrations of sorbitol, xylose, citric acid, itaconic acid, cis-aconitic acid, pyruvic acid, $\alpha$-ketoglutaric acid, lactic acid, succinic acid, fumaric acid and oxalic acid. A Thermo Accela equipped with a Shodex KC-811 column was used. Separations were performed by isocratic elution with $0.01 \mathrm{~N} \mathrm{H}_{2} \mathrm{SO}_{4}$ at a flow rate of $0.8 \mathrm{~mL} \mathrm{~min}{ }^{-1}$. The detection of the different compounds was done using both a refractive index detector (Spectrasystem RI-150, sample frequency $5.00032 \mathrm{~Hz}$ ) and a UV-VIS detector (Spectrasytem UV1000, $\lambda=210 \mathrm{~nm}$ ). Crotonate (6 $\mathrm{mM})$ was used as an internal standard.

\section{RNA extraction and CDNA preparation}

Frozen mycelium was placed in $2 \mathrm{~mL}$ tubes prefilled with $1 \mathrm{~mm}$ silica spheres (Lysing Matrix $C, M P$ ) and homogenized in $1 \mathrm{~mL}$ of peqGOLD TriFast DNA/RNA/protein purification system reagent. A FastPrep-24 Instrument (MP) was used to distupt the mycelium. RNA was isolated according to the manufacturer's instructions. The extracted total RNA was spiked with a synthetic control RNA transcript, a bacterial kanamycin synthetase-encoding gene fused to a eukaryotic poly (A) tail (Promega), to correct for various efficiencies of reverse transcription or PCR itself. ${ }^{23}$ CDNA was synthesized using the RevertAid $\mathrm{H}$ Minus first-strand cDNA synthesis kit (Thermo Scientific) according to the manufacturer's instructions.

\section{qPCR analysis}

Transcript levels were determined in triplicate for the gene of interest ( $p f k A$ ) as well as for a reference gene (GOTRA) and the synthetic control RNA transcript using the Rotor-Gene Q Cycler. The qPCR mixture contained $7.5 \mu \mathrm{L} 2 x$ Absolute QPCR SYBR Green mix (Thermo Scientific), $100 \mathrm{nM}$ forward and reverse primers and $2.5 \mu \mathrm{L} 100$ times diluted cDNA. Primers LS_q_pfkA_F and LS_q_pfkA_R were used to determine the transcript level of the $p f k A$ gene and primers LS_q_gotra_F and LS_q_gotra_F were used to determine the transcript level of the reference gene An02g04120. ${ }^{22}$ The transcript levels of the synthetic kanamycin gene were determined using primers LS_q_kan_F and LS_q_kan_R (Table 4.1).

Water and SDS samples were used as controls. The qPCR cycling program was as follows; $15 \mathrm{~min}$ initial polymerase activation at $95^{\circ} \mathrm{C}$ followed by 40 cycles of $95^{\circ} \mathrm{C}$ for $15 \mathrm{~s}, 58^{\circ} \mathrm{C}$ for 
$15 \mathrm{~s}$ and $72^{\circ} \mathrm{C}$ for $20 \mathrm{~s}$. The calculations were done using the method of Pfaffl ${ }^{24}$ using the expression level of pfkA in the parent strain CAD as the reference.

\section{Results}

\section{Transformants harboring cadA and the modified $p f k A$ gene}

Extracellular itaconic acid and citric acid levels were monitored during a time course experiment that included seven $A$. niger CAD4 transformants (named T1 to T7), carrying both the cadA gene from $A$. terreus and the modified $p f k A$ gene from $A$. niger, plus the parent strain as a control. Overexpression of the modified $p f k A$ gene in the $A$. niger cadA harboring strain led to a strongly increased citric acid production when compared to the parent strain CAD (Fig. 4.1).

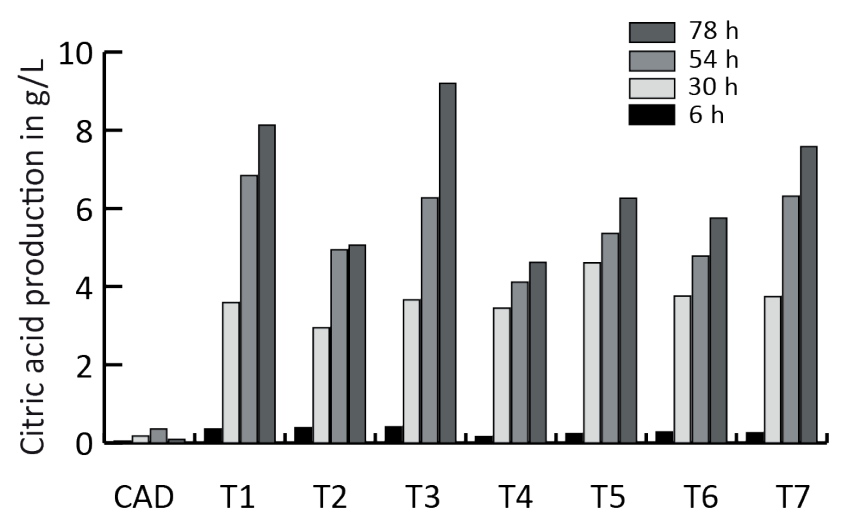

Figure 4.1 Citric acid production in $\mathrm{g} / \mathrm{L}$ of the transformants harboring cadA and the modified $p f k A$ followed in time $(6,30,54,78$ hours after induction).

All transformants produced significantly higher levels of citric acid. The levels of citric acid produced range between 4.6 and $9.2 \mathrm{~g} / \mathrm{L}$ while the parent strain produced only $0.1 \mathrm{~g} / \mathrm{L}$ under these conditions. However, in contrast to the high increase in observed citric acid production, the combination of the modified pfkA gene and the cadA gene from $A$. terreus did not lead to an increase in itaconic acid levels, as shown in Fig. 4.2. 


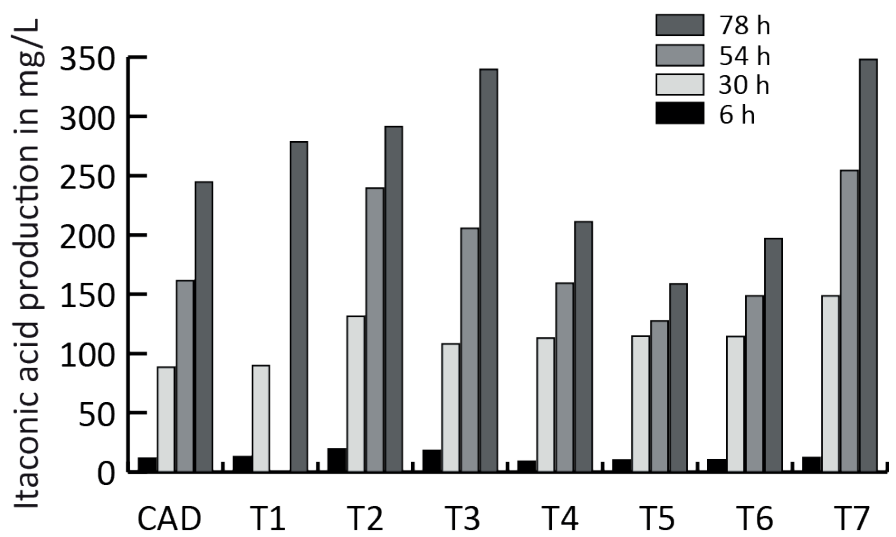

Figure 4.2 Itaconic acid production in $\mathrm{mg} / \mathrm{L}$ of the transformants harboring cadA and the modified $p f k A$ followed in time $(6,30,54$, 78 hours after induction).

Four of the analyzed transformants produce slightly more itaconic acid as compared to the parental strain, while three of the transformants produced less itaconic acid 78 hours after induction.

The parental strain reached extracellular itaconic acid levels of $240 \mathrm{mg} / \mathrm{L}$ while the transformants produced between 159 and $348 \mathrm{mg} / \mathrm{L}$. The combined C-mol yield of citric acid and itaconic acid is strongly improved as a result of the introduction of the modified pfkA gene (Fig. 4.3). The best performing transformant reached a yield of $35 \%$.

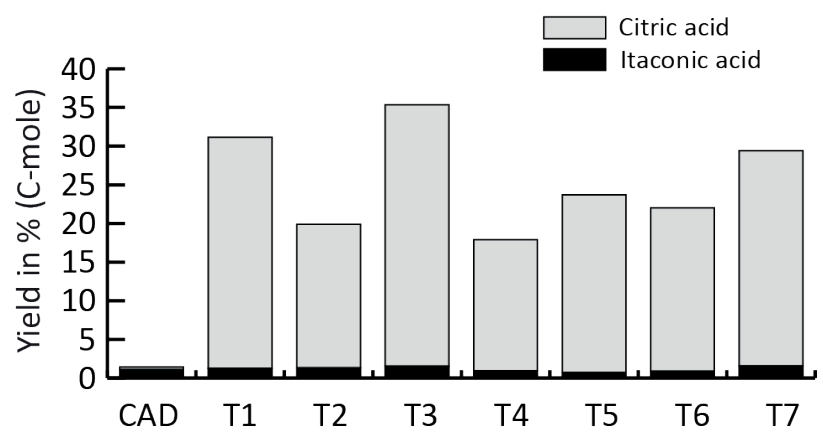

Figure 4.3 Calculated C-mole yield (\%) of itaconic acid and citric acid for each transformant harboring the modified pfkA gene in combination with cadA compared to the control strains that only contain the cadA gene at $\mathrm{t}=78 \mathrm{~h}$. 
Transformants expressing the itaconic acid biosynthetic cluster cadA, $m t t A, m f s A$ in combination with the modified $p f k A$ gene

Both the strains harboring the itaconic acid production cluster cadA, $m t t A$ and $m f s A$ (C series) as well as the transformants carrying the modified $p f k A$ gene together with the itaconic acid biosynthetic cluster ( $T$ series) produce very variable amounts of citric acid (Fig. 4.4). The largest difference was found in the strains that express the itaconic acid biosynthetic cluster, in which the citric acid level varies between 0.2 and $5.5 \mathrm{~g} / \mathrm{L}$ at 78 hours after induction.

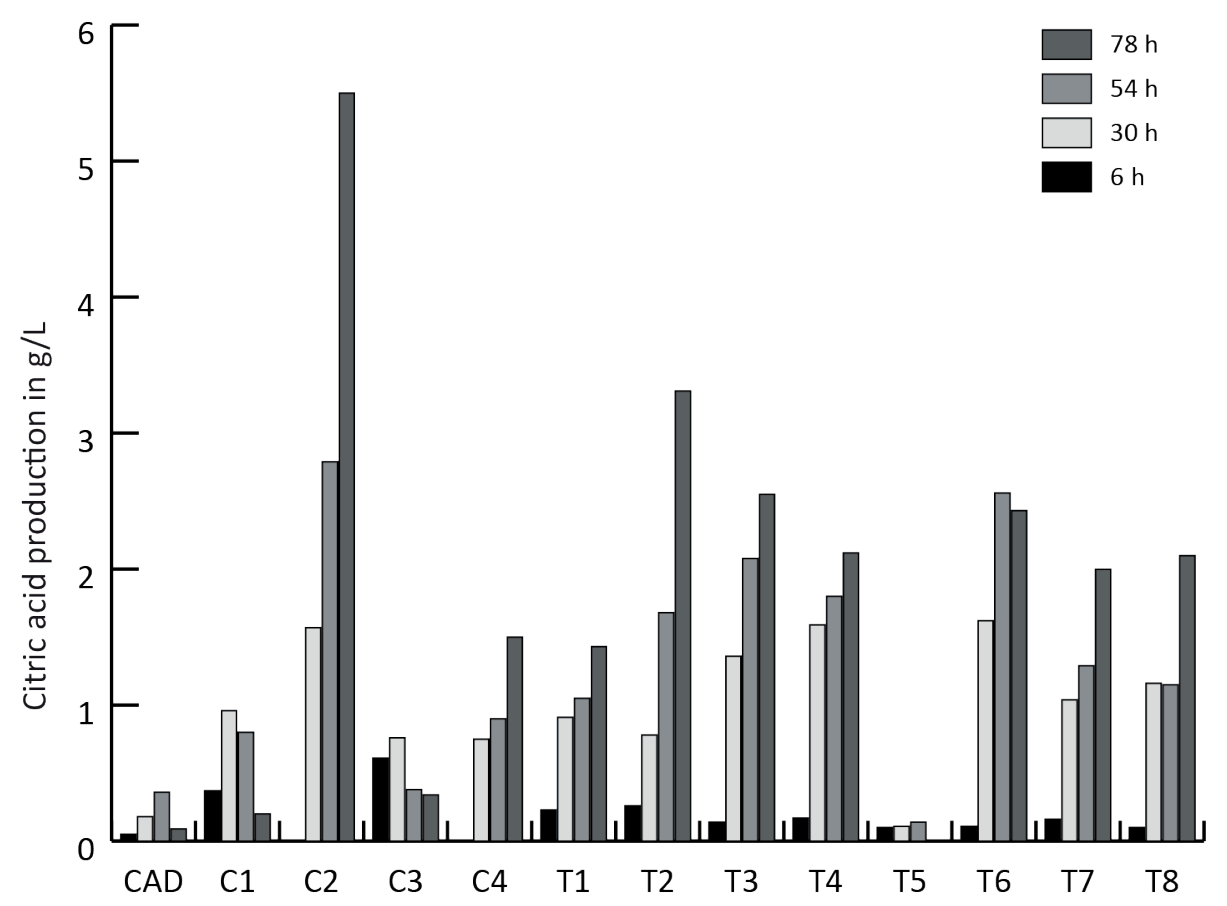

Figure 4.4 Citric acid production in $\mathrm{g} / \mathrm{L}$ of transformants containing the itaconic acid biosynthesis cluster cadA, $m t t A$ and $m f S A$ (C1C4) compared to transformants harboring in addition the modified $p f k A$ gene (T1-T8). Aliquots for citric acid analysis were taken in time $(6,30,54,78$ hours after induction).

The strains that express the modified pfkA in combination with the itaconic acid biosynthetic cluster produce significantly higher amounts of itaconic acid (Fig. 4.5) at sampling time point $30 \mathrm{~h}$ and $54 \mathrm{~h}$ after induction compared to the strains that only harbor the itaconic acid biosynthetic cluster (T-test, $p=0.04$ for $30 \mathrm{~h}$ and $p=0.003$ for $54 \mathrm{~h}$ ). At $t=78 \mathrm{~h}$ after induction there were no significantly increased levels of itaconic acid observed $(p=0.15)$. 


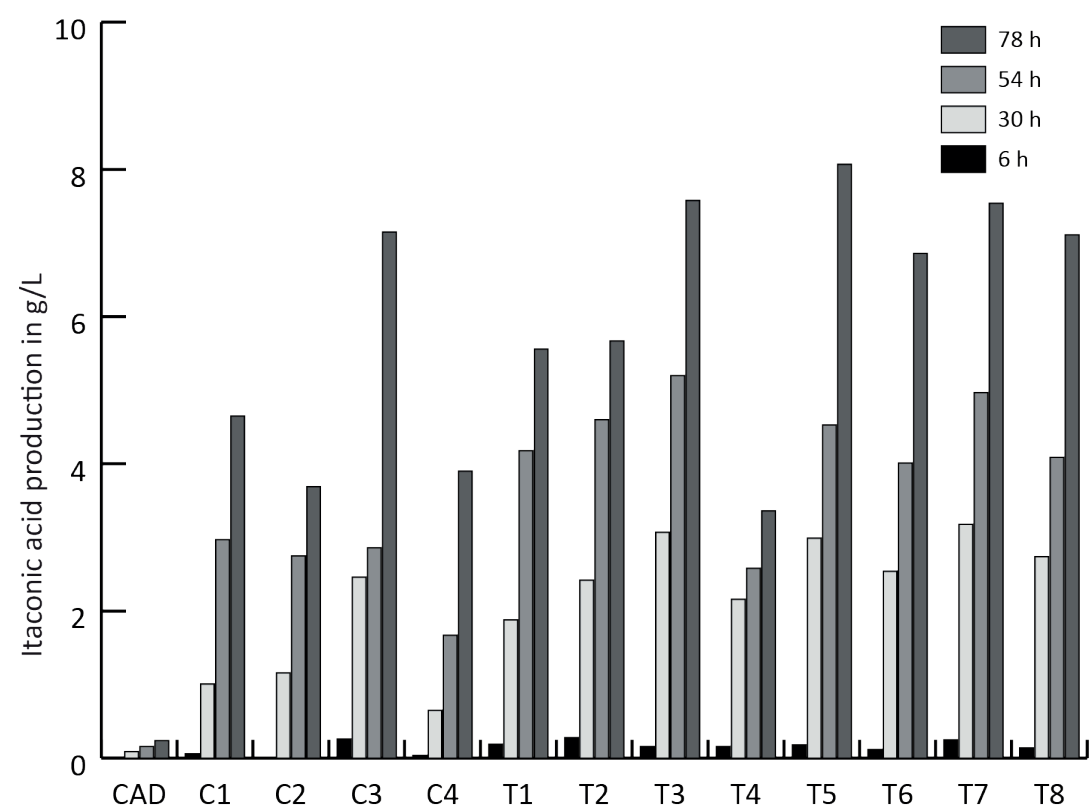

Figure 4.5 Itaconic acid production in $\mathrm{g} / \mathrm{L}$ of transformants containing the itaconic acid biosynthesis cluster cadA, $m t t A$ and $m f s A$ (C1-C4) compared to transformants harboring in addition the modified pfkA gene (T1-T8). Aliquots for itaconic acid analysis were taken in time $(6,30,54,78$ hours after induction).

The total yield, expressed in C-mol, of itaconic acid and citric acid in the cluster strains (C series) ranged between $22 \%$ and $37 \%$ while the yield of the cluster plus the modified $p f k A$ transformants (T series) ranged between $23 \%$ and $44 \%$ at $t=78 \mathrm{~h}$. This was statistically not significantly higher $(p=0.14)$ (Fig. 4.6).

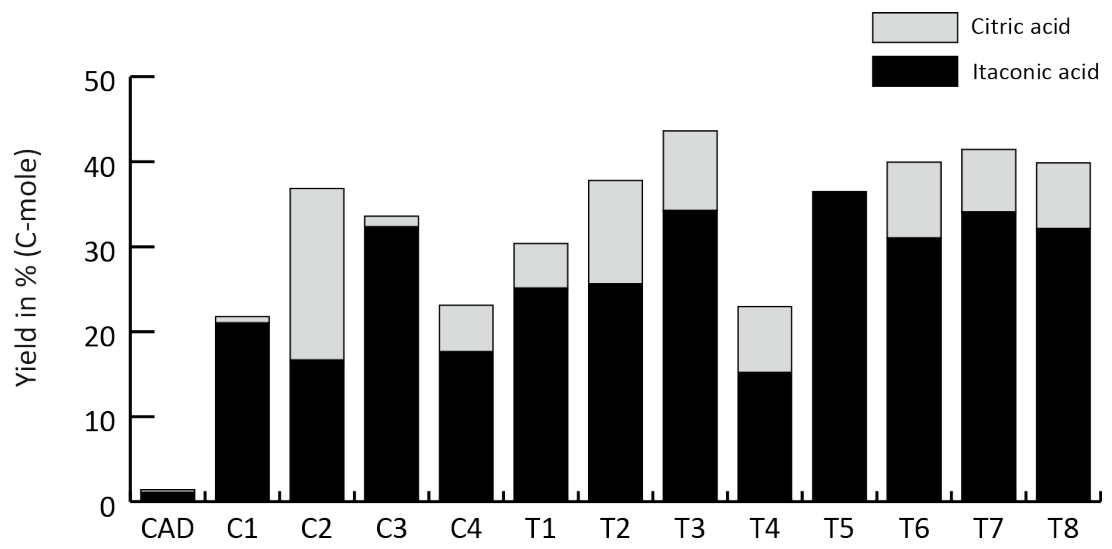

Figure 4.6 Calculated C-mol yield (\%) of itaconic acid and citric acid for each transformant harboring the complete itaconic acid biosynthesis cluster $c a d A, m t t A$ and $m f s A$ respectively and the modified $p f k A(T 1-T 8)$ compared to the control strains that only contain the $\operatorname{cad} A, m t t A$ and $m f s A$ genes $(\mathrm{C} 1-\mathrm{C} 4)$ at $\mathrm{t}=78 \mathrm{~h}$. 
The total C-mol yield of itaconic acid and citric acid was only significantly higher for the $p f k A$ transformants at $\mathrm{t}=30 \mathrm{~h}$ and $\mathrm{t}=54 \mathrm{~h}(\mathrm{p}=0.03$ and $\mathrm{p}=0.03$ respectively, data not shown). In addition, the productivity levels were significantly higher $(p=0.03)$ for the transformants expressing the itaconic acid biosynthetic cluster and the modified $p f k A$ gene compared to the strains that lack the modified pfkA gene.

The highest itaconic acid productivity level within the modified $p f k A$ recombinant strains ( $T$ series) was reached 78 hours after induction by one single transformant (147 mg/L/h), while, on average, the highest productivity levels for this group of strains was already reached after 30 hours (102 mg/L/h) (Fig. 4.7).

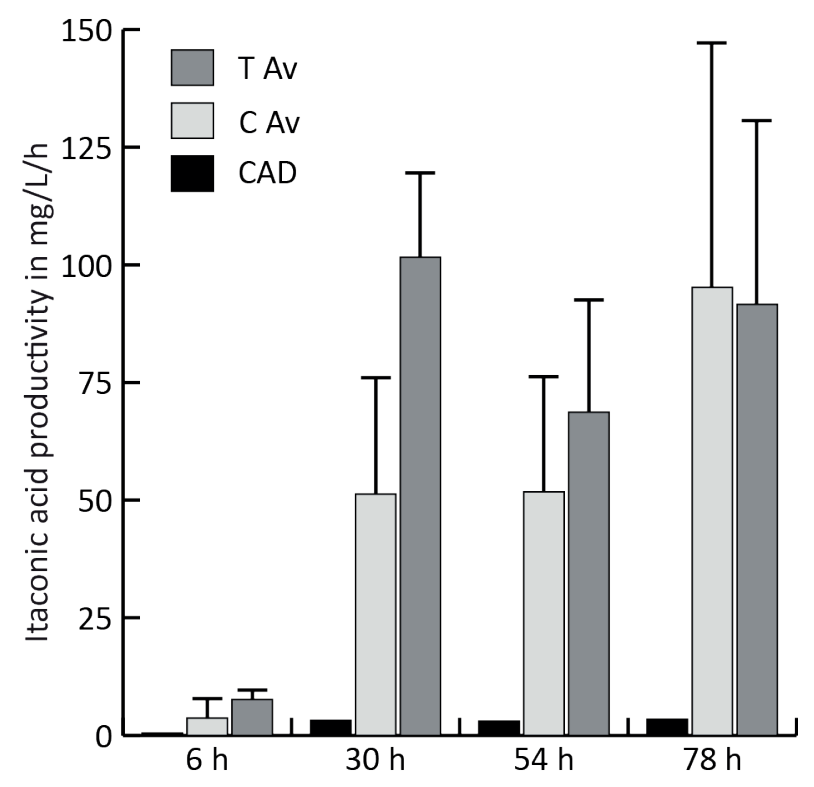

Figure 4.7 Average itaconic acid productivity levels in $\mathrm{mg} / \mathrm{L} / \mathrm{h}$ for the transformants harboring the cluster genes cadA, $m t t A, m f s A$ and the modified $p f k A(T A v)$, compared to the average productivity of the transformants harboring only the cluster genes cad $A$, $m t t A, m f s A(C A v)$. At time point $t=30 \mathrm{~h}$ the difference in productivity is significant ( $T$-Test, $\mathrm{p}=0.03$ ).

The total organic acid productivity, expressed in C-mmol/L/h (Fig. 4.8), increased significantly at $\mathrm{t}=30 \mathrm{~h}(\mathrm{p}=0.03)$ for the transformants (T series) compared to the strains harboring only the cluster genes (C series). The maximum total organic acid productivity at $\mathrm{t}=30 \mathrm{~h}$ was 6.25 $\mathrm{C}-\mathrm{mmol} / \mathrm{L} / \mathrm{h}$ and corresponded to one of the transformants harboring the cluster genes and the modified $p f k A$ gene, while the best producing strain, expressing only the itaconic acid biosynthetic cluster, achieved a maximum of $3.9 \mathrm{C}-\mathrm{mmol} / \mathrm{L} / \mathrm{h}$. 


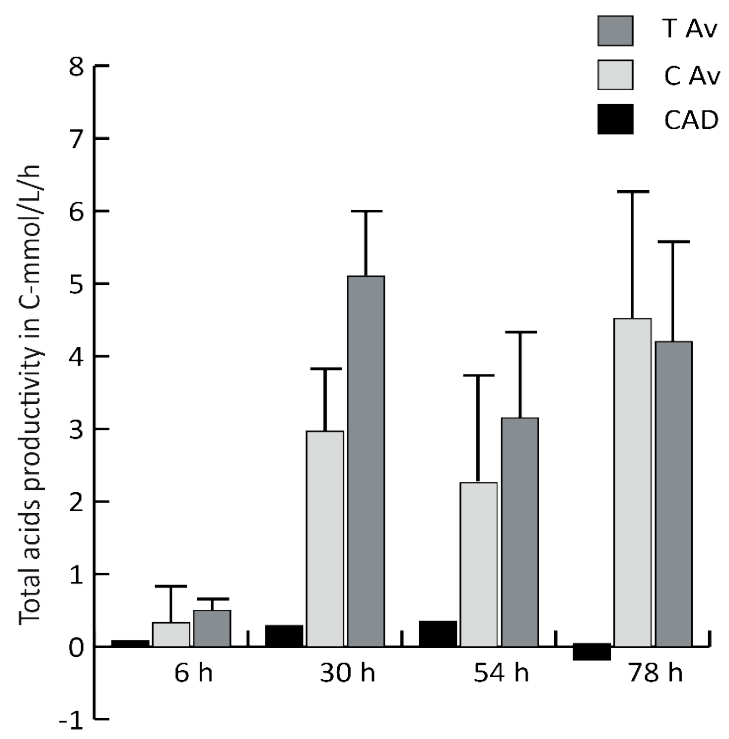

Figure 4.8 The average total acid production in C-mmol/L/h for the transformants harboring the cluster genes cadA, $m t t A, m f s A$ and the modified $p f k A$ ( $T A v$ ) compared to the average productivity of the transformants harboring only the cluster genes cadA, $m t t A$, $m f s A(C A v)$. At time point $\mathrm{t}=30 \mathrm{~h}$ the difference in productivity is significant ( $\mathrm{T}$-Test, $\mathrm{p}=0.01$ ).

The transcription ratios of the $p f k A$ gene of the three transformants that gave the highest itaconic acid productivity at time point $30 \mathrm{~h}$ (T3, T5 and T7) were compared with the highest itaconic acid producing strain that expresses the cluster genes $c a d A, m t t A$ and $m f s A(C 3)$ and with the parent strain CAD. The results showed that there is no difference in $p f k A$ transcription between the CAD strain and the $\mathrm{C} 3$ strain. The transformants T3, T5 and T7 gave an increased transcription level of a factor 5.2, 5.6 and 4.3 respectively, compared to the parent strain CAD (Fig. 4.9).

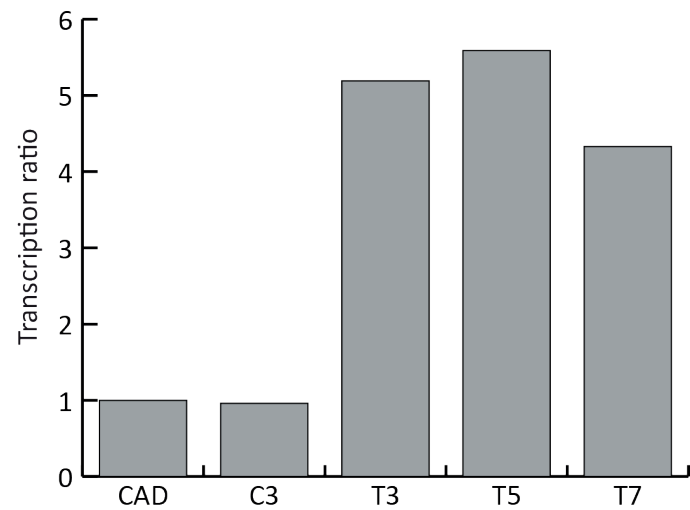

Figure 4.9 Relative transcription levels of the $p f k A$ gene in the different transformants compared to the parent strain CAD that was used as the reference strain. Samples were taken 30 hours after induction, which was the time point where the highest itaconic acid productivity was observed. $C 3$ is the strain that contains the cluster genes cadA, $m t t A$ and $m f S A$. The transformants T3, T5 and $T 7$ are the strains that express the cluster genes cadA, $m t t A$ and $m f s A$ as well as the modified $p f k A$. 


\section{Discussion}

Our results show transformants that overexpress the modified $p f k A$ gene in combination with cadA have significantly increased citrate secretion levels. This confirms that the modified $p f k A$ gene has a similar effect on citric acid production in our strain compared to what was found before by Capuder et al. ${ }^{16}$ They were able to show that the overexpression of the modified pfkA gene in the $A$. niger 158 strain resulted in a $70 \%$ increase in citrate secreted into the fermentation broth after 300 hours of growth.

In our research the effect was far more evident but since the experimental set-up was quite different it is difficult to compare both results. The overexpression of the modified $p f k A$ in the CAD strain also had a strong effect on the citrate yield: levels up to $35 \%$ were achieved while the CAD strain only reached a yield of about $2 \%$. As shown before, in particular the putative mitochondrial transporter $m t t A$ and to a lesser extent also the putative plasmamembrane transporter $m f s A$ play a crucial role in itaconic acid production. ${ }^{9,10}$ This was confirmed by the results reported in this work, since itaconic acid production in the strains harboring the modified $p f k A$ gene and the cadA gene from $A$. terreus was not improved when compared to the parent strain that only harbored the cadA gene. Since both transporters are crucial for itaconic acid production, the modified $p f k A$ gene was also co-expressed with the complete itaconic acid biosynthetic cluster of $A$. terreus consisting of cadA, $m t t A$ and $m f s A$. The effect of these metabolic modifications on citrate production varied. This can be expected because the construct carrying the genes is randomly integrated in the genome of $A$. niger and its copy number varies.

Nevertheless, we do find an effect of these metabolic modifications on itaconic acid production. The transformants series harboring the modified $p f k A, \operatorname{cad} A, m t t A$ and $m f s A$ produced significantly more itaconic acid than the strains harboring cadA, $m t t A$ and $m f s A$ both at 30 and 54 hours after induction. However, 78 hours after induction the itaconic acid production of the $T$ series and the $C$ series strains did not significantly differ anymore. This indicates that the modified 6-phosphofructo-1-kinase has a positive effect on itaconic acid production only in the early stages of production. The average highest itaconic acid productivity reached by the $T$ series transformants also occurred at early time points. In particular, at 30 hours after induction, a significant difference in productivity, caused by the overexpression of the modified $p f k A$ gene, was observed.

The transcription levels were determined for the $p f k A$ gene using a set of primers that bind both to the cDNA of the endogenous $p f k A$ gene as well as to the cDNA of the modified $p f k A$ gene. This assumes that the transcription levels of the endogenous $p f k A$ gene do not change after transformation. The transcription ratio (1.0) of the strain harboring the cluster genes cadA, mttA and $m f s A$ compared to the parent strain cadA (1.0) showed that the expression of the transporters do not influence the transcription level of $p f k A$.

A positive effect on the itaconic acid productivity was also observed when the modified $p f k A$ gene from A. niger was expressed in Aspergillus terreus. ${ }^{17}$ As suggested in the report of the 
US Department of Energy ${ }^{1}$ an itaconic acid productivity of $2.5 \mathrm{~g} / \mathrm{L} / \mathrm{h}$ should be achieved before the process is economically feasible. Under the conditions tested in the present study the productivity was only a factor 17 lower than the desired productivity.

Since our growth conditions are merely designed to test the effects of the metabolic modifications at lab scale, several improvements, such as the use of more optimal culture conditions and $A$. niger genetic backgrounds, could be done in order to increase the itaconic acid production to the desired industrial levels. In this study growth media contained 100 $\mathrm{mM}$ sorbitol and $50 \mathrm{mM}$ xylose as a carbon source and inducer, while glucose-based media are predominantly used for the production of citric acid and itaconic acid. Using different carbon sources in higher concentrations might further improve the itaconic acid productivity. The strain we used is not an industrial citrate-producing strain and the growth experiment in this study was done in shake flasks. In summary, the usage of an industrial citrate-producing $A$. niger strain in an optimized and controlled fermentation process should allow itaconic acid productivity levels of $2.5 \mathrm{~g} / \mathrm{L} / \mathrm{h}$ and thus create an economically feasible production process.

\section{Competing interests}

The authors declare that they have no competing interests.

\section{Acknowledgements}

This work has been carried out on the basis of a grant in the framework of the BE BASIC program F01.002 Itaconic/fumaric acids: Novel economic and eco-efficient processes for the production of itaconic and fumaric acid. 


\section{References}

1. Werpy T, Petersen G. Top value added chemicals from biomass: Volume I-Results of screening for potential candidates from sugars and synthesis gas. 2004:1-76.

2. Kubicek CP, Punt PJ, Visser J. Production of organic acids by filamentous fungi. The Mycota. Industrial Applications, Edited by M. Hofrichter, Berlin, Heidelberg: Springer Berlin Heidelberg. 2010:215-234

3. Okabe M, Dwiarti L, Kanamasa S, Park EY. Biotechnological production of itaconic acid and its biosynthesis in Aspergillus terreus. Appl Microbiol Biotechnol 2009,84:597-606.

4. Ferraboschi P, Casati S, Grisenti P, Santaniello E. Selective enzymatic transformations of itaconic acid derivatives: An access to potentially useful building blocks. Tetrahedron 1994,50:3251-3258.

5. Willke T, Vorlop KD. Biotechnological production of itaconic acid. Appl Microbiol Biotechnol 2001,56:289-295.

6. Kuenz A, Gallenmüller Y, Willke T, Vorlop K-D. Microbial production of itaconic acid: developing a stable platform for high product concentrations. App/ Microbiol Biotechnol 2012,96:1209-1216.

7. Li A, van Luijk N, ter Beek M, Caspers M, Punt P, van der Werf M. A clone-based transcriptomics approach for the identification of genes relevant for itaconic acid production in Aspergillus. Fungal Genet Biol 2011,48:602-611.

8. Kanamasa S, Dwiarti L, Okabe O, Park EY. Cloning and functional characterization of the cisaconitic acid decarboxylase (CAD) gene from Aspergillus terreus. Appl Microbiol Biotechnol 2008,80:223-229.

9. van der Straat L, Vernooij M, Lammers M, van den Berg WAM, Schonewille T, Cordewener J, et al. Expression of the Aspergillus terreus itaconic acid biosynthesis cluster in Aspergillus niger. Microbial Cell Fact 2014,13:1-9.

10. Li A, Pfelzer N, Zuijderwijk R, Brickwedde A, van Zeijl C, Punt P. Reduced by-product formation and modified oxygen availability improve itaconic acid production in Aspergillus niger. Appl Microbiol Biotechnol 2013,97:3901-3911.

11. Schreferl-Kunar G, Grotz M, Röhr M, Kubicek CP. Increased citric acid production by mutants of Aspergillus niger with increased glycolytic capacity. FEMS Microbiol Lett 1989,59:297-300.

12. Ruijter GJG, Panneman H, Visser J. Overexpression of phosphofructokinase and pyruvate kinase in citric acid-producing Aspergillus niger. Biochim Biophys Acta 1997,1334:317-326.

13. Peksel A, Torres NV, Liu J, Junea G, Kubicek CP. ${ }^{13} \mathrm{C}-\mathrm{NMR}$ analysis of glucose metabolism during citric acid production by Aspergillus niger. Appl Microbiol Biotechnol, 2002,58:157-163.

14. MesojednikS, Legiša M. Posttranslational modification of 6-phosphofructo-1-kinase in Aspergillus niger. Appl Environ Microbiol 2005,71:1425-1432.

15. Mlakar T, Legiša M. Citrate inhibition-resistant form of 6-phosphofructo-1-kinase from Aspergillus niger. Appl Environ Microbiol 2006,72:4515-4521.

16. Capuder $M$, Šolar $T$, Benčina $M$, Legiša $M$. Highly active, citrate inhibition resistant form of Aspergillus niger 6-phosphofructo-1-kinase encoded by a modified pfkA gene. J Biotechnol 2009,144:51-57. 
17. Tevž G, Benčina M, Legiša M. Enhancing itaconic acid production by Aspergillus terreus. Appl Microbiol Biotechnol 2010,87:1657-1664.

18. Kusters-van Someren MA, Harmsen JA, Kester HC, Visser J. Structure of the Aspergillus niger pelA gene and its expression in Aspergillus niger and Aspergillus nidulans. Curr Genet 1991,20:293-299.

19. Pontecorvo G, Roper JA, Hemmons LM, MacDonald KD, Bufton AWJ. The genetics of Aspergillus nidulans. Adv Genet 1953,5:141-238.

20. Vishniac W, Santer M. The thiobacilli. Bacteriol Rev 1957,21:195-213.

21. Ruijter GJ, van de Vondervoort PJ, Visser J. Oxalic acid production by Aspergillus niger: an oxalate-non-producing mutant produces citric acid at pH 5 and in the presence of manganese. Microbiology 1999,145:2569-2576.

22. van der Veen D, Oliveira JM, van den Berg WAM, de Graaff LH. Analysis of variance components reveals the contribution of sample processing to transcript variation. Appl Environ Microbiol 2009,75:2414-2422.

23. Huggett J, Dheda K, Bustin S, Zumla A. Real-time RT-PCR normalisation; strategies and considerations. Genes and Immunity 2005,6:279-284.

24. Pfaffl MW. A new mathematical model for relative quantification in real-time RT-PCR. Nucl Acids Res 2001,29:e45. 



\section{6}

(9)
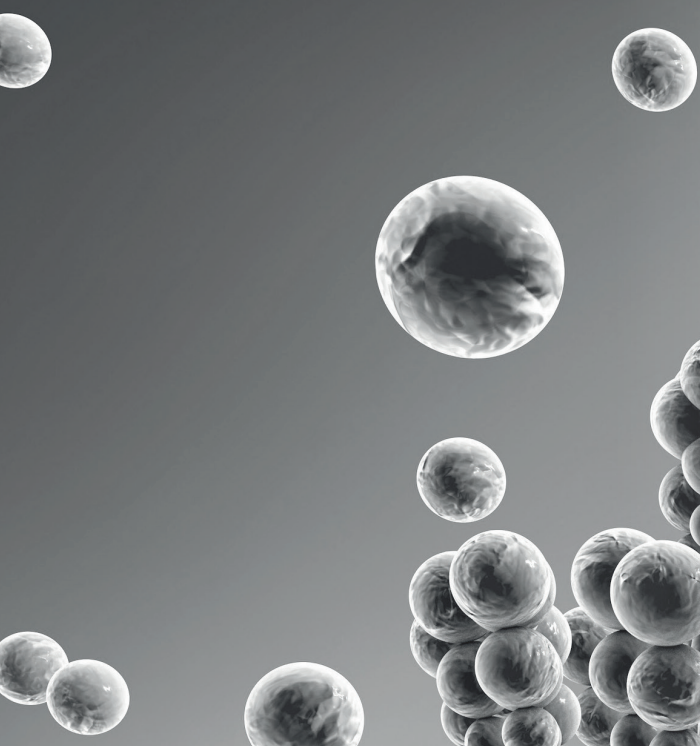

6

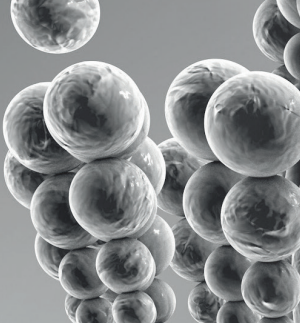

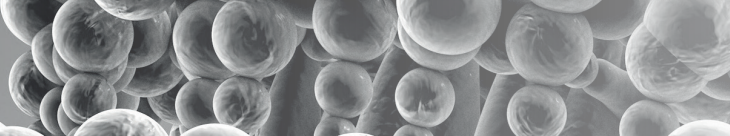
(1)
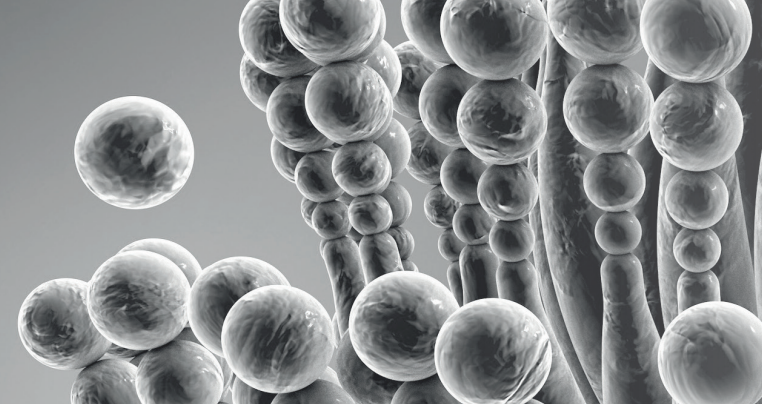

(9)
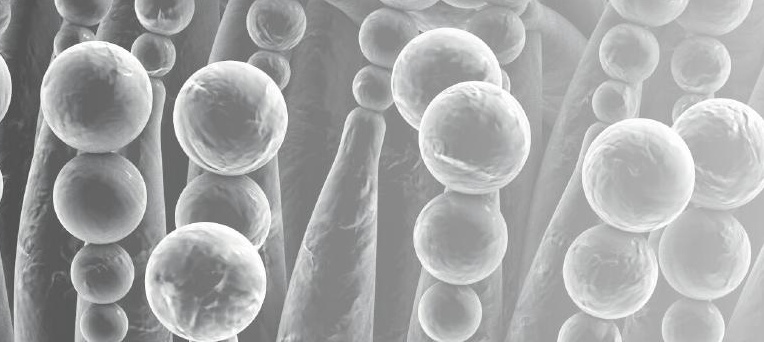

$$
\text { Q }
$$
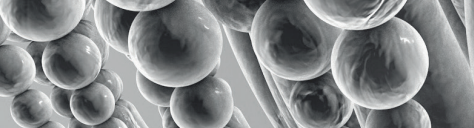

428

init 180

il mo

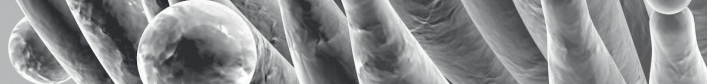

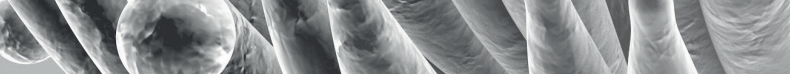

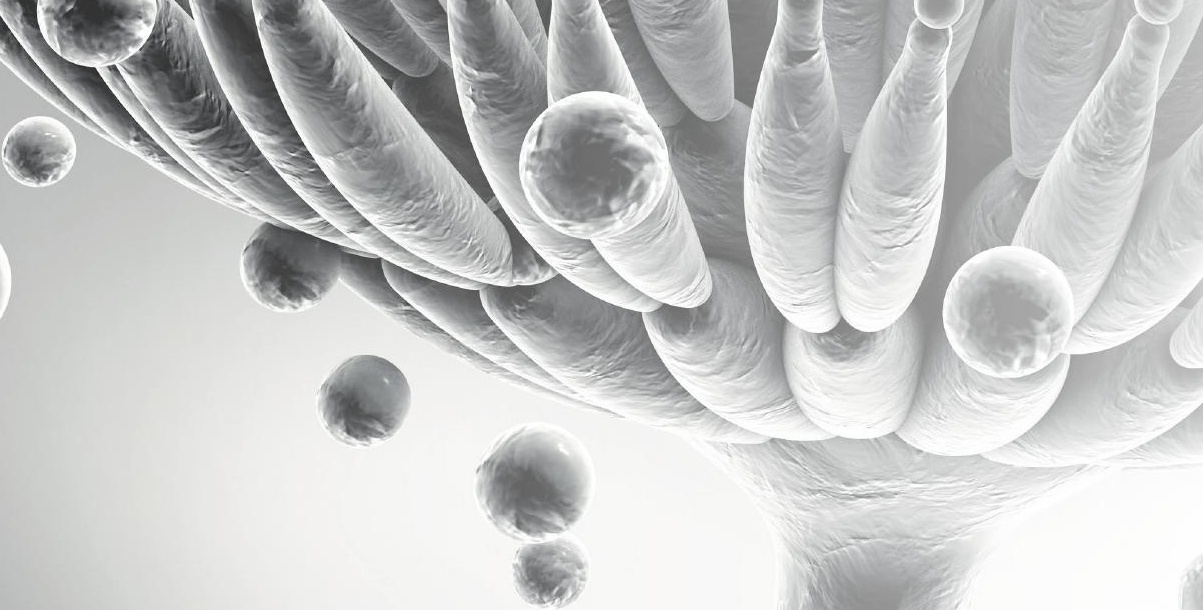

(.

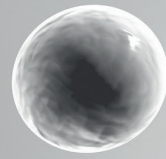

6 


\title{
Chapter 5
}

\author{
Funbricks: design of versatile \\ expression vectors for synthetic \\ biology of Aspergillus niger
}




\section{Abstract}

In the field of synthetic biology there is a need for efficient flexible pathway transfer systems for filamentous fungi. Here we describe the Funbrick system that enables easy and quick construction of expression vectors for integration of pathways and the construction of localization vectors. A major advantage of this system is the ability to freely alter the Funbrick after its construction.

The Funbrick system was successfully applied by integration of the itaconic acid biosynthesis gene cluster of Aspergillus terreus in the genome of Aspergillus niger. Expression of the inserted cadA, mttA and $m f s A$ genes was confirmed by qPCR and itaconic acid production levels were quantified using HPLC. The highest itaconic acid production found was $169 \mathrm{mg} / \mathrm{L}$. Besides itaconic acid, also citric acid and cis-aconitic acid were found in the supernatant varying in concentrations between 0.2 and $1.6 \mathrm{~g} / \mathrm{L}$ citric acid and 0.8 and $2.2 \mathrm{~g} / \mathrm{L}$ cis-aconitic acid.

Localization of the itaconic acid biosynthesis proteins was shown using the localization Funbricks. CadA was localized in the cytosol, de mitochondrial transporter MttA was indeed localized in the mitochondria and the putative plasmamembrane transporter MfsA surprisingly was found being localized in membrane-like structures, but not in the plasmamembrane, cytosol or mitochondria.

In this research we showed the design of the Funbricks. Although the itaconic acid production level was relatively low, the Funbrick expression vectors were successfully used to transfer the itaconic acid biosynthesis cluster in one transformation event to A. niger and to localize CadA, MttA and MfsA.

\section{Keywords}

Funbricks, itaconic acid, Aspergillus niger, GFP localization, pathway transfer 


\section{Introduction}

\section{Biobricks and standardization}

Within the field of synthetic biology there is a growing interest in the use of filamentous fungi for the production of pharmaceuticals and commodity chemicals. Despite the beneficial characteristics of filamentous fungi as production hosts or chassis in biotechnological processes, development is hindered by a relatively limited availability of molecular biology tools. ${ }^{1}$ The rapid development of omics technologies opens new avenues for the study of metabolic networks in filamentous fungi. To optimally benefit from these developments, efficient tools are needed that allow fast, efficient and predictable network redesign. One such tool involves the development of a standardized vector or Biobrick system, as e.g. developed for the model organisms Escherichia coli and Saccharomyces cerevisiae (www.partsregistry.org). In general, a Biobrick system consists of standardized parts like promoters, coding sequences, terminators and transformation markers. Each part can be optimized, like a coding sequence for chassis-specific codon-usage or a promoter that is relieved of a negative feedback mechanism. From these basic expression devices many variants can be derived, like pathway devices that express a metabolic pathway, reporter devices that show the cellular localization of a metabolic component, and biosensors that e.g. measure intracellular $\mathrm{pH}$ or the concentration of a particular metabolite. In research in which the function of a coding sequence (cds) is investigated, the only part of the vector that should change is the cds. In a similar way, promoter functionality studies require a change of promoter for each expression vector while maintaining the reporter gene and terminator that are already present. In many Biobrick systems, all individual elements (promoter, terminator, cds, etc) are integrated in a standard backbone in order to create an expression vector. However, these vectors lack the option to exchange a single element after the vector is constructed.

\section{Need for efficient pathway transfer methods}

In many cases metabolic network redesign requires the introduction of multiple genes or even complete biosynthetic pathways (see e.g. Medema et al. 2014). ${ }^{2}$ However, the use of filamentous fungi as production chassis has major limitations in such cases, as the presently used methods do not accommodate the efficient introduction of multiple genes of large biosynthetic pathways in a single step. When the genes are introduced in a step-by-step manner, it is a disadvantage that every next step requires a new transformation selection marker, implicating that the introduction of the various genes is limited by the number of available selection markers. In practice, this implies that only four to six genes can be introduced into the fungal chassis in such a step-by-step procedure. The limited availability of selection markers is circumvented through recycling the selection markers as shown by several groups. ${ }^{3,4,5}$ But even if recycling of the marker can be achieved, the step-by-step introduction of multiple genes is very time consuming. 


\section{Itaconic acid production in $A$. niger by efficient pathway transfer}

One of the filamentous fungi that is commonly used in industry for the production of food ingredients, pharmaceuticals and industrial enzymes is Aspergillus niger. ${ }^{6,7}$ A. niger has been applied as a chassis for the production of citric acid for decades. The worldwide production of citric acid using $A$. niger is estimated to exceed a million tons a year. ${ }^{8}$ Both the host chassis and the fermentation process have been highly optimized over the years, resulting in an extremely efficient process. Itaconic acid is a direct metabolic derivative of citric acid and an attractive compound for the production of synthetic polymers. ${ }^{9}$ In addition, it is used as a bioactive compound in agriculture, pharmacy and as a medicine. ${ }^{10}$ Currently, itaconic acid is being produced in a fermentative process using Aspergillus terreus reaching production levels of about $80 \mathrm{~g} / \mathrm{L} .{ }^{11}$ Given the high titers for citric acid in A. niger, reported to be over $200 \mathrm{~g} / \mathrm{L}^{8}$, theoretical itaconic acid titers of about $150 \mathrm{~g} / \mathrm{L}$ in $A$. niger should be feasible, making this organism an attractive chassis for the production of itaconic acid.

In A. terreus, itaconic acid is produced from cis-aconitate by the action of cis-aconitate decarboxylase (CadA) (Fig. 5.1).

In $A$. niger this enzyme is lacking, but introduction of the $A$. terreus cadA gene resulted in the production of low amounts of itaconic acid. ${ }^{12}$

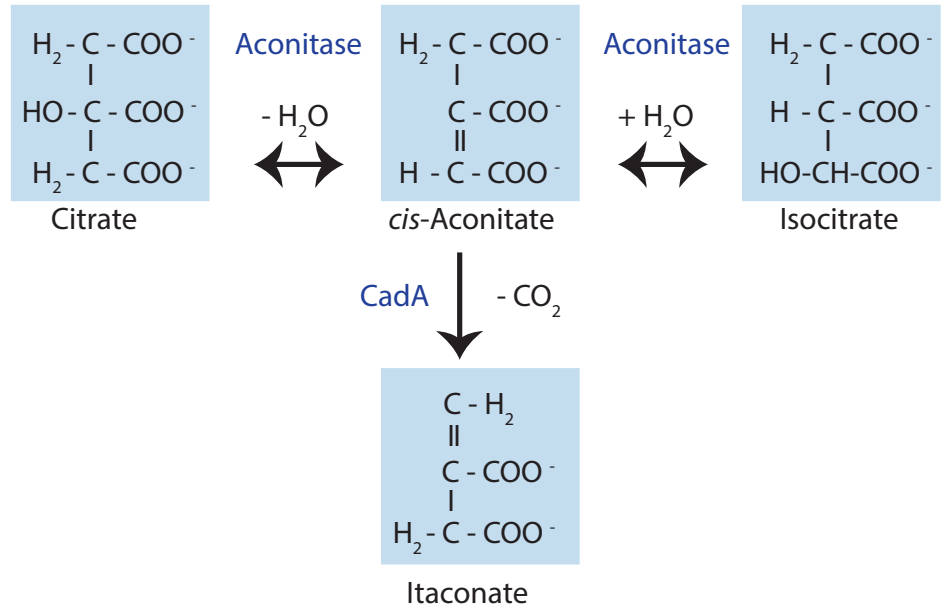

Figure 5.1 Metabolic reactions in A. terreus that lead to the synthesis of itaconate from citrate via cis-aconitate. The reactions are catalysed by aconitase and cis-aconitate decarboxylase (CadA). ${ }^{13}$

The introduction of the $A$. terreus putative mitochondrial transporter $m t t A$ and the plasma membane transporter $m f s A$ in $A$. niger have been shown to greatly enhance itaconic acid titers. ${ }^{14}$ Nevertheless, these itaconic acid titers are still relatively low as compared to the theoretical maximum. This may result from the cytosolic localization of CadA, while its substrate is generated in the mitochondria. Over the years there has been dispute about 
the cellular components of the itaconic acid metabolic network, but for A. terreus the CadA cellular localization was determined to be cytosolic. ${ }^{15}$

In this context, $A$. niger seems to form the perfect chassis for an efficient one-step transfer of the $A$. terreus genes (cadA, $m t t A$ and $m f s A$ ) to allow the fermentative production of itaconic acid, and study the localization of the involved enzyme and transporters. To that end, we designed a vector in which the different elements like promoter, coding sequence, terminator and selection marker can be changed without the need of rebuilding the complete vector.

In this study we report the design of the system, which we termed Funbrick (a contraction of Fungal and Biobrick), the use of this system for the introduction of the itaconic acid biosynthesis genes in A. niger, the subsequent itaconic acid production titers, and the use of a reporter device to show the localization of the itaconic acid biosynthesis genes.

\section{Materials and methods}

\section{Design of Funbricks}

The basic Funbrick (Fig. 5.2) is designed in a way that each functional part is flanked by unique restriction sites.

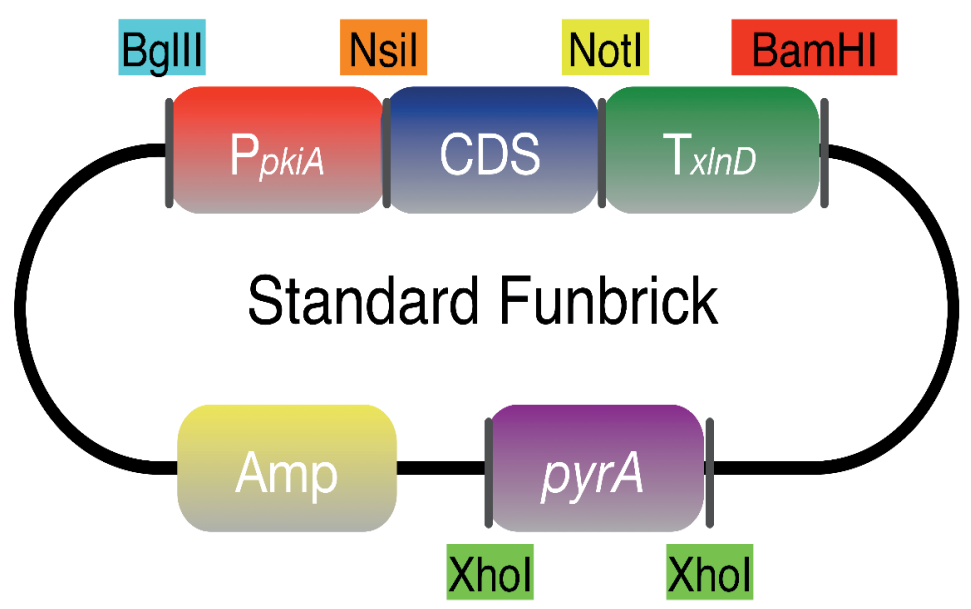

Figure 5.2 Basic Funbrick design.

The promoter sequence (red) is flanked by Bglll and Nsil restriction sites. The coding sequence (blue) is flanked by restriction sites Nsil and Notl. The terminator sequence (green) is flanked by restriction sites Notl and BamHI. The fungal selection marker (purple) is flanked by two Xhol sites. The beta-lactamase (b/a) gene responsible for the ampicillin resistance is depicted in yellow.

The promoter, terminator and coding sequences are flanked by unique restriction sites. This design enables the easy and quick exchange of parts within the expression vector. The fungal selection marker is flanked by Xhol sites as the orientation of the selection marker 
on the plasmid is not important for its functioning. The tandem cloning strategy makes use of the fact that Bglll and BamHI digestions result in compatible sites. Through linearizing the first Funbrick by digestion with BamHI, and digesting the second Funbrick with BamHI and $B g / I I$, the expression cassette of Funbrick 2 can be ligated into Funbrick 1, resulting in a mixed site between the two expression cassettes. So, by using this strategy the structure of the Funbrick will stay intact and the procedure can be repeated until the maximum size of the plasmid is reached.

\section{Itaconic acid biosynthesis constructs}

Standard methods were used to carry out DNA manipulations and E. coli transformations. ${ }^{16}$ All plasmids were propagated in $\mathrm{DH} 5 \alpha$ E. coli. Medium used for E. coli growth was LB supplemented with appropriate antibiotics (ampicillin $100 \mathrm{mg} / \mathrm{L}$ ). The codon optimized genes $m t t A$ and $m f s A$ were synthesized by DNA 2.0 (Menlo Park, USA) and cloned in a pUC19 derived vector under control of a modified $x \ln D$ promoter and the terminator of the $x \ln D$ gene of $A$. niger ${ }^{17}$ resulting in plasmids PMTT and pMFS. The codon optimized gene cadA was synthesized by Baseclear (Leiden, The Netherlands) and also cloned in a pUC19 derived vector under control of the modified $x \ln D$ promoter and $x \ln D$ terminator of A. niger resulting in plasmid pCADs. A Bglll restriction site was introduced in plasmid pMTT using the QuikChange Lightning Site-Directed Mutagenesis Kit (Agilent) using primers LS_ pMTT+BgIII_sense and LS_pMTT+BgIII_antisense. The mutation was confirmed by restriction analysis with Bglll as well as by sequencing and the plasmid was renamed pLS002. An Xhol restriction site was introduced using the same kit using primers LS_PMTT+Xhol_sense and LS_PMTT+Xhol_antisense. The mutation was confirmed by restriction analysis with Xhol as well as by sequencing and the plasmid was renamed pFB001. The $m f s A$ gene contained an Xhol restriction site, which was removed by a silent mutation using primers LS_pMFSXhol_sense and LS_pMFS-Xhol_antisense.

Also this mutation was confirmed using restriction analysis with Xhol and sequencing of the plasmid, which was renamed pLS003. The coding sequence of $\mathrm{mfS} A$ and cadA were obtained by digesting plasmids pLS003 and pCAD with Nsil and Notl and ligated into pFB001 digested with Nsil and Notl. This resulted in plasmids pFB003 and pFB002, respectively. In order to obtain a plasmid that contains all three genes the BgIII-pxInD*-mfsA-txInD-BamHI fragment was obtained by digesting pFB003 with $\mathrm{Bg} / \mathrm{ll}$ and $\mathrm{BamHI}$ and ligated into pFB001 digested with BamHI. This resulted in plasmid pFB004 containing the expression cassettes of both $m t t A$ and $m f s A$ transporters. The BgllI-px/nD*-cadA-tx/nD-BamHI fragment was obtained by digesting pFBOO2 with $\mathrm{Bg} / \mathrm{ll}$ and $\mathrm{BamHI}$ and ligated into pFBOO4 digested with BamHI. This resulted in plasmid pFB005 containing the expression cassettes for all three genes from the itaconic acid biosynthesis cluster of $A$. terreus. An overview of the steps involved is given in Fig. 5.3. 


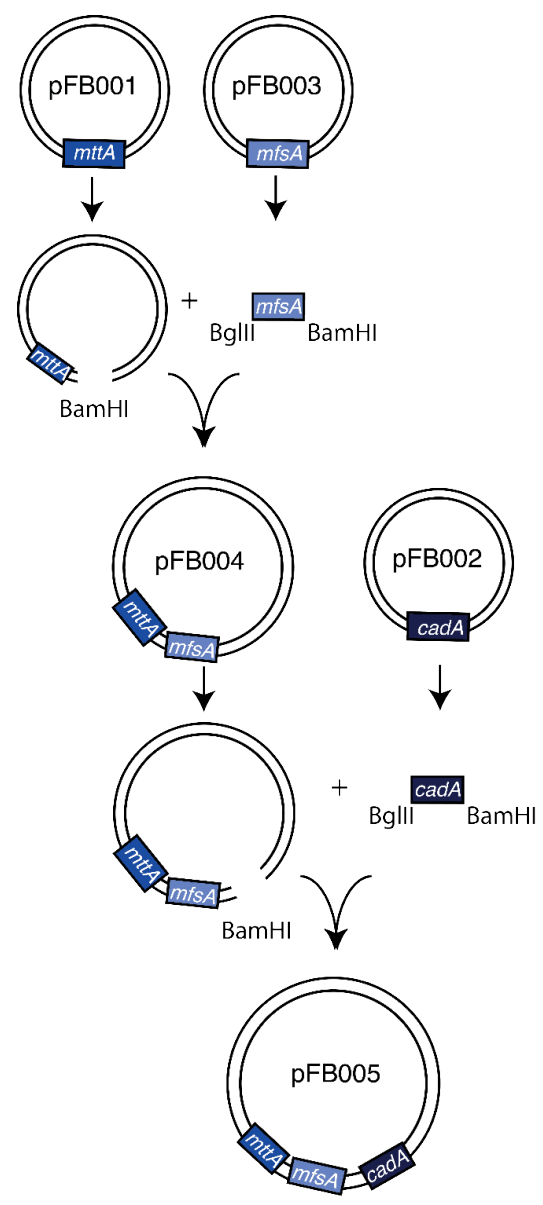

Figure 5.3 Tandem cloning strategy.

Schematic overview of the tandem cloning strategy of subsequent digestion and ligation steps to obtain a Funbrick containing the itaconic acid biosynthesis cluster.

The selection marker pyrA ${ }^{18}$ was synthesized by Baseclear (Leiden, The Netherlands) and cloned into pFB001 digested with Xhol resulting in pFB107. The Xhol-pyrA-Xhol fragment was obtained by digesting pFB107 with Xhol and ligated into pFB005 linearized with Xhol resulting in plasmid pFB303. Due to the relatively large size of the plasmid the transformed E. coli was grown on LB supplemented with $25 \mathrm{mg} / \mathrm{L}$ ampicillin.

\section{Localization constructs}

The localization vectors were made by introducing enhanced GFP sequences at the $\mathrm{N}$-terminal end or the $\mathrm{C}$-terminal end of the coding sequence. A schematic representation of the construction of the N-terminal GFP expression vector is given in Fig. 5.4.

As a basis for the construction of the localization vector plasmid pFB107 was used containing the selection marker for fungal transformations. The N-terminal GFP construct was made 
using restriction enzymes $\mathrm{Nsil}$ and $\mathrm{Pst}$, which have compatible ends after digestion. After ligation the mixed site Nsil/Pst/ will result in a scar. Obviously, the N-terminal GFP does not have a stop codon to ensure that the gene of interest is attached to the GFP molecule. The construction of the C-terminal GFP expression vector was done in a similar way, using Notl and Eag/ restriction enzymes.

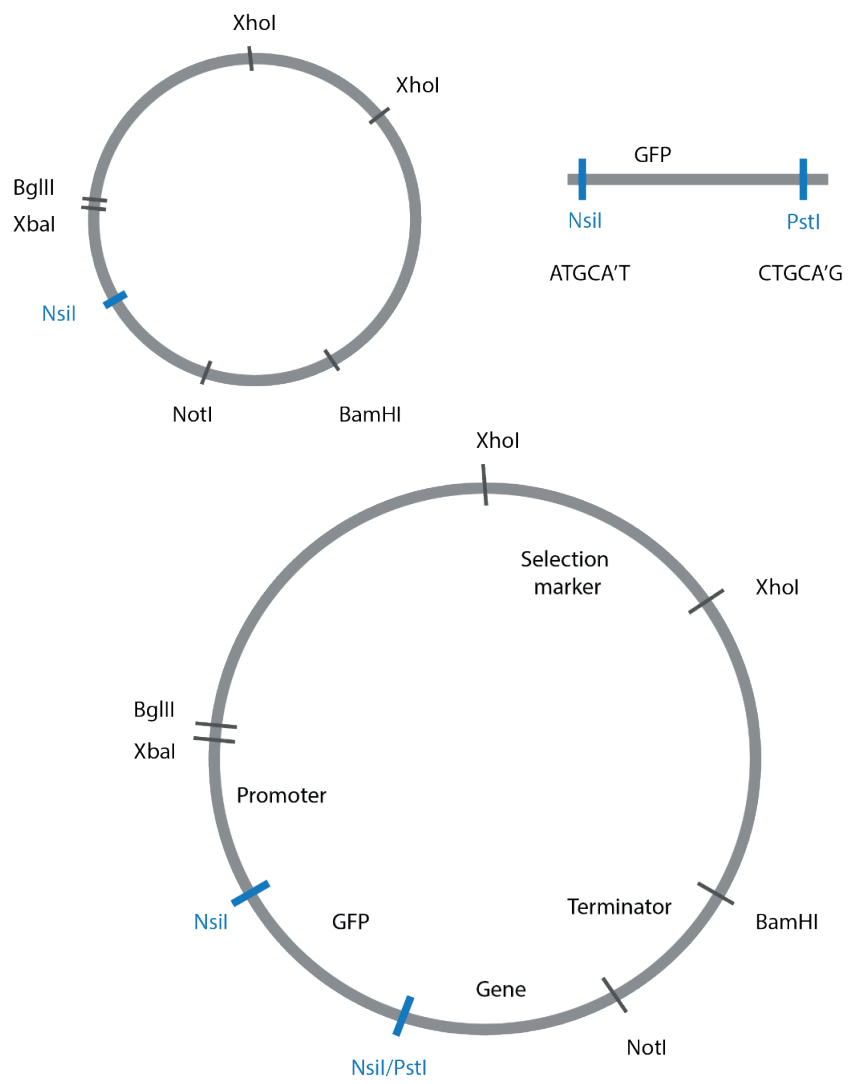

Figure $\mathbf{5 . 4}$ Construction of GFP expression vector.

Schematic overview of the construction of the N-terminal GFP expression vector usable for localization of MttA, MfsA and CadA in A. niger.

For the localization of the itaconic acid biosynthesis cluster genes cadA, $m t t A$ and $m f s A$ the plasmids containing the genes were digested with Nsil and Notl and ligated into the $\mathrm{N}$-terminal and C-terminal GFP expression vectors. For the deletion of the stopcodon a QuikChange Lightning Site-Directed mutagenesis kit

was used (Agilent). Primers were designed according to the instructions of the kit, the design was such that no frame shift occurs (Table 1). For the removal of the stopcodon between the $m$ ttA gene and the GFP gene primers LS_MttA-GFP-no-stop and LS_MttA-GFPno-stop_anti were used. 
Primers LS_CadA-GFP-no-stop and LS_CadA-GFP-no-stop_anti were used to delete the stop codon of the cadA gene and primers LS_MfSA-GFP-no-stop and LS_MfsA-GFP-no-stop_anti were used to delete the stop codon of the $m f s A$ gene. The final N-terminal GFP localization plasmids were named pFB300 containing the mttA gene, pFB301 containing $m f s A$ and pFB302 containing the cadA gene. The final C-terminal GFP localization plasmids were named pFB308 containing the $m t t A$ gene, pFB309 containing the $m f s A$ gene and pFB310 containing cadA.

\section{Strain and spore preparation}

The fungal strain used in this study was A. niger NW186 (cspA1, goxC17, prtF28 $\triangle \arg B$, pyrA6), which is a pyrA mutant of $A$. niger NW185. ${ }^{19}$

\section{Fungal transformation}

Protoplasts were generated using Novozyme-234 for the transformation of the A. niger NW186 strain. For the transformation of A. niger NW186::pFB303, protoplasts were generated using Vinotaste. The pFB303 plasmid was introduced in A. niger NW186 by transformation as described before. ${ }^{20}$ The pFB002 plasmid was introduced in $A$. niger NW186::pFB303-1 by co-transformation using the pAL69 plasmid, which contains the argB gene. Selective MMS plates $\left(6.0 \mathrm{~g} / \mathrm{L} \mathrm{NaNO}_{3}, 1.5 \mathrm{~g} / \mathrm{L} \mathrm{KH}_{2} \mathrm{PO}_{4}, 0.5 \mathrm{~g} / \mathrm{L} \mathrm{KCl}, 0.5 \mathrm{~g} / \mathrm{L} \mathrm{MgSO}{ }_{4} 7 \mathrm{H}_{2} \mathrm{O}\right.$, $1 \mathrm{~mL} / \mathrm{L}$ Vishniac solution ${ }^{21}$, $325.2 \mathrm{~g} / \mathrm{L}$ sucrose, $1.2 \%$ (w/v) agar, $\mathrm{pH}$ 6.0) were used to select for protoplasts that integrated the plasmid containing the $p y r A$ selection marker or the $\arg B$ selection marker. Colonies were randomly picked from the transformation plates and replated on complete medium. ${ }^{22}$ Plasmids pFB308, pFB309 and pFB310 were introduced in A. niger NW186 by direct transformation using $1 \mu \mathrm{g}$ of DNA.

\section{DNA extraction and PCR}

Fresh mycelium was disrupted using Fastprep with glass beads and $400 \mu \mathrm{L}$ DNA extraction buffer (0.1 M Tris $\mathrm{HCl}$ pH 8.0, 1.2 M NaCl, 5 mM EDTA). DNA was extracted using phenolchloroform extraction. The pellet was washed with $70 \%$ cold ethanol, air-dried and resuspended in $50 \mu \mathrm{L}$ Milli-Q water. Transformants were identified by PCR using the extracted genomic DNA as a template.

\section{Growth experiments and metabolite analysis using HPLC}

The different $A$. niger transformants and the control strain were grown, after inoculation of $10^{6}$ spores per $\mathrm{mL}$, in $1 \mathrm{~L}$ Erlenmeyer flasks containing $200 \mathrm{~mL} \mathrm{PM}$ medium ${ }^{19}$ (1.2 g NaNO${ }_{3^{\prime}}$ $0.5 \mathrm{~g} \mathrm{KH}_{2} \mathrm{PO}_{4^{\prime}} 0.2 \mathrm{~g} \mathrm{MgSO}_{4} 7 \mathrm{H}_{2} \mathrm{O}, 0.5 \mathrm{~g}$ yeast extract and $40 \mu \mathrm{L}$ Vishniac per liter) ${ }^{21}$ with 100 $\mathrm{mM}$ sorbitol as a carbon source. The expression of the different recombinant genes studied in this work was induced, $18 \mathrm{~h}$ after inoculation ( $\mathrm{t}=0$ ), with $50 \mathrm{mM}$ xylose. All $A$. niger strains were grown for 5 days at $30^{\circ} \mathrm{C}$ and $250 \mathrm{rpm}$. Samples for HPLC analysis were taken at $0 \mathrm{~h}$, $6 \mathrm{~h}, 30 \mathrm{~h}, 54 \mathrm{~h}$ and $78 \mathrm{~h}$ after induction. 
To obtain the acid concentrations, a Bio-Rad Aminex HPX-87H column was used and eluted with $4 \mathrm{mM} \mathrm{H}_{2} \mathrm{SO}_{4}$ at a flow rate of $0.6 \mathrm{~mL} / \mathrm{min}$. Sampling was done at $35^{\circ} \mathrm{C}$ for $30 \mathrm{~min}$. Detection was done by both a refractive index detector (Shodex RI-101, sample frequency $10 \mathrm{~Hz}$ ) and a UV-VIS detector (Dionex ICS VWD, $\lambda=210 \mathrm{~nm}$ ). Propionic acid $(6 \mathrm{mM})$ was used as an internal standard. To obtain the sugar concentrations, a Dionex MA-1 column was used and eluted with $600 \mathrm{mM} \mathrm{NaOH}$ at a flow rate of $0.4 \mathrm{~mL} / \mathrm{min}$. Sampling was done at $25^{\circ} \mathrm{C}$ for $23 \mathrm{~min}$. Detection was done by electrochemical detection (Dionex ICS EDet1, waveform, gold standard PAD quadratic potential).

\section{RNA isolation, cDNA synthesis and $q P C R$}

Isolation of RNA, preparation of cDNA, and qPCR analysis were done as described previously. ${ }^{23}$

\section{Microscopy}

Fresh spores were harvested at the day before the microscopy experiment. 250 spores were inoculated in $200 \mu \mathrm{L}$ minimal medium containing $10 \mathrm{mM}$ sorbitol and grown overnight at $30{ }^{\circ} \mathrm{C}$. Two hours before making the microscopic pictures the cells were induced by the addition of $10 \mathrm{mM}$ D-xylose. Mitotracker (ThermoFisher Scientific) was used for co-localization of the mitochondria. Shortly before making the pictures $20 \mathrm{nM}$ mitotracker was added to the sample.

\section{Results and Discussion}

In this research we designed and constructed a standard Funbrick (Fig. 5.2), which allowed a fast and efficient way to construct several Funbricks for expression of the itaconic acid biosynthesis genes, cadA, mttA and $m f S A$ into the $A$. niger genome in one transformation event. Expression of these three genes in $A$. niger was confirmed using qPCR. In addition, vectors were constructed for the localization of $c a d A, m t t A$ and $m f s A$ genes in a similar manner.

\section{Itaconic acid production levels in $A$. niger}

After successfully introducing the cadA, $m t t A$ and $m f s A$ genes in $A$. niger, the production levels of various metabolites were measured using HPLC (Fig 5.5).

Expression of these genes resulted in itaconic acid production in three out of four transformants, albeit at relatively low concentrations. The highest itaconic acid concentration that was reached was $169 \mathrm{mg} / \mathrm{L}, 78 \mathrm{~h}$ after induction. The production of citric acid varied between $0.2 \mathrm{~g} / \mathrm{L}$ for transformant 2 and $1.6 \mathrm{~g} / \mathrm{L}$ for transformant 3, compared to $1.0 \mathrm{~g} / \mathrm{L}$ for the control strain. Surprisingly, fairly high concentrations of cis-aconitic acid were detected in the transformants. At $78 \mathrm{~h}$ after induction concentrations of cis-aconitic acid were found between $0.8 \mathrm{~g} / \mathrm{L}$ and 2.2 $\mathrm{g} / \mathrm{L}$. No cis-aconitic acid was detected in the supernatant of the control strain. Also no succinic acid, malic acid, pyruvic acid or $\alpha$-ketoglutaric acid was detected in the samples. 
These results show that by expressing the cadA, $m t t A$ and $m f s A$ genes in a stoichiometric ratio, the production of itaconic acid is far from efficient.

Apparently, there is not enough capacity of CadA in the cell to convert cis-aconitic acid into itaconic acid since the main acid secreted in three of the four transformants is cis-aconitic acid. cis-Aconitic acid is actually an intermediate in the isomerization reaction of citrate to isocitrate, catalyzed by aconitase.

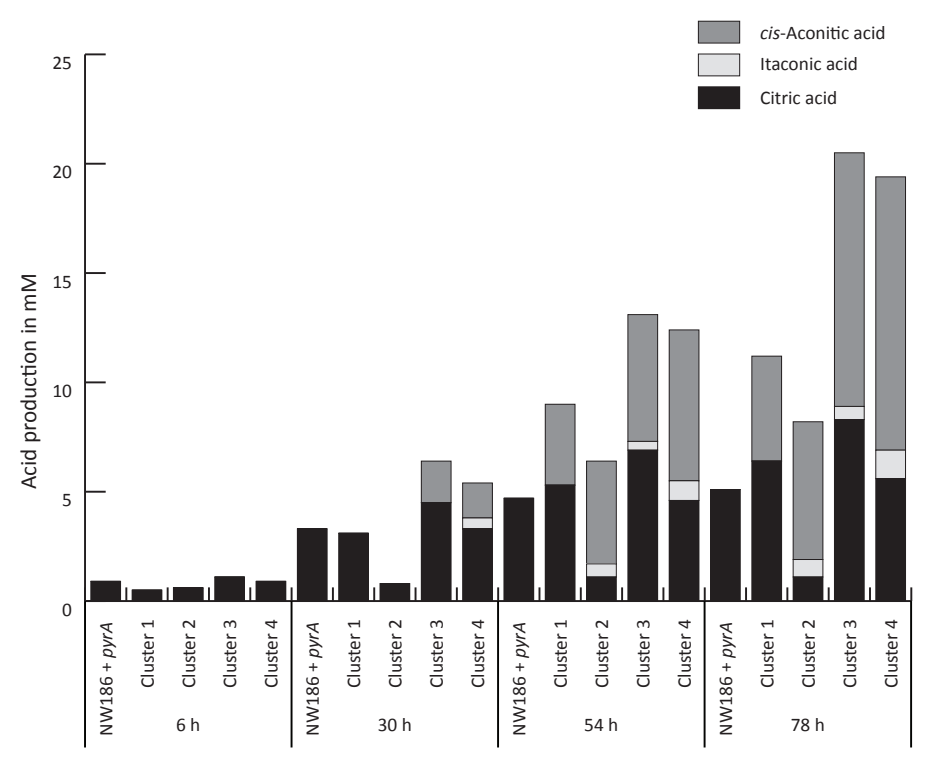

Figure 5.5 Production of cis-aconitic acid, itaconic acid and citric acid in time by the four transformants compared with the parent strain transformed with an empty vector.

A possible explanation for the presence of cis-aconitic acid in the medium is that the mitochondrial transporter $m t t A$ is actually transporting cis-aconitic acid from the mitochondrium into the cytosol where it accumulates. Subsequently, it is exported outside the cell, possibly facilitated by the membrane transporter $m f s A$.

Previously published data on itaconic acid production in $A$. niger showed that it is possible to produce itaconic acid quite efficiently by expressing the three genes from $A$. terreus cadA, $m t t A$ and $m f s A$ in a step-by-step approach (Chapter $2,{ }^{14}$ ). These strains reached itaconic acid production levels between $5.5 \mathrm{~g} / \mathrm{L}$ and $7.1 \mathrm{~g} / \mathrm{L}$ using the same growth conditions.

In the step-by-step approach, the cadA gene first was introduced by co-transformation, which resulted in similar itaconic acid production levels $(0.3 \mathrm{~g} / \mathrm{L})$ as we found in our strains that express all three genes. The highest itaconic acid producing cadA expression strain was subsequently transformed with both transporters resulting in the 5.5-7.1 g/L production levels (Chapter $2,{ }^{14}$ ). A co-transformation generally leads to more copies of the cadA gene than a direct transformation as performed in this study. So, where in the previous study the 
lack of transport caused the limited production of itaconic acid, in this study the limiting step is probably the CadA activity. This was investigated by introducing extra copies of the cadA gene in transformant NW186::pFB303-1. In the absence of extra copies of the cadA gene, this particular transformant did not produce any itaconic acid. After co-transformation with the plasmid containing the cadA gene, this strain produced $5.0 \mathrm{~g} / \mathrm{L}$ itaconic acid after $78 \mathrm{~h}$ of growth which confirmed the hypothesis that the reaction catalyzed by CadA was the limiting step. In order to create an efficient itaconic acid production process in $A$. niger, the ratio of expression between $c a d A, m t t A$ and $m f s A$ is important to optimize. Therefore, to improve the production of itaconic acid, a solution is to perform co-transformations instead of direct transformations. Another possibility is, for instance, to use different promoters of different strengths using the Funbrick system. We showed in this research that it is indeed easy and fast to work with the Funbrick system but more improvents should be made in order to achieve industrial production levels.

\section{Localization of cadA, $m t t A$ and $m f s A$ in Aspergillus niger}

The localization vectors were made in such a way that the genes that were studied using the expression vectors could easily be cloned in the localization vector. Only the C-terminal expression vector needs a two-step approach since part of the stop codon of the gene of interest has to be removed in order to ensure a correct translation process.

For the three different plasmids pFB308, pFB309 and pFB310, containing the $m t t A, m f s A$ and cadA gene, respectively, several transformants were obtained. A microscopic image of a transformant expressing the $m t t A$ gene is shown in Fig. 5.6. MttA appears to be located in the mitochondria, which was recently confirmed by Steiger et al. (2016). ${ }^{24}$

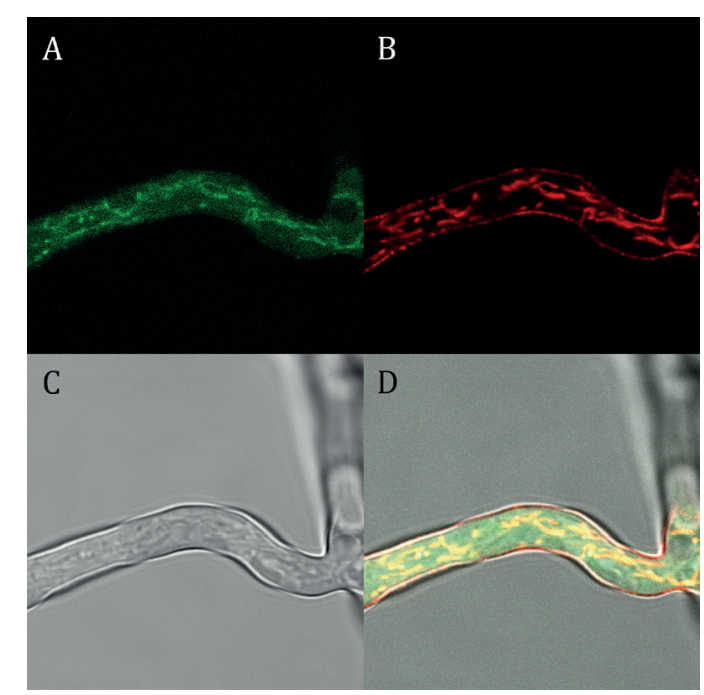

Figure 5.6 Localization of MttA.

MttA localization (green) in A. niger NW186 transformed with plasmid pFB308. The mitochondria are stained with mitotracker (red). A: green fluorescent signal; B: red fluorescent signal; C: no signal; D: overlay. 
Fig. 5.7 shows the transformant expressing the $m f s A$ gene. MfsA appears neither to be located in the mitochondria nor in the cytosol but possibly in vacuoles or other vesicle-like structures. CadA is clearly located in the cytosol, as can be seen in Fig. 5.8. These findings are in line with Jakltisch et al. where they found the CadA activity solely in the cytosol. ${ }^{15}$

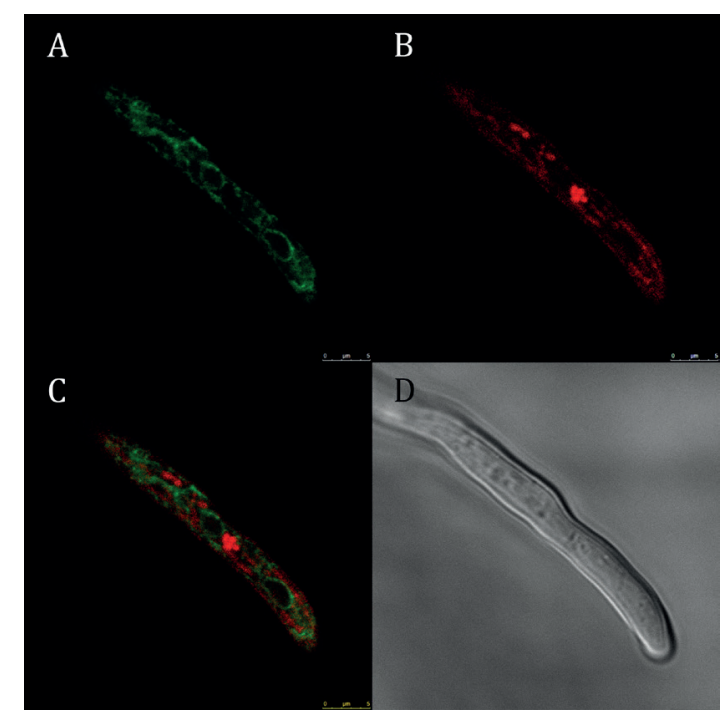

Figure 5.7 Localization of MfsA.

MfsA localization (green) in A. niger NW186 transformed with plasmid pFB309. The mitochondria are stained with mitotracker (red). A: green fluorescent signal; B: red fluorescent signal; C: no signal; D: overlay.

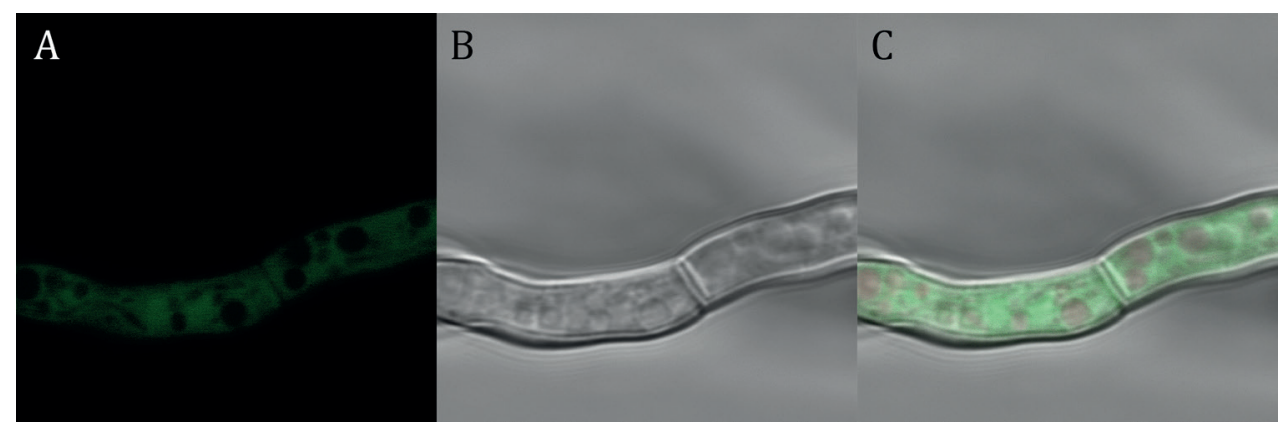

Figure 5.8 Localization of CadA.

CadA localization (green) in A. niger NW186 transformed with plasmid pFB310. A: green fluorescent signal; B: no signal; C: overlay. 


\section{Conclusions}

In this research we showed that the newly designed Funbrick system, composed of standardized expression vectors and localization vectors, was successfully applied to express and localize the itaconic acid biosynthesis cluster in A. niger. For future purposes this system can easily be used to study the function of various genetic elements such as promoters, terminators and selection markers. The Funbrick system can also be extended further in order to express large clusters of genes by using cosmids. Use of cosmid vectors in transformation of filamentous fungi has been described previously. However, their use is restricted to construct libraries for e.g. complementation of mutations ${ }^{25}$ or for genome walking experiments. ${ }^{26}$

\section{Competing interests}

The authors declare that they have no competing interests.

\section{Authors' contributions}

LvdS designed and performed the experimental work and participated in writing the manuscript. RH and NB designed and performed the experimental work. LdG designed the study and participated in writing of the manuscript.

\section{Acknowledgements}

We thank Mark van Passel for helpful discussions in the initial phase of the design of the Funbrick system. We would also like to thank Douwe van der Veen for the helpful discussions about the qPCR work. This work has been carried out on the basis of a grant in the framework of the BE BASIC program F01.002 itaconic/fumaric acids: Novel economic and eco-efficient processes for the production of itaconic and fumaric acid. 


\section{Supplementary information}

Table 1 Primers used in this study

\begin{tabular}{|c|c|}
\hline Name of primer & Sequence $5^{\prime}-3^{\prime}$ \\
\hline LS_pMTT+BgIII_sense & AGCTTGCATGCCTGCAGATCTACTCTAGACGAATGAGG \\
\hline LS_pMTT+BgIII_antisense & СCTCATTCGTCTAGAGTAGATCTGCAGGCATGCAAGCT \\
\hline LS_pMTT+Xhol_sense & GAGACGAAAGGGCCTCGAGATACGCCTATTTTTAT \\
\hline LS_pMTT+Xhol_antisense & ATAAAAATAGGCGTATCTCGAGGCCCTTTCGTCTC \\
\hline LS_pMFS-Xhol_sense & CCCTTGCACCTGCTTGAGACGAAGTGCAG \\
\hline LS_pMFS-Xhol_antisense & CTGCACTTCGTCTCAAGCAGGTGCAAGGG \\
\hline LS_MttA-GFP-no-stop & GTTCTTGACCCAACCTAACAGCGGCCGCATG \\
\hline LS_MttA-GFP-no-stop_anti & CATGCGGCCGCTGTTAGGTTGGGTCAAGAAC \\
\hline LS_CadA-GFP-no-stop & TCGCCACTAGTGGGCGGCCGCATG \\
\hline LS_CadA-GFP-no-stop_anti & CATGCGGCCGCCCACTAGTGGCGA \\
\hline LS_MfsA-GFP-no-stop & CTGCCGACACAGAGCGGCCGCATG \\
\hline LS_MfsA-GFP-no-stop_anti & CATGCGGCCGCTCTGTGTCGGCAG \\
\hline LS_qCAD_F & ACTCCGAAGAGTTCGACCAG \\
\hline LS_qCAD_R & ACCAGGTCCTCGATTTCCTT \\
\hline LS_qMTT_F & ATTAAGACCCGCATGCAATC \\
\hline LS_qMTT_R & CTTCTCGTAGACGGGGAACA \\
\hline LS_qMFS_F & TTCTTGATCGGCTCTCTGGT \\
\hline LS_qMFS_R & GAGGGTCCAGCCATAAATCA \\
\hline LS_qkan_F & AGCATTACGCTGACTTGACG \\
\hline LS_qkan_R & AGGTGGACCAGTTGGTGATT \\
\hline LS_qAn08g06940_F & ATCTTGCGTGACAACATCCA \\
\hline LS_qAn08g06940_R & CACCCTCAAGGAAGGTCTTG \\
\hline LS_qAn02g04120_F & TTTTCAGTCTGGCTGCTCCT \\
\hline LS_qAn02g04120_R & CTGTTTTCCTGCATCGTGTG \\
\hline
\end{tabular}




\section{References}

1. Lubertozzi D, Keasling JD. Developing Aspergillus as a host for heterologous expression. Biotechnol Adv 2009, 27:53-75.

2. Medema MH, Alam MT, Breitling R, Takano E. The future of industrial antibiotic production: From random mutagenesis to synthetic biology. Bioengineered Bugs 2014,2:230-233.

3. Zhang D-X, Lu H-L, Liao X, St. Leger RJ, Nuss DL. Simple and efficient recycling of fungal selectable marker genes with the Cre-loxP recombination system via anastomosis. Fungal Genet Biol 2013,61:1-8.

4. Kopke K, Hoff B, Kuck U. Application of the Saccharomyces cerevisiae FLP/FRT recombination system in filamentous fungi for marker recycling and construction of knockout strains devoid of heterologous genes. Appl Environ Microbiol 2010,76:4664-4674.

5. Delmas S, Llanos A, Parrou J-L, Kokolski M, Pullan ST, Shunburne L et al. Development of an unmarked gene deletion system for the filamentous fungi Aspergillus niger and Talaromyces versatilis. Appl Environ Microbiol 2014,80:3484-3487.

6. Pel HJ, de Winde J, Archer DB, Dyer PS, Hofmann G, Schaap PJ et al. Genome sequencing and analysis of the versatile cell factory Aspergillus niger CBS 513.88. Nature Biotechnol 2007,25:221-231.

7. Punt PJ, van Biezen N, Conesa A, Albers A, Mangnus J, van den Hondel C. Filamentous fungi as cell factories for heterologous protein production. Trends Biotechnol 2002, 20:200-206.

8. Magnuson J, Lasure L. Organic acid production by filamentous fungi. In Advances in Fungal Biotechnology for Industry, Agriculture and Medicine. Edited by Tkacz JS, Lange L. New York: Kluwer Academic/Plenum Publishers; 2004:307-340.

9. Kubicek CP, Punt PJ, Visser J. Production of organic acids by filamentous fungi. The Mycota. Industrial Applications, Edited by M. Hofrichter, Berlin, Heidelberg: Springer Berlin Heidelberg. 2010:215-234

10. Okabe M, Lies D, Kanamasa S, Park EY. Biotechnological production of itaconic acid and its biosynthesis in Aspergillus terreus. Appl Microbiol Biotechnol 2009,84:597-606.

11. Kuenz A, Gallenmüller Y, Willke T, Vorlop K-D. Microbial production of itaconic acid: developing a stable platform for high product concentrations. App/ Microbiol Biotechnol 2012,96:1209-1216.

12. Li A, van Luijk N, ter Beek M, Caspers M, Punt P, van der Werf M. A clone-based transcriptomics approach for the identification of genes relevant for itaconic acid production in Aspergillus. Fungal Genet Biol 2011,48:602-611.

13. Dwiarti L, Yamane K, Yamatani H, Kahar P, Okabe M. Purification and characterization of cisaconitic acid decarboxylase from Aspergillus terreus TN484-M1. J Biosci Bioeng 2002, 94:29-33.

14. van der Straat L, Vernooij M, Lammers M, van den Berg WAM, Schonewille T, Cordewener J, et al. Expression of the Aspergillus terreus itaconic acid biosynthesis cluster in Aspergillus niger. Microbial Cell Fact 2014,13:1-9.

15. Jaklitsch WM, Kubicek CP, Scrutton MC. The subcellular organization of itaconate biosynthesis in Aspergillus terreus. J Gen Microbiol 1991,137:533-539. 
16. Sambrook J, Fritsch EF, Maniatis T. Molecular cloning: A Laboratory Manual, (2nd ed.). 1989. Cold Spring Harbor: Cold Spring Harbor Laboratory Press.

17. van Peij NN, Brinkmann J, Vrsanská M, Visser J, de Graaff LH. beta-Xylosidase activity, encoded by $x \ln D$, is essential for complete hydrolysis of xylan by Aspergillus niger but not for induction of the xylanolytic enzyme spectrum. Eur J Biochem 1997,245:164-173.

18. Goosen T, Bloemheuvel G, Gysler C, Bie DA, Broek HWJ, Swart K. Transformation of Aspergillus niger using the homologous orotidine-5'-phosphate-decarboxylase gene. Curr Genet 1987,11:499-503.

19. Ruijter G, van de Vondervoort P. Oxalic acid production by Aspergillus niger: an oxalate-nonproducing mutant produces citric acid at pH 5 and in the presence of manganese. Microbiology 1999,145:2569-2576.

20. Kusters-van Someren MA, Harmsen JA, Kester HC, Visser J. Structure of the Aspergillus niger pelA gene and its expression in Aspergillus niger and Aspergillus nidulans. Curr Genet 1991,20:293-299.

21. Vishniac W, Santer M. The thiobacilli. Bacteriol Rev 1957, 21:195-213.

22. Pontecorvo G, Roper JA, Hemmons LM, MacDonald KD, Bufton AWJ. The genetics of Aspergillus nidulans. Adv Genet 1953,5:141-238.

23. van der Straat L, Tamayo-Ramos JA, Schonewille T, de Graaff LH. Overexpression of a modified 6-phosphofructo-1-kinase results in an increased itaconic acid productivity in Aspergillus niger. AMB Express 2013,3:57.

24. Steiger MG, Punt PJ, Ram AFJ, Mattanovich D, Sauer M. Characterizing MttA as a mitochondrial cis-aconitic acid transporter by metabolic engineering. Metab Eng 2016,35:95-104.

25. Timberlake WE, Boylan MT, Cooley MB, Mirabito PM, O'Hara EB, Willett CE. Rapid identification of mutation-complementing restriction fragments from Aspergillus nidulans cosmids. Exp Mycol 1985,9:351-355.

26. Orbach MJ. A cosmid with a HyR marker for fungal library construction and screening. Gene 1994,150:159-162. 


\section{6}

(9)
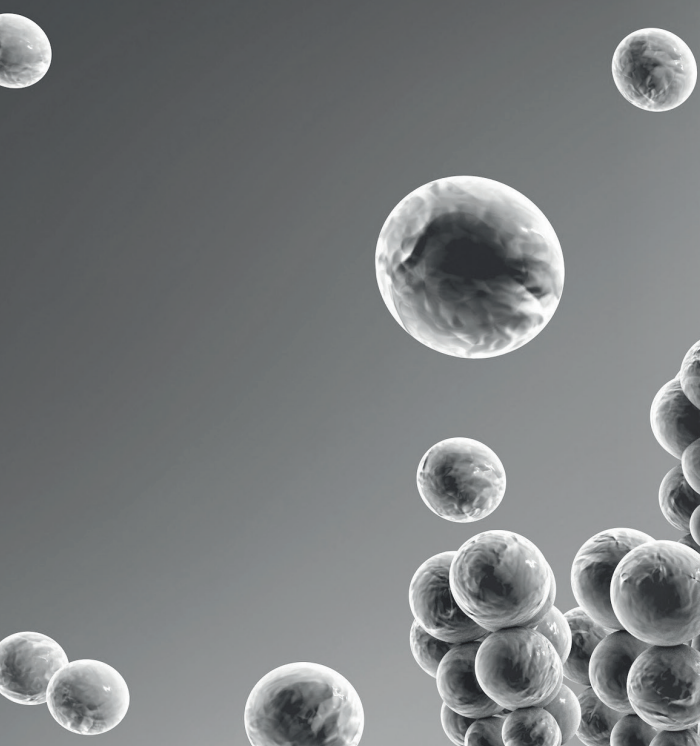

6

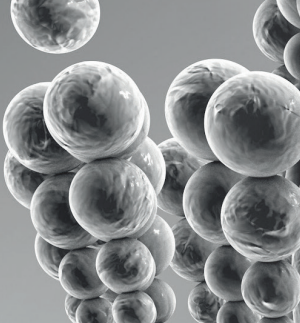

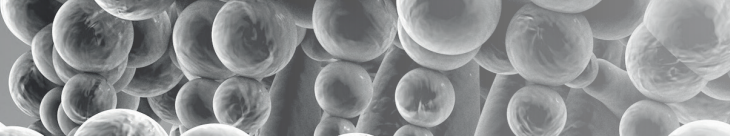
(1)
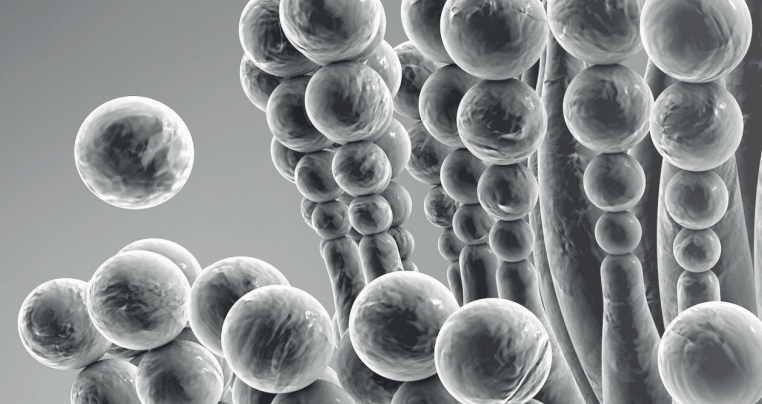

(9)
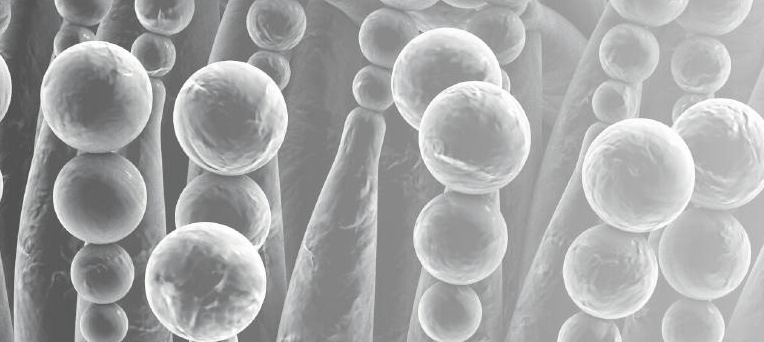

$$
\text { Q }
$$
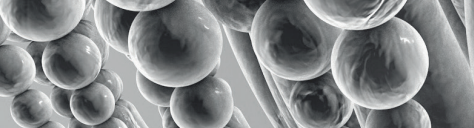

428

init 180

il mo

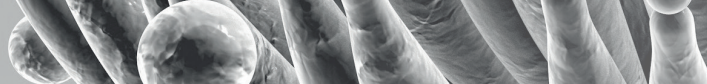

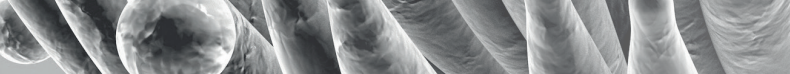

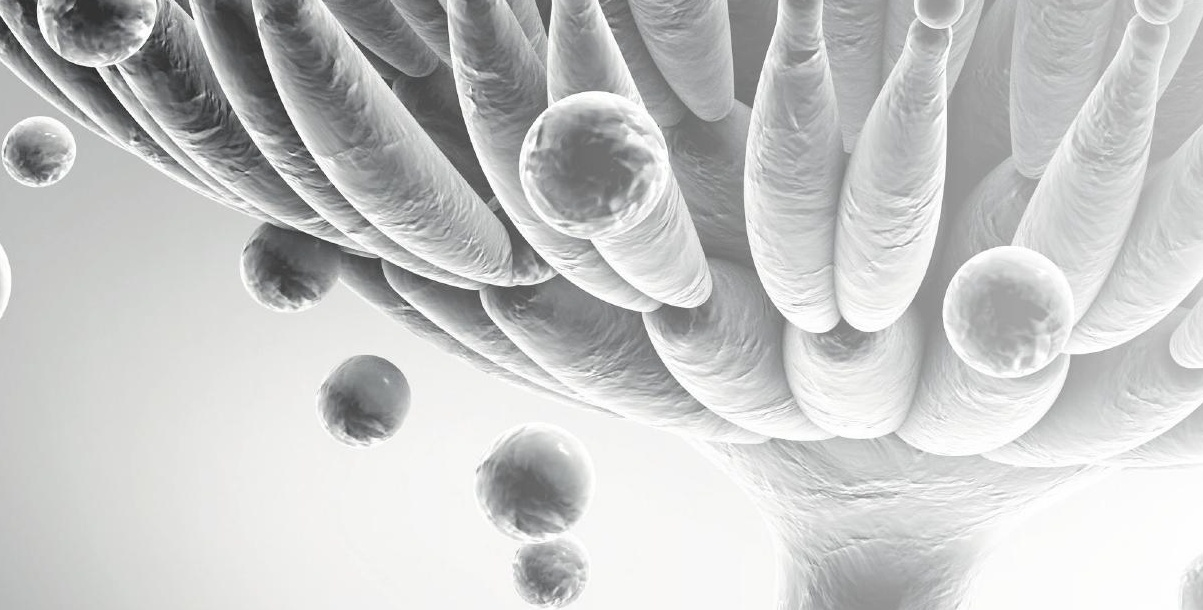

(.

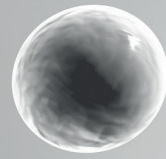

6 


\section{Chapter 6}

\section{Visual selection of targeted integration in Aspergillus niger}




\section{Abstract}

In filamentous fungi, like Aspergillus niger, homologous or targeted integration is relatively rare. The $A$. niger genome encodes a few different genes that are involved in spore color formation. The use of a fragment of one such gene, fwnA, was investigated as a selective marker for homologous integration. Transformants resulting from homologous recombination at the fwnA locus can be distinguished on the basis of their spore color, which is fawn. For some of the transformants the phenotype appeared to be dependent on the osmolarity of the medium used in transformation, and their spore color was grey. Upon purification of these colonies on osmotically non-stabilized minimal medium, the fwnA phenotype appeared. The method described here offers a non-laborious way for the rapid selection of transformants resulting from integration at a defined locus in the genome of $A$. niger, and can be applied to other filamentous fungi as well.

\section{Keywords}

targeted integration, high-throughput screening, strain improvement 


\section{Introduction}

Aspergillus niger has been appreciated as an organism of high interest in biotechnological applications for a long time; as the main workhorse for the production of citric acid and for the production of a variety of industrially important enzymes. ${ }^{1}$ Previously, strain development and strain optimization was mainly done by random mutagenesis followed by laborious screening for the strains having the desired traits. The advent of the genomic era allows for the rational design of genetic modifications. The complete sequence of the genome of $A$. niger has been determined ${ }^{2}$ and the molecular toolbox for this fungus has expanded over recent years. ${ }^{3}$ Although autonomously replicating plasmids do exist in $A$. niger, these are hard to maintain without the application of selective pressure. ${ }^{4} \mathrm{As}$ a result, genetic engineering of $A$. niger has mainly focused on the development of strategies for the integration of genes in the chromosomal DNA. ${ }^{3}$ For such purposes targeted integration is a valuable tool; it allows for the insertion of a DNA fragment of interest in defined regions of the genome, e.g. in highly transcribed genomic areas. This avoids the disruption of other genes as well, and as such avoids genetic background damage.

Recently, a number of genes associated with conidial pigmentation in A. niger have been identified. Among these is a gene that is related to the fwnA phenotype (An09g05730). The gene is homologous to the $W A$ gene of Aspergillus nidulans, which has been shown to play a role in the 1,8-dihydroxynaphthalene (1,8-DHN) pathway; a series of reactions leading to melanin synthesis. ${ }^{5}$ Disruption of this gene leads to fawn (brownish) pigmentation of the spores when the mutants are grown in standard minimal or complete media. The finding that disruption of a single gene can lead to a change in spore color makes this locus a suitable candidate to provide a basis for a targeted integration high-throughput screening method. Specifically, the method is based on the incorporation of fragments of fwnA and subsequent integration of the vector at the fwnA locus that results in two truncated copies of the gene. The fawn colored mutants are straightforwardly distinguishable from their nontransformed counterparts.

\section{Materials and Methods}

\section{Strains}

In this work two auxotrophic $A$. niger strains were used as the parental strains for the generation of the fwnA mutants; the uridine deficient N593 (cspA1, pyrA6) and the uridine/ arginine deficient NW186 (cspA1, $\triangle \operatorname{argB}$ pyrA6 prtF28 goxC17) strain. Both strains are derivatives of $A$. niger N400 (CBS 120.49). 


\section{Construction of plasmids}

PJET1.2/blunt (Fermentas) was used as the backbone for vector construction; four plasmid constructs were made from this vector in this work (Fig. 6.1). Genomic DNA of A. niger N593 ( $\operatorname{csp} A 1$, pyrA6) was used as a template to obtain the fwnA fragments and the $\arg B$ gene. Primers $\mathrm{p} 1$ and $\mathrm{p} 2$ were used to obtain fwnA-part 1, p1 and $\mathrm{p} 3$ were used to obtain fwnApart 2 (Table 6.1). The $\arg B$ gene was obtained using primers $p 4$ and p5 (Table 6.1). The sequence of pyrA ${ }^{6}$ was derived from pGW635 by Xbal digestion.

Table 6.1 Primers used in this study to obtain fwnA fragments and $\arg B$ gene.

\begin{tabular}{ll}
\hline Primer name & Sequence $\mathbf{5}^{\prime} \mathbf{-} \mathbf{3}^{\prime}$ \\
\hline p1 & CTCGAGTGGAGCAACAGCTACAGTCG \\
p2 & GTCGACAGGCTTGGAACTGTGACACC \\
p3 & GTCGACATGTACGGGCAGTTCAATCC \\
p4 & TCTAGAGATATAGATGACGCGGCAAC \\
p5 & TCTAGAGACAATAGTGTCGTCGACAG \\
\hline
\end{tabular}

\section{Transformation of $\boldsymbol{A}$. niger and selective media}

The plasmids were constructed and propagated in E. coli DH5a cells. Transformed cells were plated on LB media containing $100 \mu \mathrm{g} / \mathrm{ml}$ ampicillin and grown overnight at $37^{\circ} \mathrm{C}$. Single colonies were selected and grown for plasmid isolation and plasmid analysis. A. niger protoplasts were generated using Novozyme 234 and used in PEG transformations. 7,8

Strain N593 was transformed using the plasmids pFwnPyr1 and pFwnPyr2, strain NW186 was transformed using plasmids pFwnArg1 and pFwnArg2. A schematic representation of the strategy is presented in Fig. 6.2. The transformed protoplasts were plated in osmotically stabilized minimal medium selective (MMS) plates containing $6 \mathrm{~g} / \mathrm{L} \mathrm{NaNO}_{3}, 1.5 \mathrm{~g} / \mathrm{L} \mathrm{KH}_{2} \mathrm{PO}_{4^{\prime}}$ $0.5 \mathrm{~g} / \mathrm{L} \mathrm{KCl}, 0.5 \mathrm{~g} / \mathrm{L} \mathrm{MgSO}_{4} \cdot 7 \mathrm{H}_{2} \mathrm{O}, 1 \mathrm{~g} / \mathrm{L}$ Vishniac trace elements solution, ${ }^{9} 0.95 \mathrm{M}$ sucrose and $1.2 \%$ agar. Selected colonies were purified in standard MMS plates $(\mathrm{pH} 6)$ containing 6 $\mathrm{g} / \mathrm{L} \mathrm{NaNO}_{3}, 1.5 \mathrm{~g} / \mathrm{L} \mathrm{KH}_{2} \mathrm{PO}_{4}, 0.5 \mathrm{~g} / \mathrm{L} \mathrm{KCl}, 0.5 \mathrm{~g} / \mathrm{L} \mathrm{MgSO} \cdot 7 \mathrm{H}_{2} \mathrm{O}, 1 \mathrm{~g} / \mathrm{L}$ Vishniac trace elements solution, $2 \mathrm{~g} / \mathrm{L}$ casaminoacids, $1 \mathrm{~g} / \mathrm{L}$ yeast extract, $50 \mathrm{mM}$ glucose and $1.5 \%$ agar. 8,10

\section{Results and discussion}

In this study, five plasmids were constructed using two different fungal transformation selection markers and three different fragments of the fwnA gene (Fig. 6.1). These five plasmids were used for the transformation of A. niger strains N593 (pyrA as an selection marker) and NW186 ( $\arg B$ as an selection marker) (Fig. 6.2). 


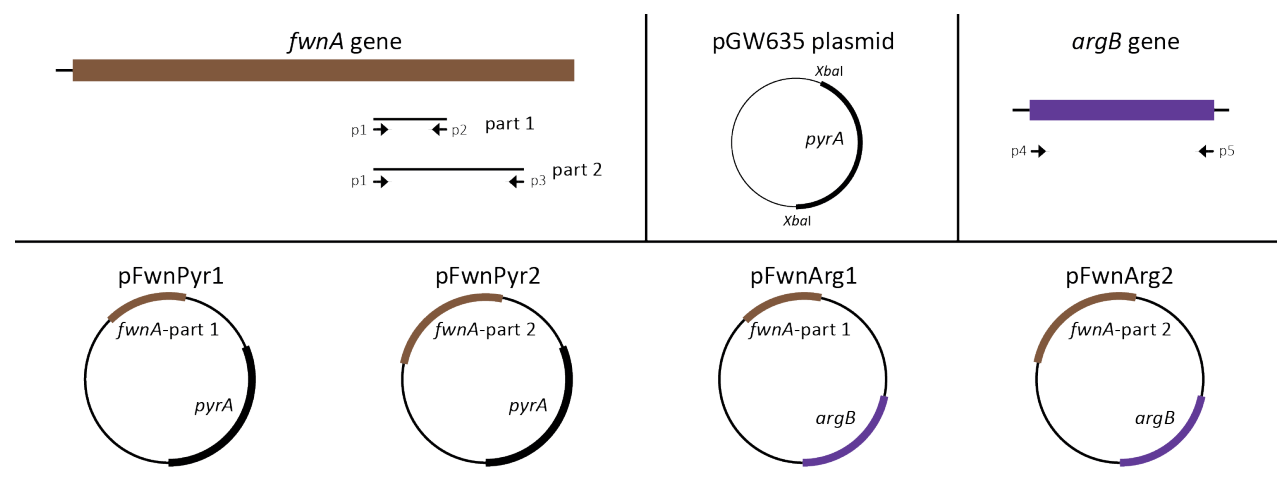

Figure 6.1 Cloning scheme for plasmids created in this study.

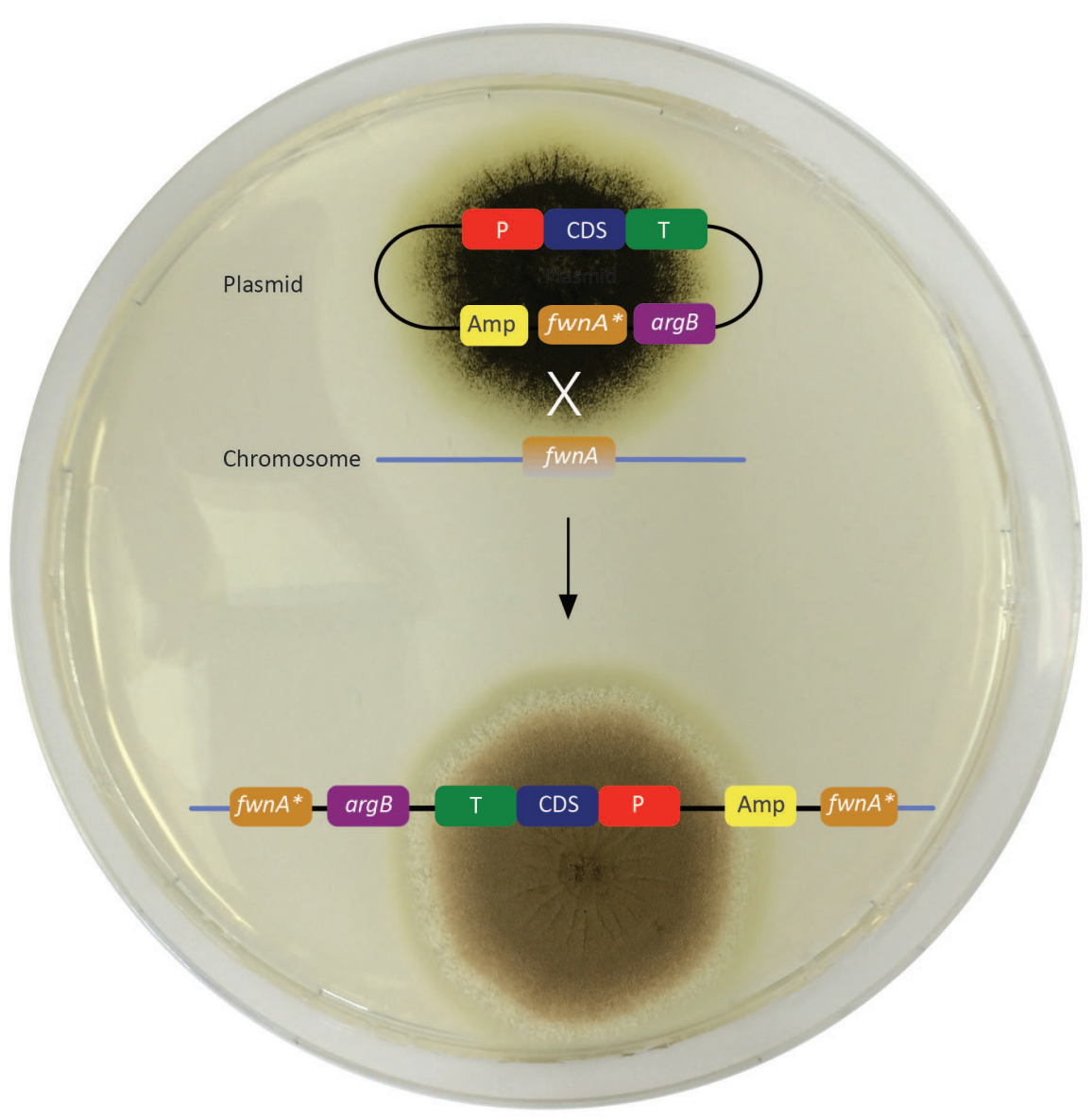

Figure 6.2 Schematic representation of the strategy used in this study. De black colored spores will turn fawn when the construct is integrated at the fwnA locus in the genome of Aspergillus niger. argB resembles the position of the fungal selection marker and could be replaced by pyrA. 
Transformations of strain N593 resulting from pFwnPyr1 did not result in the formation of fawn colored colonies on the primary transformation plates and purification plates. An explanation for this finding is that the truncated gene resulting from the integration event at the fwnA locus might still encode a partially active protein. Another explanation is that the fwnA fragment in pFwnPyr1 is too small to yield efficient homologous recombination, since homologous recombination in $A$. niger is known to require up to $2 \mathrm{~kb}$ of homologous flanks. ${ }^{11}$ To test the latter cause, and to determine whether an increase in size of the fwnA fragment leads to more effective homologous integration, the pFwnPyr2 plasmid was constructed. This plasmid contains a $2 \mathrm{~kb}$ fragment that was generated using the same forward primer but a different reverse primer (Fig. 1). Integration of this fragment at the fwnA locus is expected to result in a similar truncated protein as in the pFwnPyr1 case. In the case of pFwnPyr2, the fwnA fragment was approximately half the size of the pyrA sequence; a ratio of approximately 10-15\% fawn colonies were expected based on 30\% integration events being due to homologous integration. ${ }^{12} \mathrm{~A}$ total of 113 transformants were obtained, 5 of them having a FwnA phenotype. Additionally, 6 grey colonies were found. Purification of the grey colonies in standard MMS plates led to fawn colored colonies, indicating a possible osmolarity dependent phenotype. Osmolarity dependent phenotypes have been reported previously by Jørgensen et al. (2011), ${ }^{5}$ when spores of a specific transformant were found to be black on complete medium (CM) plates containing $1.2 \mathrm{M}$ sorbitol but fawn on standard $\mathrm{CM}$ plates. All the colonies that were initially black did not change color on the purification plates. Since the grey colonies led to fawn colonies after the purification step, 11 out of 113 transformants showed the FwnA phenotype, which corresponds with the expected $10 \%$.

For the transformation of N593 that was used in the two initial transformation experiments pyrA was used as a selection marker. In the strain the pyrA phenotype results from a point mutation, thus the gene is essentially still present in the genome and is a potential site for integration, leading to a lower efficiency of integration at the fwnA locus. To experimentally verify this, we used the NW186 strain that is an arginine auxotrophic strain resulting from a gene deletion. The same fwnA fragments were tested in combination with the $\arg B$ selection marker. Transformations of $\triangle \mathrm{argB}$ NW186 strain were performed with pFwnArg1 and pFwnArg2. In these plasmids there is no homology with the genome apart from the fwnA fragment. Therefore a higher ratio of fwnA transformants was expected.

No fwnA colonies were obtained from transformations with pFwnArg1. When using plasmid pFwnArg2, which carries a $2 \mathrm{~kb}$ fwnA fragment, 55 transformants were found of which 10 were fawn (Fig. 6.3) (2 grey colonies were found that became fawn after purification). 


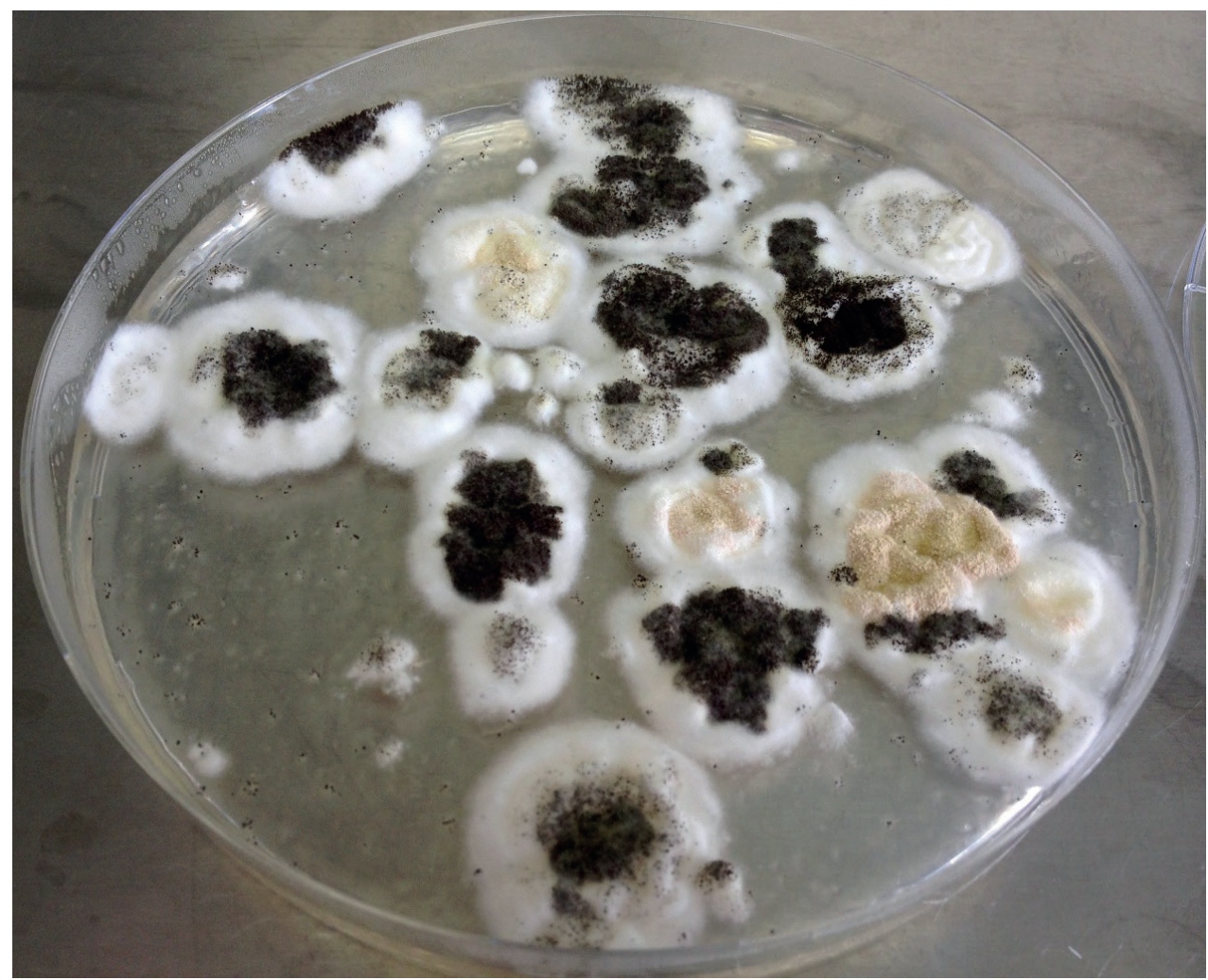

Figure 6.3 Example of a primary transformation plate after transformation of strain NW186 with pFwnArg2.

Also 2 white colonies were found; disruption of the fwnA locus was in some instances also correlated to white phenotypes. ${ }^{13}$ This supports our previous finding that the size of fragment 1 is indeed insufficient for homologous recombination at the fwnA locus. Another explanation is that a homologous recombination event of this fragment does not lead to loss-of-function of the gene product.

In this work the pyrA and $\arg B$ genes were specifically targeted to the fwnA locus of $A$. niger strains N593 and NW186 respectively, with a moderate success rate (approximately 20\% in the case of pFwnArg2). However, the event caused a direct change in the phenotype of the transformants, namely a change in spore color. This allows for easy screening for the desired transformants, which is a significant improvement over standard selection methods used for $A$. niger. Our results indicate that a fwnA fragment of at least $2 \mathrm{~kb}$ is required to provide sufficient homology for homologous recombination. As expected, the frequency of integration at the fwnA locus increases when homology of the vector with other regions of the fungal genome is removed. Based on the results of Jørgensen et al. (2011), ${ }^{5}$ we expected that integration of the constructs at the fwnA locus would only lead to fawn colonies. However, we found multiple phenotypes resulting from our experiments, 
mostly predominantly grey and white/colorless spores. Transferring grey colonies from the high osmolarity (1.2 M sucrose) stabilized MMS to standard MMS plates resulted in the appearance of only fawn colonies. This indicates an osmolarity dependent phenotype, with similar behavior as the colf mutant observed in the experiments previously described by Jørgensen et al. However, in that case transformants were black instead of grey in high osmolarity conditions. As mentioned, both these phenotypes have been linked to fwnA disruption, ${ }^{5,13}$ resulting in strains being unable to produce naphtho- $\gamma$-pyrones.

However, for our aim, a quick selection of homologous integration at a defined locus in $A$. niger, it is not important whether the fwnA- strains are colorless or fawn. Both phenotypes are easily distinguishable from wild-type strains and have been shown to be a reliable marker for integration at the fwnA locus. The method we have developed serves its purpose, though the frequency by which the desired transformants are found is low. This is due to the relatively low frequency of homologous integration in $A$. niger, ${ }^{11}$ but this can be drastically increased (>80\%) by the use of non-homologous end-joining-deficient (NHEJ) strains. ${ }^{14,15}$ Such strains can be generated by the disruption of $k u 70, k u 80$ or lig4. ${ }^{16}$

The use of this approach offers a non-laborious way of rapidly screening transformants that have the desired type of integration. Besides the fwnA gene, two other genes can be used that have been shown to affect spore color in knockout mutants, namely brnA (brown) and olvA (olive). ${ }^{5}$ The genes are epistatic over each other, with olvA being epistatic over brnA and fwnA being epistatic over both. This implies that integrations can be targeted to these genes sequentially when multiple transformations are required, to ensure easy screening of integration in the desired locus in every case.

\section{Competing interests}

The authors declare that they have no competing interests.

\section{Acknowledgements}

This work has been carried out on the basis of a grant in the framework of the BE BASIC program F01.002 Itaconic/fumaric acids: Novel Economic and eco-efficient processes for the production of itaconic and fumaric acid. 


\section{References}

1. Knuf C, Nielsen J. Aspergilli: Systems biology and industrial applications. Biotechnol J 2012,7:1147-1155.

2. Pel HJ, De Winde J, Archer DB, Dyer PS, Hofmann G, Schaap PJ et al. Genome sequencing and analysis of the versatile cell factory Aspergillus niger CBS 513.88. Nat Biotechnol, 2007,25:221231.

3. Lubertozzi D, Keasling JD. Developing Aspergillus as a host for heterologous expression. Biotechnol Adv 2009,27:53-75.

4. Carvalho NSP, Arentshorst M, Jin Kwon M, Meyer V, Ram AJ. Expanding the ku70 toolbox for filamentous fungi: establishment of complementation vectors and recipient strains for advanced gene analyses. Appl Microbiol Biotechnol 2010,87: 1463-1473.

5. Jørgensen TR, Park J, Arentshorst M, van Welzen AM, Lamers G, van Kuyk PA, et al. The molecular and genetic basis of conidial pigmentation in Aspergillus niger. Fungal Genet Biol 2011,48:544553.

6. Goosen T, Engelenburg F, Debet F, Swart K, Bos K, Broek H. Tryptophan auxotrophic mutants in Aspergillus niger: Inactivation of the trpC gene by cotransformation mutagenesis. Mol Gen Genet 1989,219:282-288.

7. Straat L van der, Tamayo-Ramos JA, Schonewille T, Graaff LH de. Overexpression of a modified 6-phosphofructo-1-kinase results in an increased itaconic acid productivity in Aspergillus niger. AMB Express, 2013,3:57

8. Kusters-van Someren MA, Harmsen JA, Kester HC, Visser J. Structure of the Aspergillus niger pelA gene and its expression in Aspergillus niger and Aspergillus nidulans. Curr Genet 1991,20:293-299.

9. Vishniac W, Santer M. The thiobacilli. Bacteriol Rev 1957,21:195-213.

10. Goosen T, Bloemheuvel G, Gysler C, de Bie DA, van den Broek HWJ, Swart AK. Transformation of Aspergillus niger using the homologous orotidine-5'-phosphate-decarboxylase gene. Curr Genet 1987,11:499-503.

11. Meyer V. Genetic engineering of filamentous fungi - Progress, obstacles and future trends. Biotechnol Adv 2008,26:177-185.

12. Graaff LH de, van den Broeck H, Visser J. Isolation and characterization of the Aspergillus niger puruvate kinase gene. Curr. Genet. 1992,1:21-27

13. Chiang Y-M, Meyer KM, Praseuth M, Baker SE, Bruno KS, Wang CCC. Characterization of a polyketide synthase in Aspergillus niger whose product is a precursor for both dihydroxynaphthalene (DHN) melanin and naphtho- - -pyrone. Fungal Genet Biol 2011,48:430437.

14. Meyer V, Arentshorst M, El-Ghezal A, Drews A-C, Kooistra R, van den Hondel CAMJJ, Ram AFJ. Highly efficient gene targeting in the Aspergillus niger kusA mutant. J Biotechnol, 2007,128:770775 . 
15. Kück $U$, Hoff B. New tools for the genetic manipulation of filamentous fungi. App/ Microbiol Biotechnol 2010,86:51-62.

16. Arentshorst M, Ram AJ, Meyer V. (2012) Using Non-homologous End-Joining-Deficient Strains for Functional Gene Analyses in Filamentous Fungi. Methods Mol Biol 2012,835:133-50. 



\section{6}

(9)
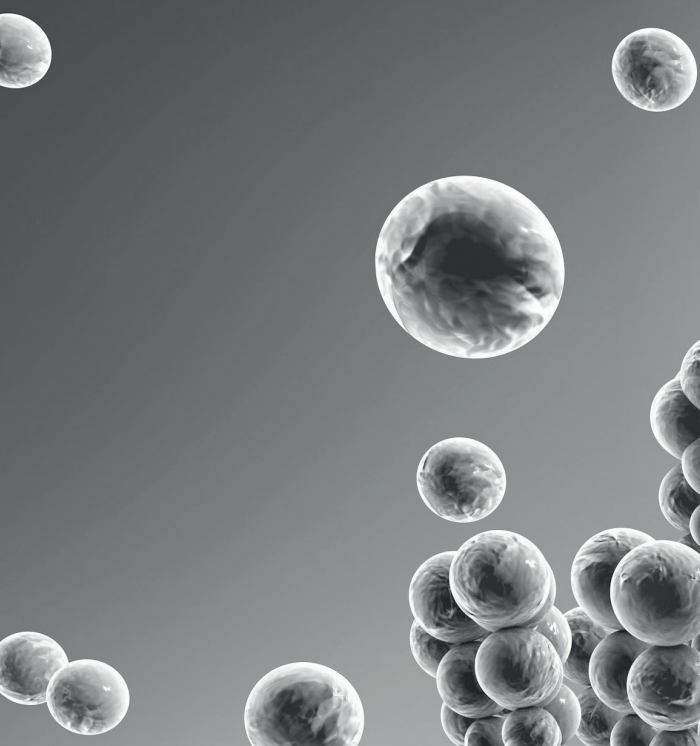

6

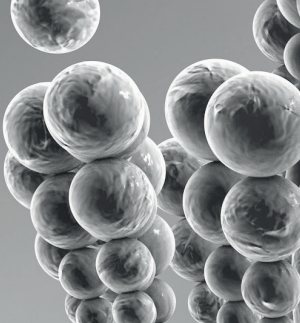

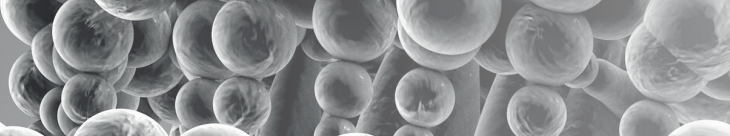
(1)
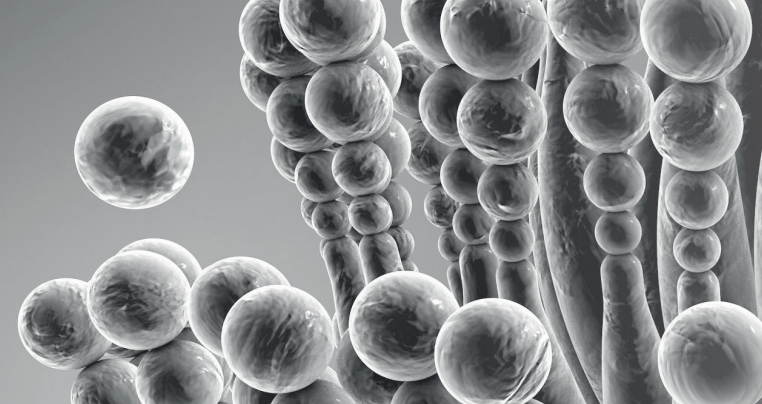

(9)
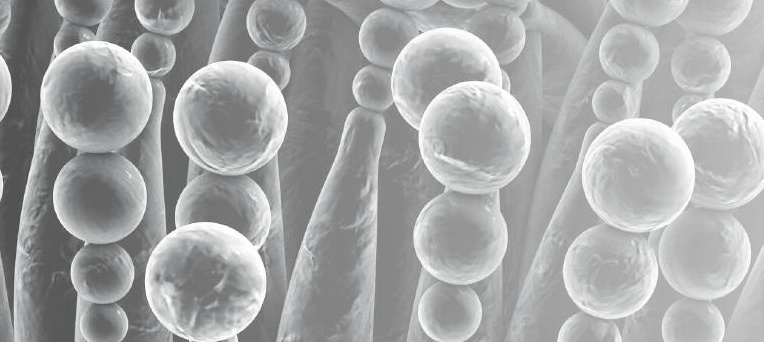

$$
\text { Q }
$$
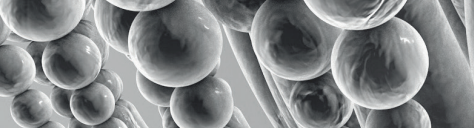

428

init 180

il mo

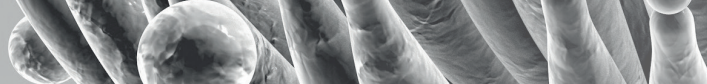

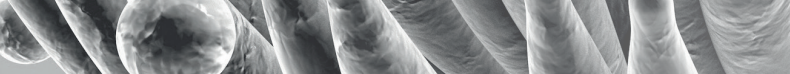

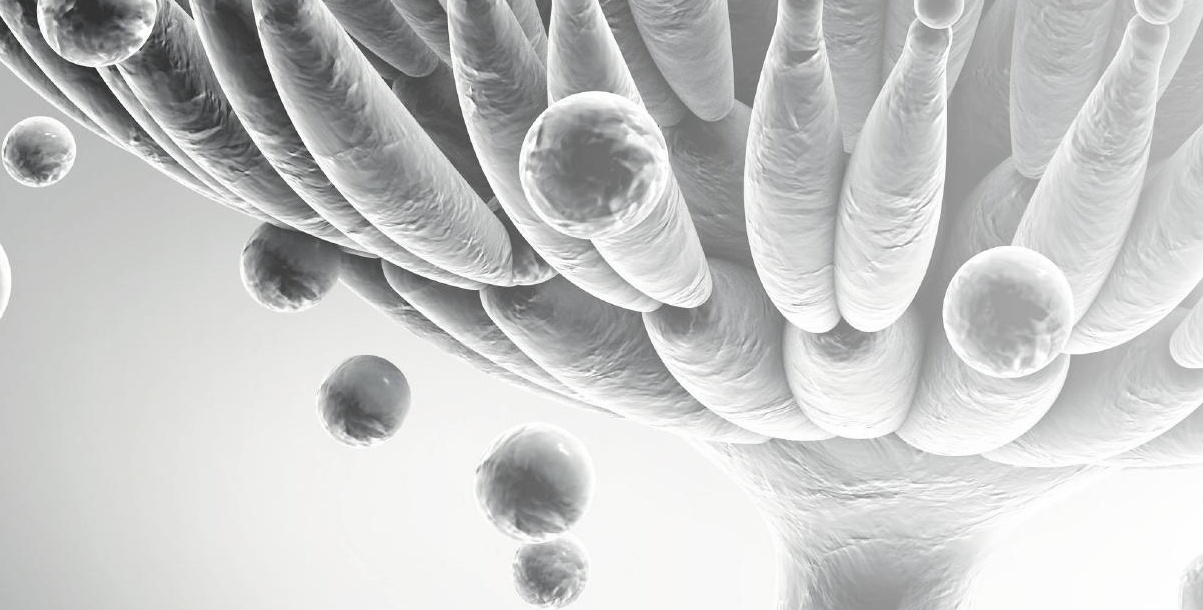

(.

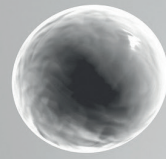

6 


\section{Chapter 7}

\section{Optimization strategies for microbial itaconic acid biosynthesis}




\section{Abstract}

\section{Background}

Itaconic acid is a C5 dicarboxylic acid that can serve as a building block to be used in industry to synthesize polymers that are currently based on petroleum-based components.

\section{Methods}

An overview of the recent literature on microbial itaconic acid production is given in this mini-review. The biosynthetic pathways as they are known in Aspergillus terreus and Ustilago maydis are described. Major advances have been made in the development of different microorganisms to serve as potential novel itaconic acid production hosts. Although fermentation strategies are discussed, our main focus is on metabolic engineering strategies for optimal itaconic acid biosynthesis.

\section{Results}

Itaconic acid is naturally produced by Aspergillus terreus, certain Ustilago and Candida species and Pseudozyma antarctica. Also in mammalian cells itaconic acid is found during macrophage activation. The biosynthetic pathway in $A$. terreus has been well studied and the crucial enzyme for itaconic acid synthesis was found to be cis-aconitate decarboxylase (CadA). On the one hand, optimization of itaconic acid production was done by optimizing fermentation processes and by applying metabolic engineering strategies to the natural producers, most of this was done with A. terreus. On the other hand, the identification of CadA allowed the exploration of heterologous expression of the cadA gene in different hosts. Since citric acid is the metabolic precursor for itaconic acid biosynthesis, many research efforts have focused on Aspergillus niger as a potential itaconic acid producer. The results of this research showed that besides the heterologous expression of cadA, transport between the different compartments and re-routing of the central carbon metabolism are important factors for the efficient biosynthesis of itaconic acid.

\section{Conclusion}

Several microorganisms have been investigated in the past years as potential itaconic acid producing hosts. Titers obtained by metabolic engineering of non-producing hosts range between $14.5 \mathrm{mg} / \mathrm{L}$ and $7.8 \mathrm{~g} / \mathrm{L}$. Although substantial progress has been made, the titers are not yet competitive with the titers obtained with the natural producer $A$. terreus. 


\section{Introduction}

The production of itaconic acid has gained increased interest ever since it was identified by the US department of Energy as one of the twelve building block chemicals that could potentially be produced from agricultural waste materials. Hence, this would implicate a biotechnological process in which this waste material is converted into high-value biobased chemicals. ${ }^{1}$ Itaconic acid or methylenesuccinic acid, is a C5 dicarboxylic acid that can be used in polymerization reactions to serve as a substitute for acrylic acid or methacrylic acid. ${ }^{2}$ As such itaconic acid is used in the production of synthetic resins, fibers, coatings, adhesives, plastics and rubbers. ${ }^{2,3,4}$

Here an overview is given of the organisms that are currently known to produce itaconic acid naturally and the fermentation strategies to optimize their yield. Also we review the metabolic engineering approaches that now have initiated the engineering of existing and novel hosts for the production of itaconic acid.

\section{Natural itaconic acid producing organisms}

Aspergillus terreus is the most well-known organism for itaconic acid production. The first report on itaconic acid production dates back to 1931 when Kinoshita et al. ${ }^{5}$ described itaconic acid production by Aspergillus itaconicus, a presumed $A$. terreus strain or a closely related species, which was isolated from the juice of salted plums. Besides $A$. terreus some Ustilago species ${ }^{6}$, Candida spec. ${ }^{7}$ and Pseudozyma antarctica ${ }^{8}$ are able to produce itaconic acid, in a concentration range from 30 to $53 \mathrm{~g} / \mathrm{L}$.

Recently, the synthesis of itaconic acid was also found in mammalian cells during macrophage activation. ${ }^{9}$ Itaconic acid plays a role as an antibiotic as part of a defense mechanism against pathogens like Mycobacterium tuberculosis and Salmonella enterica by inhibiting isocitrate lyase, the key enzyme of the glyoxylate shunt. The responsible gene in humans appears to be the immune-responsive gene 1 which encodes an enzyme having only $23 \%$ amino acid sequence identity to CadA from $A$. terreus, but that is catalyzing the same reaction. ${ }^{10}$

\section{Itaconic acid biosynthesis}

The biosynthetic pathway of itaconic acid in A. terreus has been studied since the beginning of the previous century. In contrast only just recently, in 2015, the itaconic acid biosynthesis pathway was identified in Ustilago maydis and was shown to be different from the pathway in A. terreus. 


\section{Biosynthetic pathway in Aspergillus terreus}

In 1931 Kinoshita et al. suggested that during synthesis of itaconic acid in A. itaconicus sugars, such as glucose and sucrose, are converted into gluconic acid, which is then further metabolized to citric acid that serves as a substrate for aconitic acid synthesis. ${ }^{5,11}$ The key reaction in the production of itaconic acid is the decarboxylation of aconitate. ${ }^{5} \mathrm{~A}$ few years later, Calam et al. published the results of their research that confirmed the synthesis of itaconic acid by $A$. terreus, but they could not support the theory that citric acid is a precursor for the production of itaconic acid. ${ }^{11}$ In 1957 three studies on the biosynthesis of itaconic acid in A. terreus in which they found evidence for the decarboxylation of cisaconitate by cis-aconitate decarboxylase (CadA) were published by Bentley and Thiessen. 12, 13, 14 It took another 55 years before the actual enzyme, cis-aconitate decarboxylase, that is responsible for the catalysis of the key reaction in itaconic acid biosynthesis, was isolated and characterized. ${ }^{15}$ In 2008, the encoding cadA gene was identified in A. terreus and expressed in Saccharomyces cerevisiae to confirm the activity. ${ }^{16}$ Around the same time, Li et al. ${ }^{17}$ used a clone-based transcriptomics approach to select and identify the cadA gene in $A$. terreus. In addition, they found that two genes that encode a putative mitochondrial transporter and a putative major facilitator superfamily transporter flank the cadA gene. Both these genes are highly expressed under itaconic acid producing conditions and are therefore possibly involved in the synthesis and secretion of itaconic acid. ${ }^{17}$ The three genes are also found to be linked to the lovastatin biosynthesis cluster as was found by Kennedy et al., 1999. ${ }^{18}$ The exact physiological correlation between lovastatin and itaconic acid biosynthesis remains unknown, but Lai et al. found a clear correlation between itaconic acid concentration and lovastatin production in A. terreus. When adding relatively low amounts of itaconic acid to the production medium, a significantly higher lovastatin production was observed.

Adding higher amounts of itaconic acid (>0.5 g/L) resulted in a negative effect on lovastatin production. Lovastatin production was also greatly reduced when itaconic acid was added during the germination phase instead of the production phase. ${ }^{19}$ This could possibly be the result of a $\mathrm{pH}$ effect, but the role of the lovastatin biosynthesis cluster being located next to the itaconic acid biosynthesis cluster and the co-regulation of these clusters remains intriguing. ${ }^{18}$

Jaklitsch et al. localized and found the CadA activity solely in the cytosol, the activity being absent in growing mycelia. ${ }^{20}$ Next to CadA, the activity and localization of nine other enzymes was studied in different cellular compartments during growth and itaconic acid production conditions. No significant differences in activity were found for citrate synthase, pyruvate dehydrogenase, 2-oxoglutarate dehydrogenase, $\mathrm{NAD}^{+}$: isocitrate dehydrogenase, $\mathrm{NADP}^{+}$: isocitrate dehydrogenase, $\mathrm{NAD}^{+}$: malate dehydrogenase, glucose6-phosphate dehydrogenase or pyruvate carboxylase. Of these, aconitase was the only enzyme that showed a significantly higher activity during itaconic acid production as 
compared to the growing, non-producing condition. The aconitase activity was mostly found in the mitochondrial fraction, suggesting that cis-aconitate is formed in the mitochondria and transported to the cytosol, where it is converted by means of CadA to itaconic acid. ${ }^{20}$

Itaconic acid biosynthesis was further studied in two A. terreus strains, a high-yield and low-yield strain, by using techniques that do not interfere with metabolism based on ${ }^{14} \mathrm{C}$ and ${ }^{13} \mathrm{C}$-labeled substrates in combination with NMR and mass spectroscopy. From the results it was concluded that the Embden-Meyerhof-Parnas pathway and Krebs cycle are involved in the formation of itaconic acid if glucose is used as a substrate (Fig. 7.1). ${ }^{21}$

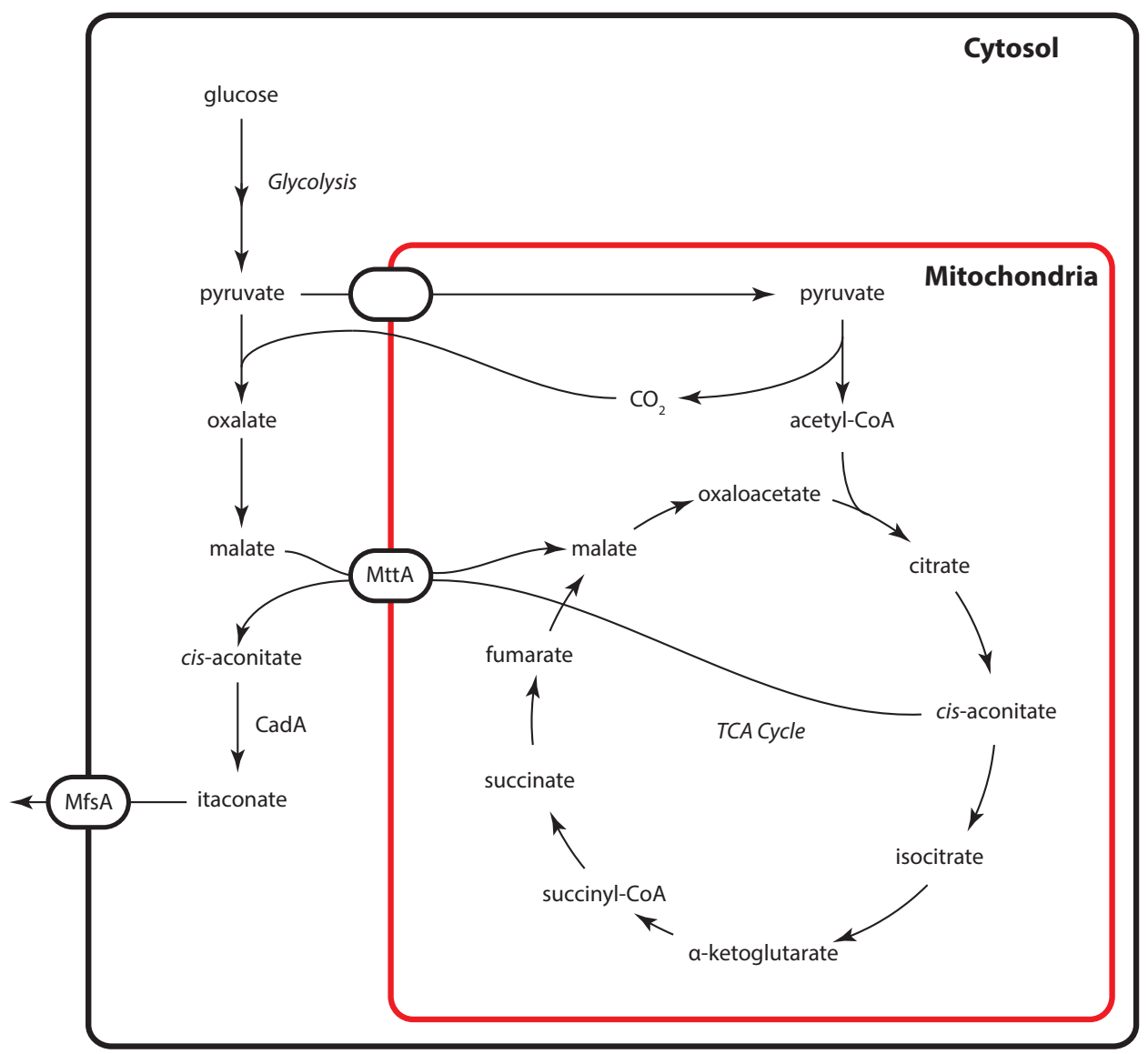

Figure 7.1 Schematic overview of biosynthesis pathway of itaconic acid in A. terreus. The cis-aconitate transport from the mitochondria to the cytosol by the transporter MttA was recently confirmed, the exchange for malate is still a supposition. ${ }^{22}$ Secretion of itaconic acid is assumed to occur by means of the putative transporter MfsA (adapted from Steiger et al $2013^{3}$ ). 


\section{Increasing itaconic acid production in natural producers by fermentation optimization}

Most of the studies on the optimization of itaconic acid production in natural producers involve strategies to increase itaconic acid production in $A$. terreus. There are only a few studies that cover the fermentation optimization of $U$. maydis or P. antarctica.

For the production of itaconic acid by $U$. maydis, a relatively low initial glucose concentration (50 g/L) appeared to be favorable over high initial glucose concentrations (100 or $200 \mathrm{~g} / \mathrm{L}$ ) in terms of yield $\left(\mathrm{Y}_{\mathrm{p} / \mathrm{s}}\right) .{ }^{24}$ Nitrogen limitation is necessary to achieve reasonable itaconic acid titers in both $U$. maydis, ${ }^{25}$ and P. antarctica. ${ }^{26}$ The optimal nitrogen concentrations that were found vary for the different studies. Whereas Klement and coworkers ${ }^{25}$ found that itaconic acid production was optimal in relatively low nitrogen concentrations, Maassen et al. ${ }^{24}$ found that relatively high nitrogen concentrations led to the highest itaconic acid titers. Maassen and coworkers suggested that the availability of oxygen differed in both studies and was the cause of the differences found.

Since $A$. terreus is the organism that is mostly used in industry for the production of itaconic acid, most of the research has focused on optimizing fermentation processes using this particular fungal strain.

Oxygen limitations during fermentation were found to result in an altered morphology, from filamentous mycelium into pellets, which dramatically decreased the production of itaconic acid. ${ }^{27}$

Itaconic acid production depends on the $\mathrm{pH}$ during fermentation. Several researchers investigated the effect of $\mathrm{pH}$ on itaconic acid production in $A$. terreus but different optima were found. The optimal initial $\mathrm{pH}$ ranges between 2.5 and 3.1, while the optimal pH during the production phase ranges between 2.1 and 2.8. A recent study showed an improvement in itaconic production by using $\mathrm{pH}$-controlled fermentation at $\mathrm{pH} 3$ during the production phase. ${ }^{28}$ An increased productivity of $1.15 \mathrm{~g} / \mathrm{L} / \mathrm{h}$, with a maximum productivity of 2.64 $\mathrm{g} / \mathrm{L} / \mathrm{h}$, was achieved by combining $\mathrm{pH}$ control, an optimized medium having increased $\mathrm{KH}_{2} \mathrm{PO}_{4}$ concentrations, and an increased fermentation temperature of $35^{\circ} \mathrm{C}$, resulting in a 2.8-fold and a 2.2-fold improvement, respectively. ${ }^{28}$

There is a growing interest in producing chemicals using renewable resources. In this context many research efforts have focused on itaconic acid production using renewable resources like corn starch, wheat flour, potato, cassava or sorghum. Examples of A. terreus strains producing itaconic acid from renewable resources were described by Petruccioli et al. and Dwiarti et al. ${ }^{29,30}$ 
Petruccioli et al. showed that A. terreus NRRL 1960 produced highest amounts of itaconic acid when corn starch was used as a substrate $(18.4 \mathrm{~g} / \mathrm{L})$, but the amount produced was highly dependent on the degree of hydrolysis of the starch. ${ }^{29}$ In another study using sago starch, Dwiarti et al. showed that $48.2 \mathrm{~g} / \mathrm{L}$ itaconic acid could be achieved using $A$. terreus TN484-M1. ${ }^{30}$ The usage of renewable resources for the production of itaconic acid has been extensively reviewed by El-Imam et al. ${ }^{31}$ In the following, we therefore focus on the metabolic engineering strategies that have been applied to microbial itaconic acid production.

\section{Metabolic engineering approaches to optimize itaconic acid production in Aspergillus terreus}

Several metabolic engineering strategies have been applied to improve itaconic acid production in A. terreus. One of these strategies involved the use of in vitro mutated cisaconitate decarboxylases. It appeared that $A$. terreus strains expressing CadA variants having one or more mutations in the C-terminal part of this enzyme showed increased enzymatic activity and produced higher levels of itaconic acid. ${ }^{32}$

Another approach to improve itaconic acid production by $A$. terreus is by increasing the flux towards the tricarboxylic acid (TCA) cycle, increasing the amount of substrate available leading to more product formation. One of these strategies was to increase the flux through glycolysis by modifying the allosteric properties of one of the glycolysis enzymes. The reaction catalyzed by 6-phosphofructo-1-kinase (PFK1) is a key reaction in the glycolysis pathway. It was found that, in contrast to the full-length enzyme, a shorter version of PFK1 is not inhibited by citrate or ATP. ${ }^{33}$

To make use of this feature, the pfkA gene was modified and tested in Aspergillus niger, which resulted in elevated citrate production. ${ }^{34}$ Expression of this modified $p f k A$ gene from A. niger in A. terreus resulted in enhanced itaconic acid levels as well. ${ }^{35}$

A second approach towards higher itaconic acid production in $A$. terreus involved the individual overexpression of each gene from the itaconic acid biosynthesis cluster; cadA, $m t t A$ and $m f s A .{ }^{36}$ This resulted in an increase of itaconic acid production of $9.4 \%$ for cadA, and $5.1 \%$ for $m f S A$, respectively. Overexpression of $m t t A$ did not increase itaconic acid production at all, most of the transformants showed slightly decreased production levels. Similarly, the overexpression of glyceraldehyde-3-phosphate dehydrogenase ( $g p d A)$, aconitase $(a c o A)$, citrate synthase (citA) and the mutated version of 6-phosphofructo1-kinase (mt-pfkA) did not increase itaconic acid production in this particular $A$. terreus strain. Nevertheless, overexpression of these genes did have an effect on the production 
of other acids, such as $\alpha$-ketoglutaric acid, malic acid, succinic acid, fumaric acid and cisaconitic acid. Thus, it might be that the key to success lies in the optimal combination of genes that are involved in the biosynthesis of itaconic aicd. The only combination tested in this research was $c a d A$ and $m f s A$, which showed better results than the overexpression of the individual genes in transformants. ${ }^{36}$

As the flux through the TCA cycle is oxygen dependent, continuous oxygen availability is an important prerequisite for itaconic acid production. Expression of the hemoglobin gene ( $\mathrm{vg} b$ ) from the Gram-negative bacteria Vitreoscilla in A. terreus M8 resulted indeed in elevated itaconic acid production. The heme group of the hemoglobin protein is able to scavenge oxygen from bulk water and thus enhances the intracellular oxygen availability. 37

In order to design a sustainable production process, $A$. terreus was modified to be able to efficiently metabolize corn starch. Overexpression of glucoseamylase in A. terreus under control of the native citrate synthase promoter showed improved itaconic acid production. Further improvements were made using the signal peptide of the major secreted protein ATEG_02176 (an acid phosphatase precursor) of A. terreus as a secretion signal for glucoseamylase combined with a two-step fermentation strategy wherein the first step comprises the germination of the spores using saccharified corn starch and the second step comprises the production phase using liquefied corn starch as substrate. This resulted in the production of $77.6 \mathrm{~g} / \mathrm{L}$ itaconic acid on corn starch which is very close to the industrial levels of $\geq 80 \mathrm{~g} / \mathrm{L}$ on glucose. ${ }^{38}$

\section{Metabolic engineering approaches to engineer novel hosts for itaconic acid production}

The genes encoding the metabolic enzymes involved in the production of itaconic acid have been heterologously expressed in several organisms to create novel itaconic acid production processes. Expression hosts included bacteria, yeasts and fungi.

\section{Itaconic acid production in Escherichia coli}

The first report on itaconic acid production in E. coli was the functional analysis of cadA from A. terreus by Li et al. A cadA gene codon-optimized for E. coli was expressed under control of an IPTG inducible promoter. The CadA protein was detected in SDS-PAGE and analysis of the culture medium using HPLC showed itaconic acid being produced, although in extremely low concentrations of less than $100 \mathrm{mg} / \mathrm{L}$. The transformants showed CadA activity after incubation with cis-aconitate. ${ }^{17}$ 
Later on, attempts were made to further optimize itaconic acid production using E. coli as production host. The expression of cadA resulted in inclusion bodies, which explains the low activity and thus itaconic acid concentrations. By reducing the cultivation temperature and making slight changes to the culture medium, the production could be improved to $240 \mathrm{mg} / \mathrm{L}$. Further improvements were achieved by introducing gltA and acnA from Corynebacterium glutamicum, encoding citrate synthase and aconitase respectively, on the one hand, and by deleting pta and IdhA, encoding phosphate acetyltransferase and lactate dehydrogenase, respectively, on the other hand. These deletions re-routed the carbon flow towards the production of pyruvate, which is a precursor for itaconic acid biosynthesis and led to an itaconate production of $690 \mathrm{mg} / \mathrm{L}$. ${ }^{39}$

\section{Itaconic acid production in Saccharomyces cerevisiae}

S. cerevisiae is often successfully used as a host for heterologous expression of genes. The cadA gene from $A$. terreus was heterologously expressed in S. cerevisiae to establish its CadA activity. ${ }^{16}$ More recently, a computationally guided approach was used to further optimize itaconic acid production in S. cerevisiae. An in silico knock-out analysis suggested several candidate genes that upon deletion result in an increased flux towards itaconic acid biosynthesis. Deletion of the genes ade3, bna2, both involved in amino acid synthesis and tes1 (peroxisomal acyl-CoA thioesterase) indeed resulted in increased itaconic acid levels although the genes were metabolically distant suggesting that itaconic acid synthesis is more complex than previously envisioned. The maximum itaconic acid titer that was achieved was still fairly low; $168 \mathrm{mg} / \mathrm{L} .{ }^{40}$

\section{Itaconic acid production in Corynebacterium glutamicum}

C. glutamicum is the host of choice for the production of L-glutamate and L-lysine. In the past, the organism was modified to produce a whole range of other industrially relevant metabolites such as L-serine, L-valine, L-isoleucine, L-leucine, D-lactate, succinate and pyruvate. Through expression of the for $C$. glutamicum codon-optimized cadA gene from $A$. terreus also itaconic acid production has been established in C. glutamicum. The best titers, $7.8 \mathrm{~g} / \mathrm{L}$ were obtained by reducing the isocitrate dehydrogenase activity. ${ }^{26}$

\section{Itaconic acid production in Yarrowia lipolytica}

Yarrowia lipolytica is a yeast that is known for its ability to grow on hydrophobic substrates and that is able to accumulate lipids. ${ }^{41}$ In addition, some $Y$. lipolytica mutants exist that are capable of producing high amounts of citric acid, up to $154 \mathrm{~g} / \mathrm{L}$. ${ }^{42}$ This makes these mutants interesting as a host for the production of itaconic acid, as it requires only the addition of one metabolic reaction step. Expression of cadA in Y. lipolytica resulted in fairly low itaconic acid levels, $33 \mathrm{mg} / \mathrm{L}$, but this level was increased to $4.6 \mathrm{~g} / \mathrm{L}$ by medium optimization and the simultaneous overexpression of the cadA gene and a gene that encodes for cytosolic aconitase from Y. lipolytica. ${ }^{43}$ 


\section{Itaconic acid production in Candida lignohabitans}

The yeast Candida lignohabitans is interesting as a production host due to its ability to grow on lignocellulosic biomass. Transformants of $C$. lignohabitans expressing the cadA gene from $A$. terreus and growing on glucose were able to produce 2-4 g/L itaconic acid. Using enzymatically-digested wood chips that contain mainly glucose $(47 \mathrm{~g} / \mathrm{L})$ and xylose $(17 \mathrm{~g} / \mathrm{L})$ as a carbon source, resulted in a maximum yield of $2.5 \mathrm{~g} / \mathrm{L}$ itaconic acid. ${ }^{44}$

\section{Itaconic acid production in Synechocystis sp. PCC6803}

An interesting approach towards a sustainable itaconic acid production process is to use $\mathrm{CO}_{2}$ as a substrate. Chin et al. were able to express CadA in the cyanobacterium Synechocystis sp. PCC6803. This led to very low itaconic acid levels, $14.5 \mathrm{mg} / \mathrm{L}$ at a production rate of 0.9 $\mathrm{mg} / \mathrm{L} /$ day. Nevertheless, this is the first example using photosynthesis to drive itaconic acid biosynthesis (Fig. 7.2). ${ }^{45}$

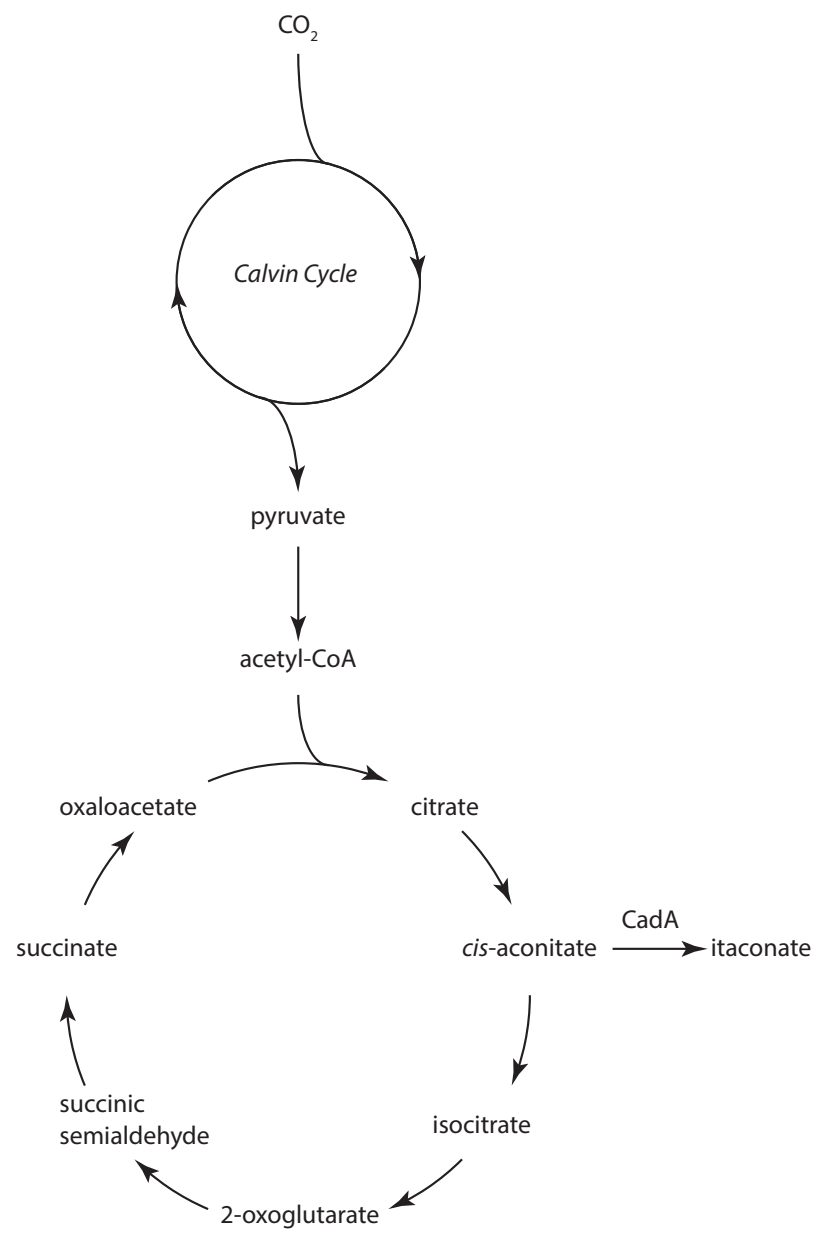

Figure 7.2 Itaconic acid production in Synechocystis sp. PCC6803, using $\mathrm{CO}_{2}$ as a substrate (adapted from Chin et al. ${ }^{45}$ ). 


\section{Metabolic engineering approaches for itaconic acid production in Aspergillus niger}

A. niger is an attractive host for itaconic acid production as its products are generally recognized as safe and the organism has a long history in the production of citric acid. As citric acid is the metabolic precursor in itaconic acid biosynthesis (Fig. 7.1), A. niger has a high potential to become the preferred host.

A. niger naturally does not produce itaconic acid since it lacks the essential gene encoding CadA. The obvious first step to prepare $A$. niger as a host for itaconic acid production is to express the cadA gene from $A$. terreus.

A first report describing the expression of cadA in $A$. niger AB1.13, a low citric acid producing strain, by $\mathrm{Li}$ et al., 2011, resulted in strains that produce $0.7 \mathrm{~g} / \mathrm{L}$ itaconic acid. ${ }^{17}$ Attempts to further improve the titers in this particular strain by overexpressing glyceraldehyde-3-phosphate dehydrogenase $(g p d A)$ did not raise itaconic acid levels although the transciptomics data showed a clear increase in transcript levels under itaconic acid producing conditions in A. terreus. ${ }^{17,45}$ Whereas overexpression of a flavohemoglobin domain ( $h b d 1)$ did influence the itaconic acid production in A. terreus in a positive way, ${ }^{37}$ in A. niger its overexpression did not yield higher titers. ${ }^{46}$

A study in A. niger using cadA for the application of constitutive promoters, showed a positive correlation between the promoter strength and the production of itaconic acid in A. niger. The strongest promoter in this study, $m b f A$, resulted in a maximum titer of 567 $\mathrm{mg} / \mathrm{L}$ itaconic acid. ${ }^{47}$

\section{Metabolite transport and compartmentalization}

Cellular compartmentalization requires intracellular transport of metabolites and specific localization of enzymes for optimal metabolic processes. Blumhoff et al. investigated the effect of enzyme compartmentalization by targeting CadA and aconitase to the cytosol or to the mitochondria. CadA naturally resides in the cytosol (cCadA). The mitochondrial localization was achieved by fusing a mitochondrial targeting signal to the cadA gene ( $m$ CadA). Three different aconitases were used in this study, two targeted to the cytosol and one targeted to the mitochondria. For the cytosolic aconitases the acnA (cAcnA) gene from $E$. coli and the aco1 (cAco1) gene from S. cerevisiae were expressed. The acoA (mAcoA) from $A$. niger was the mitochondria-targeted aconitase. Expression of the cCadA in combination with each of the three aconitase versions led to increased itaconic acid titers compared to the expression of cadA alone. Intriguingly, the combined expression of $c C a d A$ and the mitochondrial $m A c o A$ yielded a similar titer as the combined expression of $c C a d A$ and the cytosolic cAcnA. When both enzymes mCadA and $m A c o A$ were targeted to 
the mitochondria the itaconic acid titer was doubled to $0.8 \mathrm{~g} / \mathrm{L}$ on average. The highest titers were achieved by targeting both enzymes, cCadA, mCadA, cAcnA and mAcoA, to both compartments resulting in a final titer of $1.1 \mathrm{~g} / \mathrm{L}$ on average. ${ }^{48}$

As the cadA gene of $A$. terreus is flanked by two putative transporters, their potential role in metabolite transport in itaconic acid biosynthesis was investigated. The co-expression of these putative transporters with the cadA gene in $A$. niger $A B 1.13$ resulted in a slight increase in itaconic acid production to a final concentration of $1.5 \mathrm{~g} / \mathrm{L}$. The main acids produced by these transformants are still citric acid (up to $15 \mathrm{~g} / \mathrm{L}$ ) and oxalic acid (up to 4 g/L). ${ }^{49}$

\section{Re-routing carbon metabolism}

Besides citric acid, A. niger produces large amounts of oxalic acid and under certain conditions gluconic acid. Elimination of the biosynthesis of these organic acids would leave more carbon available for the biosynthesis of other metabolites and prevents the production of these side-products. This strategy was first employed by Ruijter et al. to increase citrate production. Although no effect was found on the citrate titers, an important result is the constitutive citrate biosynthesis in these strains. ${ }^{50}$ This important feature was the reason to choose $A$. niger NW186 as a platform organism to study itaconic acid production. This particular strain lacks oxalic acid production due to a mutation in the oahA gene encoding oxaloacetatehydrolase. This strain also does not produce gluconic acid as a result of a mutation in the gox $C$ gene that encodes glucose oxidase. ${ }^{51}$ Major improvements in itaconic acid titers were found by co-expression of the transporters MttA, MfsA and the cadA gene in A. niger NW186. The expression of the putative mitochondrial transporter (MttA) in combination with cadA resulted in a twenty-fold increase in itaconic acid production. Expression of both the mitochondrial transporter and the plasmamembrane transporter (MfsA) in combination with cadA resulted in a 25 -fold increase compared to the strain expressing only cadA. ${ }^{52}$ This confirmed the importance of metabolite transport between cellular compartments using an optimized host. ${ }^{53}$

\section{Elimination of allosteric regulation in glycolysis}

One of the strategies used for optimizing itaconic acid production in A. terreus was the elimination of the allosteric regulation in the glycolysis pathway by expressing a modified 6-phosphofructo-1-kinase ( $p f k A)$. The modified version of PfkA is no longer inhibited by citrate or ATP. ${ }^{33}$

Since the expression of the modified pfkA in A. niger leads to increased citrate synthesis, ${ }^{34}$ this could prove to be a valuable strategy to improve itaconic acid biosynthesis in $A$. niger as well. Combining the expression of the itaconic acid biosynthesis cluster, cadA and both transporters with the modified $p f k A$ gene did not result in higher production levels, but it did increase the itaconic acid productivity. ${ }^{54}$ 


\section{Pros and cons of different itaconic acid production hosts}

Several organisms have been investigated as a potential production host for itaconic acid biosynthesis. Important aspects for an efficient production process are the industrial experience with fermentation and metabolic engineering of the microorganism, a low $\mathrm{pH}$ tolerance of the host, the capability to use inexpensive substrates and finally the yield and productivity that can be achieved.

\section{Industrial experience}

Several of the microorganisms discussed here have a long history in industrial fermentations. Already since the 1950's itaconic acid is produced industrially in a fermentation process using A. terreus. ${ }^{2}$ A. niger is another host for which a lot of industrial experience has been developed over the years, since it is commonly used for the industrial production of organic acids like citric acid and enzymes. The commercial citric acid production started already in 1919. ${ }^{55}$

\section{Low pH tolerance}

In general, bacteria do not tolerate low pH just as well as e.g. fungi. With respect to this aspect, fungi as natural organic acid producers have a great advantage. The optimal $\mathrm{pH}$ for the itaconic acid biosynthesis in A. terreus is 2.8 , but even when the $\mathrm{pH}$ drops to 1.85 , itaconic acid is still being produced. ${ }^{56}$ The production process of citric acid is generally carried out using A. niger at a pH below 2 to avoid the loss of carbon via oxalic acid and gluconic acid biosynthesis. Oxalic acid synthesis and gluconic acid synthesis are inhibited at such low pH. ${ }^{50}$ Although E. coli is able to survive at a low $\mathrm{pH}$, production titers of organic acids decrease drastically when $\mathrm{pH}$ levels are not kept constant at neutral $\mathrm{pH}$.

An example is lactic acid synthesis in E. coli where titers vary between 50 and $75 \mathrm{~g} / \mathrm{L}$ with a controlled $\mathrm{pH}(\mathrm{pH}=7)$ while titers drop till $10-20 \mathrm{~g} / \mathrm{L}$ when $\mathrm{pH}$ is not controlled. ${ }^{57}$ Cyanobacteria like Synechocystis are known to be acid sensitive. A pH between 4.4 and 7.7 leads to acid stress responses, while a pH below 4.4 is lethal for Synechocystis. ${ }^{58}$ Production of salts of organic acids at neutral $\mathrm{pH}$ lead to large gypsum waste streams and need additional downstream processing steps to recover the acid form of the compound.

\section{Itaconic acid titers produced by different hosts}

As can be seen in Table 7.1, the itaconic acid titers obtained by different hosts vary considerably. The natural itaconic acid producing microorganisms $A$. terreus and $P$. antarctica are able to reach substantially higher itaconic acid titers as compared to the non-natural itaconic acid producing hosts. A. niger and C. glutamicum are able to synthesize itaconic acid at intermediate titers while the classical hosts for heterologous gene expression, such as $E$. coli and S. cerevisiae, are lagging behind in terms of titers. 
Table 7.1 Itaconic acid titers reached in fermentations of different microorganisms

\begin{tabular}{ll}
\hline Host & Titer \\
\hline A. terreus & $86 \mathrm{~g} / \mathrm{L}^{27}$ \\
P. antarctica & $30 \mathrm{~g} / \mathrm{L}^{8}$ \\
E. coli & $0.24 \mathrm{~g} / \mathrm{L}^{39}$ \\
S. cerevisiae & $168 \mathrm{mg} / \mathrm{L}^{40}$ \\
C. glutamicum & $7.8 \mathrm{~g} / \mathrm{L}^{26}$ \\
Y. lipolytica & $4.6 \mathrm{~g} / \mathrm{L}^{43}$ \\
C. lignohabitans & $2.5 \mathrm{~g} / \mathrm{L}^{44}$ \\
Synechocystis & $14.5 \mathrm{mg} / \mathrm{L}^{45}$ \\
A. niger & $7.1 \mathrm{~g} / \mathrm{L}^{52}$ \\
\hline
\end{tabular}

\section{Conclusion}

Over the past years, substantial progress has been made in microbial itaconic acid biosynthesis. The optimization of fermentation strategies and the metabolic engineering strategies that have been applied to the natural producer $A$. terreus have set the benchmark for itaconic acid production. Although itaconic acid production has been established in a variety of hosts so far, none of them reached titers that can compete with $A$. terreus. Studies on itaconic biosynthesis in $A$. niger have revealed the importance of metabolite transport between mitochondria, cytosol and the environment. Further optimization of transport within the different hosts might yield substantially higher titers. Besides transport, also rerouting of central carbon metabolism could further increase itaconic acid production as was shown in $A$. niger. In conclusion, the recent progress with itaconic acid production using non-natural hosts could potentially result in a variety of viable itaconic acid production platforms in the near future. 


\section{References}

1. Werpy T, Petersen G. Top Value Added Chemicals from Biomass: Volume I. Results of screening for potential candidates from sugars and synthesis gas. Pacific Northwest National Laboratory, National Renewable Energy Laboratory; 2004:1-76.

2. Willke T, Vorlop K-D. Biotechnological production of itaconic acid. Appl Microbiol Biotechnol 2001,56:289-295.

3. Steiger MG, Blumhoff ML, Mattanovich D, Sauer M. Biochemistry of microbial itaconic acid production. Front Microbiol 2013,4:1-5.

4. Okabe M, Lies D, Kanamasa S, Park EY. Biotechnological production of itaconic acid and its biosynthesis in Aspergillus terreus. App/ Microbiol Biotechnol 2009,84:597-606.

5. Kinoshita K. Über die Produktion von Itaconsäure und Mannit durch einen neuen Schimmelpilz, Aspergillus itaconicus. Acta Phytochim 1932,45:1-17.

6. Guevarra ED, Tabuchi T. Accumulation of Itaconic, 2-Hydroxyparaconic, Itatartaric, and Malic Acids by Strains of the Genus Ustilago. Agric Biol Chem 1990,54:2353-8.

7. Tabuchi T, Sugisawa T, Ishidori T, Nakahara T, Sugiyama J. Itaconic Acid Fermentation by a Yeast Belonging to the Genus Candida. Agric Biol Chem 1981,45:475-9.

8. Levinson WE, Kurtzman CP, Kuo TM. Production of itaconic acid by Pseudozyma antarctica NRRL Y-7808 under nitrogen-limited growth conditions. Enzyme Microb Technol 2006,39:824-7.

9. Strelko CL, Lu W, Dufort FJ, Seyfried TN, Chiles TC, Rabinowitz JD, et al. Itaconic Acid Is a Mammalian Metabolite Induced during Macrophage Activation. J Am Chem Soc. 2011, 133:16386-9.

10. Michelucci A, Cordes T, Ghelfi J, Pailot A, Reiling N, Goldmann O, Binz T, et al. Immune-responsive gene 1 protein links metabolism to immunity by catalyzing itaconic acid production. Proc. Nat/. Acad. Sci. 2013,110:7820-7825.

11. Calam CT, Oxford AE, Raistrick H. Studies in the biochemistry of micro-organisms: Itaconic acid, a metabolic product of a strain of Aspergillus terreus Thom. Biochem J 1939,33:1488-95.

12. Bentley R, Thiessen CP. Biosynthesis of itaconic acid in Aspergillus terreus. I. Tracer studies with C14-labeled substrates. J Biol Chem 1957,226:673-87.

13. Bentley R, Thiessen CP. Biosynthesis of itaconic acid in Aspergillus terreus. II. Early stages in glucose dissimilation and the role of citrate. J Biol Chem 1957,226:689-701.

14. Bentley R, Thiessen CP. Biosynthesis of itaconic acid in Aspergillus terreus. III. The properties and reaction mechanism of cis-aconitic acid decarboxylase. J Biol Chem 1957,226:703-20.

15. Dwiarti L, Yamane K, Yamatani H, Kahar P, Okabe M. Purification and characterization of cisaconitic acid decarboxylase from Aspergillus terreus TN484-M1. J Biosci Bioeng 2002,94:29-33.

16. Kanamasa S, Dwiarti L, Okabe M, Park EY. Cloning and functional characterization of the cisaconitic acid decarboxylase (CAD) gene from Aspergillus terreus. Appl Microbiol Biotechnol. 2008,80:223-9.

17. Li A, van Luijk N, Beek ter M, Caspers M, Punt P, van der Werf M. A clone-based transcriptomics approach for the identification of genes relevant for itaconic acid production in Aspergillus. Fungal Genet Biol 2011,48:602-11. 
18. Kennedy J, Auclair K, Kendrew SG, Park C, Vederas JC, Hutchinson CR. Modulation of polyketide synthase activity by accessory proteins during lovastatin biosynthesis. Science 1999,284:136872.

19. Lai L-ST, Hung C-S, Lo C-C. Effects of lactose and glucose on production of itaconic acid and lovastatin by Aspergillus terreus ATCC 20542. J Biosci Bioeng 2007,104:9-13.

20. Jaklitsch WM, Kubicek CP, Scrutton MC. The subcellular organization of itaconate biosynthesis in Aspergillus terreus. J Gen Microbiol 1991,137:533-9.

21. Bonnarme P, Gillet B, Sepulchre AM, Role C, Beloeil JC, Ducrocq C. Itaconate biosynthesis in Aspergillus terreus. J Bacteriol 1995,177:3573-8.

22. Steiger MG, Punt PJ, Ram AFJ, Mattanovich D, Sauer M. Characterizing MttA as a mitochondrial cis-aconitic acid transporter by metabolic engineering. Metab Eng 2016,35:95-104.

23. Geiser E, Przybilla SK, Friedrich A, Buckel W, Wierckx N, Blank LM, et al. Ustilago maydis produces itaconic acid via the unusual intermediate trans-aconitate. Microbial Biotech 2015,9:116-26.

24. Maassen N, Panakova M, Wierckx N, Geiser E, Zimmermann M, Bölker M, et al. Influence of carbon and nitrogen concentration on itaconic acid production by the smut fungus Ustilago maydis. Eng Life Sci 2014,14:129-34.

25. Klement T, Milker S, Jäger G, Grande PM, de María PD, Büchs J. Biomass pretreatment affects Ustilago maydis in producing itaconic acid. Microbial Cell Fact 2012,11:43.

26. Otten A, Brocker M, Bott M. Metabolic engineering of Corynebacterium glutamicum for the production of itaconate. Metab Eng 2015,30:156-65.

27. Kuenz A, Gallenmüller Y, Willke T, Vorlop K-D. Microbial production of itaconic acid: developing a stable platform for high product concentrations. Appl Microbiol Biotechnol 2012,96:1209-16.

28. Hevekerl A, Kuenz A, Vorlop K-D. Influence of the $\mathrm{pH}$ on the itaconic acid production with Aspergillus terreus. App/ Microbiol Biotechnol 2014,98:10005-12.

29. Petruccioli M, Pulci V, Federici F. Itaconic acid production by Aspergillus terreus on raw starchy materials. Lett Appl Microbiol 1999,28:309-12.

30. Dwiarti L, Otsuka M, Miura S, Yaguchi M, Okabe M. Itaconic acid production using sago starch hydrolysate by Aspergillus terreus TN484-M1. Bioresour Technol 2007,98:3329-37.

31. El-Imam AA, Du C. Fermentative itaconic acid production. Biodivers Biopros Dev 2014,1:1-8.

32. Hsieh HJ, Chang PC, Teng K. Cis-aconitate decarboxylase mutants having improved enzymatic activity. Patent US20100330631 A1, 2010.

33. Mesojednik S, Legisa M. Posttranslational modification of 6-phosphofructo-1-kinase in Aspergillus niger. Appl Environ Microbiol 2005,71:1425-32.

34. Capuder $M$, Šolar T, Benčina M, Legiša M. Highly active, citrate inhibition resistant form of Aspergillus niger 6-phosphofructo-1-kinase encoded by a modified pfkA gene. J Biotechnol 2009,144:51-7.

35. Tevž G, Benčina M, Legiša M. Enhancing itaconic acid production by Aspergillus terreus. App/ Microbiol Biotechnol 2010,87:1657-64.

36. Huang X, LU X, Li Y, Li X, Li J-J. Improving itaconic acid production through genetic engineering of an industrial Aspergillus terreus strain. Microb Cell Fact 2014,13:1-9. 
37. Lin $\mathrm{Y}-\mathrm{H}$, Li Y-F, Huang M-C. Intracellular expression of Vitreoscilla hemoglobin in Aspergillus terreus to alleviate the effect of a short break in aeration during culture. Biotechnol Lett 2004,26:1067-72.

38. Huang X, Chen M, LU X, Li Y, Li X, Li J-J. Direct production of itaconic acid from liquefied corn starch by genetically engineered Aspergillus terreus. Microb Cell Fact 2014,13:1-10.

39. Vuoristo KS, Mars AE, Sangra JV, Springer J, Eggink G, Sanders JPM, et al. Metabolic engineering of itaconate production in Escherichia coli. Appl Microbiol Biotechnol 2014,99:221-8.

40. Blazeck J, Miller J, Pan A, Gengler J, Holden C, Jamoussi M, et al. Metabolic engineering of Saccharomyces cerevisiae for itaconic acid production. Appl Microbiol Biotechnol 2014,98:815564.

41. Beopoulos A, Cescut J, Haddouche R, Uribelarrea JL, Molina-Jouve C, Nicaud JM. Yarrowia lipolytica as a model for bio-oil production. Prog in Lipid Res 2009,48:375-87

42. Rywińska A, Rymowicz W. High-yield production of citric acid by Yarrowia lipolytica on glycerol in repeated-batch bioreactors. J Ind Microbiol Biotechnol 2010,37:431-5.

43. Blazeck J, Hill A, Jamoussi M, Pan A, Miller J, Alper HS. Metabolic engineering of Yarrowia lipolytica for itaconic acid production. Metab Eng 2015,32:66-73.

44. Bellasio M, Mattanovich D, Sauer M, Marx H. Organic acids from lignocellulose: Candida lignohabitans as a new microbial cell factory. J Ind Microbiol Biotechnol 2015,42:681-91.

45. Chin T, Sano M, Takahashi T, Ohara H, Aso Y. Photosynthetic production of itaconic acid in Synechocystis sp. PCC6803. J Biotechnol 2015,195:43-5.

46. Li A, Pfelzer N, Zuijderwijk R, Punt P. Enhanced itaconic acid production in Aspergillus niger using genetic modification and medium optimization. BMC Biotechnol 2012,12:57.

47. Blumhoff M, Steiger MG, Marx H, Mattanovich D, Sauer M. Six novel constitutive promoters for metabolic engineering of Aspergillus niger. Appl Microbiol Biotechnol 2012,97:259-67.

48. Blumhoff ML, Steiger MG, Mattanovich D, Sauer M. Targeting enzymes to the right compartment: Metabolic engineering for itaconic acid production by Aspergillus niger. Metab Eng 2013,19:26-32.

49. Li A, Pfelzer N, Zuijderwijk R, Brickwedde A, van Zeijl C, Punt P. Reduced by-product formation and modified oxygen availability improve itaconic acid production in Aspergillus niger. Appl Microbiol Biotechnol 2013,97:3901-11.

50. Ruijter G, de Vondervoort van P. Oxalic acid production by Aspergillus niger: an oxalate-nonproducing mutant produces citric acid at pH 5 and in the presence of manganese. Microbiology 1999,145:2569-76.

51. Witteveen CFB, van de Vondervoort PJI, Swart K, Visser J. Glucose oxidase overproducing and negative mutants of Aspergillus niger. Appl Microbiol Biotechnol 1990,33:683-6.

52. van der Straat L, Vernooij M, Lammers M, van den Berg W, Schonewille T, Cordewener J, et al. Expression of the Aspergillus terreus itaconic acid biosynthesis cluster in Aspergillus niger. Microb Cell Fact. 2014,13:1-9.

53. van der Straat L, de Graaff LH. Pathway transfer in fungi. Bioengineered 2014,5:335-9.

54. van der Straat L, Tamayo-Ramos JA, Schonewille T, de Graaff LH. Overexpression of a modified 6-phosphofructo-1- kinase results in an increased itaconic acid productivity in Aspergillus niger. AMB Express 2013,3:57. 
55. Goldberg I, Rokem JS, Pines O. Organic acids: old metabolites, new themes. J Chem Technol Biotechnol 2006,81:1601-11.

56. Riscaldati E, Moresi M, Petruccioli M, Federici F. Effect of $\mathrm{pH}$ and stirring rate on itaconate production by Aspergillus terreus. J Biotechnol 2000,83:219-30.

57. Warnecke T, Gill RT. Organic acid toxicity, tolerance, and production in Escherichia coli biorefining applications. Microb Cell Fact 2005,4:25.

58. Huang JJ, Kolodny NH, Redfearn JT, Allen MM. The acid stress response of the cyanobacterium Synechocystis sp. strain PCC 6308. Archives of Microbiology 2002, 177:486-93. 



\section{6}

(9)
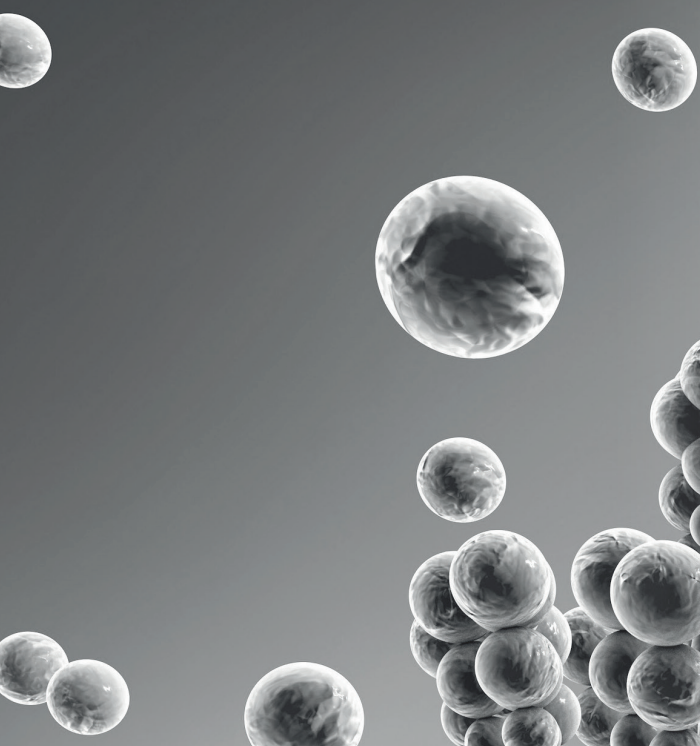

6

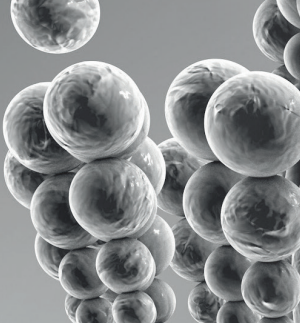

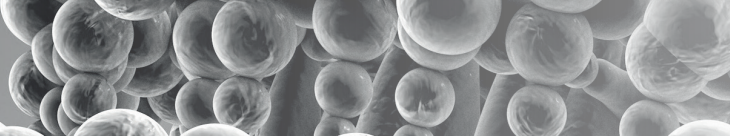
(1)
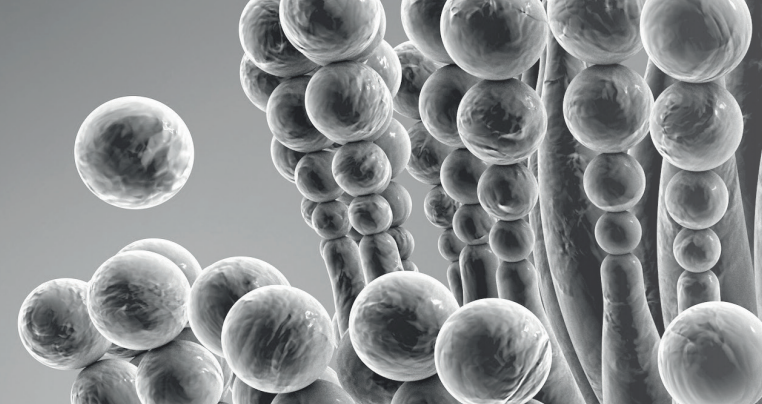

(9)
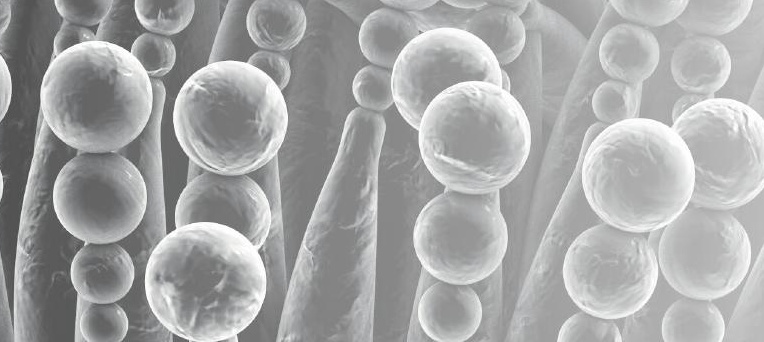

$$
\text { Q }
$$
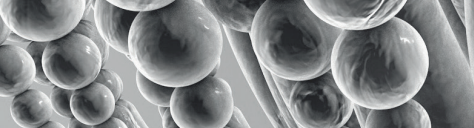

428

init 180

il mo

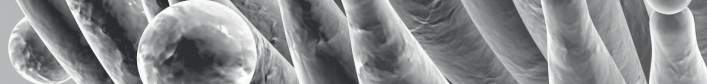

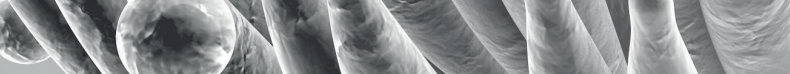

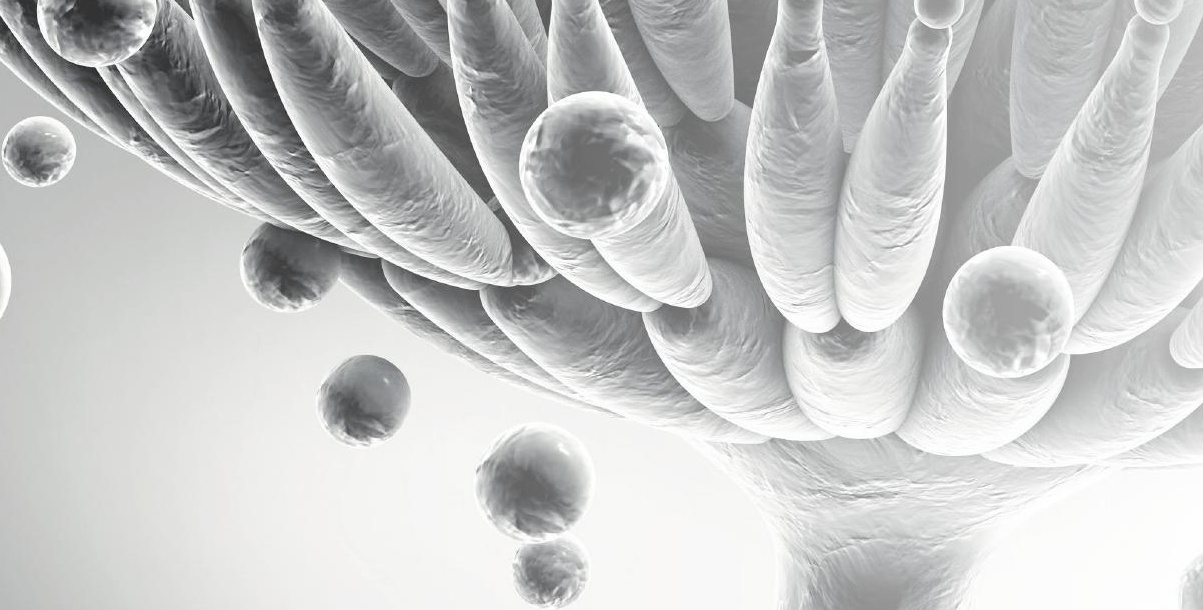

(.

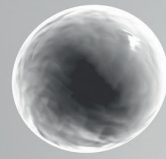

6 


\section{Chapter 8}

General Discussion 
In the past ten years the bio-based production of itaconic acid got an increased interest in the industrial and scientific field due to its ability to serve as a chemical building block and thereby avoiding the use of fossil resources. Next to the substitution of methacrylic acid and acrylic acid in polymerization reactions, itaconic acid can also be used to form compositions of polymers that cannot be produced with building blocks derived from petrochemical sources. Although itaconic acid is less reactive than methacrylic acid or acrylic acid, it can be applied for the production of coatings, drug capsules, shape memory polymers and elastomers. More applications might be developed in these particular fields using the promising crosslinking properties of itaconic acid-based polymers. ${ }^{1}$

Itaconic acid is naturally produced by several organisms like Aspergillus terreus, certain Ustilago and Candida species ${ }^{2}$ and Pseudozyma antarctica ${ }^{3}$, with the filamentous fungus Aspergillus terreus as the most well-studied. A more in depth overview of microbial itaconic acid biosynthesis has been given in Chapter $\mathbf{7}$.

Aspergillus niger is well-known for its industrial production of citric acid in fairly high titers. Since the metabolic step from citric acid to itaconic acid seemed quite small, the aim of this thesis was to investigate $A$. niger for its potential as a host in itaconic acid production. Three strategies were formulated to achieve this goal:

1. Introduction of the gene encoding the key enzyme cis-aconitate decarboxylase.

2. Studying the role of transporters that are potentially involved in itaconic acid production.

3. Optimization of the itaconic acid production by metabolic engineering.

During the course of this thesis it became clear that in order to introduce multiple genes in A. niger to establish and improve itaconic acid production, a more efficient transformation method would be helpful. Therefore, the Funbrick system (Chapter 5) and a method to visually select $A$. niger transformants (Chapter 6) were developed.

In this thesis, the A. niger strain NW186 (cspA1, goxC17, prtF28 $\triangle \arg B$, pyrA6) was chosen as host for the production of itaconic acid. This strain is a pyrA mutant of Aspergillus niger NW $185^{4}$ and has the advantage that it is a goxC17 and prtF28 mutant. The goxC17 mutation in glucose oxidase prohibits the formation of gluconic acid from glucose.

The prtF28 mutation inhibits the oxaloacetate hydrolase enzyme (OahA) that catalyzes the formation of oxalate from oxaloacetate. By preventing the formation of the by-products oxalate and gluconic acid a higher amount of substrate is available for conversion into itaconic acid and thus resulting in a more efficient production process. As will be explained in more detail below, the oxaloacetate produced by pyruvate carboxylase can also be reduced to malate to enter the mitochondrion in exchange for cis-aconitate, which can serve as a substrate again for itaconic acid production. 


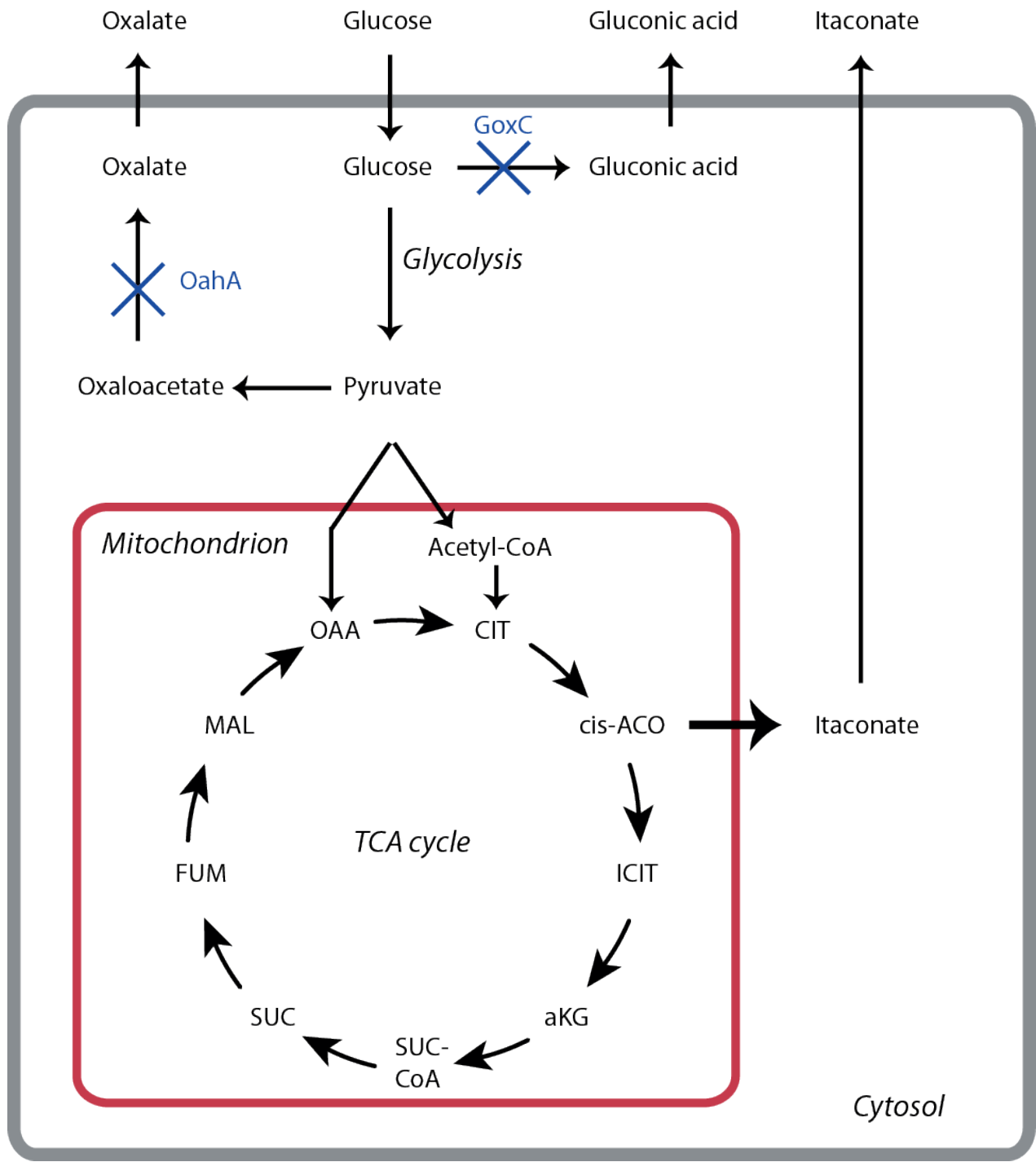

Figure 8.1 Metabolic pathway for the production of itaconic acid in A. niger NW186 (cspA1, goxC17, prtF28 $\triangle \arg B$, pyrA6). CIT = citrate, cis- $\mathrm{ACO}=$ cis-aconitate, $\mathrm{ICIT}=$ isocitrate, aKG = alpha ketoglutarate, SUC-COA = succinyl-Coa, SUC = succinate, FUM = fumarate, $\mathrm{MAL}=$ malate, $\mathrm{OAA}=$ oxaloacetate

Figure 8.1 provides a simplified metabolic pathway for the potential biosynthesis of itaconate from glucose in A. niger NW186. Blue crosses indicate both aforementioned mutations. The blockage of gluconic acid and oxalate formation in A. niger NW186 is advantageous to channel flux towards cis-aconitate because no carbon is lost via oxalate and gluconic acid formation. As such, A. niger NW186 is the preferred host for itaconic acid production. 


\section{Industrial feasibility}

Itaconic acid can serve as a building block for the bulk chemical industry. In order to obtain an industrially feasible process, high titers should be produced in a low-cost process. The titers obtained in the research described in this thesis are high from a scientific point of view but not yet high enough for an industrial process. In order to achieve titers and volumes that are competitive, the production process should be optimized and the process should be scaled up. In this thesis, an A. niger strain was used which is not used in industry. Itaconic acid production could potentially be greatly improved when an industrial citric acid production strain is used as a host and optimized production conditions are applied.

Another point of attention in creating an industrial feasible production process involves the choice of the substrate. Since itaconic acid is a bulk chemical and the retail price of bulk chemicals is low, the substrate should be very cheap. For scientific purposes, the experiments were done using sucrose as a substrate. During the course of this thesis we always kept in mind that this is not a substrate suited for an industrial process since the price is too high.

On the other hand, one should first establish and optimize the A. niger strain, the so called upstream process, before optimizing the downstream process in order to achieve the overall most efficient itaconic acid production process. One of the reasons that $A$. niger was chosen as host of interest is the fact that it has the ability to convert plant waste materials. In my opinion, the use of cheap materials like plant waste provides an interesting opportunity to further develop itaconic acid production towards an industrially feasible process.

Although we are aware of the importance of the industrial feasibility of itaconic acid production, the focus in this discussion was put on the more fundamental aspects of itaconic acid and citric acid production in A. niger.

\section{Introduction of cis-aconitate decarboxylase in A. niger}

The key enzyme for the biosynthesis of itaconic acid is cis-aconitate decarboxylase (CadA). The reaction in A. terreus whereby cis-aconitate is converted into itaconic acid (Fig. 8.2) was already discovered in the 1950s, but it took more than half a century to discover CadA and the cadA gene. ${ }^{5-7}$

Itaconic acid biosynthesis was established by introduction of the gene cadA in A. niger as shown in Chapter 2 but the obtained itaconic acid concentrations (0.05 g/L) were very low and far from the theoretical maximal production values $(135 \mathrm{~g} / \mathrm{L})$ based on citric acid production in $A$. niger. Especially for a compound that should serve as a building block in 


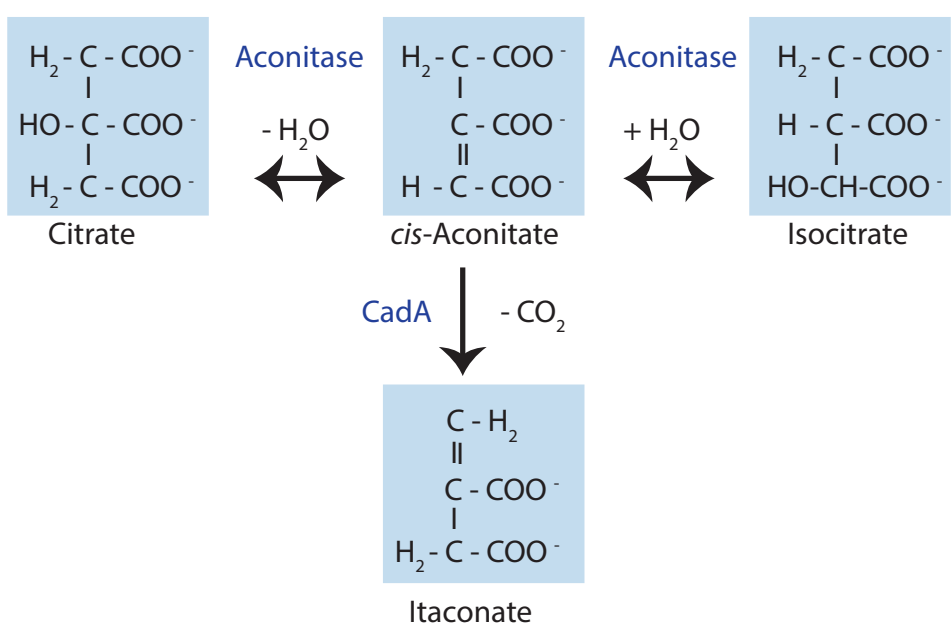

Figure 8.2 The key-reaction in A. terreus for the synthesis of itaconate from citrate via cis-aconitate catalysed by the enzymes aconitase and cis-aconitate decarboxylase (CadA). ${ }^{5,6}$

the bulk chemical industry, production levels should be higher. Similar low production levels were also shown by others and showed that expression of the key-enzyme on its own will not lead to a sustainable production process. ${ }^{8,9}$

\section{The role of transporters in itaconic acid production}

The filamentous fungus $A$. niger is a eukaryotic organism and as such, the glucose-based production of cis-aconitate is subject to compartmentalization (as shown in fig. 8.1). This important factor had to be taken into account when a new biosynthesis route for the production of itaconic acid was created. Citric acid and the precursor for itaconic acid biosynthesis cis-aconitic acid are products of the tricarboxylic acid cycle that is localized in the mitochondria (Fig. 8.1). So on the one hand, an internal transport process is required to transport metabolites from the mitochondria to the cytosol and on the other hand the export of itaconic acid from the cytosol is essential for an efficient production process.

Compartmentalization is a significant factor that should be kept in mind when introducing novel biosynthetic pathways in A. niger and filamentous fungi in general. Metabolite transport plays an important role in these organisms, but at the same time study of transporters, as described in Chapter $\mathbf{3}$, is complicated.

\section{MttA and MfsA transporters}

The cadA gene of $A$. terrreus was found to be flanked by two putative transporters, the putative mitochondrial transporter $m t t A$ and the putative plasma membrane transporter $m f s A$ (Fig. 8.3). ${ }^{10}$ Introduction of these transporters in A. niger transformants in which 
itaconic acid production was already established by expression of cadA resulted in major improvements in production levels as shown in Chapter $\mathbf{2}$.

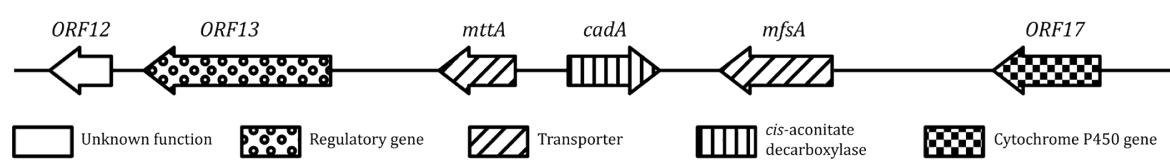

Figure 8.3 The itaconic acid biosynthesis cluster with cadA flanked by transporters $m t t A$ and $m f s A$ in the $A$. terreus genome (adapted from Kennedy et al.). ${ }^{10}$

The mitochondrial localization of the MttA transporter was confirmed by Steiger et al. (2016). ${ }^{11}$ It was also found that the preferred substrate for this particular transporter is cis-aconitate over citric acid and that it does not transport itaconic acid. This shows that the actual itaconic acid formation step takes place in the cytosol. Apparently, a bottleneck in the production of itaconic acid in A. niger strains expressing the key-enzyme CadA is the availability of the substrate cisaconitate in the correct compartment, in this case the cytosol.

The question that is still left is what the function of the MfsA transporter is. It is assumed that it is an itaconic acid exporter, but in Chapter $\mathbf{5}$ we did not observe a clear localization in the plasma membrane of $A$. niger. This might be due to mis-localization of the GFP construct. Hossain et al. pointed out that the sequence that was used in our study was slightly different compared to what was used in studies of Li et al. and Huang et al. ${ }^{12-14}$ This could also explain why different groups found different effects of overexpression of this particular transporter.

\section{Optimizing itaconic acid production by metabolic engineering}

Provided that transport is not a bottleneck, overexpression of genes involved in the biosynthesis of itaconic acid is a way to further improve production levels or productivity or both. Early research by Cleland and Johnson (1954) already showed that the biosynthesis of citric acid involves the formation of two moles of pyruvate via the glycolysis pathway. One mole of pyruvate is converted into acetyl-CoA, whereby one mole of carbon dioxide is released, while the other mole of pyruvate is converted into oxaloacetate, thereby incorporating one mole of carbon dioxide. ${ }^{15}$

The formation of acetyl-CoA occurs in the mitochondria while the carboxylation of pyruvate to oxaloacetate by pyruvate carboxylase takes place in the cytosol. ${ }^{16}$ Oxaloacetate is further reduced by malate dehydrogenase $(\mathrm{MDH})$ to malate (Fig. 8.4). Both acetyl-CoA and oxaloacetate are substrates for the formation of citrate, a reaction catalyzed by citrate synthase. This reaction occurs in the mitochondria. ${ }^{17}$ 


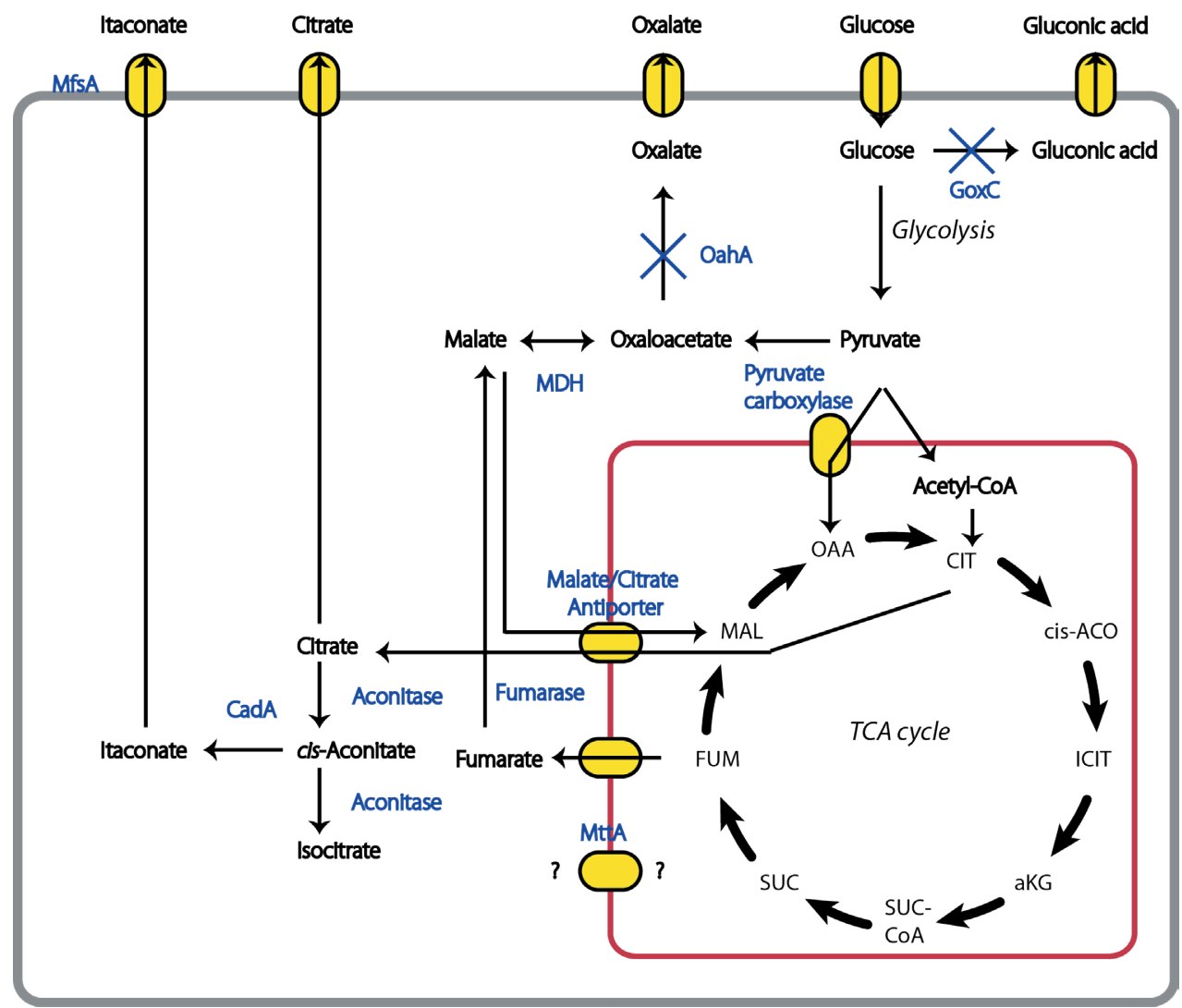

Figure 8.4 Metabolic pathway for the production of citric acid and itaconic acid in A. niger NW186 highlighting the metabolic reactions towards cytosolic malate. $\mathrm{CIT}=$ citrate, cis- $\mathrm{ACO}=$ cis-aconitate, $\mathrm{ICIT}=$ isocitrate, $\mathrm{aKG}=$ alpha ketoglutarate, $\mathrm{SUC}-\mathrm{CoA}=$ succinyl-COA, SUC = succinate, $\mathrm{FUM}=$ fumarate, $\mathrm{MAL}=$ malate, $\mathrm{OAA}=$ oxaloacetate.

\section{Overexpression citric acid biosynthesis pathway genes}

Attempts to increase citric acid production by overexpression of citA, encoding citrate synthase, in $A$. niger were not successful ${ }^{18}$, which suggested that this reaction is not a bottleneck for the production of citric acid. ${ }^{19}$ On the other hand, Hossain et al. (2016) recently showed that the overexpression of a putative cytosolic citrate synthase (citB) in $A$. niger expressing the itaconic acid biosynthesis cluster led to improved itaconic acid production. ${ }^{12}$ This highlights the importance of compartmentalization since CitA is a mitochondrial protein and CitB appears to be located in the cytosol.

Overexpression of the glycolytic enzymes phosphofructokinase and pyruvate kinase did not increase citrate levels either. ${ }^{20}$ Later, Capuder et al. (2009) showed that expression of a modified gene leads to a truncated phosphofructokinase whose activity was not inhibited by citrate. ${ }^{21}$ Overexpression of this particular gene in A. terreus showed increased levels of itaconic acid. ${ }^{22}$ 
In Chapter 4 of this thesis, we showed that overexpression of this modified 6-phosphofructo1-kinase in $A$. niger expressing the itaconic acid biosynthesis cluster enhances itaconic acid production. It did not lead to higher itaconic acid production levels, but the achieved production levels were obtained in a shorter time span, which is industrially interesting. Due to the removal of the citrate inhibition sites of the 6-phosphofructo-1-kinase enzyme, this step in the glycolysis pathway is no longer pinched upon high citrate concentrations.

\section{The role of cytosolic malate in acid production}

Although citric acid production in A. niger has been studied for over 100 years, it is still not exactly known how the fungus is able to produce such high levels of this metabolite. The idea that cytosolic malate is important in citric acid production is supported by the work of de Jongh and Nielsen. ${ }^{23}$ By overexpression of cytosolically targeted fumarases, Fum1s and FumRs from Saccharomyces cerevisiae and Rhizopus oryzae respectively, and malate dehydrogenase and fumarate reductase from $S$. cerevisiae, they were able to increase the yield and productivity of citric acid production in A. niger.

\section{Fumarase}

Fumarase or fumarate hydratase is an enzyme that catalyzes the reversible hydration of fumarate to malate. Fumarase is present in two forms, a mitochondrial and a cytosolic form (Fig. 8.4). The overexpression of fumarases from S. cerevisiae and R. oryzae in A. niger was successfully done by de Jongh and Nielsen (2008) and resulted in significantly increased citric acid productivities and yields. ${ }^{23}$ In order to see if we could obtain similar results for itaconic acid production in our particular $A$. niger strain, we overexpressed fumRs, the fumarase gene originating from $R$. oryzae, by co-transformation in our CAD4 strain, the A. niger NW186 strain expressing CadA from $A$. terreus. FumRs is a cytosolic fumarase and should therefore contribute to increased malate levels in the cytosol. Malate can be interchanged for cis-aconitate from the mitochondria, which in turn can be converted into itaconic acid. Interestingly, preliminary results of growth experiments in shake flasks showed increased citric acid levels (Fig 8.5) in nearly all transformants as compared to the host strain CAD4. Itaconic acid levels also increased slightly (Fig. 8.6) but they were still very low, which is as expected since we later found that the transporters are essential for efficient itaconic acid production. 


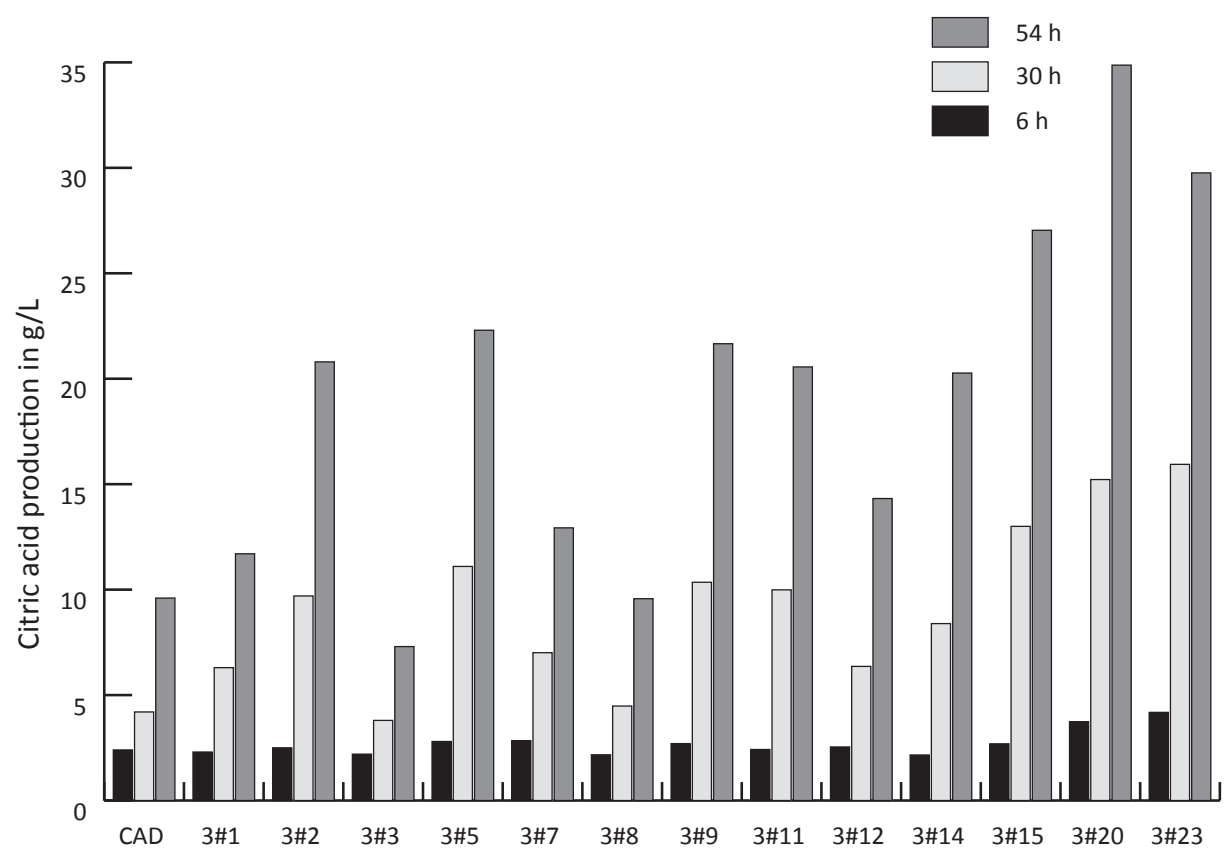

Figure $\mathbf{8 . 5}$ Citric acid production in transformants overexpressing FumRs. The host strain is CAD4, the A. niger NW186 strain expressing CadA from $A$. terreus.

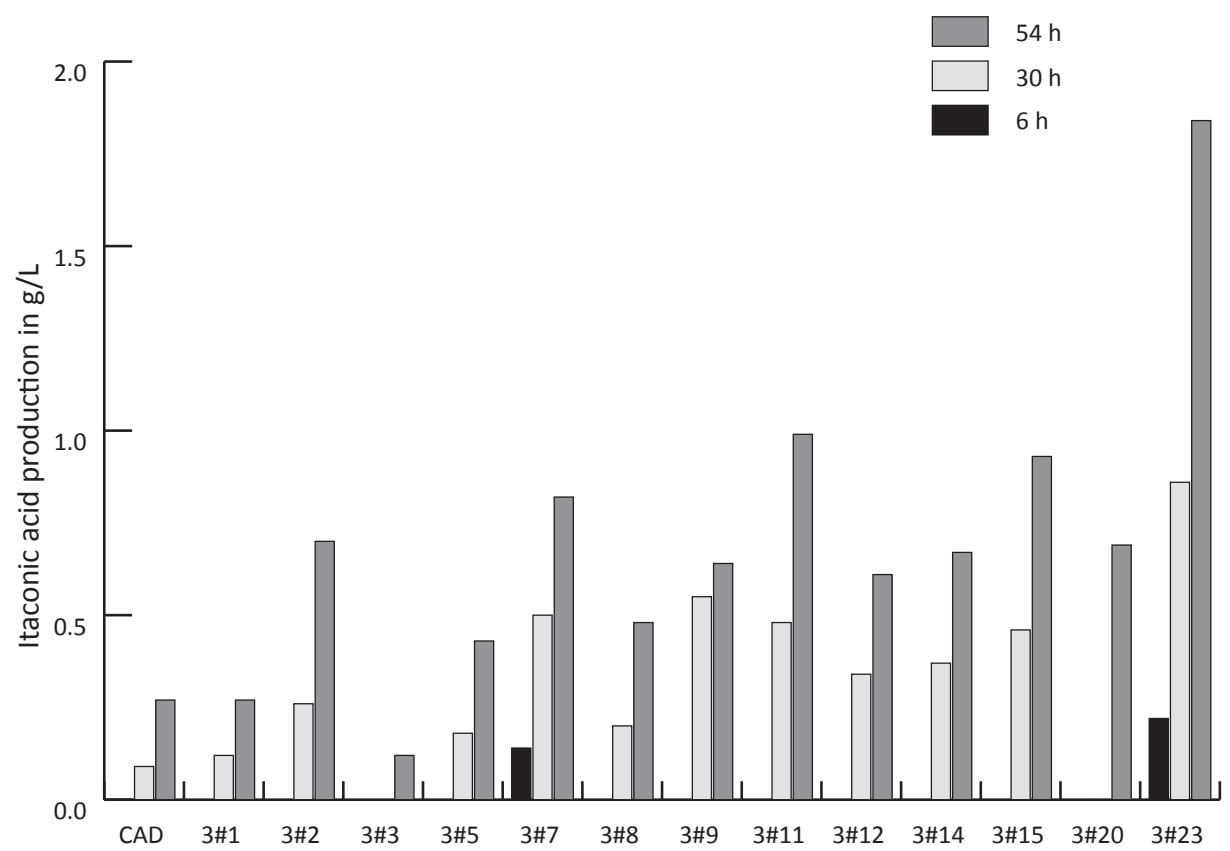

Figure $\mathbf{8 . 6}$ Itaconic acid production in transformants overexpressing FumRs. The host strain is CAD4, the A. niger NW186 strain expressing CadA from A. terreus. 


\section{Pyruvate carboxylase}

Pyruvate carboxylase indirectly also contributes to the formation of cytosolic malate by incorporating a carbon dioxide molecule in pyruvate to form oxaloacetate which can subsequently be used as a substrate for malate dehydrogenase for the formation of malate (Fig. 8.4). Pyruvate carboxylase (EC 6.4.1.1) of A. niger is a biotin-containing enzyme that uses ATP and $\mathrm{Mg}^{2+}$ as a cofactor. ${ }^{24}$ In several filamentous fungi, like Aspergillus nidulans ${ }^{25}$, Rhizopus oryzae ${ }^{26}$ and Aspergillus terreus ${ }^{17}$, pyruvate carboxylase activity is localized in the cytosol while in several $A$. niger strains it was found in both cytosolic and mitochondrial fractions. ${ }^{16}$ Interestingly, part of the $A$. niger strains showed higher specific activity of pyruvate carboxylase in the cytosol, while the other strains showed a higher activity in the mitochondria. So, the localization of pyruvate carboxylase enzyme activity is highly dependent on the particular A. niger strain. This appears not to be the case for other enzymes, such as citrate synthase and malate dehydrogenase, which showed a more similar distribution of enzyme activity throughout various compartments. ${ }^{16}$ It was suggested that for the production of several industrially relevant acids, like malic acid, citric acid, fumaric acid and itaconic acid, the cytosolic pathway via pyruvate carboxylase plays a major role as compared to the mitochondrial reactions in the TCA cycle. ${ }^{16,27}$

Therefore, we overexpressed cytosolic pyruvate carboxylase ${ }^{28}$ in A. niger NW186 to explore possible effects on citric acid production and in combination with the itaconic acid biosynthesis cluster to study the effects on itaconic acid production. The overexpression of fumarase and the work that was done on the transporters showed us that the transporters are crucial for itaconic acid production and therefore we decided to express pyruvate carboxylase only in combination with the complete itaconic acid cluster including the transporters.

The preliminary results showed that overexpression of pyruvate carboxylase in $A$. niger NW186 has a clear effect on citric acid production. Where normally titers of around $2 \mathrm{~g} / \mathrm{L}$ of citric acid are produced after 78 hours, some transformants reached almost $10 \mathrm{~g}$ citric acid per liter.

From the ten transformants that were tested in this growth experiment, which was performed in a similar way as the growth experiments described in chapter 4, nine produced clearly more citric acid that the parent strain. (Fig 8.7)

The overexpression of pyruvate carboxylase in A. niger NW186 in combination with the itaconic acid biosynthesis cluster did not show a clear effect (Fig 8.8). So in order to be able to draw a conclusion about the effect of overexpression of pyruvate carboxylase on itaconic acid production more research should be done. 


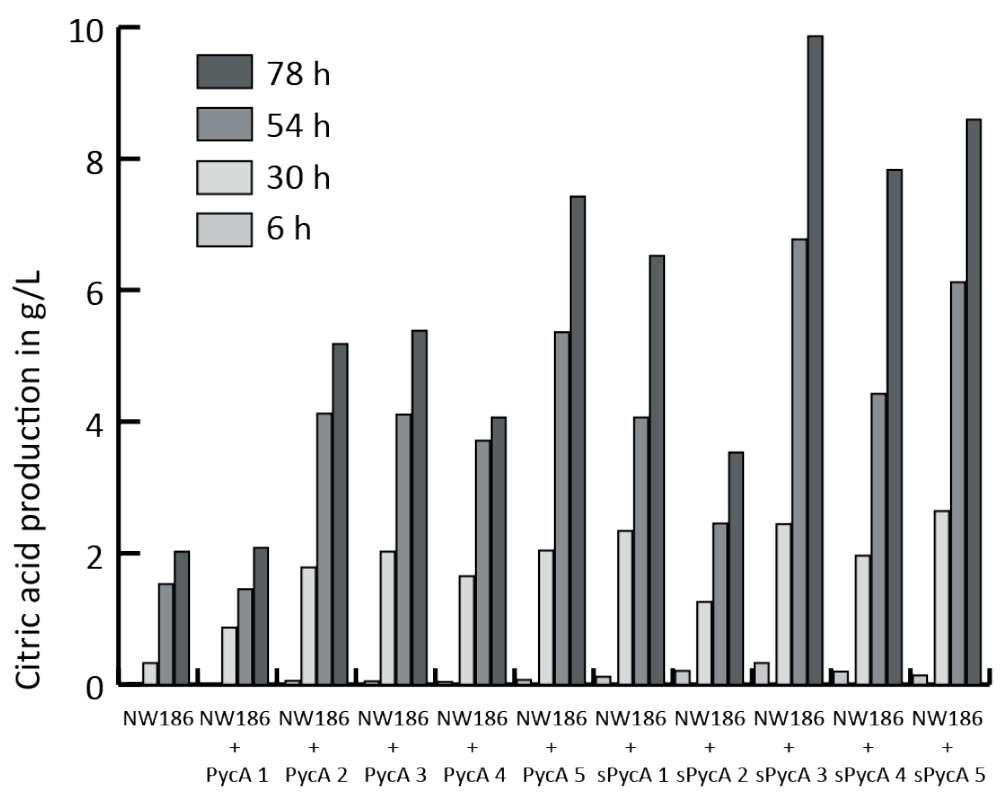

Figure 8.7 Citric acid production in $\mathrm{g} / \mathrm{L}$ in time by transformants overexpressing the endogenous pyruvate carboxylase gene (NW186 + PycA) or a synthetically made pyruvate carboxylase gene (NW186 + sPYCA) compared to the parent strain (NW186).

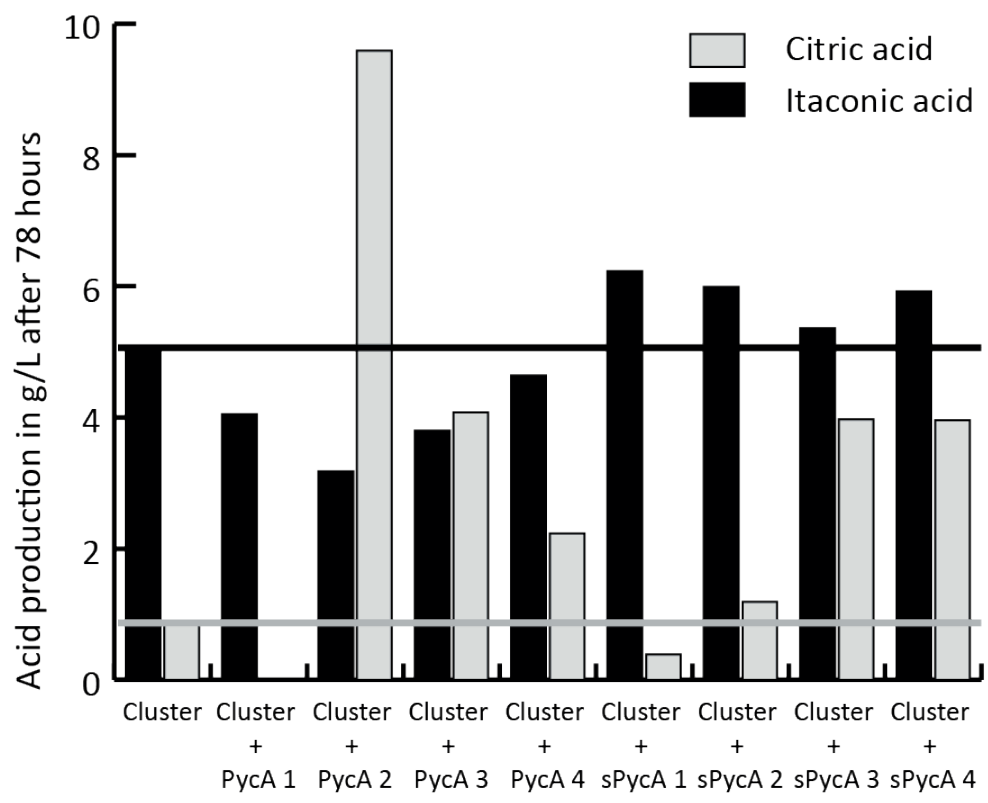

Figure $\mathbf{8 . 8}$ Citric acid and itaconic acid production in $\mathrm{g} / \mathrm{L}$ after 78 hours of growth in shake flasks by transformants overexpressing the endogenous pyruvate carboxylase gene (Cluster + PycA) or a synthetically made pyruvate carboxylase gene (Cluster + sPycA) in combination with the itaconic acid biosynthesis cluster compared to the highest itaconic acid yielding strain expressing cadA, $m t t A$ and $m f s A$ (Cluster). 


\section{Overexpression of a putative malate citrate antiporter}

We have seen that many strategies to improve citric acid production, and hence optionally itaconic acid production, in A. niger involve the increase of cytosolic malate. For yeast and rat liver mitochondria it was shown that a malate citrate antiporter plays a crucial role in the production of citrate. ${ }^{29,30}$ Mathematic modeling has predicted that such a malate citrate antiporter plays also a crucial role in the production of citric acid in A. niger. ${ }^{31}$ The general consensus is that citric acid produced in the mitochondria is exchanged for malate, which is produced in the cytosol (Fig. 8.4).

Thus far, it is still unknown if this antiporter exists and if it does, which gene encodes for it. In Pel et al. (2007) a prediction was made that gene An11g11230 encodes for this antiporter. ${ }^{28} \mathrm{~A}$ gene ontology annotation revealed that this particular gene encoding a transporter is probably involved in transmembrane transport (Uniprot, accessed March 2018). ${ }^{32}$

In order to study this An11g11230 (Mal/cit) gene, it was commercially synthesized. First, the localization of this transporter was confirmed to be mitochondrial by expressing the Mal/cit gene labeled with GFP (Fig 8.9).

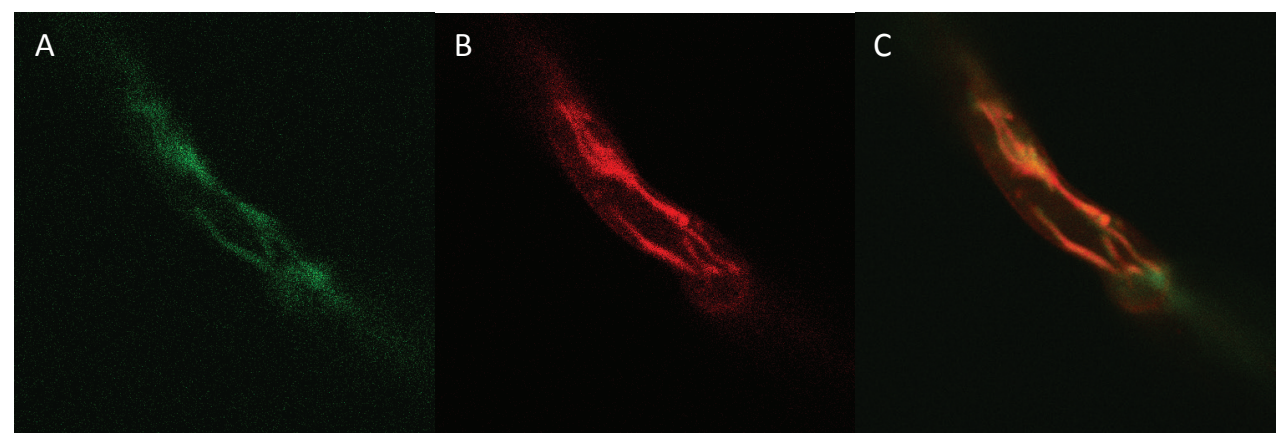

Figure 8.9 Localization of malate citrate antiporter labeled with GFP. A: GFP-Mal/cit, B: mitotracker, C: Merged picture. The putative malate citrate antiporter is clearly located in the mitochondria as visualized by using the mitotracker dye.

The effect of overexpressing the Mal/cit gene in A. niger NW186 on the citrate production was tested using 11 randomly chosen transformants in shake flask experiments in the same way as described in chapter 4. No clear effect was observed in these experiments. Several reasons can be the cause of this. Firstly, the gene An11g11230 does not encode the mitochondrial malate citrate antiporter. Secondly, in this particular strain and under these growth conditions, the malate citrate antiporter is not the bottleneck in citric acid production. Thirdly, the synthetic Mal/cit gene is not functional.

\section{Overexpression of a putative oxaloacetate transporter}

Although predicted to have a crucial function in citric acid production, the malate citrate antiporter of $A$. niger is still not discovered. Could it be that there is a different exchange process that is important in citric acid production and thus itaconic acid production? One interesting candidate for such an exchange was the mitochondrial oxaloacetate transporter 
that is responsible for the transport of oxaloacetate from the cytosol to the mitochondria. In this way, the mitochondria are supplied with substrate that can be converted into citrate that might be transported via another transporter to the cytosol.

In order to test this hypothesis, we overexpressed the putative oxaloacetate transporter gene (An14g06860), predicted by Pel et al. in 2007 28, in A. niger NW186. The obtained transformants were grown again in shake flask cultures to observe any effects on acid secretion. Interestingly, the citric acid levels produced by these transformants are slightly lower compared to the parent strain. The oxaloacetate transporter competes for cytosolic oxaloacetate with the malate dehydrogenase enzyme, potentially leading to a lower cytosolic malate concentration. Thereby, less malate is available to exchange for citrate via the putative malate citrate antiporter, which could explain the slightly reduced citrate production in these transformants.

\section{Methods developed for easy and efficient transformation of $A$. niger}

We started out by incorporating a single gene, cadA, into the A. niger NW186 genome, but this strategy quickly developed into the incorporation of multiple genes. As transformations in fungi can be cumbersome, an efficient transformation system for $A$. niger was required. The Biobrick system is a quite well known system for building expression vectors, but this system is mostly developed for expression in bacteria and in particular E. coli. Another disadvantage of the BioBrick system is that once constructed, components cannot be removed or substituted. This post-construction removal and substitution is a main feature enabled by the use of Funbricks (Chapter 5). The option to replace promoters, coding sequences, terminators and fungal selection markers appeared to be very helpful and efficient. Although the Funbrick system ensured a more efficient and less time-consuming way to make the constructs, they will probably not be used anymore in the near future since the option to order synthetically made constructs is becoming increasingly cheaper.

Another method that can be useful to speed up the time consuming process of fungal transformations and selection of transformants is described in Chapter 6 . The visual selection of targeted integration in $A$. niger by utilizing a change in spore color resulted in a quick and easy method to select transformants whose integration position is known to be in the locus of the gene responsible for the spore color formation.

\section{Future developments}

If we envision future improvements of biotechnological production processes in fungi, I think we can distinguish three different levels. First, we can look at the strain level and the way new strains are made using transformation events, selection of transformants and testing of 
the selected transformants on their performance. Second, we can look at the level of spatial arrangements and organization within the cell. ${ }^{33}$ Fungi are eukaryotic organisms that, contrary to prokaryotic organisms, have organelles and are compartmentalized. Transport of metabolites, enzymes, and other (macro) molecules between compartments and spatial localization of these molecules are impossible to ignore if optimal biological production processes are to be obtained. Transport processes within the cell are poorly understood, as transporters cannot be easily isolated and studied and overall little emphasis is given to studying transporters. ${ }^{34}$ As such, they are often overlooked and can easily become the bottleneck in the optimization of biological production processes. The third level at which biological production processes can be improved is the involved enzyme itself. All enzymes involved should work in an optimal manner and none of the enzymes should be inhibited in one way or another.

\section{Strain improvement}

Just a few decades ago, the idea of synthesizing a whole gene artificially was just a dream. Nowadays, it is possible to synthesize several kilobases of artificial DNA using little more than a thermocycler, oligonucleotides and DNA polymerase. ${ }^{35}$ Introducing these artificial genes in a host, is however, a different story. Many fungal hosts are not "genetically accessible" and when they are genetically accessible, transformation is often a time consuming step that in most cases requires manual labor as no universal method works for every fungal species. ${ }^{36}$ Also, the selection of transformants is time consuming and for many species difficult to automate. Although it might be difficult for some hosts, I do think that in the future many processes will be automated in order to obtain and screen thousands of transformants. ${ }^{37}$ This modern way of strain improvement is seen in industry as well.

Successful start-ups like Amyris and Zymergen are growing fast and are successfully exploring the world of automated strain improvement. ${ }^{38,39}$

\section{Spatial arrangements and organization within the cell}

If we take a closer look at a eukaryotic cell, I believe that besides the challenges there are also lots of opportunities to improve production of compounds by optimizing transport systems within the cell. Substrates and enzymes are not always physically in close proximity to each other. One could think of systems in which linkers and docking systems are used to place several enzymes of a production pathway next to each other to improve the production rate of the pathway as a whole. ${ }^{40-42}$

\section{Enzymes}

When we look at the enzymes themselves, there might be opportunities where enzymes can be modified in a similar way as was done with the modified 6-phosphofructo-1-kinase. If we know exactly which parts of the enzymes in the production pathway are end-product inhibition sites one could try to create a pathway that is not hampered by inhibition at all. 


\section{To conclude}

Itaconic acid production has been successfully established in A. niger, albeit in low concentrations, by expressing the gene encoding the key enzyme CadA. Expression of the transporters MttA and MfsA from A. terreus in A. niger showed that especially the mitochondrial MttA transporter has a major effect on the production of itaconic acid in the host strain A. niger NW186. This particular transporter has been characterized in the meantime and the hypothesized mitochondrial localization has been confirmed as well as the associated transport reaction of cis-aconitate from the mitochondria to the cytosol. ${ }^{11}$ The localization and functionality of the MfsA transporter is still unclear.

Overall, by focusing on transport processes, major improvements in itaconic acid production by $A$. niger have been achieved. 


\section{References}

1. Robert T, Friebel S. Itaconic acid - a versatile building block for renewable polyesters with enhanced functionality. Green Chem 2016,18:2922-2934

2. Steiger MG, Blumhoff ML, Mattanovich D, Sauer M. Biochemistry of microbial itaconic acid production. Front Microbiol 2013,4:23.

3. Levinson WE, Kurtzman CP, Kuo TM. Production of itaconic acid by Pseudozyma Antarctica NRRL Y-7808 under nitrogen-limited growth conditions. Enzyme Microb Technol 2006,39:824-827.

4. Ruijter GJ, de Vondervoort van PJ, Visser J. Oxalic acid production by Aspergillus niger: an oxalate-non-producing mutant produces citric acid at pH 5 and in the presence of manganese. Microbiology (Reading, Engl) 1999,145:2569-2576.

5. Bentley R, Thiessen CP. Biosynthesis of itaconic acid in Aspergillus terreus. III. The properties and reaction mechanism of cis-aconitic acid decarboxylase. J Biol Chem 1957,226:703-20.

6. Dwiarti L, Yamane K, Yamatani H, Kahar P, Okabe M. Purification and characterization of cisaconitic acid decarboxylase from Aspergillus terreus TN484-M1. J Biosci Bioeng 2002,94:29-33.

7. Kanamasa S, Dwiarti L, Okabe M, Park EY. Cloning and functional characterization of the cisaconitic acid decarboxylase (CAD) gene from Aspergillus terreus. Appl Microbiol Biotechnol 2008,80:223-229.

8. Li A, van Luijk N, Beek ter M, Caspers M, Punt P, van der Werf M. A clone-based transcriptomics approach for the identification of genes relevant for itaconic acid production in Aspergillus. Fungal Genet Biol 2011,48:602-11.

9. Blumhoff M, Steiger MG, Marx H, Mattanovich D, Sauer M. Six novel constitutive promoters for metabolic engineering of Aspergillus niger. Appl Microbiol Biotechnol 2012,97:259-67.

10. Kennedy J, Auclair K, Kendrew SG, Park C, Vederas JC, Hutchinson CR: Modulation of polyketide synthase activity by accessory proteins during lovastatin biosynthesis. Science 1999,284:13681372.

11. Steiger MG, Punt PJ, Ram AFJ, Mattanovich D, Sauer M. Characterizing MttA as a mitochondrial cis-aconitic acid transporter by metabolic engineering. Metab Eng 2016,35:95-104.

12. Hossain AB, Li A, Brickwedde A, Wilms L, Caspers M, Overkamp K, Punt PJ. Rewiring a secondary metabolite pathway towards itaconic acid production in Aspergillus niger. Microb Cell Fact 2016,15:130

13. Li A, Pfelzer N, Zuijderwijk R, Brickwedde A, van Zeijl C, Punt P: Reduced by-product formation and modified oxygen availability improve itaconic acid production in Aspergillus niger. Appl Microbiol Biotechnol 2013,9:3901-3911.

14. Huang X, Lu X, Li Y, Li X, Li J-J. Improving itaconic acid production through genetic engineering of an industrial Aspergillus terreus strain. Microb Cell Fact 2014,13:1-9.

15. Cleland WW, Johnson MJ. Tracer experiments on the mechanism of citric acid formation by Aspergillus niger. J Biol Chem 1954,208:679-690.

16. Bercovitz A, Peleg Y, Battat E, Rokem JS, Goldberg I. Localization of pyruvate carboxylase in organic acid-producing Aspergillus strains. Appl Environ Microbiol 1990,56:1594-1597. 
17. Jaklitsch WM, Kubicek CP, Scrutton MC. The subcellular organization of itaconate biosynthesis in Aspergillus terreus. J Gen Microbiol 1991,137:533-539.

18. Ruijter GJG, Panneman H, Xu D-B, Visser J. Properties of Aspergillus niger citrate synthase and effects of citA overexpression on citric acid production. FEMS Microbiol Lett 2000,184:35-40.

19. Ratledge C. Look before you clone. FEMS Microbiol Lett 2000,189:317-319.

20. Ruijter GJG, Panneman H, Visser J. Overexpression of phosphofructokinase and pyruvate kinase in citric acid-producing Aspergillus niger. Biochim Biophys Acta 1997,1334:317-326.

21. Capuder $M$, Šolar $T$, Benčina $M$, Legiša M. Highly active, citrate inhibition resistant form of Aspergillus niger 6-phosphofructo-1-kinase encoded by a modified pfkA gene. J Biotechnol 2009,144:51-57.

22. Tevž G, Benčina M, Legiša M. Enhancing itaconic acid production by Aspergillus terreus. Appl Microbiol Biotechnol 2010,87:1657-1664.

23. De Jongh WA, Nielsen J. Enhanced citrate production through gene insertion in Aspergillus niger. Metab Eng 2008,10:87-96.

24. Feir HA, Suzuki I. Pyruvate carboxylase of Aspergillus niger: kinetic study of a biotin-containing carboxylase. Can J Biochem 1969,47:697-710.

25. Osmani SA, Scrutton MC. The sub-cellular localisation of pyruvate carboxylase and of some other enzymes in Aspergillus nidulans. Eur J Biochem 1983,133:551-560.

26. Osmani SA, Scrutton MC. The sub-cellular localisation and regulatory properties of pyruvate carboxylase from Rhizopus arrhizus. Eur J Biochem 1985,147:119-128.

27. Kubicek CP. The role of the citric acid cycle in fungal organic acid fermentations. Biochem Soc Symp 1987,54:113-26.

28. Pel HJ, de Winde J, Archer DB, Dyer PS, Hofmann G, Schaap PJ et al.: Genome sequencing and analysis of the versatile cell factory Aspergillus niger CBS 513.88. Nature Biotechnol 2007,25:221-231.

29. Evans CT, Scragg AH, Ratledge CA. Comparative study of citrate efflux from mitochondria of oleaginous and non-oleaginous yeasts. Eur J Biochem 1983,130:195-204.

30. Bisaccia F, de Palma A, Dierks T, Krämer R, Palmieri F. Reaction mechanism of the reconstituted tricarboxylate carrier from rat liver mitochondria. Biochim Biophys Acta 1993,1142:139-145.

31. García J, Torres N. Mathematical modelling and assessment of the $\mathrm{pH}$ homeostasis mechanisms in Aspergillus niger while in citric acid producing conditions. J Theor Biol 2011,282:23-25.

32. Uniprot, Retrieved from www.uniprot.org accessed March 2018.

33. Harold FM. Molecules into Cells: Specifying Spatial Architecture. Microbiol Mol Biol Rev 2005,69:544-64.

34. Kell DB, Swainston N, Pir P, Oliver SG. Membrane transporter engineering in industrial biotechnology and whole cell biocatalysis. Trends Biotechnol 2015,33:237-46.

35. Hughes RA, Miklos AE, Ellington AD. Gene Synthesis: Methods and Applications. Methods Enzymol 2011,498: 277-309.

36. Li D, Tang Y, Lin J, Cai W. Methods for genetic transformation of filamentous fungi. Microb Cell Fact 2017,16:168. 
37. Lee SY, Kim HU. Systems strategies for developing industrial microbial strains. Nat Biotechnol 2015,22:1061-1072.

38. Amyris, retrieved from amyris.com, accessed May 2018.

39. Zymergen, retrieved from www.zymergen.com, accessed May 2018.

40. Bayer EA, Morag E, Lamed R. The cellulosome - A treasure-trove for biotechnology, Trends Biotechnol 1994,12:379-86.

41. Wang Y, Yu O. Synthetic scaffolds increased resveratrol biosynthesis in engineered yeast cells $J$ Biotechnol 2012,157:258-260.

42. Dueber JE, Wu GC, Malmirchegini GR, Moon TS, Petzold CJ, Ullal AV, Prather KLJ, Keasling JD. Synthetic protein scaffolds provide modular control over metabolic flux. 2009,27: 753-759. 



\section{6}

(9)
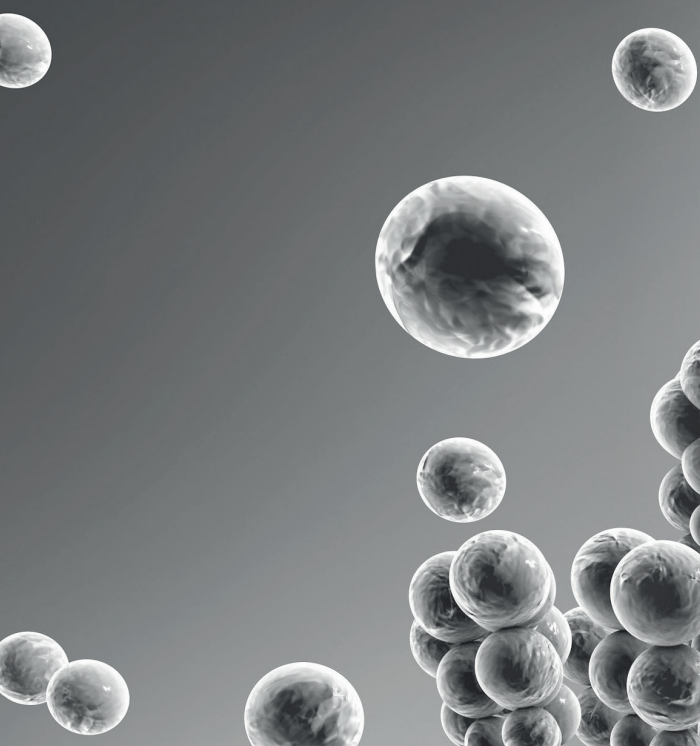

6

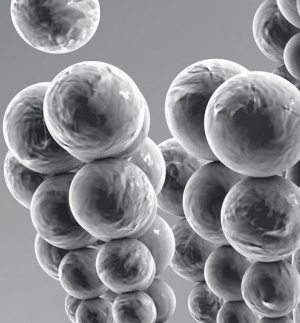

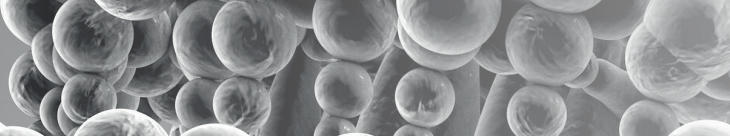
(1)
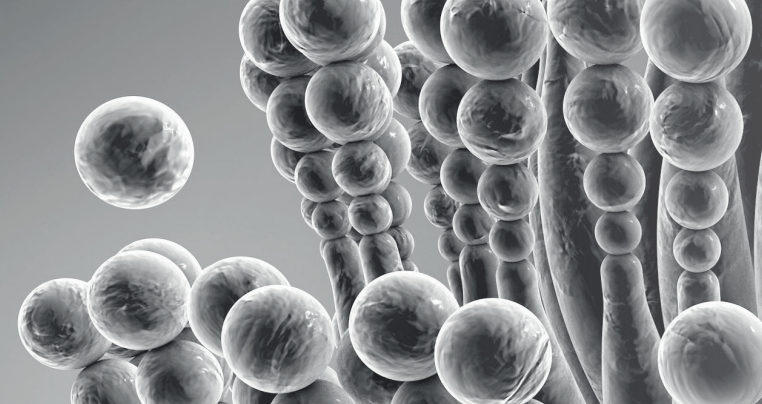

(9)
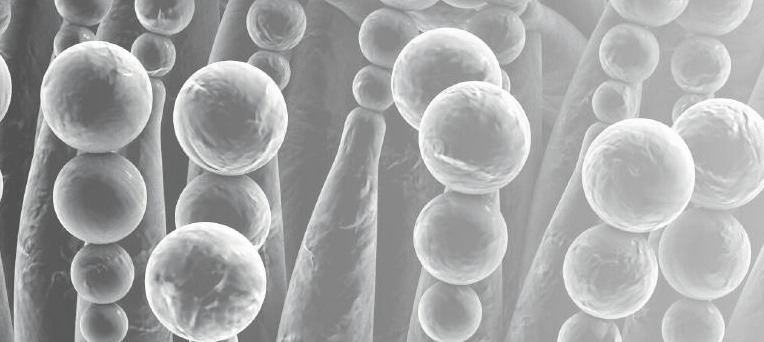

$$
\text { Q }
$$
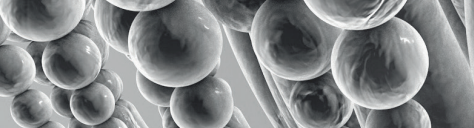

428

init 180

il mo

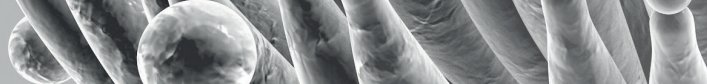

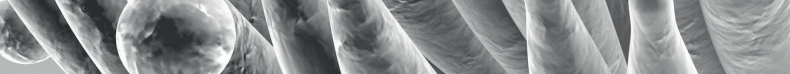

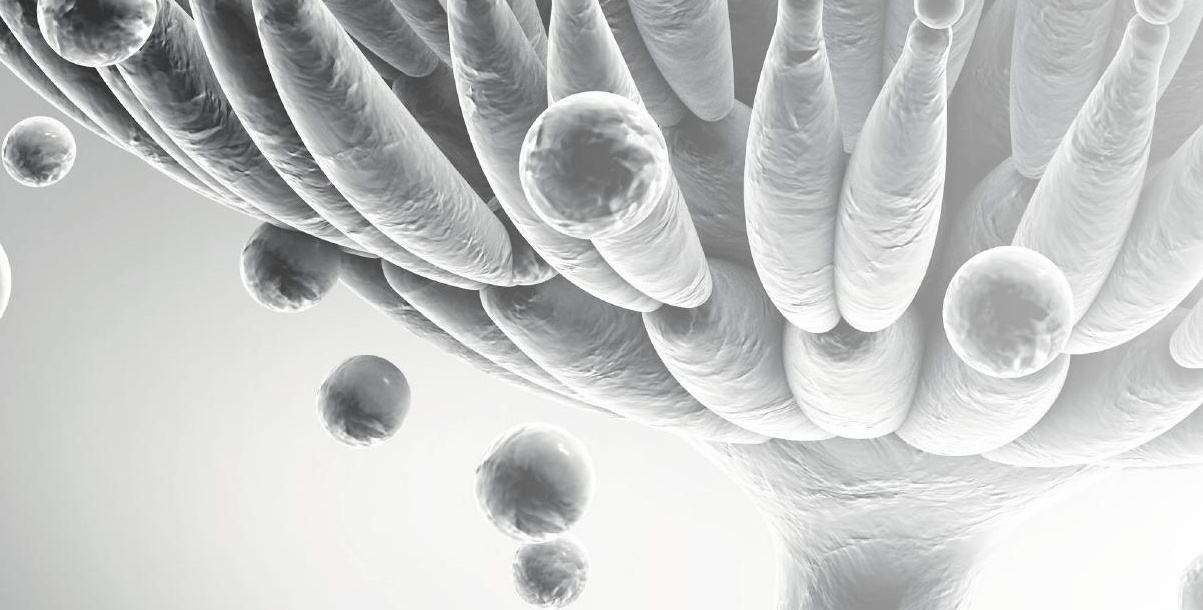

(.

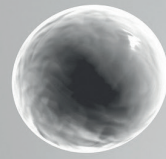

6 
Appendices 


\section{Summary}

Itaconic acid (2-methylidenebutanedioic acid) is an important building block chemical that can be produced from sugars via chemical or biological conversions. Itaconic acid can be processed into a polymer, which can subsequently be used to replace the petroleum-based polyacrylic acids. Itaconic acid is naturally produced by Aspergillus terreus, certain Ustilago and Candida species and Pseudozyma antarctica. Also in mammalian cells itaconic acid is found during macrophage activation.

Aspergillus niger is a filamentous fungus that is the current host of choice for the production of citric acid on an industrial scale, whereas $A$. terreus is the main natural producer of itaconic acid. The biosynthesis of itaconic acid is very similar to the biosynthesis of citric acid in A. niger. Citric acid concentrations of $200 \mathrm{~g} / \mathrm{L}$ are nowadays obtainable with A. niger. Theoretically, this allows itaconic acid concentrations of over $135 \mathrm{~g} / \mathrm{L}$ to be obtained with A. niger, which is higher that the concentrations currently obtained with $A$. terreus $(80 \mathrm{~g} / \mathrm{L})$. However, in A. niger, cis-aconitate decarboxylase (CadA), the key enzyme for itaconic acid biosynthesis, is missing.

Expression of $A$. terreus CadA in $A$. niger results in the production of a low itaconic acid concentration $(0.05 \mathrm{~g} / \mathrm{L})$. The itaconic acid concentration was increased by the expression of a putative mitochondrial transporter (MttA) and a putative plasmamembrane transporter (MfSA). Expression of the MttA transporter in an itaconic acid producing A. niger strain resulted in a twenty-fold increase in itaconic acid secretion. Expression of the $A$. terreus itaconic acid cluster consisting of the cadA gene, the $m t t A$ gene and the $m f s A$ gene resulted in $A$. niger strains that produce over twenty five-fold higher levels of itaconic acid and show a twenty-fold increase in yield compared to a strain expressing only CadA.

To further increase itaconic acid production a modified 6-phosphofructo-1-kinase, $p f k A$, was expressed in a citrate producing $A$. niger strain in combination with cis-aconitate decarboxylase, cadA, from $A$. terreus. The combined expression of pfkA and cadA resulted in increased citrate levels, but did not show increased itaconic acid levels. The combined expression of pfkA with the itaconic acid biosynthetic cluster resulted in significantly increased itaconic acid production at earlier time points. Also the itaconic acid productivity increased significantly. The maximum itaconic acid productivity that was reached under these conditions equaled $0.15 \mathrm{~g} / \mathrm{L} / \mathrm{h}$, which is only a factor 17 lower than the $2.5 \mathrm{~g} / \mathrm{L} / \mathrm{h}$ that, according to the US Department of Energy, should be achieved to have an economically feasible production process.

With the requirement of an increasing number of different genes that needed to be integrated in the genome of $A$. niger, there appeared a need for an efficient flexible pathway 
transfer system. The developed Funbrick system enables easy and quick construction of expression vectors for integration of pathways and the construction of localization vectors. A major advantage of this system is the ability to freely alter the so- called Funbrick after its construction. The Funbrick system was successfully applied by integration of the itaconic acid biosynthesis gene cluster of $A$. terreus in the genome of $A$. niger. The highest itaconic acid concentration obtained equaled $169 \mathrm{mg} / \mathrm{L}$. In addition, the Funbrick system was used to localize the itaconic acid biosynthesis proteins.

In order to further increase the efficiency of pathway transfer in A. niger and other filamentous fungi, we developed a non-laborious way for the rapid selection of transformants resulting from integration at a defined locus in the genome. In A. niger, homologous or targeted integration is relatively rare. The $A$. niger genome contains a spore color formation gene fwnA, which was investigated as a selective marker for homologous integration. Transformants resulting from homologous recombination at the fwnA locus can be rapidly distinguished on the basis of their spore color, which is fawn.

Overall, itaconic acid production has been successfully established in A. niger, albeit in low concentrations, by expressing the gene encoding the key enzyme CadA. Expression of the transporters MttA and MfsA from A. terreus in A. niger showed that especially the mitochondrial MttA transporter has a major effect on the production of itaconic acid in the host strain A. niger NW186. Overall, transport processes play a major role in itaconic acid production and the focus thereon has led to large improvements in itaconic acid production by $A$. niger. 


\section{Samenvatting}

Itaconzuur (methyleenbarsteenzuur) is een belangrijke chemische bouwsteen die uit suikers kan worden geproduceerd via chemische of biologische omzettingen. Itaconzuur kan worden verwerkt tot een polymeer, dat vervolgens kan worden gebruikt om polyacrylzuren, die geproduceerd worden op basis van petroleum, te vervangen. Itaconzuur wordt geproduceerd door Aspergillus terreus, bepaalde Ustilago en Candida soorten en Pseudozyma antarctica. Ook in zoogdiercellen is itaconzuur aangetroffen tijdens activatie van macrofagen.

Aspergillus niger is een draadvormige schimmel die momenteel de meest gekozen gastheer is voor de productie van citroenzuur op een industriële schaal, terwijl A. terreus de voornaamste natuurlijke producent van itaconzuur is. De biosynthese van itaconzuur lijkt erg op de biosynthese van citroenzuur in A. niger. Citroenzuurconcentraties van 200 $\mathrm{g} / \mathrm{L}$ zijn tegenwoordig haalbaar met $A$. niger. Theoretisch zou dit itaconzuurconcentraties van meer dan $135 \mathrm{~g} / \mathrm{L}$ in $A$. niger kunnen opleveren, wat hoger is dan de concentraties die momenteel met $A$. terreus behaald worden $(80 \mathrm{~g} / \mathrm{L})$. Echter, A. niger heeft geen cisaconitaat decarboxylase (CadA), het enzym dat nodig is voor de biosynthese van itaconzuur.

Expressie van A. terreus CadA in A. niger resulteert in een lage itaconzuurproductie $(0,05$ $\mathrm{g} / \mathrm{L}$ ). De itaconzuurconcentratie werd verhoogd door een vermoedelijke mitochondriale transporter (MttA) en een vermoedelijke plasmamembraantransporter (MfsA) tot expressie te brengen. Expressie van de MttA-transporter in een itaconzuur producerende A. niger stam resulteerde in een verhoging van itaconzuursecretie met een factor 20. Expressie van het $A$. terreus itaconzuurcluster bestaande uit het cadA-gen, het $m t t A$-gen en het $m f s A$ gen leidde tot $A$. niger stammen die meer dan 25 keer zoveel itaconzuur produceerden en een 20 keer hoger rendement behaalden in vergelijking met een stam die alleen CadA tot expressie bracht.

Om de itaconzuurproductie nog verder te verhogen werd een gemodificeerde 6-fosfofructo1-kinase, $p f k A$, tot expressie gebracht in een citraat producerende $A$. niger stam in combinatie met cis-aconitaat decarboxylase, cadA, van A. terreus. De gecombineerde expressie van $p f k A$ en cadA resulteerde in verhoogde citraatniveaus, maar leidde niet tot een verhoging van de itaconzuurproductie. De gecombineerde expressie van $p f k A$ met het itaconzuurbiosynthesecluster resulteerde in een significant verhoogde itaconzuurproductie tijdens de vroege meetpunten. Ook werd de itaconzuurproductiviteit significant verhoogd. De maximale itaconzuurproductiviteit die werd behaald onder deze omstandigheden was 0,15 $\mathrm{g} / \mathrm{L} / \mathrm{h}$, wat slechts een factor 17 lager is dan de $2,5 \mathrm{~g} / \mathrm{L} / \mathrm{h}$ die volgens het US Department of Energy zou moeten worden behaald om een economisch rendabel productieproces te verkrijgen. 
Doordat er een steeds groter aantal verschillende genen in het genoom van A. niger moest worden geïntegreerd, bleek er een behoefte voor een flexibel systeem te ontstaan om metabole routes efficiënt over te zetten. Het ontwikkelde Funbricksysteem maakt een gemakkelijke en snelle constructie van expressievectoren voor de integratie van metabole routes en de constructie van lokalisatievectoren mogelijk. Een groot voordeel van dit systeem is de mogelijkheid om de zogenoemde Funbrick na de constructie naar wens te kunnen veranderen. Het Funbricksysteem is succesvol toegepast bij de integratie van het itaconzuur-biosynthesecluster van $A$. terreus in het genoom van $A$. niger. De hoogste behaalde itaconzuurconcentratie was 169 mg/L. Het Funbricksysteem is verder nog gebruikt om de itaconzuureiwitten en transporters te lokaliseren.

Om de overzetting van metabole routes naar $A$. niger en andere draadvormige schimmels nog efficiënter te maken hebben we een methode ontwikkeld om transformanten, die resulteren uit de integratie op een vastgestelde plaats in het genoom, eenvoudig en snel te selecteren. In A. niger is homologe of gerichte integratie relatief zeldzaam. Het $A$. niger genoom bevat een gen ( $w n A$ ) dat codeert voor de vorming van de sporekleur. Dit gen is onderzocht als selectieve marker voor homologe integratie. Transformanten die homologe recombinatie op het fwnA-gen hebben ondergaan kunnen snel worden onderscheiden op basis van hun sporekleur, die bruin is.

Concluderend, itaconzuurproductie is, weliswaar in lage concentraties, succesvol tot stand gebracht in A. niger door het gen coderende voor het belangrijkste enzym CadA tot expressie te brengen. Expressie van de transporters MttA en MfsA uit A. terreus in A. niger heeft laten zien dat vooral de mitochondriale MttA-transporter een groot effect heeft op de productie van itaconzuur in de A. niger stam NW186. Transportprocessen blijken een grote rol te spelen in itaconzuurproductie en de focus hierop heeft geleid tot grote verbeteringen in itaconzuurproductie in $A$. niger. 


\section{Acknowledgements}

Af! Na een weg vol hobbels ligt hier dan het resultaat. Deze route ben ik begonnen samen met Leo, vol mooie ideeën, discussies en plannen voor de toekomst heeft het helaas niet zo mogen zijn dat we dit project samen tot een mooi einde konden brengen. Ik hoop dat je er trots op zou zijn geweest. Ik ben in ieder geval dankbaar dat ik mijn PhD onder jouw hoede heb mogen starten en ik heb mooie herinneringen aan alle gesprekken die we hadden over wetenschap, het wetenschappelijke wereldje en het leven daarbuiten. Je was een bijzonder mens.

Ik wil mijn promotoren bedanken en in het bijzonder Willem voor alle steun en hulp na het overlijden van Leo. Zonder jouw hulp weet ik niet of ik ooit de eindstreep gehaald zou hebben. Je hebt een aanstekelijk enthousiasme voor wetenschap en je bleef me verbazen met de snelheid waarop ik de hoofdstukken terug kreeg met uitgebreid en nuttig commentaar.

Ruud, het was voor mij logisch dat jij mijn paranimf zou worden. We hebben heel wat tijd naast elkaar in het kantoor en tegenover elkaar in het lab doorgebracht. Jij hebt laten zien wat doorzettingsvermogen is en dat je ondanks alles gewoon kan promoveren.

Marjolein, al jaren zijn we vriendinnen en samen met Cecile hebben we heel wat uren over onze PhD's gekletst. Bedankt voor al jullie steun en afleiding.

I would like to thank everyone who was involved in the BE-BASIC project. I really enjoyed being part of a big consortium and I'm grateful for all the discussions, ideas and support that I received during all the meetings that we had. Special thanks to my BE-BASIC colleagues at SSB, Juanan, Dorett, Jasper and Tom, thanks for all the discussions and support in and around the lab.

I really enjoyed supervising students during courses and during their thesis. Therefore I would like to thank Jueeli, Sanne, Nick, Ioannis, Floor, Wen, Alicia, Gwen, Prarthana, Marjan, Kevin and Patrick and all the other students that I supervised during courses or helped during iGEM for all your enthusiasm and hard work. I hope you enjoyed your time and learned a lot. It was my pleasure to be your supervisor.

Further I would like to thank all the colleagues of SSB and MIB, friends and family. You all contributed in your own way to my thesis.

Rienk, zonder jouw steun, motiverende woorden en hulp zou er nu niet zo'n mooie thesis liggen. Heel erg bedankt daarvoor!

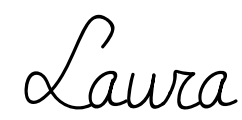




\section{List of publications}

L. van der Straat, J. Tamayo Ramos, T. Schonewille and L. de Graaff. Overexpression of a modified 6-phosphofructo-1-kinase results in an increased itaconic acid productivity in Aspergillus niger. AMB Express 2013,3:57

L. van der Straat, M. Vernooij, M. Lammers, W. Van den Berg, T. Schonewille, J. Cordewener, I. Van der Meer, A. Koops and L. de Graaff. Expression of the Aspergillus terreus itaconic acid biosynthesis cluster in Aspergillus niger. Microb Cell Fact 2014,13:11

L. van der Straat and L. de Graaff. Pathway transfer in filamentous fungi: transporters are the key to success. Bioengineered 2014,5:1-5

E. ten Buren, M. Karrenbelt, M. Lingemann, S. Chordia, Y. Deng, J. Hu, J. Verest, V. Wu, T. Bello Gonzalez, R. van Heck, D. Odoni, T. Schonewille, L. van der Straat, L. de Graaff, and M. van Passel. Toolkit for visualization of the cellular structure and organelles in Aspergillus niger. ACS Synth Biol 2014,3:995-998

L. van der Straat, T Schonewille, JA Tamayo-Ramos and L. de Graaff. Fungal strains with improved citric acid and itaconic acid production. WO 2014/142647 A1, PCT/NL2013/050174, (Filed 14.03.2013, Published 18.09.2014)

L. van der Straat and L. de Graaff. Method for the single step introduction of a plurality of genes in microbial cells. EP2840139A1, (Filed 23.08.2013, Published 25.02.2015)

L. van der Straat and L.H. de Graaff. Optimization strategies for microbial itaconic acid biosynthesis Curr Biotechnol 2017,6:219-226

L. van der Straat, R. Heshof, N. Brouwers, T. Schonewille, R. Van Heck and L. de Graaff. Funbricks, design of versatile expression vectors for synthetic biology of Aspergillus niger. To be submitted

L. van der Straat, I. Papapetridis, N. Brouwers and L. de Graaff. Targeted integration in Aspergillus niger based on a change in spore color. To be submitted 


\section{Overview of completed training activities}

\section{Conferences and meetings}

NVvM meeting

2011

NBV meeting

2011

WCSB meeting, WUR

2012

ECFG11, Marburg

2012

International CeBiTec Research Conference

on Advances in Industrial Biotechnology, Bielefeld

2013

BE-Basic annual meetings

2011-2015

BE-Basic Flagship meetings

\section{Courses}

Seminar on Electron Microscopy, WUR

2012

Advanced course on Microbial Physiology and

Fermentation Technology, TU Delft

2013

\section{General courses}

VLAG introduction week

2011

Competence Assessment, WGS

Teaching and supervising thesis students, WGS

2011

Conversation skills in one-to-one guidance, WGS

2011

Techniques for writing and presenting a scientific paper, WGS

2011

Advanced course Guide to Scientific Artwork, WUR

2012

Workshop Patent search AFSG

\section{Optional courses and activities}

Preparation of PhD proposal

Participation PhD trip USA/Canada

PhD/Postdoc meetings and SSB seminars

Workdiscussions SSB 
The research presented in this thesis was financially supported by a grant in the framework of the BE-BASIC program F01.002 Novel economic and eco-efficient processes for the production of itaconic and fumaric acid

Financial support from the Laboratory of Systems \& Synthetic Biology for printing this thesis is gratefully acknowledged.

Cover design and layout by: Proefschriftenprinten.nl - The Netherlands

Printed by: Print Service Ede: Ede, The Netherlands 


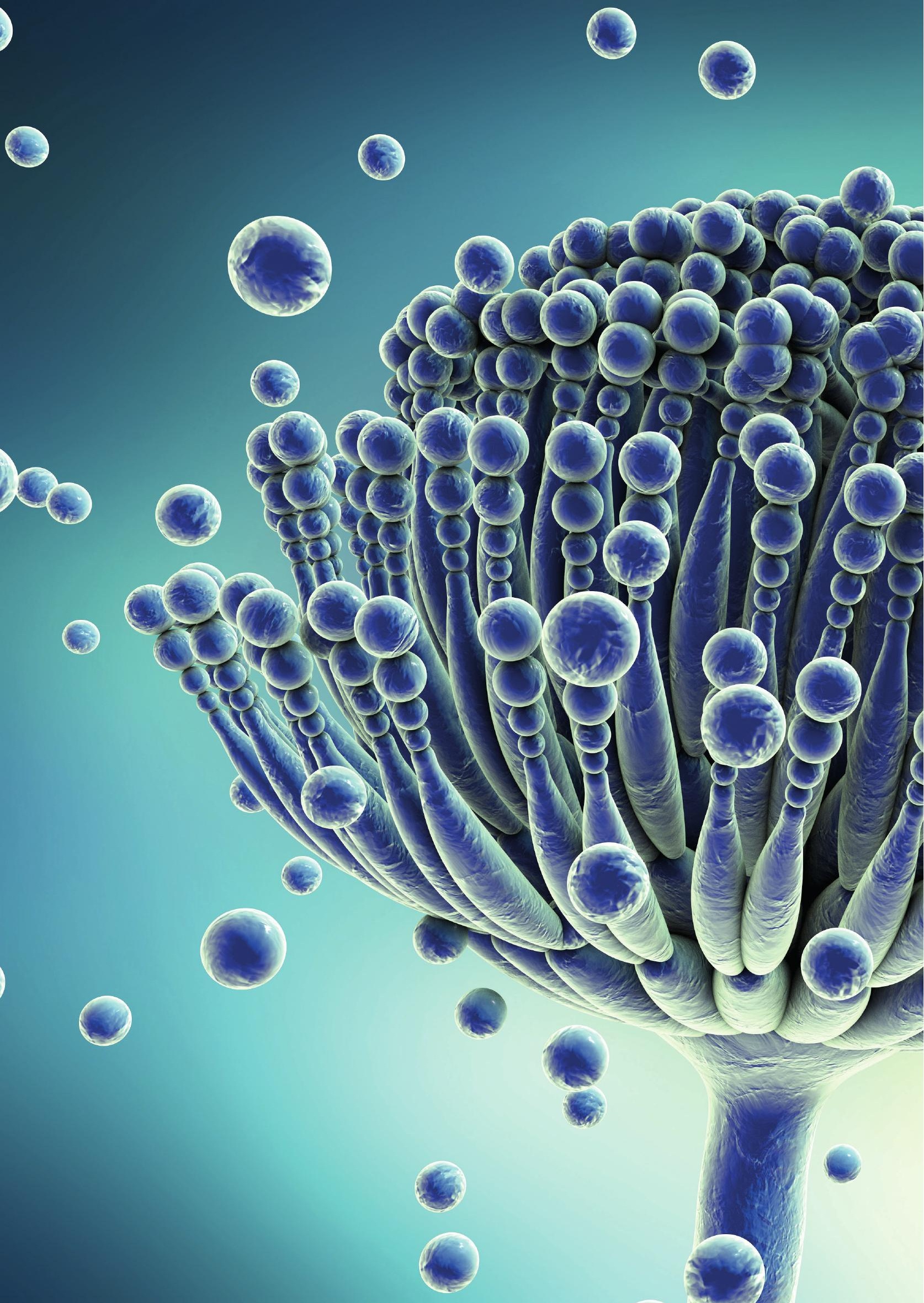

